\title{
IMPLEMENTATION OF LARGE-SYSTEM TRANSFORMATION INITIATIVES IN THE NEW ZEALAND HEALTH SYSTEM
}

\author{
BY
}

\section{KANCHAN M SHARMA}

\author{
A thesis \\ submitted to the Victoria University of Wellington \\ in the fulfilment of the requirements for the degree of \\ Doctor of Philosophy
}

Victoria University of Wellington (2020) 


\section{ABSTRACT}

Health systems worldwide are trying to shift towards a learning system to deliver people-centred, holistic and equitable health care. Large-system transformation (LST) initiatives that capitalise on key features of complex adaptive systems may be more likely to achieve the desired shift.

By LST initiatives, I mean "interventions aimed at co-ordinated, system wide change affecting multiple organisations and care providers, with the goal of significant improvements in the efficiency of health care delivery, the quality of patient care, and population-level patient outcomes" (p 422) [1].

This research had three aims: (1) to identify the key elements that support successful implementation of LST initiatives; (2) to construct a maturity matrix that describes different stages of maturity for each of these elements; and (3) to investigate and report on contextual factors that influence successful implementation of LST initiatives. Collectively, the three aims revealed the programme architecture that underpins efforts to successfully implement LST initiatives in the New Zealand health system.

The realist logic of enquiry, nested within the macro framing of complex adaptive systems, formed the overall methodology for this research. This research used insights from a New Zealand LST initiative (the System Level Measures programme), evidence from literature, and evidence from knowledge of those working in the health system, to analyse and describe this programme architecture.

The research resulted in three key sets of findings.

First, the research found that a set of 10 key elements needs to be present in the New Zealand health system and work in harmony to increase the chances of successful implementation of LST initiatives. These are: (i) an alliancing way of working; (ii) a commitment to Te Tiriti o Waitangi; (iii) an understanding of equity; (iv) clinical leadership and involvement; (v) involved people, whanau and community; (vi) intelligent commissioning; (vii) continuous improvement; (viii) an integrated health information; (ix) analytic capability; and (x) dedicated resources and time.

Second, a self-assessment maturity matrix for the key elements was developed with New Zealand health system leaders to provide a practical tool for them and informal trust-based networks (such as Alliances) to improve their understanding of the different stages of maturity for the key elements, to assess their readiness for change, and to develop capacity and capability needed for system transformation. 
Third, a realist logic of enquiry was used to investigate how the key elements work in different contexts to influence the successful implementation of LST initiatives. At a local level, (i) the history of working together and quality of relationships, (ii) distributed leadership from commissioners of health services, and (iii) the maturity of informal trust-based networks, such as Alliances, emerged as key contextual factors that influenced successful implementation of these initiatives. The key mechanism of trust was triggered with a positive history of working together, which built strong relationships and facilitated a distributed leadership style among health system agents through informal networks. The high-trust environment built and nurtured over time strengthened relationships among health system agents, which then provided the foundation for health system transformation.

At a national level, the distributed health system leadership, the application of 'new power' approach to design and implementation of LST initiatives, and the system accountability environment emerged as key contextual factors. The existing accountability framework, which solely focussed on financial performance of District Health Boards and outputs, suffocated the notion of a learning system as health system leaders placed more effort on achieving targets and outputs rather than on continuous improvement. A culture of continuous improvement supported the notion of a learning system; it encouraged iterative learning using methods such as plan-do-study-act cycles and fostered innovation. Use of 'new power' values such as collaborative policy design and implementation harnessed the intrinsic motivation of health system agents and built trust between policy makers and health service providers, which lead to sustained collective engagement with transformation efforts. A collective engagement to achieve a shared vision built strong and resilient health system leadership.

The research concluded that transformation of health systems depended on senior system leaders' understanding of the programme architecture that underpins efforts to successfully implement LST initiatives. 
This thesis is dedicated to my two beautiful daughters:

Aina and Anushka.

May this journey of mine to reach the highest accolade of academic achievement inspire you to achieve your own academic potential. Thank you, my darlings, for your support. 


\section{ACKNOWLEDGEMENTS}

This research has been an extraordinary journey that could not have been successfully completed without the support and guidance of many people.

First and foremost, I would like to thank all the research participants for taking the time out of their busy schedules to share their experience and knowledge. I was overwhelmed with the support from participants, both in New Zealand and overseas. I would like to especially acknowledge the workshop participants and their employers, who gave this research two days of their valuable time. I have tried my best to represent contributions of all participants earnestly and accurately.

I would like to thank Dr Peter Jones, who has led the System Level Measures programme with me since 2015. I am grateful that Peter granted me consent so I could duly acknowledge him and his contribution. Peter assisted me with data collection by facilitating workshops and Alliance leadership team meetings, provided editorial advice on my thesis, and offered continuous support and encouragement from the beginning to the end of this journey. I am also grateful to Dr Jones for providing financial assistance by paying for transcription costs from his research fund at the University of Auckland. Thank you, Dr Jones for your support, for sharing your knowledge and for giving so much of your time to this research.

I am very grateful to my supervisors: Professor Jackie Cumming and Dr Lesley Middleton. They supported, encouraged and guided me throughout this journey. I really appreciated your constructive feedback along the way to produce a thesis that I am extremely proud of. It has been a privilege for me to work with Prof Cumming, who is highly regarded in the New Zealand health research community. I am very thankful to Dr Middleton, who helped me trouble-shoot my concerns and for always having an article or a book chapter to clear my 'writer's block'.

I would like to thank Kristie Saumure, senior librarian at the Ministry of Health. Kristie helped me with my information searches and was always on the lookout for literature that she thought would help me with my research. Thank you, Kristie, also for finding answers to my endless endnote referencing questions.

I would like to acknowledge the efforts of Andy Inder, who was my manager at the Ministry of Health during the early stages of this journey. I enjoyed our conversations about the challenges and opportunities in the health system, which formed the ideas that led to the early conceptual 
design of the research. Andy also helped me identify research participants for the workshops and the interviews. Thank you, Andy, for your support and encouragement.

My thanks to Jude Ryks, for transcribing my audio recordings promptly and with such high quality. I am grateful to the Victoria University of Wellington for the research grant to assist with my data collection. I would like to thank my father-in-law, Reggie Ponniah, for proofreading and Janice Tomlin for formatting the final version of my thesis.

Last, but not least, I would like to thank my husband, Raaj, for his unwavering support from the time I decided to embark on this challenging journey. Thank you for sharing the highs and the lows of this journey and always reassuring my confidence. I am constantly amazed by the confidence you have in my abilities. I could not have achieved this milestone in my life without you and I am so grateful that you are standing beside me to celebrate this achievement. 


\section{CONTENTS}

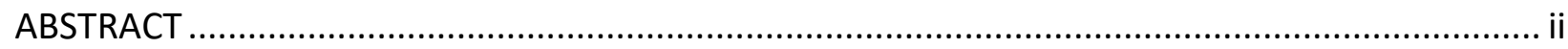

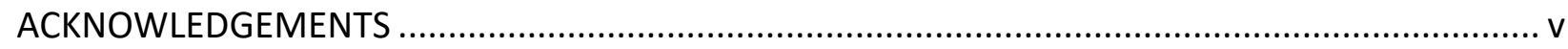

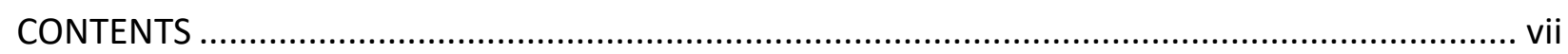

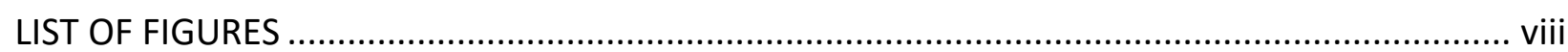

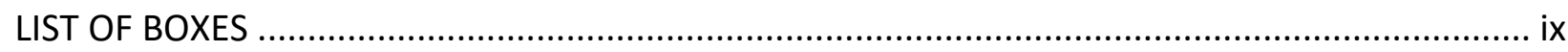

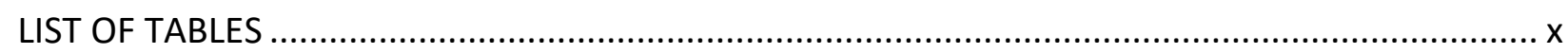

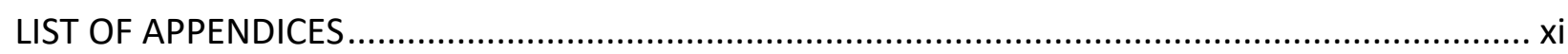

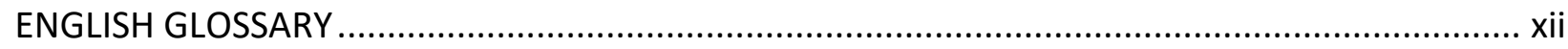

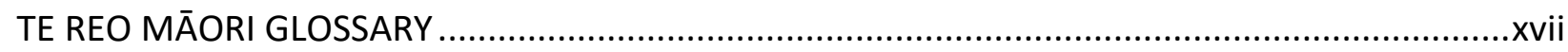

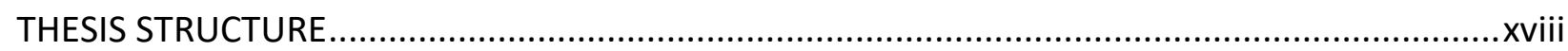

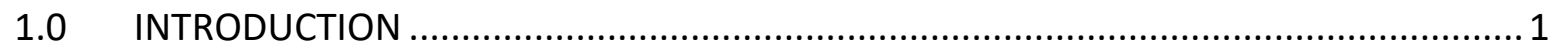

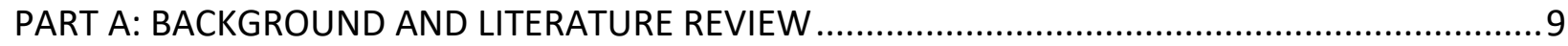

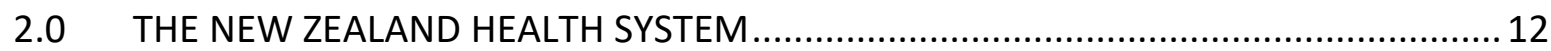

3.0 THE NEW ZEALAND SYSTEM LEVEL MEASURES PROGRAMME................................. 37

4.0 ACADEMIC CONTEXT FOR THE PROPOSED RESEARCH ........................................ 49

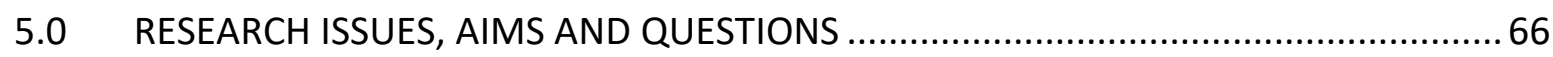

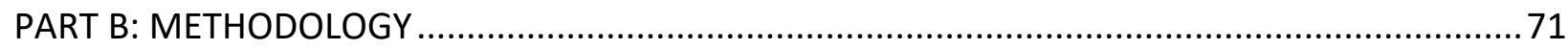

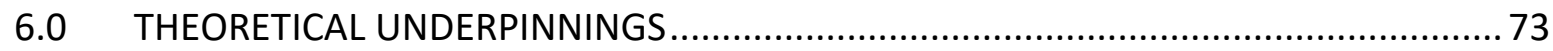

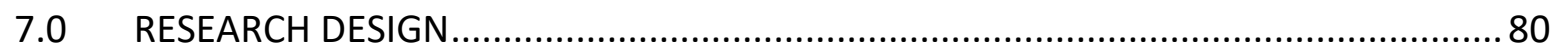

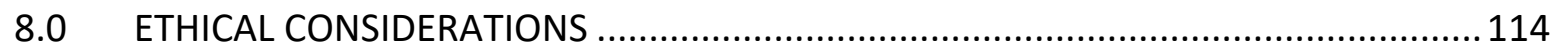

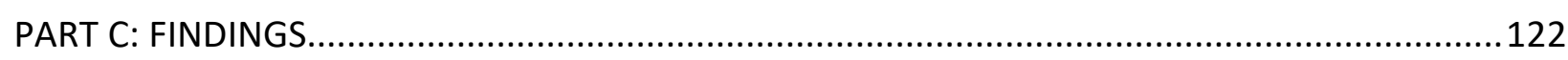

9.0 KEY ELEMENTS TO SUPPORT SUCCESSFUL IMPLEMENTATION OF LST INITIATIVES 125

10.0 THE SELF-ASSESSMENT MATURITY MATRIX

11.0 CONTEXTS AND MECHANISMS THAT INFLUENCE SUCCESSFUL IMPLEMENTATION OF

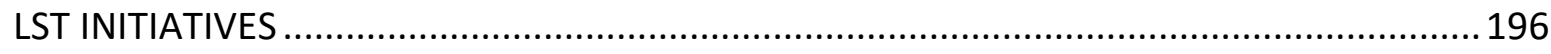

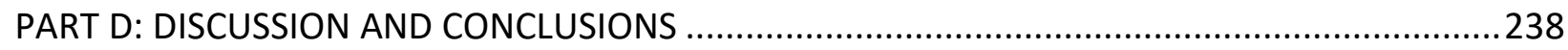

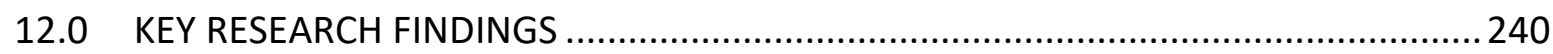

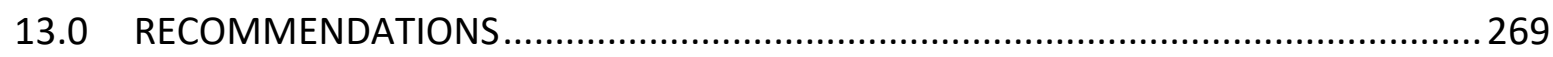

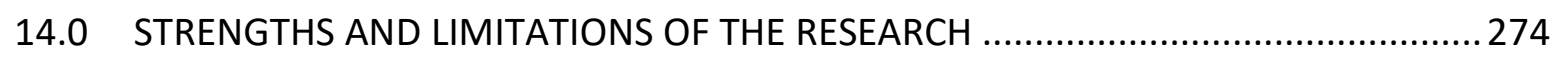

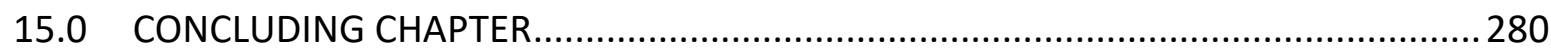

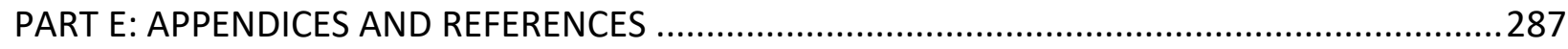

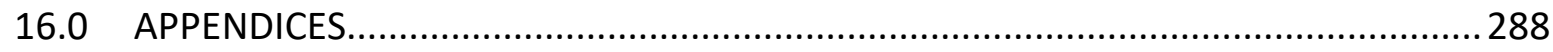

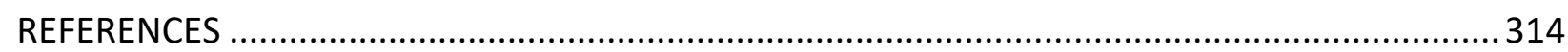

Kanchan M Sharma vii 


\section{LIST OF FIGURES}

Figure 1.1: Successful implementation of LST initiatives ....................................................... 4

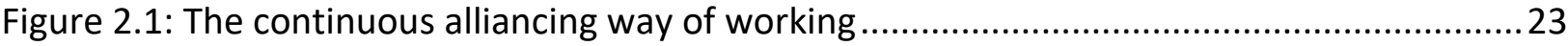

Figure 3.1: Improvement plan development process ............................................................. 41

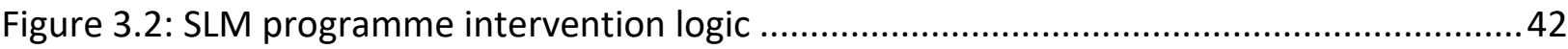

Figure 4.1: Simple representation of complex adaptive systems, adapted from The Health Foundation [128]

Figure 4.2: Illustration of 'Old and New Power', adapted from Heimans and Timms [128] and

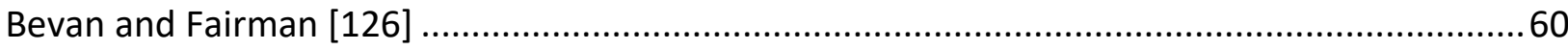

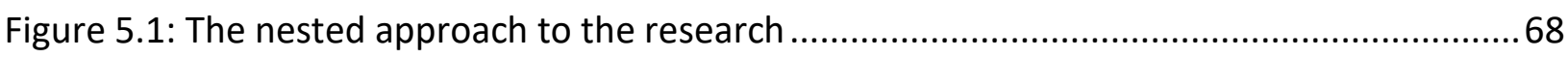

Figure 7.1: Realist research design, adapted from Marchal [182] ........................................... 81

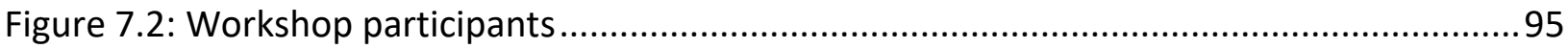

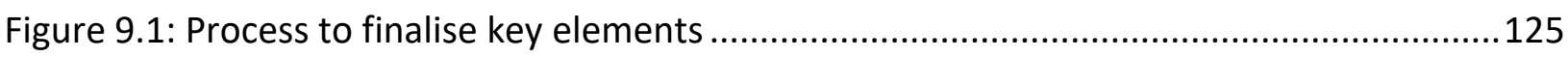

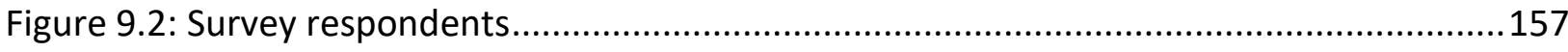

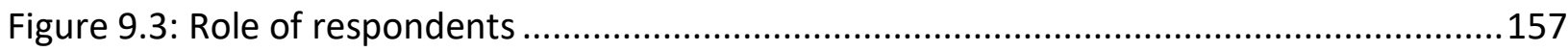

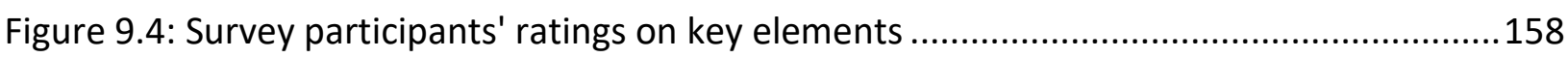

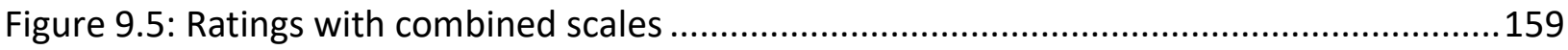

Figure 10.1: Iterative process for refining the maturity matrix ..........................................161

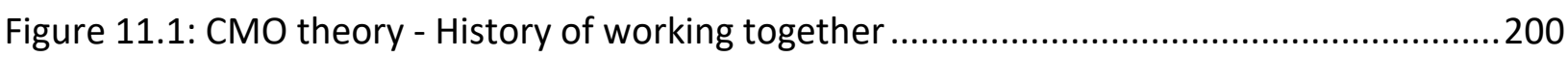

Figure 11.2: CMO theory - Distributed leadership from DHB leaders ....................................204

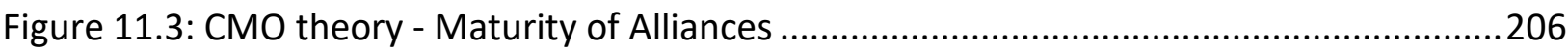

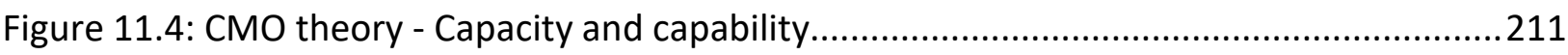

Figure 11.5: CMO theory - Continuous improvement culture ...............................................213

Figure 11.6: CMO theory - 'New power' approach to design and implementation of LST initiatives

Figure 11.7: CMO theories - Political environment............................................................218

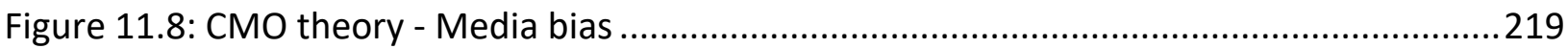

Figure 11.9: CMO theory - The MoH leadership ................................................................221

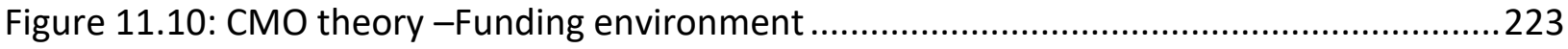

Figure 11.11: CMO theory - System accountability environment..........................................226

Figure 11.12: CMO theory - Training of health care professionals .......................................227

Figure 11.13: CMO interplay showing the ripple effect of the trust mechanism over time, adapted

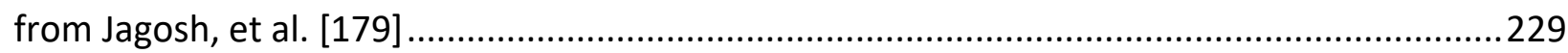

Kanchan M Sharma viii 
Figure 13.1: New proposed national accountability framework

Figure 15.1: Updated SLM programme intervention logic with summary of realist mechanisms triggered by local and national contexts, adapted from Middleton, et al. [225]

\section{LIST OF BOXES}

Box 2.1: Alliancing principles [42] 22

Box 2.2: Proposed shared system values [23] 34

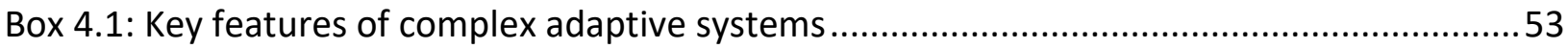

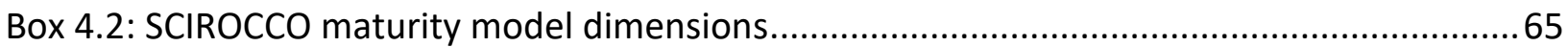

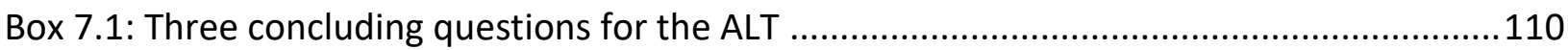




\section{LIST OF TABLES}

Table 2.1: Doctrines of 'new public management' reproduced from Hood [79] ........................25

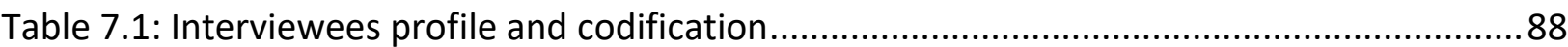

Table 7.2: Topic guide for realist interviews with research participants ...................................91

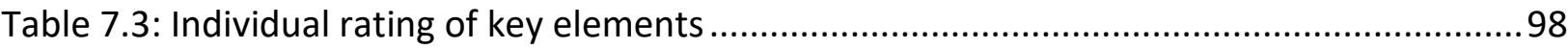

Table 7.4: Finalising key element definitions and outcome descriptors ................................102

Table 7.5: Maturity matrix outline adapted from Kirk, et al. [155] .......................................103

Table 8.1: Map of research questions, theory and methods of data collection and analysis ....121

Table 9.1: Initial list of key elements gleaned from my insights.

Table 9.2: Primary and secondary drivers for successful implementation of LST initiatives [30]

Table 9.3: Key attributes of high performing health care systems [122]

Table 9.4: Organisational context and capabilities for integrating care [3]

Table 9.5: Revised list of key elements and descriptions used for interviews and workshops..135

Table 9.6: Interview participants' ratings on revised list of key elements (health)....... 137

Table 9.7: Interview participants' ratings on revised list of key elements (non-health) 137

Table 9.8: Additional elements identified by interview participants. 148

Table 9.9: Key elements before and after the interviews. 149

Table 9.10: Workshop participant ratings on the revised list of key elements 150

Table 9.11: Additional elements identified by workshop participants 153

Table 9.12: Workshop participants' consensus on the key elements 154

Table 9.13: Consolidation of key elements and descriptions following interviews and workshops 156

Table 9.14: Survey participants ratings on key elements 158

Table 9.15: Consolidation of key elements 160

Table 10.1: First version of the maturity matrix 173

Table 10.2: Outline of the maturity matrix following first testing 174

Table 10.3: Maturity Matrix for New Zealand Alliances - a self-assessment tool to support largescale change in health system (final version) 195

Table 11.1: Consolidated CMO theories for successful implementation of LST initiatives in the New Zealand health system 235

Table 12.1: Key elements from this research mapped to similar elements from literature ......242

Table 12.2: Key elements mapped to Human Learning Systems Dimensions 261 


\section{LIST OF APPENDICES}

Appendix 1: Structure of the New Zealand health system [66] ..........................................28

Appendix 2: Literature review summary of LST initiatives ................................................289

Appendix 3: Research information sheet for participants ....................................................293

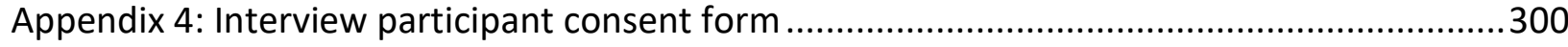

Appendix 5: Chief Executive participant information sheet and consent form ........................301

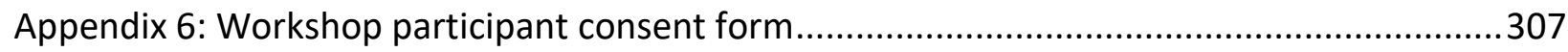

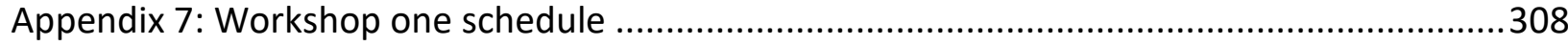

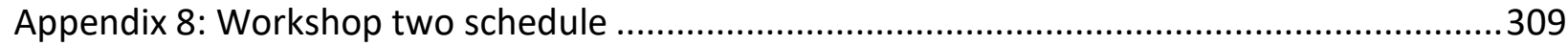

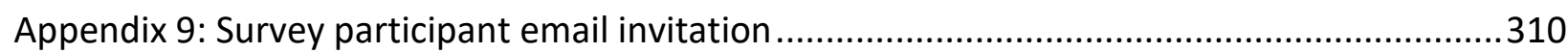

Appendix 10: Alliance participant consent form....................................................................

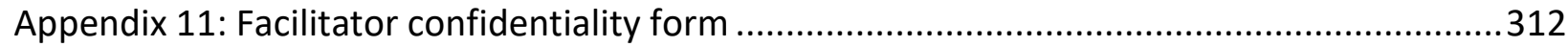

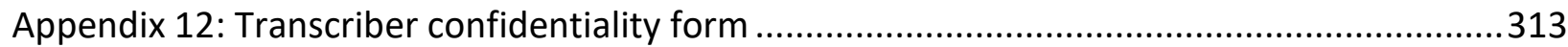




\section{ENGLISH GLOSSARY}

\begin{tabular}{|c|c|}
\hline Term & Definition \\
\hline $\begin{array}{l}\text { Alliance or } \\
\text { district Alliance }\end{array}$ & $\begin{array}{l}\text { An Alliance is an informal trust-based network that aims to bring all agents } \\
\text { in the local health system together with the aim of transforming services, } \\
\text { so that services work best for the patient and best for the system. } \\
\text { Alliancing is a collaborative way of working, reliant on trusted } \\
\text { relationships and supported by enablers critical to continuous } \\
\text { improvement. Successful Alliances serve the interests of the community } \\
\text { while preserving the autonomy of multiple organisations and services } \\
\text { present in a complex system. } \\
\text { New Zealand District Health Boards are required to form an Alliance with } \\
\text { the Primary Health Organisations providing health services to the } \\
\text { population of their district [2]. }\end{array}$ \\
\hline $\begin{array}{l}\text { Alliance } \\
\text { Leadership Team } \\
\text { (ALT) }\end{array}$ & $\begin{array}{l}\text { These leadership teams have varied membership depending on the make- } \\
\text { up of the Alliances. Some Alliances have a broad membership that include } \\
\text { senior District Health Board and Primary Health Organisation managers } \\
\text { and clinicians, consumer advocates, and representatives from community } \\
\text { health services such as pharmacy, Māori and Pacific providers and district } \\
\text { nursing. Other Alliances only have senior District Health Board and } \\
\text { Primary Health Organisation managers and clinicians. }\end{array}$ \\
\hline Capability & $\begin{array}{l}\text { A person or organisation's ability and capacity to perform a set of tasks. } \\
\text { This includes skills and knowledge of individuals and the ability of an } \\
\text { organisation to harness the collective knowledge of individuals as well as } \\
\text { having the necessary organisational structures, processes, culture and } \\
\text { tools to perform tasks successfully [3]. }\end{array}$ \\
\hline Capacity & $\begin{array}{l}\text { The opportunities and resources available to a person or an organisation } \\
\text { to change. Individual capacity refers to attitudes, beliefs and intentions to } \\
\text { change. Organisational capacity refers to resources and culture needed to } \\
\text { implement and sustain change [3]. }\end{array}$ \\
\hline $\begin{array}{l}\text { Context(s) or } \\
\text { contextual } \\
\text { factors }\end{array}$ & $\begin{array}{l}\text { These are organisational, social, cultural and political conditions that exist } \\
\text { in the health system and in the process of implementing LST initiatives, }\end{array}$ \\
\hline
\end{tabular}




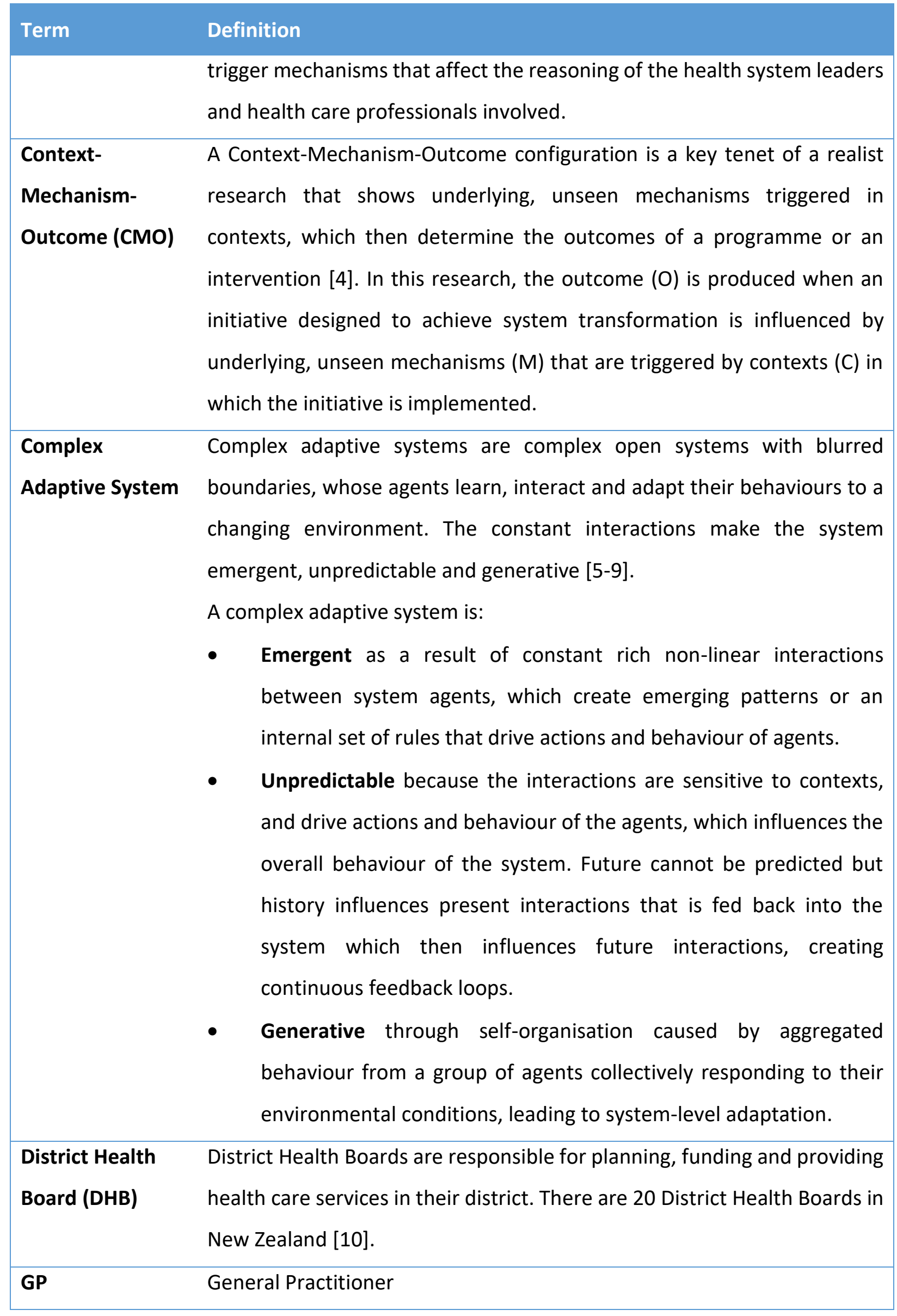




\begin{tabular}{|c|c|}
\hline Term & \\
\hline system & $\begin{array}{l}\text { "The health system focuses on the health and well-being of the person" } \\
\text { (p 1) [5]. } \\
\text { In this thesis, health system refers to the health and disability system that } \\
\text { is responsible for planning, funding, delivering and managing health } \\
\text { services to its population. }\end{array}$ \\
\hline $\begin{array}{l}\text { Large-system } \\
\text { transformation } \\
\text { (LST) initiatives }\end{array}$ & $\begin{array}{l}\text { These are "interventions aimed at co-ordinated, system wide change } \\
\text { affecting multiple organisations and care providers, with the goal of } \\
\text { significant improvements in the efficiency of health care delivery, the } \\
\text { quality of patient care, and population-level patient outcomes" (p 422)[1]. } \\
\text { Large-system transformation initiatives are designed to achieve system } \\
\text { transformation, or large-scale change. Here, these are labelled large- } \\
\text { system transformation initiatives, or LST initiatives. }\end{array}$ \\
\hline Learning system & $\begin{array}{l}\text { In a learning system, agents are attuned to a system's features, apply } \\
\text { systems thinking and use feedback loops to nudge the behaviour of the } \\
\text { system out of equilibrium and thereby build momentum for change [11]. }\end{array}$ \\
\hline Mechanisms & $\begin{array}{l}\text { Mechanisms are the underlying, unseen reasoning of health system } \\
\text { leaders and heath care professionals prompted by the opportunities and } \\
\text { resources available to them that determine their willingness and ability to } \\
\text { support, lead and or be involved in the implementation of LST initiatives. } \\
\text { These mechanisms influence the actions and behaviours of these leaders } \\
\text { and health care professionals in the health system, which then influence } \\
\text { the implementation of LST initiatives. }\end{array}$ \\
\hline $\begin{array}{l}\text { Ministry of } \\
\text { Health }(\mathrm{MoH})\end{array}$ & $\begin{array}{l}\text { The New Zealand central agency responsible for leadership of the health } \\
\text { system. The MoH shapes national policy environment that includes } \\
\text { briefing the incoming Minister of Health, ongoing policy advice to the } \\
\text { Minister of Health, implementing priorities and policies of the } \\
\text { government, and demonstrating the value and confidence of system to } \\
\text { the government and the public through appropriate accountability } \\
\text { instruments [12]. }\end{array}$ \\
\hline ‘New Power’ & $\begin{array}{l}\text { 'New power' comes from relationships and connections and uses people's } \\
\text { intrinsic motivation to influence and call for action to achieve a shared }\end{array}$ \\
\hline
\end{tabular}




\begin{tabular}{|c|c|}
\hline Term & Definition \\
\hline & $\begin{array}{l}\text { purpose or vision. While the 'old power' focuses on accountability, } \\
\text { targets, sanctions, and command and control, the 'new power' leans } \\
\text { towards shared common purpose, collective action, collaboration, } \\
\text { partnership, high-trust relationships, and harnessing energy [13]. }\end{array}$ \\
\hline NPM & New Public Management \\
\hline $\begin{array}{l}\text { Operational } \\
\text { Policy } \\
\text { Framework (OPF) }\end{array}$ & $\begin{array}{l}\text { The Operational Policy Framework (OPF) contains business rules, policies } \\
\text { and guidelines that outline the operating functions of DHBs. It is endorsed } \\
\text { by the Minister of Health and DHBs are responsible for complying with the } \\
\text { OPF [2]. }\end{array}$ \\
\hline Outcomes & $\begin{array}{l}\text { The outcomes in this research are intermediate outcomes, i.e. the changes } \\
\text { brought about, both intended and unintended, that have a significant } \\
\text { influence on the successful implementation of LST initiatives. These } \\
\text { intermediate outcomes are useful to bridge the gap between the theory } \\
\text { of LST initiatives and the reality of implementing these initiatives in a } \\
\text { complex adaptive system. }\end{array}$ \\
\hline $\begin{array}{l}\text { Plan-Do-Study- } \\
\text { Act (PDSA) }\end{array}$ & $\begin{array}{l}\text { The Plan-Do-Study-Act cycle is a tool used to monitor change in a } \\
\text { continuous improvement cycle by developing a plan for the change (Plan), } \\
\text { carrying out the change (Do), observing and learning from the change } \\
\text { (Study), and making improvements to the change (Act). }\end{array}$ \\
\hline $\begin{array}{l}\text { Primary Health } \\
\text { Organisation } \\
(\mathrm{PHO})\end{array}$ & $\begin{array}{l}\text { Primary Health Organisations are funded by the District Health Boards to } \\
\text { provide essential primary care services through general practices to those } \\
\text { who are enrolled with. There are } 30 \text { Primary Health Organisations in New } \\
\text { Zealand [10]. }\end{array}$ \\
\hline $\begin{array}{l}\text { Programme } \\
\text { architecture }\end{array}$ & $\begin{array}{l}\text { In this research, programme architecture is a term used to describe the } \\
\text { organisational context and capabilities that underpin efforts to } \\
\text { successfully implement large-system transformation initiatives. The term } \\
\text { evolved during the writing of the thesis to collectively describe the many } \\
\text { facets that need to come together to drive change within a programme of } \\
\text { work that has the ambitious aim of large-system transformation. }\end{array}$ \\
\hline $\begin{array}{l}\text { Proportionate } \\
\text { universalism }\end{array}$ & $\begin{array}{l}\text { Is an approach that balances targeted and universal health services } \\
\text { through actions proportionate to needs and levels of disadvantage in a }\end{array}$ \\
\hline
\end{tabular}




\begin{tabular}{|ll|}
\hline Term & Definition \\
& population to address health inequities (different treatments for people \\
& with different needs) [14]. Also known as vertical equity. \\
\hline System Level & The System Level Measures (SLM) programme is an initiative designed to \\
Measures (SLM) & achieve large-system transformation in the New Zealand health system. \\
Programme & The programme was introduced to enhance a collaborative way of \\
& working beyond organisational and professional boundaries, address \\
& health inequities and encourage continuous learning and quality \\
& improvement. The key purpose of the programme is to achieve the Triple \\
& Aim [15]. \\
\hline Iriple Aim & Improved quality, safety and experience of care; improved health and \\
& resources [16]. \\
\hline WHO & World Health Organization \\
\hline
\end{tabular}




\section{TE REO MĀORI GLOSSARY}

\begin{tabular}{|c|c|}
\hline Māori & English \\
\hline Aroha & Love \\
\hline Hapū & Sub-tribe \\
\hline Hauora Māori & Māori health, holistic health and wellbeing \\
\hline He Korowai Oranga & Māori Health Strategy \\
\hline Iwi & Tribe \\
\hline Karakia & Prayer \\
\hline Kaupapa Māori & By Māori, for Māori, with Māori \\
\hline Kaupapa Māori services & $\begin{array}{l}\text { Led, owned and governed by Iwi, pan-tribal, or Māori organisations } \\
\text { that are specifically designed with Māori in mind }\end{array}$ \\
\hline Mana & Prestige, authority, influence, status, spiritual power, charisma \\
\hline Manaakitanga & Reciprocity and support \\
\hline Pākehā & New Zealanders primarily of European descent \\
\hline Rangatira & Chief \\
\hline Rangatiratanga & Authority, ownership, leadership, chiefly authority \\
\hline Taonga & Treasure \\
\hline Te Tiriti o Waitangi & $\begin{array}{l}\text { The Treaty of Waitangi, a treaty signed between the British Crown } \\
\text { and Māori rangatira (chiefs) to live together under a common set } \\
\text { of laws and agreements. }\end{array}$ \\
\hline Whakamaua & Māori Health Action Plan 2020-2025 \\
\hline Whakapapa & Kinship ties \\
\hline Whānau & Family and extended family, who are foundation of Māori society \\
\hline Whānau ora & Healthy Families \\
\hline Whenua & Land \\
\hline Whare Tapa Whā & $\begin{array}{l}\text { A holistic health and wellness model of care that represents the } \\
\text { four cornerstones of Māori health: taha tinana (physical health); } \\
\text { taha wairua (spiritual health); taha whānau (family health); and } \\
\text { taha hinengaro (mental health). }\end{array}$ \\
\hline
\end{tabular}




\section{THESIS STRUCTURE}

This research had three aims: first, to identify the key elements that support successful implementation of LST initiatives; second, to construct a maturity matrix that describes different stages of maturity for each of these elements; and third, to investigate and report on how contextual factors that New Zealand alliances work in influence successful implementation of LST initiatives. Collectively, the three aims described the programme architecture that underpins efforts to successfully implement LST initiatives in the New Zealand health system.

The thesis has five parts.

\section{Part A: Background and Literature Review}

Part A sets the scene for the research. It contains four chapters (Chapters 2, 3, 4 and 5). Chapter 2 describes the New Zealand health system: its structure, funding, performance, the history of health care reforms, and the quality of care delivered to New Zealanders. The chapter also explains the alliancing way of working, which is important as these trust-based networks are used to implement the New Zealand System Level Measures (SLM) programme. Chapter 3 describes the SLM programme, and my influence and insights as the National Programme Manager leading the implementation of the programme. These insights and knowledge provided the foundation for the research and were used to develop initial propositions that are tested and refined in the research. Chapter 4 provides the academic context for the research and positions the proposed research within the health system transformation and other literatures relating to accountability and the use of maturity matrices for quality improvement. Drawing from scholarship on complex adaptive systems, this chapter presents the key ideas that shaped both the SLM programme and the initial theory building stage of this thesis. Chapter 5 discusses key knowledge gaps in the existing literature, the research aims and the three research questions that guided the research. 


\section{Part B: Methodology}

Part B comprises three chapters (Chapters 6, 7 and 8). Chapter 6 discusses the theoretical underpinnings and the rationale for choosing a realist logic of enquiry nested within the macro framing of complex adaptive systems. Chapter 7 outlines the research design that includes qualitative and quantitative methods of data collection: (i) theory gleaning from the SLM programme; (ii) a review of published and grey literature; (iii) interviews; (iv) workshops; (v) field testing of the maturity matrix; and (vi) an online survey. Chapter 8 considers ethical issues for the research that includes discussions on how I managed a conflict of interest arising with my dual role as the SLM programme manager and researcher, research confidentiality, and informed consent.

\section{Part C: Findings}

Part C presents the findings of the research and contains three chapters (Chapters 9, 10 and 11). Chapter 9 presents the findings on the different elements that need to be present to support successful implementation of LST initiatives. The chapter starts with insights from the SLM programme followed by a review of evidence from existing literature to form an initial list of required elements. This list was then tested and refined based on New Zealand conditions and experiences through interviews, workshops and an online survey. Chapter 10 drills down on the key elements to describe how each element works in the form of a self-assessment maturity matrix. The maturity matrix was developed with senior system leaders working in the New Zealand health system and outlines the outcome for each element, indicators to measure progress against the outcome, and a maturity scale that shows expectations at four levels of maturity: beginning, emerging, established and excellence. The maturity matrix was designed to support deliberate and conscious learning for New Zealand health system leaders and Alliances. The chapter presents the findings from testing of the maturity matrix with three Alliance leadership teams and feedback from the MoH Māori Health Strategy and Policy team. Chapter 11 applied realist thinking to report on how the key elements work in different contexts and influence the reasoning and behaviour (mechanisms) of system agents, which then have an impact on successful implementation of LST initiatives. The chapter ends with a summary of context-mechanism-outcome (CMO) theories that influence successful implementation of LST initiatives in the New Zealand health system. 


\section{Part D: Discussion and Conclusions}

Part D consists of four chapters (Chapters 12, 13, 14 and 15). Chapter 12 discusses the key findings of the research. It reports how an alliancing way of working is the key element that needs to be present to increase the chances of success with implementation of LST initiatives. The chapter goes on to outline the key contextual factors that influence these initiatives. It pays attention to a history of working, distributed leadership from DHBs, the maturity of Alliances, system leadership, the system accountability environment, and the 'new power' approach to the design and implementation of LST initiatives. Chapter 13 outlines the key recommendations that emerged from the research and Chapter 14 explores the strengths and limitations of the research. The research concludes with Chapter 15. Along with concluding remarks, the chapter deliberates the future of the SLM programme, the contribution of this research and opportunities for future research.

\section{Part E: Appendices and References}

The last part of the thesis contains documents that support the main body of the thesis. The appendices include participant information sheets, consent forms and workshop schedules. This part also includes reference material used in the thesis. 


\subsection{INTRODUCTION}

Health systems world-wide are struggling to meet changing demands arising from longer lifespans, a growing burden of long-term chronic conditions with ageing populations and persistent health inequities [17]. Health systems, which were designed around health institutions and health care professionals to respond to acute episodic care for particular diseases or conditions, need a fundamental shift to systems that are people-centred, deliver seamless co-ordinated holistic care, and acknowledge and address health inequities. This shift requires system transformation in the ways in which health services are funded, managed, and delivered [17].

\subsection{Challenges for the New Zealand health system}

The New Zealand health system is no different in the challenges it is facing and the shift it is seeking to make to meet current and future demands. According to the Global Burden of Disease Study [18], New Zealanders are living longer; however, life expectancy has increased faster than health expectancy. This means that more New Zealanders are spending more years in poor health. About six percent of New Zealand's population is over the age of 75 years. By 2038, the over 65+ population is projected to double to 1.3 million [19]. Like other high-income countries, New Zealand faces a growing challenge with more people living with chronic long-term conditions, including people living with more than one health condition. Non-communicable diseases such as cancers, cardiovascular disease and mental illness are leading contributors to health loss [18]. Health inequities persist between genders, generations, ethnic and socioeconomic groups. Since 1990, health conditions associated with ageing, such as dementia, have increased [18]. As New Zealanders live longer, demand on health system will increase. Going forward, increasing healthy life expectancy through prevention is an important focus to improve the health and wellbeing of the population [18].

Like others, the New Zealand health system has tried several organisational reforms, 'new public management' ideas, and made use of policy instruments including myriad performance and quality indicators to deliver co-ordinated patient-centred care and address health inequities [20]. New Zealand experience has shown that organisational reforms, the use of 'new public management' ideas that have a continuous focus on financial performance, and incoherent implementation of national policies have not succeeded in developing a substantially more integrated and patient-centred approach to the funding, managing and delivery of health care [20-26]. 
Reviewers of the New Zealand health system have consistently recommended a need to focus on strengthening the culture and capacity for system improvement, integration of health services and structures, and aligning resources to achieve the Triple Aim outcome: improved quality, safety and experience of care; improved health and equity for all populations; and best value for public health system resource [21-26].

\subsection{Health system for the $21^{\text {st }}$ century}

Literature emerged in the late 1990s about the notion of health systems being complex adaptive systems [1]. In 2001, 'Crossing the Quality Chasm', from the Institute of Medicine (IOM), called for a redesign of health systems for the $21^{\text {st }}$ century guided by six key dimensions: i.e. systems that are safe, effective, patient-centred, efficient, timely and equitable [27]. The report endorsed the notion of health systems being complex and acknowledged that transformation of a health system "will require crossing a large chasm between today's system and the possibilities of tomorrow" ( $p 4$ ) and the challenging but important role of leadership in doing so [27].

Since then, there has been world-wide recognition that health systems operate as complex adaptive systems, which are made up of many parts with blurred boundaries and whose agents interact, learn and adapt their behaviours depending on the environment in which they work. These constant interactions create feedback loops that make the system emergent, unpredictable and generative $[5,7,9,28,29]$. The emergent, unpredictable and generative nature of health systems make change or transformation initiatives challenging. While small-scale changes in individual care settings have been successful, rapid and broad scaling-up and spreading of these have remained a challenge [30]. Large-system transformation (LST) initiatives, which use complexity thinking and the 'new power' approach have been theorised as more likely to bring about the paradigm shift in thinking and the transformation needed to meet the demands of current and future health systems [1,6,27,29,31-35].

\subsection{Large-system transformation initiatives}

By LST initiatives, I mean "interventions aimed at co-ordinated, system wide change affecting multiple organisations and care providers, with the goal of significant improvements in the efficiency of health care delivery, the quality of patient care, and population-level patient outcomes" (p 422) [1]. 
The three key aspects of LST initiatives are that (i) these are broad and widespread across geographical boundaries, multiple organisations or across professional groupings; (ii) these challenge current way of thinking and seek paradigm shifts in mindsets, processes and relationships; and (iii) these affect people and require co-ordination across multiple systems nested within a macro system [32]. LST initiatives are not about organisational changes or incremental improvements of current processes [1,32]. LST initiatives are complex interventions implemented in a complex adaptive system.

\subsection{Successful implementation of large-system transformation initiatives}

The literature tells us that successful implementation of LST initiatives requires integrated changes in structures, processes and behaviour patterns $[1,32,36]$. It involves creating positive conditions for change to support a work environment that harnesses relationships, and increases the capacity and capability of those working in the system [1]. The focus is less about meeting targets and is more about iterative planning and practice cycles to shift system behaviour [1]. Senior leaders of the system, especially from organisations with largest amount of resources, have to let go of control and the traditional 'old power' approach. The 'old power' approach is leader-driven with transactional dynamics between funders and providers of services and uses targets and sanctions to achieve outputs [13]. Instead successful implementation of LST initiatives in a complex adaptive system is thought to require a 'new power' approach [35]. 'New power' comes from relationships and connections, and uses people's intrinsic motivation to influence and call for action to achieve a shared purpose or vision [13,35].

In the short-term, it is almost impossible to show hard evidence of attribution for successful implementation of LST initiatives. Short-term success requires health system agents to muddle through many complex and unexpected factors, patterns, and relationships that need to be carefully crafted, nurtured and developed [37].

Over time, changes in attitudes, beliefs, and behaviours become natural and long-term outcomes of system transformation are achieved [32]. The evidence base for successful implementation of LST initiatives is therefore not about meeting targets or determining 'what works'. Instead, rich descriptions about 'what works, for whom, under what circumstances, and why' are needed to understand the success of these initiatives [1]. In this research, this evidence base is referred to as the programme architecture that underpins efforts to successfully implement LST initiatives. This programme architecture is useful to bridge the gap between the theory of LST initiatives and the reality of implementing these initiatives in a complex adaptive system. 
Figure 1.1 shows the continuum for successful implementation of LST initiatives and shows the focus and value add of this research along that continuum.
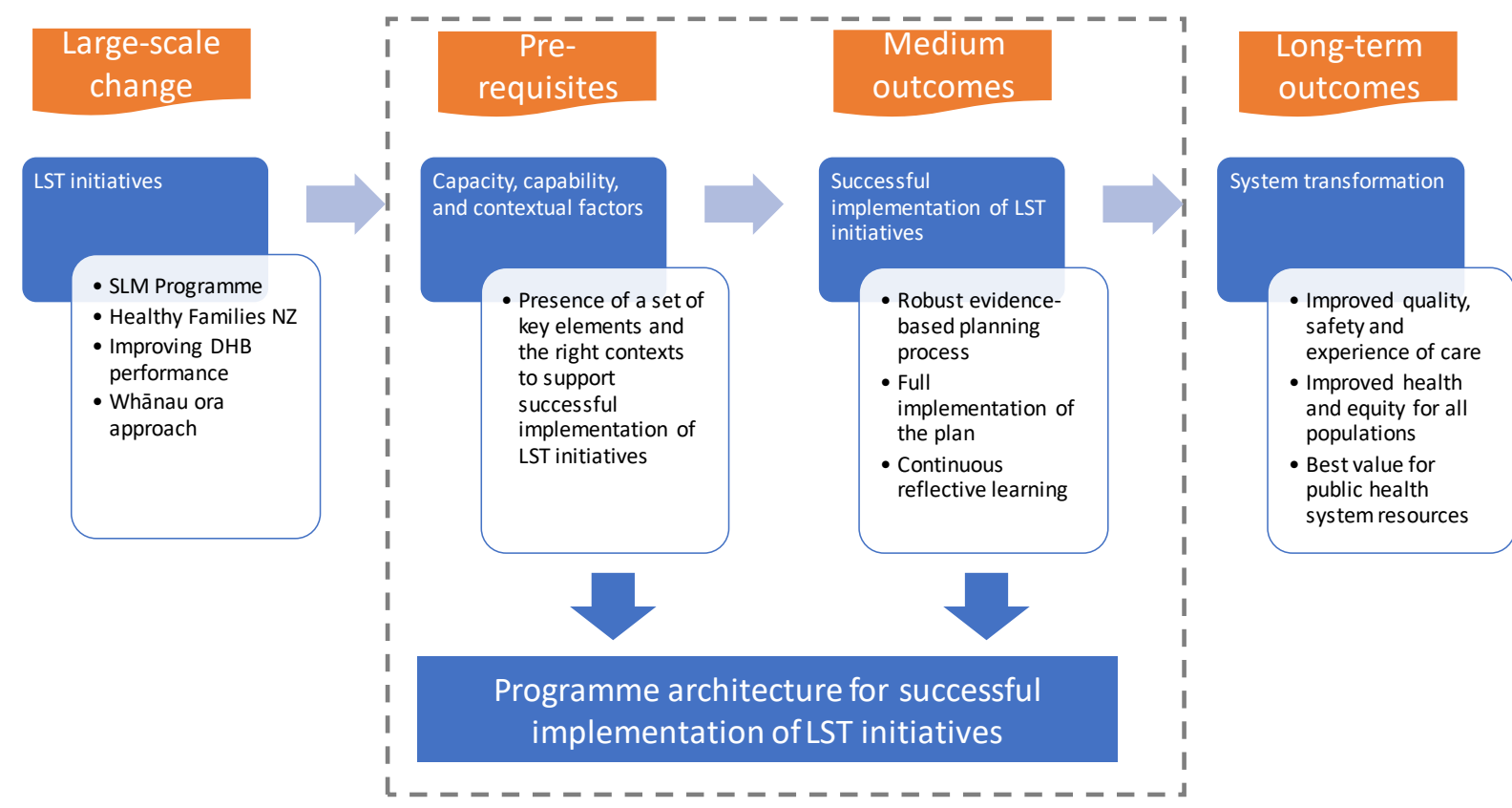

Figure 1.1: Successful implementation of LST initiatives

\subsection{A whole-of-system quality improvement approach and New Zealand's System Level Measures} programme

In 2007, the Institute of Health Improvement (IHI) published a white paper that described and promoted the use of a small set of whole-of-system measures that were not disease- or condition- specific, to measure the overall quality of a health system and align improvement initiatives across the continuum of care [38]. The system-level measures were based on the IOM's six dimensions of quality. The whole-of-system measures approach aimed to support strategic quality improvement planning and complement the traditional performance management metrics used in health systems [38]. This white paper formed the blueprint for New Zealand's System Level Measures (SLM) programme, which began in 2014 as the Integrated Performance Incentive Framework (IPIF) focusing on primary care [39]. In 2015, the IPIF evolved to a wholeof-system focus and was rebranded as the SLM programme.

The SLM programme is an example of an LST initiative and was designed to enhance a collaborative way of working beyond organisational and professional boundaries, address health inequities and encourage continuous learning and quality improvement [15]. The programme consists of a small set of high-level health outcome measures co-designed with the health sector, which provide a framework for local quality improvement. Improvement actions are determined 
locally by frontline health care professionals working together with their managers and communities. There is also a set of contributory measures that support continuous learning; these are activity and process measures chosen locally to monitor progress against improvement actions [40].

The SLM programme was developed by New Zealand health system leaders from the Ministry of Health (MoH), District Health Boards (DHBs), and Primary Health Organisations (PHOs) [41]. The philosophy of the programme is embedded in the 'new power' approach that focuses on local, clinically led continuous improvement from past performance and a collaborative way of working through DHB and PHO Alliances to create a collective sense of purpose and joint accountability. The key purpose of the SLM programme is to achieve the Triple Aim: improved quality, safety and experience of care; improved health and equity for all populations; and best value for public health system resources [40]. The programme was implemented in July 2016 and its use of highlevel health outcome measures and a collaborative way of working using Alliances goes beyond the aspiration of the IHI's white paper [41].

Alliances are informal networks in the New Zealand health system that were introduced to integrate the planning and delivery of health care between DHBs and PHOs [42]. These networks operate with support from DHBs and have varied membership. Some Alliance leadership teams comprise senior leaders from DHBs and PHOs. Others have a broader membership that may include community providers, Māori and Pacific health providers, pharmacy, ambulance, patient advocates, Iwi and local communities [43].

Initial implementation of the SLM programme requires a set of key elements and the right contextual factors to facilitate a collaborative way of working between agents in the system to develop a high-quality integrated plan and the ability to fully implement the plan in their districts. The agents include senior operational and clinical leaders, frontline health care staff, managers, and whānau, communities and Iwi. A high-quality integrated plan is developed through clinically led processes involving all agents, using evidence from health and social data, and have improvement actions that should change health outcomes [40]. This planning process, along with the full implementation of the plan and the ability to undertake continuous reflective learning, encompass successful implementation of the programme.

Four years on, the SLM programme continues to be an important part of the New Zealand health system's quality improvement and integration agenda [15]. The programme has evolved with 
subsequent changes based on feedback from Alliances and those involved in the implementation of the programme.

\subsection{Gaps in New Zealand health system transformation knowledge}

I have led the SLM programme since its inception and therefore have inside knowledge about the implementation of the programme. Having led the programme from the centre (the $\mathrm{MoH}$ ) for four years placed me in a unique situation of observing and experiencing the conditions that enabled and constrained the implementation of the programme in different DHB districts. I also observed that health system leaders are overwhelmed with change management theories and organisational changes and lack common understanding on the programme architecture that underpins efforts to successfully implement LST initiatives (such as the SLM programme) in the New Zealand health system.

New Zealand has its own research to support health policy, necessary because of its unique demographic profile, health system funding and organisational arrangements, geographical contexts and Te Tiriti o Waitangi as a foundational document. There is available research about the impact of the health system structure, funding, national policies, and success of small-scale initiatives on the delivery of health care and health outcomes. However, nothing has been identified in the literature that explicitly reveals the architecture that underpins efforts to successfully implement LST initiatives that involve multiple organisations in a complex system.

\subsection{My motivation for the research}

My main motivation for this research was to build on the early insights I had gained from the SLM programme and to capture in more depth the knowledge and experience of those working on the programme and those with knowledge and experience about how to successfully implement LST initiatives. Much about the SLM programme and the knowledge and experience of its development and implementation is not published and remains in the heads of individuals. This research provided an opportunity to describe the SLM programme, to reveal the insights and experiences from its development and implementation, and to describe and analyse the programme architecture that underpins efforts to successfully implement LST initiatives, such as the SLM programme. Health system leaders and informal networks, such as Alliances, could then use this research literature to successfully implement future LST initiatives to bring about the desired system transformation. Such research-based insights are increasingly recognised as being more useful to system leaders in a complex system than systematic reviews, pilots or randomised 
control trials because these insights shift leaders' thinking from a linear cause-and-effect paradigm to one that recognises multi-causation effects and also because they take the influence of context into consideration when thinking about change $[1,37,44-46]$. These insights also support health system leaders to think beyond organisational reforms and 'new public management' ideas that have led to transactional relationships in the health system, and to embrace 'new power' values that involve collective action to achieve a shared vision [32].

While being an 'insider' offered me first-hand experience and access to information and people, my dual role as an employee of the MoH leading the SLM programme and as a researcher created an ethical challenge for the research. Nevertheless, it was important for me to locate lessons from the SLM programme in the wider academic literature and ensure there is a written record for others to refer to in the future. The research also provided an opportunity to bridge the gap between health researchers who are often producers of research and health system agents who are the consumers of research. I acknowledged the ethical challenges in full and mitigated these through several strategies. The ethical considerations for the research are discussed in detail in Chapter 8.

\subsection{The research approach}

Greenhalgh and Papoutsi [6] emphasise that research on LST initiatives, where both the interventions and the system these are implemented within are complex, requires a complexityinformed approach. A complexity-informed approach provides a deep understanding of the health system dynamics and the effects of contextual factors and adopts a continuous learning approach that recognises and adapts to the changing relationships and interactions between the agents in the system.

The application of a realist logic of enquiry, nested within the macro framing of complex adaptive systems, is well suited to complexity-informed health services research because it provides a sound framework to study how contexts and mechanisms influence the implementation and the outcomes of complex programmes and uses individual experiences to reach a closer understanding on how and why programmes work (or not) [47]. Realist research starts with midrange or programme theories formulated from past experiences and previous research and ends with refined theories that describe the context-mechanism-outcome interplay for the programme, initiative or intervention [48]. 
This thesis investigated the journey of the SLM programme, which is unique in its collaborative design with DHB and PHO leaders, its continuous improvement approach and its implementation through Alliances. The research uncovered the success as well as the challenges of the programme and used the knowledge of senior system leaders whose behaviours and insights are crucial in bridging the gap between the theory of LST initiatives and the reality of successfully implementing these initiatives in the New Zealand health system.

In realist research, prior knowledge of the research subject matter and purposeful selection of participants that are 'information rich' are essential tenets $[4,49]$. This allows the researcher to use personal experience to identify and test theories from within the system rather than 'looking into' the system. Therefore, my inside knowledge of the health system, and three years of firsthand experience of observing the key elements that support implementation of the SLM programme and how contexts influenced the successful implementation of the programme, was invaluable to the research.

The research breaks new ground with the creation of a self-assessment maturity matrix using the knowledge of senior system leaders. The maturity matrix provides a national tool that supports conscious and deliberate learning of collaborative networks, such as Alliances and locality networks. The tool allows these organisations, to assess their readiness for change and build their capacity and capability to adopt complex innovations (such as LST initiatives) and sustain these long-term at an organisational and system level. 
PART A: BACKGROUND

AND LITERATURE

REVIEW 
Part A of the thesis contains four chapters.

\section{Chapter 2: The New Zealand Health System}

This chapter describes the Westminster-derived New Zealand health system: how past reforms and the 'new public management' ideas have shaped its current structure, the quality of care delivered to New Zealanders, its current performance and upcoming planned reforms. This description provides the setting for the research and highlights that while the health system works for most New Zealanders, there are persistent health inequities for Māori (the indigenous people of New Zealand) and Pacific populations. The chapter also describes the evolution of New Zealand Alliances, who are responsible for leading the implementation of the SLM programme, and the alliancing way of working that is challenging the 'new public management' ideas to improve system performance through collective action.

\section{Chapter 3: The New Zealand System Level Measures Programme}

This chapter outlines the development and implementation of the System Level Measures (SLM) programme, and my influence and insights as the National Programme Manager leading the implementation of the programme. The SLM programme was developed by the $\mathrm{MoH}$, together with DHBs and PHOs, and is an LST initiative aimed at health system improvement and integration. The programme was implemented in July 2016 and continues at the time of writing this thesis. The key purpose of the programme is to enhance a collaborative way of working beyond organisational and professional boundaries, address health inequities and encourage continuous learning and quality improvement to achieve the Triple Aim.

\section{Chapter 4: Academic Context for the Proposed Research}

This chapter applies complexity thinking to health systems and describes health systems as complex adaptive systems. These systems are emergent, unpredictable and generative and need to be transformed, via LST initiatives, to deliver seamless, patient-centred, holistic and equitable health care. The chapter introduces the concepts of 'new power' and 'old power' and argues how New Zealand's Westminster-derived system and 'new public management' ideas both constrain and enable implementation of LST initiatives in health systems. Finally, the chapter reviews the literature on the use of maturity matrices in health care settings and highlights how maturity matrices with a self-assessment approach can be used as a tool to support the notion of a learning system. 


\section{Chapter 5: Research Issues, Aims and Questions}

Part $A$ ends with Chapter 5 that summarises key gaps identified in the existing literature in Chapter 4 and outlines the programme architecture that underpins efforts to successfully implement LST initiatives derived from my insights and the literature, and research aims and questions. 


\subsection{THE NEW ZEALAND HEALTH SYSTEM}

The purpose of this chapter is to understand the aspirations of LST initiatives, such as the SLM programme, and the operating environment that enables and constrains the successful implementation of these initiatives. The chapter does not aim to provide a comprehensive view and discussion of the complexities of the New Zealand health system, as that would be a thesis in itself, but rather it emphasises the key aspects of the system relevant to the research. There is a focus on primary care reform as this is where both the complexity is and where the more significant opportunities are for system transformation. Increasing healthy life expectancy for the New Zealand population through prevention will require more preventative health care delivered in primary and community settings [18]. Both early access to and better management of health care in primary care are likely to reduce costly hospital care. Hospital care is usually delivered in hospitals that are owned and managed by a single provider and with a single contractual arrangement. In contrast, health care in primary and community settings is delivered by multiple providers that often have a mix of government and private funding and often through multiple contractual arrangements. The organisational, funding and contractual arrangements make delivery of co-ordinated care challenging with providers trying to meet the needs of their patients and communities, deliver on their contractual accountabilities, maintain organisational autonomy and be financially sustainable. Health system leaders have to find ways to overcome these challenges to deliver health care that is patient-centred and equitable.

\subsection{A Westminster-derived system of governance}

New Zealand has a Westminster-derived constitutional system in which the government is formed with the consent of people under a Mixed Member Proportional (MMP) voting system [50-52]. In the MMP voting system, voters have two votes: an electoral vote for a local candidate, who becomes a Member of Parliament (MP) after winning most votes in their electorate; and a party vote that decides the total number of seats each political party gets in the House of Representatives (the House) [52]. For political parties to have seats in the House, they must either get at least five percent of the party vote or win an electorate seat [52]. The proportional system means that the proportion of party seats in the House is about the same as the proportion of party votes. The government is formed by the political party or groups of parties with most seats in the House; usually this requires coalitions or agreements between two or more political parties [52]. The House is elected for a maximum three-year term [52]. 
Ministers are appointed from Members of Parliament (MPs) in the governing parties and the government is held to account formally by opposition MPs and Select Committees [51].

Cook and Huges [51] state that "it is fundamental to democracy that politicians can be elected from ordinary citizens, without a need to demonstrate eligibility by meeting prior tests of skill, aptitude, judgement or even community standards of integrity" ( $p 35$ ). This means that most MPs have never worked in the areas of which they are in charge; most Ministers of Health, then, have neither worked in the health system nor are familiar with its complexity. They rely on advice from the $\mathrm{MoH}$, and the Ministers' own political advisors. Ministers' decision-making is in response to the priorities of their government and their campaign promises, and is also influenced by the media, their constituents and powerful organisations that are often acting in their own selfinterest.

The authority for government flows through Cabinet, which sets the priorities for legislation and directs and controls national policies. The ability for the political system to react and respond to social and economic context is a major benefit of the Westminster-derived system. In New Zealand, the unicameral legislature, a three-year term, and with governments typically holding a majority in the Parliament mean that governments can implement policies fairly quickly so they can demonstrate their achievements to their constituents [51,53]. The three-year term means that governments can have a short-term focus on the efficiency of the public sector organisations rather than on the usefulness or effectiveness of policies [51]. This political environment makes public sector organisations vulnerable to regular reforms with changes in governments, albeit the introduction of the MMP voting system in 1996 has tempered extreme policy swings between successive governments [53].

\subsection{The health care system in New Zealand}

\subsubsection{Creation of a universal health service}

New Zealand was the first democratic country to create a universal national health service $[24,54]$. The goals of the 1938 Social Security Act were for all New Zealanders to have equitable access to health care that had a preventative focus and was integrated between primary and hospital services $[24,54,55]$. The implementation of the Act, however, separated the planning, funding and provision of services for public health, primary care and hospital care [21,24], leading to different planning and funding arrangements for different services, fragmenting service delivery. 
The Department of Health was responsible for public health services that were delivered through 18 district offices. Primary care services were funded by the Department of Health and delivered through privately-owned small general practitioner-led teams. Diagnostic tests and pharmaceuticals including dispensing services were delivered by privately owned organisations. Local publicly-owned hospitals provided specialist inpatient, out-patient and some communitybased services [21,24]. Non-government organisations (NGOs) delivered a range of communitybased services; for example, Plunket, which provided well child services; while IHC and CCS for children delivered health services for children with intellectual and physical disabilities [21].

The influence of the then very powerful medical profession opposed the government's proposal of providing fully-funded primary health care based on a capitation model $[24,54,55]$. The medical profession believed that involvement of the government as a third party would undermine the doctor-patient relationship $[54,55]$. So, while the introduction of Social Security Act led to free hospital and maternity care, provision of primary care through general practitioners included a co-payment from service users [21,24]. This patient co-payment scheme remains in place today.

General practice teams are the first point of contact in the health system and are gatekeepers to other primary care services (e.g., medicines, laboratory tests), secondary care and some community services. Most general practices are owned by independent general practitioners (GPs); general practice teams comprise three or more GPs, at least one practice nurse and other support staff [56]. A few general practice teams are led by nurse practitioners; this is common in rural areas where recruitment and retention of GPs is challenging [10].

Community providers and NGOs provide health promotion, community mental health, disability, well child, ambulance, pharmacy and aged care services, and include Māori-led and Pacific-led providers [10]. There are variable co-payments charged to patients to access some of these services such as prescribed pharmaceuticals, aged-residential care and ambulance services.

In 1974, the Accident Compensation Commission (ACC) was established to manage a no-fault compensation scheme for accidents and injuries [57]. The scheme has evolved over the years and continues today. It is an insurance scheme, with funding from a range of levies (employees, employers, car registrations and petrol). It is provided on a fee-for-item-of-service basis by health providers registered with the ACC (those same providers deliver government funded health services as well). ACC benefits include hospital and medical expenses, rehabilitation and transport costs, weekly compensation for injured workers, funeral costs, and one-off payments 
for permanent and mental injuries and accidental deaths. All New Zealanders, including visitors, are covered by the scheme [57].

New Zealanders can also purchase private health insurance, choosing to avoid waiting lists of public hospitals for non-urgent care and to access services not provided in publicly funded health care, such as non-urgent elective surgeries that people do not qualify for through the publicly financed system or services such as dental care [10]. Services are delivered through a small number of privately-owned hospitals; New Zealanders can also choose to pay for such services themselves.

The health care model in place from 1940s to 1980s was regularly criticised for not enabling an environment for providing co-ordinated care, given its separation of planning, funding and delivery of different forms of care, and a lack of information sharing between providers [21]. The co-payment scheme led to many concerns about poor equity of access to primary care services, owing to constant rise in service user fees over time [21]. These concerns, amongst others such as, poor quality of care owing to a lack of co-ordination in planning and delivery of health services, waste of scarce health resources owing to duplication of care, and harm to patients from the use of incompatible medications [21], led to major system reforms in the 1980s and 1990s.

\subsubsection{The 1980s and 1990s reforms}

The first major set of organisational health care reforms began in the 1980s with the establishment of 14 geographically-based Area Health Boards (AHBs) whose role was to coordinate the planning and delivery of health services to their geographically defined populations $[21,24,58]$. The reforms linked the roles of the 18 district offices of health with mergers of previously 31 local hospital boards [55]. The reforms also introduced a Charter of Health, a set of national Health Goals, and an early form of contract between central government and the AHBs, to increase accountability to central government. Although AHBs were to plan for primary care services, in practice, the funding for primary care remained separate and in the hands of the Department of Health [21].

The second set of reforms in the 1990s aimed to improve access to health services and the efficiency of the system $[21,22,24,56]$. AHBs were disestablished, and four Regional Health Authorities (RHAs) were created to integrate all health services planning and funding functions. The 1990s reform separated the planning, funding and purchasing roles from the health service delivery roles and promoted competition for the latter. RHAs could choose how best to allocate resources across public health, primary care, secondary care and disability support services, and 
all providers were to be funded and held accountable through formal contracts. The publicly owned hospitals that were part of AHBs became 23 Crown Health Enterprises (CHEs), which competed with each other and with other providers (e.g., privately owned hospitals) for funding from the RHAs [24,59]. CHEs were to make a surplus, to be reinvested in the sector. The (centreright) government had hoped that competition would improve the quality of health care delivered, improve efficiency and reduce health care expenditure $[22,56,59]$. However, the separation of roles led to contracting complexities in a small country and the competitive model provided fewer incentives for health service providers to work collaboratively to deliver coordinated, patient-centred care [21]. The large savings promised by the reforms did not eventuate and there were significant concerns from health care professionals and the public regarding the cost and competitive incentives embedded within the model $[24,56,59]$.

The national election in 1996 changed the political makeup of the government with the introduction of the MMP voting system. The influence of a minor party as part of the government led to CHEs being replaced by Hospital and Health Services (HHSs) and the surplus requirement removed [24,56,59]. The four RHAs were amalgamated into a single national Health Funding Authority (HFA) and the focus shifted to greater collaboration, integration of primary and hospital services, better access to services, improving the provision of primary care and reducing health inequities $[21,22,24]$.

Three unexpected and positive outcomes from reforms of the 1990s were the emergence of the general practitioner-led networks, the increase in the number of Māori and Pacific health providers, and the creation of the Pharmaceutical Management Agency (PHARMAC) [21,59,60]. The latter was successful in reducing pharmaceutical-related health expenditure and continues today [24]. The general-practitioner-led networks brought general practitioners together to facilitate the contracting of primary care services and to increase peer support and quality improvement initiatives $[21,60]$. Later these networks broadened to provide administrative and clinical leadership functions for general practice teams and provided the platform for the later creation of meso-layer PHOs to manage and deliver primary care services $[21,60]$.

\subsubsection{The early 2000s reforms}

\section{District Health Boards and their roles}

A change in government (to a centre-left coalition government) in 1999 brought further organisational reforms to restore a non-commercial health system that involved local communities in the planning and management of health services [56]. The New Zealand Public 
Health and Disability Act was introduced in 2000, which led to the formation of 21 District Health Boards (DHBs) with locally elected boards, that replaced the HFA and the HHSs $[22,24,55,56]$.

DHBs are legislated to improve, promote and protect the health of people and communities, promote integration of health services, seek optimum arrangement for delivery of most effective and efficient health services, reduce health disparities and improve health outcomes for Māori and other high priority population groups, and foster community participation in planning for delivery of health services and health improvement [61].

The creation of DHBs decentralised the planning and decision-making roles for most services and re-integrated the planning, funding and delivery of hospital services $[21,22,56]$. Some services were centralised and became the responsibility of the $\mathrm{MoH}$, which are purchased directly from providers, including, public health services, midwifery services, post-natal care of babies, and disability support services for those aged under 65 years $[56,59]$.

DHBs remain in place today, with 20 DHBs (two DHBs merged reducing total number to 20) overseeing health services in their districts and most government health funding is distributed to DHBs using a weighted population-based funding formula [56].

DHBs provide and manage health services for their populations through public hospitals using their provider arms, where care is free for those eligible for publicly funded health care services [10]. DHBs at times contract private hospitals to assist in the delivery of care, especially elective care. DHBs plan, purchase and manage primary and community health services for their populations through their planning and funding arms, contracting with a range of providers to deliver care: the main one being through the PHOs.

\section{Primary Health Organisations and their roles}

In 2001, the Primary Health Care Strategy, based on principles of the Alma-Ata Declaration [62], was released leading to the creation over time of $80+$ PHOs $[21,22]$.

PHOs are not-for-profit meso-layer organisations that are funded by DHBs to provide comprehensive primary care services through their member general practices. Citizens can choose the general practice they enrol with and general practices choose the PHO they become members of [10].

PHOs are funded using a weighted capitation formula based on their patient enrolments and taking into consideration age, gender, high user status and rurality for first level services [10]. 
Additional capitation funding is used to enhance equity and access of essential primary health services by lowering the cost of first level services through government subsidised initiatives such as the 'very low-cost access' (VLCA) scheme, Community Services Card (CSC) funding and zero fees for children aged under 14 years [10].

PHOs pass on funding for first level services to their member general practices using the same weighted capitation formula. The funding arrangements in New Zealand means that general practices retain the right to charge patients co-payments to access primary care services. The amount of fees charged is decided by general practices with limited rules around frequency at which general practices can increase their fees and the maximum annual percentage increase as agreed with their contracted PHO and DHB [10].

PHOs pass on additional capitation funding for government subsidised initiatives and this funding is allocated to practices on the condition that general practices maintain their co-payments below an agreed threshold [10].

Since 2013, PHOs and general practices have had access to a flexible funding pool, which is allocated in agreement with the DHBs that PHOs contract with. The funding is intended for PHOs to use to improve access to primary care services; to provide intensive clinical management of chronic health conditions in primary care through the Care Plus Service; to deliver health promotion services; and to implement Alliance recommendations (Alliances are discussed later in section 2.3) [63]. The funding is also used by PHOs to provide management services for their member general practices [63].

There is also performance incentive funding available to PHOs and their member general practices. Initially this funding was used to support the PHO Performance Programme (PPP). Later this funding was re-purposed to support the implementation of the SLM programme [63].

Tension between successive governments and GPs have remained with the dichotomy of the publicly financed-privately provided model. This has at times resulted in a lack of positive engagement from GPs to implement the Primary Health Care Strategy (PHCS) [21] and the minimal direct influence of GPs in the design and implementation of primary care policies [56].

\subsubsection{The late 2000s and 2010s}

The re-election of a centre-right government in 2008 shifted the focus from local decision-making and reducing health inequities to a focus on clinical engagement, productivity and quality improvement, including integration of care. The government commissioned a Ministerial Review 
Group, which predominantly comprised medical professionals, who criticised the system for being overly bureaucratic with an inadequate focus on frontline services and a lack of national co-ordination around quality and service integration $[22,56]$. The review led to creation of the National Health Board, which became responsible for operational oversight of DHBs' performance. The National Health Board was physically located as a business unit in the MoH but reported directly to, and was directly accountable to, the Minister of Health. The review also led to new organisational arrangements to reduce bureaucracy with mergers of 'back office' (e.g., planning and funding) functions of DHBs, and PHOs were encouraged to amalgamate to strengthen their capability and free up funding for frontline services, which over time led to a reduction in the number of PHOs from 80 to 36 [22,56]. There are now 30 PHOs and with few exceptions, all general practices are part of PHOs [10].

While the implementation of the PHCS initially led to reductions in the cost of access to care and increased utilisation of primary care services, there were a number of concerns about how DHBs and PHOs worked together to deliver integrated care, and that health care delivery was barely changing at all and remained fragmented $[64,65]$.

There was a lack of clarity on the remit of PHOs, in particular how the role of PHOs differed from those of DHBs [64]. Both have the core role of planning and funding primary care; however, DHBs have a statutory function for their local population whereas PHOs are not required to take responsibility for geographically defined populations. PHOs are responsible for their enrolled populations and multiple PHOs operating in some districts complicated the way DHBs worked to plan and deliver primary care for their geographically defined populations [64].

PHOs believed that DHBs were investing in their hospitals at the expense of primary and community services [59]. PHOs struggled to integrate care across primary, hospital, and community care owing to a limited leverage over the allocation of funding to providers [64]. GPs felt disenfranchised during the implementation of the PHCS and became disengaged from planning and implementing change [65].

The review of the implementation of the PHCS recommended a focus on strengthening relationships between the government and general practice, and for PHOs to focus on strengthening clinical engagement and leadership [64]. The review also recommended better investment in IT infrastructure to enable delivery of co-ordinated care. 
In the 2010s, policy attention shifted to encouraging new models of care that were patientcentred and delivered seamless care 'closer to home' and the alliancing model was introduced to integrate primary and hospital care [21].

The National Health Board was disestablished in 2015 with its functions incorporated back into the $\mathrm{MoH}$. Appendix 1 shows the current structure of the New Zealand health and disability system [66].

\subsection{Alliances}

All health systems contain various models of governance with each health service provider having its own governance arrangements, for example, DHB boards. Additionally, the system has institutional arrangements such as professional associations that represent the interests of health care professionals. According to Gauld [43], these governance and institutional arrangements rarely foster integration across the system.

The alliancing model of governance encourages vertical (intra-organisation) and horizontal (interorganisation) integration with the aim of bringing different actors present in a complex system together to achieve jointly agreed outcomes $[43,60,67]$. The model originates from the construction industry, where Alliance contracting is used to bring individual companies to work together to achieve their common goal, on time and on budget [43]. The model relies on a collective contract with pre-agreed gains and losses in which 'everyone wins, or everyone loses' and success is dependent on the overall performance of all parties to the Alliance [68].

Alliances are informal trust-based networks; an alliancing way of working involves unprecedented levels of trust, mutual understanding, informal rules, guided by commitment among partners to work towards a shared vision and overcome great challenges in a complex system that no single player is likely to achieve individually; and that requires inter-organisational co-operation [68-71]. Alliances, when underpinned by commitment and trust among partners, produce co-operative behaviours of knowledge sharing, knowledge transfer and less resistance to change [71].

Alliances are similar to other informal trust-based networks used in health systems worldwide to deliver integrated patient-centred health care. These networks are often referred to by different names; such as integrated care systems or networks [72], accountable care organisations [73], and place-based or place-led partnerships [74]. These networks have the ability to take on complex issues and foster integration and local innovations. Evidence in literature emphasises 
that it is the depth of processes, a high-level of trust, strong relationships and the constant feedback loops that add value to these networks $[67,69,71,75]$.

The National Health Service (NHS) England and NHS Improvement believe that for such networks to be successful, they need to be supported with a similar network at the system level, so it has a "systems within system" approach [72]. They refer to systems, places and neighbourhoods where the system level sets and leads the overall strategy and creates learning communities, and the places and neighbourhoods are closer to the populations they serve, designing and delivering services that meet the needs of the local populations [72].

In New Zealand, Canterbury DHB was the first to use the alliancing model through the Canterbury Clinical Network (CCN) using Alliance contracting early in their system transformation journey (prior to 2010) to deliver integrated care [68]. The model was then used in 2010 with the Better, Sooner, More Convenient (BSMC) primary care initiative. Alliances were to be used as governance networks, made up of DHBs and PHOs, to deliver the BSMC objectives of delivering care closer to home; improving health outcomes; and reducing pressure on hospitals [76].

Since 2013, the MoH has contractually required all DHBs and PHOs in each district to form Alliances through the Operational Policy Framework (OPF) [2] and the national PHO Services Agreement [63]. The OPF contains business rules, policy and guidelines that outline operating functions of DHBs. It is endorsed by the Minister of Health and DHBs are responsible for complying with the OPF [2]. The national PHO Services Agreement sets out the roles, responsibilities and accountabilities for DHBs, PHOs, and contracted providers (GP practices) to fund and deliver primary health care services [63].

The aim of the alliancing model is to bring together all actors present in the health system to improve health outcomes by providing "integrated and co-ordinated health services through clinically-led service development" (p 1) [42]. The alliancing model provides a practical platform to involve clinical leaders (not holding formal leadership roles in their employer organisations) in decision-making about the planning and funding of health care [43].

The Alliance Charter, published by the $\mathrm{MoH}$ [42], outlines the rules of engagement that Alliance members pledge to (Box 2.1). It also outlines members' "commitment to act in good faith to reach consensus decisions on the basis of "best for patient, best for system'"' (p 1) [42]. Members commit to engage actively in good faith, and honour confidentiality, shared responsibility, shared decision-making, and shared accountability to enable open and transparent discussions [42]. 
New Zealand Alliances are not legal entities and therefore cannot commission services nor do they have their own budgets for spending on health care. Instead, Alliances operate with support from DHBs and members define a work programme and agree on a shared vision and goals for their local health system with their DHBs, including a budget to deliver their work programme; using the flexible funding pool [42]. Gauld [43], in his review of the theory and practice of integrative health care governance, highlighted the importance of Alliances being supported by DHBs with a dedicated budget that Alliance leadership team (ALTS) can access to achieve the Alliance work programme.

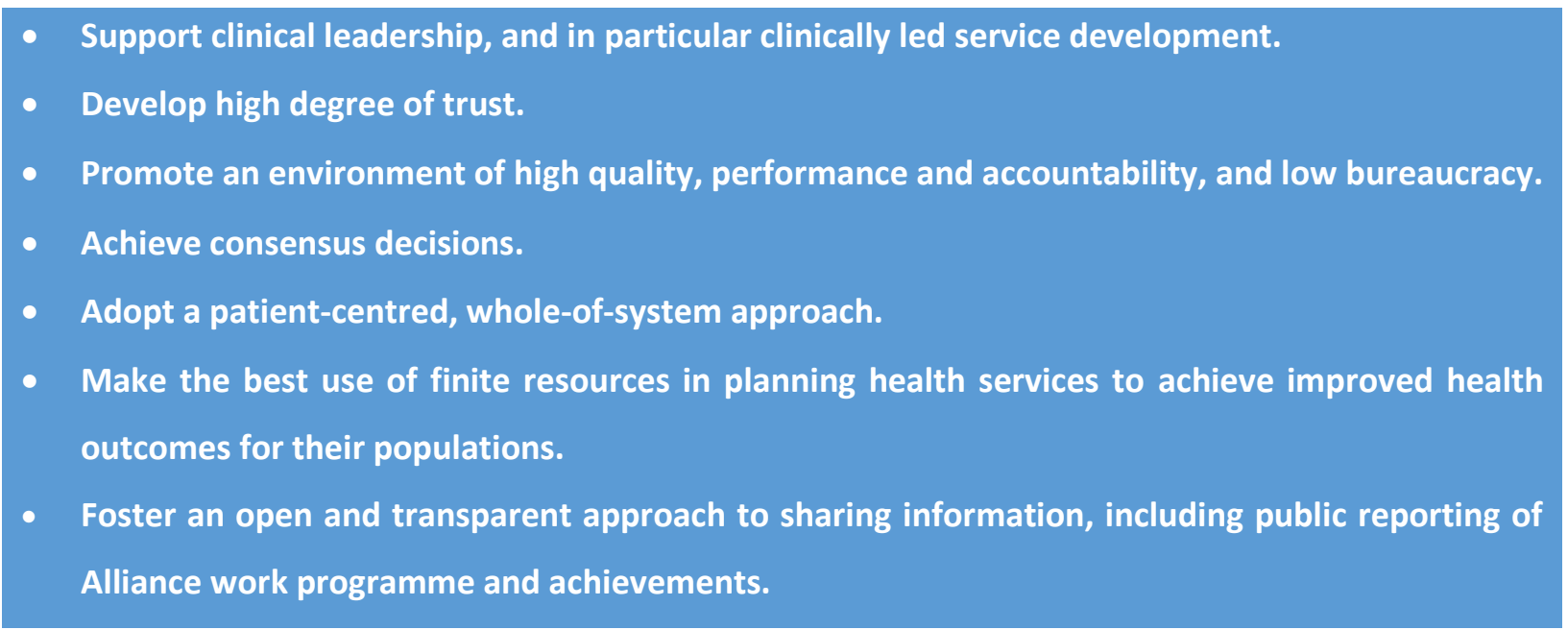

Box 2.1: Alliancing principles [42]

Alliancing is underpinned by the principle that members are appointed to work on behalf of the patients and the local health system and therefore have patients and equity at the centre of all their decisions [43]. Gauld [43] emphasises that members are not representatives of their organisations or professions and getting the right people who understand and value the alliancing concept is paramount. Gauld [43] also stresses that an independent chair is important to ensure that the Alliance remains focused on achieving the shared goals and that the agenda is not dominated by one partner.

The ALT provides high-level governance and is made up of senior operational and clinical leaders from DHBs and PHOs and may include local Iwi, community representatives, and other health service providers such as ambulance services, pharmacy and Māori and Pacific health providers [43]. The most common type of ALT in New Zealand consists of DHB and PHO senior operational and clinical leaders, as per the contractual requirement.

Alliances establish service level Alliance teams (SLATs) or other informal working groups, such as consumer councils and clinical leadership forums, as necessary to deliver on the Alliance work 
programme [42]. The SLATs are workstreams within the Alliance structure (e.g. child health SLAT, youth health SLAT, rural SLAT) and report to the ALT. The SLATs consist of a diverse group of people that may include clinicians, managers, analysts, service users and local Iwi representatives and use improvement science methods to identify problems and co-design solutions to improve health service delivery.

Commentators have emphasised that it is critical that local Iwi should be members of both the ALTs and the SLATs to demonstrate a true Te Tiriti partnership [77,78]. Similarly, involvement of whānau and communities is critical at both levels to ensure that focus remains on people and equity $[69,78]$. In practice, the membership of the ALTs and the SLATs is determined by DHB and PHO senior leaders and varies across New Zealand depending on local relationships and a willingness to engage with broader system partners.

The ALTs consider the recommendations of SLATs and then make recommendations to the DHB executive team on the activities and services to meet the Alliance vision and goals [42]. The DHBs commission change through their contractual processes to give effect to the Alliance priorities. The ALTs monitor outcomes of Alliance activities and feed the information back to system stakeholders and refresh their work programme and membership at least annually [42]. Figure 2.1 illustrates this continuous alliancing way of working.

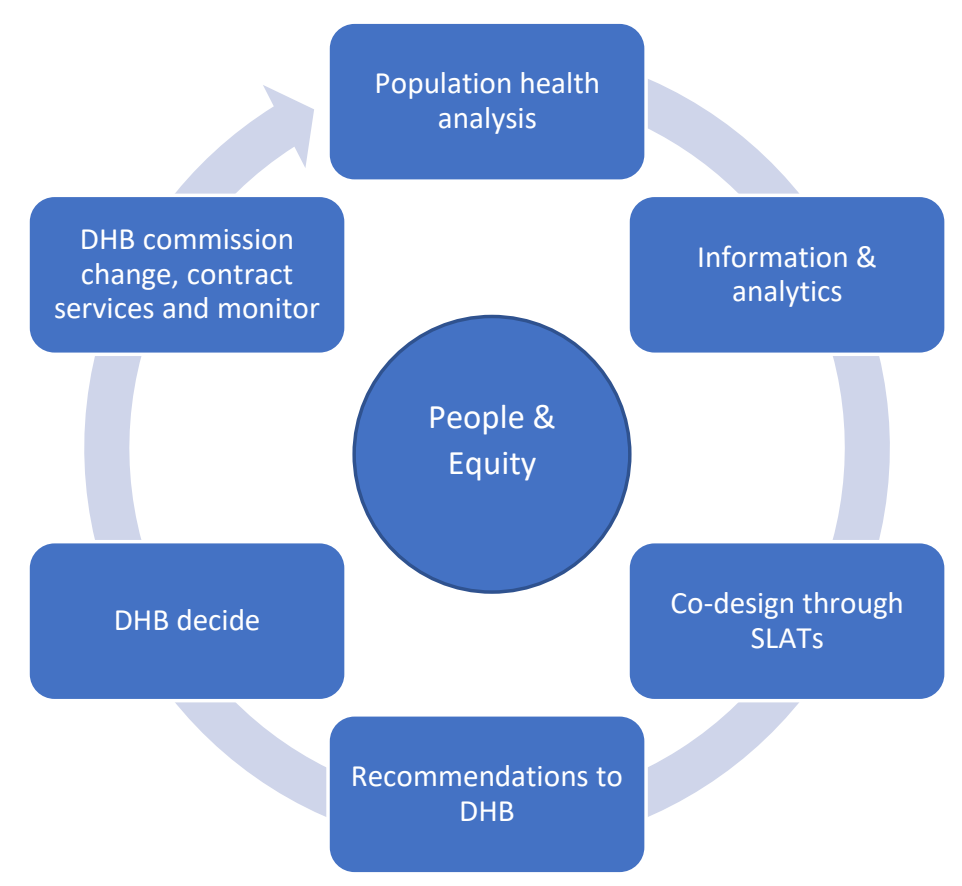

Figure 2.1: The continuous alliancing way of working 
New Zealand Alliances have adapted over time, depending on local relationships, interactions, behaviours and the history of different organisations working together; they therefore vary across the country in form, function and maturity. Some DHBs have a single Alliance with all their health system partners, while others have multiple Alliances both within and across DHB districts. The latter is more prominent where PHOs provide primary care services in more than one DHB district.

Alliances are an important context for this study because these networks are responsible for leading the implementation of the SLM programme. This responsibility was placed on Alliances to experiment with innovative ways of bringing key system agents together to build new collective forms of accountability for implementation of an LST initiative. The understanding of how networks such as Alliances support and constrain successful implementation of LST initiatives is particularly important to health system leaders given the current and future challenges facing the New Zealand health system.

\subsection{The role of 'new public management' in health system reforms and performance management}

'New public management' (NPM) was an international agenda for public sector reforms to counter the traditional bureaucratic public administration paradigm and improve the efficiency and accountability of public sector organisations, and was firmly embedded in many countries by the end of the $20^{\text {th }}$ century $[79,80]$. NPM drew on principal-agent theory where agents faithfully follow the instructions of the principal to achieve objectives set out by the principal and value for money was to be demonstrated through measurement of performance using clearly defined quantitative targets [80]. Hood [79] introduced the seven key doctrines of NPM; these are outlined in Table 2.1 
Hands-on professional management Clear accountability and control of organisations. Actions cascade from those holding formal leadership roles at the top. The 'golden thread' approach.

Explicit standards and measures of Clearly defined quantitative performance measures and performance targets to demonstrate efficiency of organisations.

Greater emphasis on output controls Focus on results rather than processes. Allocation of resources and rewards and linked to performance. The 'carrot and stick' approach.

Shift to disaggregation of units in the Machine view of systems in which the system is broken into public sector parts that can be managed with using one-line budgets. The separation of planning and provision of services.

Shift to greater competition in public Using competitive procurement models to gain efficiency in sector the system.

Stress on private sector styles of Move away from 'old management' of military-style practice management practice to integrate private sector management tools.

Stress on greater discipline and Reducing compliance and administration costs ('back-office' parsimony in resource use functions), 'living within your means', resisting union demands.

Table 2.1: Doctrines of 'new public management' reproduced from Hood [79]

The United States, the United Kingdom, Australia and New Zealand were early adopters of the NPM movement and NPM ideas informed the 1980s and 1990s reforms of the New Zealand health system [81]. With the Westminster-derived system and the fact that much of the New Zealand health system is publicly funded and owned, these ideas were already reflected in the hierarchical structure of the health system, however, they became more dominant with the separation of planning, funding and delivery functions, and the competitive and performanceoriented model brought in by the 1990s reforms [81].

NPM ideas also became embedded into the performance management processes of public sector organisations in New Zealand, which comprise performance agreements between the responsible Ministers and the Chief Executives of public sector organisations $[51,81,82]$. These agreements list the outputs and outcomes to meet the legislative requirements of the State Sector Act 1988 and the Public Finance Act 1989, and Cabinet requirements. The outputs and outcomes are agreed by the Cabinet through an annual budget process and cascade down to public sector organisations through the lead advisors of the government: the Office of the Auditor-General, Department of Prime Minister and the Cabinet, and The Treasury [50,51]. This process is referred to as the 'golden thread' approach in which national performance measures are linked to local performance measures and information used for external accountability is consistent with information used for organisation learning and improvement [50]. The State 
Services Commission (now known as the Public Service Commission) monitored outputs and performance of Chief Executives [82].

According to Gill [50], the 'golden thread' approach aligned with a command and control leadership style rather than a drive for continuous improvement because information used for learning and improvement are not suitable for external accountability. Public sector agencies have tended to operate individually, disconnected from each other. The command and control leadership style tends to cascade to the central agencies such as the $\mathrm{MoH}$ [50].

Critics of the 'golden thread' approach point out that the disconnection of public sector agencies and the command and control leadership style led to a lack of integration across the agencies to deliver a collective impact for citizens and communities $[51,81]$. The annual process leads to a lack of long-term investment to achieve outcomes and there is no recognition of the complexity of public sector agencies [51]. Further, the lack of in-depth policy evaluation and a lack of commitment to continuous improvement has contributed to long-term issues such as institutional child abuse, leaky buildings, housing and water quality issues, and health infrastructure that is no longer fit for purpose [51].

In the health system, NPM ideas led to the introduction of national performance measures for DHBs and PHOs $[22,24,83]$. The measures used have altered over the years with change of governments, however, the overall approach has remained.

The PHO Performance Programme (PPP) was a pay-for-performance programme that began in 2005 to improve the health of enrolled populations, reduce health inequities and reward quality improvement within PHOs [83]. The programme was supported by incentive payments paid to PHOs, based on the rate of improvement being made; there were higher payments for improvement in the higher need populations such as Māori or Pacific populations or those living in the most deprived areas [83]. The performance of PHOs on some of the measures were publicly reported.

From 2009 to 2017, six national health targets were used to publicly report DHB performance: improved access to elective surgery, shorter stays in emergency departments, increased immunisation for babies aged eight months, raising healthy kids, shorter cancer treatment times and better help for smokers to quit $[84,85]$. PHO performance was also publicly reported for two of the health targets: increased immunisation and better help for smokers to quit [86]. Failure to 
achieve the national targets resulted in financial, reputational and other sanctions for DHBs, and PHOs and their providers.

Researchers who have investigated the impact of national targets on organisational performance found that DHBs focus on the performance of their hospitals and on those publicly reported national targets at the expense of the other parts of the system that are difficult to quantify and lack the national policy focus [87-90].

According to O'Flynn [80], NPM practices perpetuate a mechanistic view of health systems, breaking it into parts and performance managing each part individually to improve service delivery. The dominant use of contracts and competition has led to fragmentation of planning and delivery of health care and transactional relationships with agents in the system [80].

\subsection{Indigenous health}

Māori are the indigenous population of New Zealand. The British Crown and Māori rangatira (chiefs) signed Te Tiriti o Waitangi to live together under a common set of laws and agreements [91]. Under Te Tiriti o Waitangi (Te Tiriti), the $\mathrm{MoH}$, as the steward of the health and disability system, has responsibility to work together with Iwi, hapū, whānau and Māori to plan, develop, and deliver health and disability services to ensure Māori receive equitable health care and have equitable health outcomes as Pākehā (non-Māori) while protecting Māori cultural concepts, values and practices $[91,92]$.

Cultural identity for Māori is strongly connected with ancestors and the natural environment through whakapapa, which along with whānau and whenua are the foundation of Māori society $[93,94]$. Māori wellbeing has a holistic view that consists of four dimensions: taha tinana (physical health); taha wairua (spiritual health); taha whānau (family health); and taha hinengaro (mental health) [94]. The whakapapa, whānau and whenua along with a balance across the four dimensions are important for overall wellbeing and self and group identity of Māori [93].

According to Smith [95] and Cram, et al. [93], the health care reforms of the 1990s, especially the introduction of a competitive health care delivery model, disrupted whakapapa and severely marginalised the Māori view of wellbeing. Māori holistic view of health care delivery that centred around Iwi, hapū, whānau, relationships, reciprocity and sharing was replaced by Pākehā way of thinking that included individual, user-pay, consumer voice, accountability and competition [95]. According to Māori, the current health system, based on Western views of health and dominated 
by an emphasis on disease, does not meet their needs; whānau are expected to conform to the system rather than the system being responsive to whānau needs [96].

Scholars of Māori health assert that the colonisation process bestowed superior power and access to resources and knowledge to Pākehā that continues today $[93,97,98]$. The loss of land and culture through the colonisation process has resulted in Māori "holding and responding to feelings of injustice, powerlessness and frustration" ( $p$ 7) that continues to impact their health and wellbeing [93].

Health outcomes for Māori are disproportionally poor when compared to non-Māori non-Pacific people, across most health indicators $[96,99]$. Māori health expectancy is lower by at least seven years compared to non-Māori non-Pacific, albeit the gap has narrowed from 9.1 years in 199597 to 7.1 years in 2012-2014 [100]. Māori are more likely to live in areas of high socio-economic deprivation [96].

The structures, policies and practices of a Pākehā-dominated health system means that Māori are less likely to receive optimal health care: for example, immunisation rate is $15 \%$ lower for Māori children; Māori children with asthma are less likely to receive preventive medication; and adult Māori are more likely to wait longer than three months for specialist appointment and are less likely to be prescribed medication to lower cardiovascular risk $[96,97,99]$.

The relationship between Māori and the Crown continue to be challenging. Māori perceive that Te Tiriti is seen as a mere obligation to which the health system leaders respond with tokenism $[96,97,101]$. According to Came, et al. [97], the silence in relation to a commitment to Te Tiriti in national policy and operational documents, a lack of monitoring and reporting of Māori health outcomes, and a lack of recognition of and addressing institutional racism have significantly contributed to the persistent health inequities for Māori.

\subsubsection{The Wai 2575 Health Services and Outcomes Kaupapa Inquiry}

The Waitangi Tribunal is a permanent commission of inquiry set up by the New Zealand government to hear and make recommendations on claims brought by Māori relating to Crown actions that breach the Te Tiriti [102]. In 2018, the Tribunal began stage one of the Wai 2575 Health Services and Outcomes Kaupapa Inquiry in response to claims brought by Māori PHOs and providers. The claimants alleged that the Crown's legislative and policy framework for the primary health care system had failed to achieve Māori health equity and was not fit for purpose. 
The stage one report [101], released in July 2019, reported that the legislative, strategy and policy framework of the sector had breached Te Tiriti commitments in providing equitable health care to Māori. The Inquiry found that the current health system funding arrangements disadvantaged PHOs serving Māori and that the Crown did not adequately amend these arrangements despite being aware of this inequality. A lack of capacity and capability and accountability mechanisms that used the 'golden thread' approach hindered the health system to systematically measure, report and improve Māori health outcomes.

Furthermore, the Inquiry concluded that the primary health care system is not co-designed with Māori. This design failure was reflected both at the national level, within the $\mathrm{MoH}$ with the disestablishment of the Māori health unit in 2015, and at the district level with DHBs lacking true partnership with their governance arrangements to reflect interests of Māori and their local population. The Inquiry found that Māori were significantly underrepresented across the health professions and in the design and delivery of health services.

Overall, the Inquiry found that the strategic, policy and operational documents guiding the health system did not articulate the principles of Te Tiriti accurately at best and at worst did not reference them at all.

The Wai 2575 report recommended five principles of Te Tiriti be adopted by the Crown in the delivery of primary health care:

a) Māori self-determination in the design, delivery and monitoring of primary health care through the guarantee of tino rangatiratanga.

b) A Crown commitment to achieving equitable health outcomes for Māori through the principle of equity.

c) The Crown to achieve equitable health outcomes for Māori through active protection of Māori cultural concepts, values and practices.

d) The Crown to adequately resource kaupapa Māori primary health services and to ensure that all primary health care services are provided in a culturally appropriate way that recognises and supports the expression of hauora Māori models of care.

e) The Crown and Māori to work in partnership in the governance, design, delivery and monitoring of the primary health care system.

Whakamaua, the recently released Māori Health Action Plan, [103] outlines the implementation plan to achieve the overall aim of He Korowai Oranga: whānau ora - "Māori families supported 
to achieve their maximum health and wellbeing" (p 1) [92]. Whakamaua is underpinned by the five principles of Te Tiriti recommended by the Wai 2575 report. The $\mathrm{MoH}$ has committed to measuring and reporting progress against the implementation of the plan.

As a foundation document, Te Tiriti is an important context for New Zealand health system transformation. To meet Māori aspirations and to increase the understanding of health system leaders and staff, the programme architecture that underpins efforts to successfully implement LST initiatives needs to highlight the principles of Te Tiriti and include practical actions to give effect to those principles.

\subsection{Judgements on the quality of care delivered in the health system}

New Zealand's total health expenditure as percentage of GDP is 9.3 percent, which is close to the OECD average [104]. Nominal expenditure has grown from about $\$ 15$ billion in 2014/15 financial year to a budgeted expenditure of about $\$ 20$ billion in 2019/20 financial year [105]. The health system is predominantly funded from general tax, making up 21 percent of total Crown spending in the $2018 / 19$ financial year $[106,107]$.

There is ongoing debate about whether the New Zealand health system is adequately funded [108]; however, the Commonwealth Fund ranking of eleven countries for performance and health outcomes suggests that the level of funding does not always correlate with better system performance and health outcomes.

The Commonwealth Fund compared the health care system performance of 11 high-income countries on measures of care process, access, administrative efficiency, equity and health care outcomes [109]. The United Kingdom's National Health Service had the overall highest ranking of performance with the second lowest spend and received superior performance in care process, access, administrative efficiency and equity. The United States, on the other hand, had the lowest overall ranking of performance with the highest health expenditure and worst ranking for access, equity and health care outcomes. New Zealand was ranked in the top three for care process and administrative efficiency but was ranked below the 11-country average for access, equity and health care outcomes. This ranking reflects the persistent health inequities and poor health outcomes for Māori and Pacific people [110].

The New Zealand Burden of Diseases study shows that New Zealanders' health is improving, with recent increases in life and health expectancy [18]. However, New Zealand still has high health loss from coronary heart disease, chronic obstructive pulmonary disease, chronic kidney disease, 
bowel cancer, self-harm and musculoskeletal disorders. Health inequities persist between genders, generations, ethnic and socio-economic groups $[18,111]$. Health conditions that are both preventable and amenable to timely medical interventions through equitable access to health services make a significant contribution to the lower life expectancy for Māori and Pacific populations. Walsh and Gray [112] found that nearly half of all deaths in Pacific (47.3\%) and over half of all deaths in Māori (53\%) had an avoidable cause compared to under a quarter of deaths (23.2\%) among non-Māori non-Pacific populations.

Results from national patient experience surveys administered by DHBs and PHOs show that the current health system works for most New Zealanders, with patients reporting being treated with kindness and respect and acceptable general practice wait times. However, patients also consistently report issues around communication about medication, co-ordination of care across the health sector (in particular, around the flow of information between hospitals and primary care) and a lack of patient involvement in their own care. People diagnosed with mental health conditions report worse experience of care across the system and Māori and Pacific patients report worse experiences in relation to co-ordination and cost barriers. About 29 percent of Māori and Pacific patients reported not visiting a general practitioner or nurse in the previous 12 months owing to cost in comparison to 19 percent of European patients. The difference by ethnicity for those finding prescription cost to be a barrier was worse, with 24 percent of Māori, 22 percent of Pacific and 7.3 percent of Europeans reporting cost as a barrier [113].

Similar findings are highlighted in the annual New Zealand Health Survey. Results from the survey from July 2018 to June 2019 report that Māori and Pacific adults and children were twice as likely not to have collected a prescription for reasons of cost than non-Māori and non-Pacific adults and children; adults living in the most socio-economically deprived areas were three and half times more likely to be smokers and twice as likely to experience psychological distress; that 13 percent of adults did not visit their general practitioner owing to cost; and children living in most socio-economically deprived areas were significantly more likely to be obese when compared to children living in least deprived areas [114].

There have been national policies and directions to promote changes and improvement in the system, with a desire for the health services to be patient-centred, high quality, co-ordinated, integrated and equitable. This has included the introduction of national initiatives such as the New Zealand Triple Aim [16], the direction for DHBs and PHOs to form Alliances to enhance integration of hospital and primary care [2], and national strategies such as the New Zealand 
Health Strategy [115], the Primary Health Care Strategy [116], and He Korowai Oranga and Whakamaua (Māori Health Strategies) [92]. Some scholars called for a fourth dimension relating to health care workforce to be added to the Triple Aim framework [36]. However, the Institute of Healthcare Improvement and the New Zealand Health Quality and Safety Commission retained the use of the original Triple Aim framework to guide systemic improvement initiatives to ensure delivery of equitable and high-quality health care $[16,36]$.

Some progress has been made with these initiatives but implementation, monitoring and ongoing endorsement from the government, including from the $\mathrm{MoH}$ and $\mathrm{DHBs}$ have been variable. Further, the series of policy initiatives to improve performance have not been coherent. In reality, this has meant that the government continued to use NPM ideas to focus on DHBs' performance using financial and hospital performance measures while simultaneously exploring ways to improve the performance of the system.

The financial performance of DHBs varies and has deteriorated over the last five years driven mostly by growth in personnel costs, rising demand for health services, a lack of long-term system transformation initiatives, and governance and management challenges [117]. For the financial year ending June 2019, the 20 DHBs reported a total deficit of just over $\$ 1$ billion [117]. Both the Minister of Health and the MoH are working with DHBs to improve their financial performance. For three of the DHBs, which posted the largest deficits, the Minister of Health has appointed a Commissioner along with a Crown Monitor for one and Crown Monitors for the other two to establish recovery plans [117]. The MoH's focus is to closely monitor the financial performance of DHBs with deficits with the aim to improve current financial performance [117].

\subsection{The Health and Disability System Review and the upcoming reform}

In the endless pursuit to improve performance of the New Zealand health system, the centre-left government elected in 2017 commissioned a major review of the New Zealand health and disability system [118]. The purpose of the review was to investigate if the health system was meeting its legislative obligations of providing effective and equitable health outcomes for all New Zealanders and if the system was strong and sustainable. The review led by an expert panel, was tasked with making recommendations on changes needed in the system so it would be better placed to cope with future demands and be balanced towards wellness, access, equity and sustainability. The final report of the Health and Disability System Review released in June 2020 made 86 recommendations for the New Zealand health system to achieve equitable health outcomes and to become a more financially sustainable system [23]. 
The recommendations include significant organisational reforms, reducing the number of DHBs from 20 to between eight and twelve and forming two-tier services: tier one includes primary and community; and tier two includes hospital and specialist services. DHBs would no longer be required to contract primary care services through PHOs or form Alliances with them. Instead, the review recommended that DHBs use locality networks to plan and deliver tier one services and use these networks to integrate tier one and two services.

The review recommended establishment of two new national agencies: Health New Zealand and Māori Health Authority. These, along with a smaller, more policy focused MoH, would have direct accountability for the system's performance to the Minister of Health. Health New Zealand would oversee the operational and financial performance of DHBs, improve co-ordination of quality initiatives with clinical engagement, and reduce variation in performance between DHBs by coordinating the delivery of complex highly specialised services. This would see a separation of the national service planning, funding and delivery function from the national policy leadership role. The latter will be retained by the $\mathrm{MoH}$, which remains the chief steward of the health system. The Māori Health Authority would advise the government on all aspects of Māori health policy, monitor and report on Māori health outcomes, partner with other organisations at all levels to integrate Te Tiriti o Waitangi principles into policy, planning and service delivery, and strengthen the Māori workforce.

Along with organisational reforms, the review also recommended development of a national charter that outlines the shared values of the system (Box 2.2) and a new health plan that guides the long term (10 to 15 years) strategic direction of the system, supported by short-term regional and district plans. The review briefly mentions the importance of increasing national system leadership capability to create the conditions for leaders within the system to develop and work effectively. There is a focus on increasing DHB governance capability with DHB board members no longer elected but appointed by the Minister of Health using a competency-based approach.

Finally, the review identified three enablers required to produce effective health outcomes and to ensure the system is financially and clinically sustainable. These are workforce, data and digital technology, and facilities and equipment. 


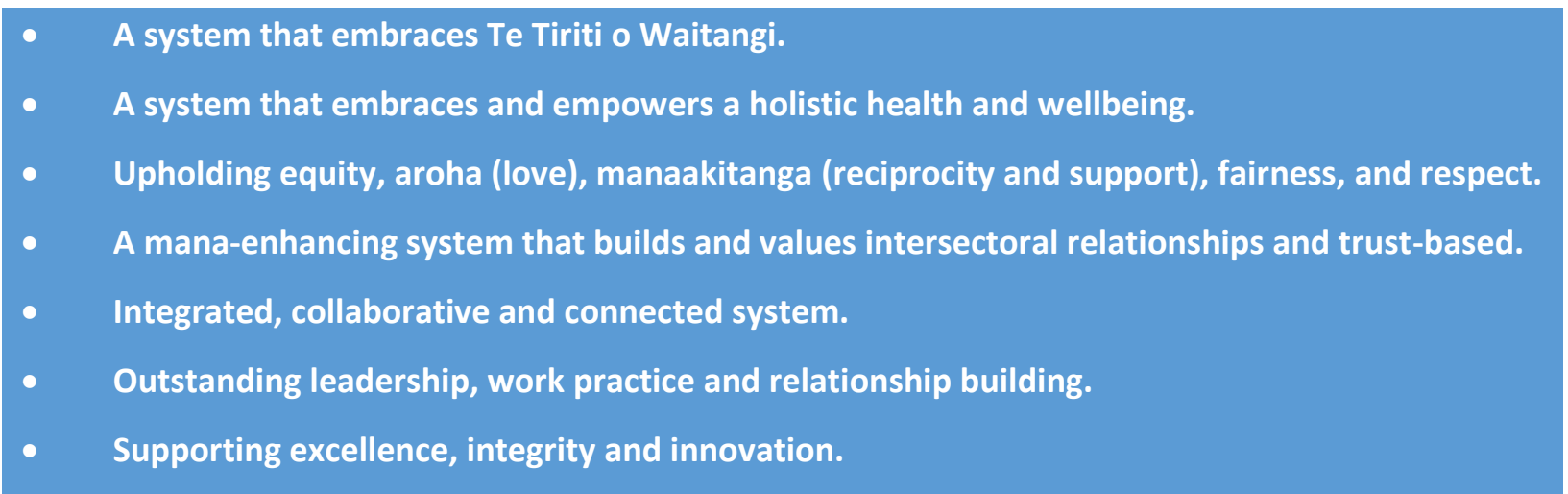

Box 2.2: Proposed shared system values [23]

The review did not recommend any particular funding model or levels of funding because it did not consider funding to be the sole cause of health inequities or DHB deficits. It did, however, recommend changes in legislation to allow for a guaranteed annual increase in health funding to continuously reflect changes in the population demographics, that ethnicity weighting is applied to all population-based funding formulas with at least 20 percent weighting for Māori and Pacific populations for tier one services, and that funding for tier one services (primary and community) is ring-fenced.

While the interim report of the Health and Disability System Review acknowledged that leadership and culture change was needed and that the system needed to work collaboratively, the final report focused on significant organisational reforms relating to planning and funding functions at a macro-level and ways to enhance accountability in the system akin to an NPM approach $[23,96]$. The review did not consider the successes and the challenges of the SLM programme nor the role of Alliances in implementing such transformation initiatives. The upcoming reform is likely to disrupt the SLM programme, in particular the use of Alliances to implement the programme. 


\subsection{Chapter summary}

Despite three decades of policy initiatives to improve integration of health care, scholars and reviewers regularly conclude that the delivery of health care remains fragmented and focused on institutional arrangements $[21,22,24,96]$. The governance and institutional arrangements that separate service delivery of hospital, primary and community services, and the interests of powerful professional groups, have led to each service or group looking after their own interests and those of the patients in their service or speciality; and not the broader interests of patients and the population perspective $[54,96,119]$.

Reforms during the 1980s and 1990s focused on planning and funding functions at a macro-level and did not bring about service integration at meso- and micro-level to deliver co-ordinated patient-centred care and reduce health inequities [21]. Furthermore, the 1990s reforms were based on market-oriented government ideology and NPM ideas, with limited evidence to suggest that they would achieve their goals, came at huge costs, were driven from the top, and disengaged health care professionals $[56,60]$. There has also been little or no focus on governance models and health system leadership capacity and capability, and leadership in the system is lacking at all levels $[21,96]$. The upcoming reforms continue the theme of previous reforms of focusing on planning and funding functions at a macro-level, centring on organisational restructure, and are being driven from the top.

Governance models such as Alliances have encouraged more collaboration between DHBs and PHOs to improve health outcomes by sharing health information and resources to deliver integrated and co-ordinated care that is best for their population [68]. These models are underpinned by 'new power' values such as a high-level of trust among members and strong relationships with members committing to shared responsibility, shared decision-making, and shared accountability [68]. Governance models such as Alliances have the potential to reduce fragmentation in the delivery of health care and bring organisations together to successfully implement LST initiatives and adopt and sustain innovations at a system level. However, more effort is needed to understand the inner workings of these networks and it is important to share these insights with health system leaders and with the implementers of the Health and Disability System Review. 
The relationship between Māori and the Crown remains challenging and any LST initiative needs to meet aspirations for Māori. While the New Zealand health system may work for most, disconnected services and lack of co-ordinated care between different parts of the health system contributes to poor health outcomes, particularly for Māori and other high priority populations, and leads to inefficient use of scarce health resources. In order to achieve effective, equitable, efficient, high-quality and sustainable delivery of health care, a simultaneous focus on all three dimensions of the Triple Aim, at a system level, is required [36]. LST initiatives, such as the SLM programme, aim to pursue this simultaneous focus.

The next chapter describes the development and implementation of the SLM programme: an initiative designed to achieve system transformation through a collaborative way of working using Alliances. 


\subsection{THE NEW ZEALAND SYSTEM LEVEL MEASURES PROGRAMME}

The SLM programme was developed by the $\mathrm{MoH}$, together with the DHBs and PHOs, and is a LST initiative designed to support health system improvement and integration [15]. The programme was implemented in July 2016 and continues at the time of writing this thesis. The key purpose of the programme is to enhance a collaborative way of working beyond organisational and professional boundaries, address health inequities and encourage continuous learning and quality improvement [15]. The key purpose of the programme is to achieve the Triple Aim.

\subsection{Development of the programme}

The journey of the SLM programme began in 2012 with the General Practice Leaders' Forum's (GPLF) desire to develop a more meaningful performance improvement programme for primary care in New Zealand. The GPLF proposed to transform the PHO Performance Programme (PPP) and the incentive funding attached to this programme. The GPLF and the MoH commissioned a report through an expert advisory panel. In their report to the MoH [39], the Expert Advisory Group outlined their vision for an Integrated Performance and Incentive Framework (IPIF) that addressed equity, safety, quality and cost of health services; shifted the focus from outputs to outcomes; encouraged collaboration between health providers; and created an environment for local, clinically-led continuous quality improvement. IPIF had a primary care focus, with the intent over time to broaden and include other community providers and (eventually) hospital care.

The $\mathrm{MoH}$ began to co-develop a set of system-level measures based on a life course approach (from conception, infancy, childhood, adolescence, adulthood and older age) with measure development groups. Initially, there were two measure development groups; healthy child and healthy ageing. These groups comprised clinicians, managers, academics and patient advocate groups. Additionally, there was an analytic technical advisory group. Measures were proposed by the measure development groups and then assessed for feasibility of implementation by the analytic group.

A change within government in 2015 (a change of Minister of Health) meant a change in the direction, and development of IPIF was paused. The renewed focus of the work aligned with a whole-of-system approach, which engaged the health sector more broadly (beyond primary care) than IPIF.

The life course approach was retired, and instead the $\mathrm{MoH}$ was directed to develop a small set of about six to eight health system outcome measures that focused on children and youth, value 
for resources, patient experience, and prevention and early detection. A new measure development group was formed, which considered over 100 existing measures to identify those that were focused on health outcomes, were meaningful to clinicians and consumers, where data was reliable and available in a timely manner, were able to be reported by ethnicity and social deprivation index, and, where possible, used data that was already collected and reported so it did not increase the reporting burden on the sector.

Between July 2015 and June 2016, using an iterative consultation process with the broader sector, the group produced a short list of twelve measures from which the Minister of Health agreed on six to be implemented under the new programme of System Level Measures Framework (the SLM programme).

The six nationally agreed system-level-measures (SLMs) are: Ambulatory Sensitive Hospitalisation (ASH) rates for zero to four-year olds; acute hospital bed days per capita; patient experience of care combining adult hospital and primary care patient experience surveys; amenable mortality rates; babies living in smokefree homes; and youth access to and utilisation of youth appropriate health services [15].

The SLMs aligned with the New Zealand Triple Aim and were deliberately chosen so that no one single provider in the health and disability system could sincerely (without gaming) improve the measure, no matter how efficient and high performing it was [40]. Instead, the aim was that improvement would be achieved by all agents in the system working together using improvement science with a 'bottom-up' approach that involved frontline clinicians, service users and local communities. The idea was to encourage locally led quality improvement actions that addressed local population health needs and that linked to the national system improvement direction through a small set of health outcome measures. The six SLMs addressed ageing, chronic disease, and equity issues present in the New Zealand health system. They measured hospital use by children, youth, and people over 65 years of age as a result of health conditions that could be prevented and managed better in primary care to avoid hospitalisations with DHBs and PHOs working together in the best interest of their population.

The SLMs are supported by a suite of process and activity measures, known as the contributory measures, all of which are available through an online measures' library [15]. The measures' library contains technical information about SLMs and contributory measures, such as, measure definitions, data source and the method for calculating the measure. The library was created so 
implementers could easily see the logic of how SLMs are connected to contributory measures and that all measures used in the programme are nationally comparable. Alliances are expected to use SLMs to determine the focus of improvement for their districts (improvement milestones), determine local actions to achieve their improvement milestones, and then choose relevant contributory measures to monitor progress against their improvement actions [15].

Incentive funding that was attached to the PPP was re-negotiated for the SLM programme. Seventy-five percent is paid to PHOs to develop capacity and capability for development and successful implementation of an annual SLM plan and 25 percent is paid on achieving the milestones for three SLMs and two National Health Targets: increased immunisations; and better help for smokers to quit [63].

The concept of using whole-of-system measures to measure the quality of the health system has been implemented in several health care systems internationally; for example in Sweden, Wales and the United States [120]. According to Chalmers, et al. [41], the New Zealand SLM programme is unique in two ways. First, it attempts to steer a middle course between the extremes of traditional health targets and pay-for-performance policy tools that focus on single organisations, and broad population health outcomes that are influenced by wider social sector services and are beyond the sole influence of health systems. Second, the emphasis is on a collaborative way of working using Alliances rather than financial and reputational carrots-and-sticks approach. The authors acknowledged that no other country "has attempted to implement such an 'Alliancebased' approach to health system improvement at a national level" (p 4) [41].

The co-design of SLMs with the health sector, local clinically led continuous learning and quality improvement, and a collaborative way of working through Alliances to create a collective sense of purpose formed important underpinnings of the SLM programme philosophy [40].

\subsection{Implementation of the SLM programme}

Implementation of the SLM programme required DHBs and PHOs to work together, share health information and resources, identify priorities for their district, and agree an integrated plan and commit to delivering it. At the end of year, they were expected to review the implementation of the plan using a PDSA cycle, reflect on their successes and failures and incorporate lessons in the following year's plan.

In order to bring system leaders together and build new collective forms of accountability, the $\mathrm{MoH}$ placed responsibility for leading the implementation of the SLM programme with Alliances 
(discussed in Chapter 2, section 2.3). Alliance leadership teams are tasked with development of an annual improvement plan using a collaborative approach underpinned by robust improvement science, and monitoring and reporting progress against successive plans [15].

Since Alliances are not legal entities, accountability for the SLM programme sits with DHBs. Accountability is embedded through the DHB Operational Policy Framework (OPF) [2] and the SLM plan is part of the DHB annual plan.

The improvement plan includes improvement milestones for each of the six system level measures; the frontline improvement actions; the contributory measures; and the signature of all Alliance partners. The plans are submitted to the MoH by DHBs on behalf of their Alliances. The signatures on the plan are a proxy designed to demonstrate an integrated and partnership approach to the development and implementation of the improvement plan. Figure 3.1 shows the process Alliances are expected to use for the development of the plan; using ASH rates for zero to four-year olds system level measure as an example [40].

The plans are assessed and approved by the $\mathrm{MoH}$ and quarterly reports from Alliances, submitted by their DHBs, demonstrate the progress against the plan. At the end of the planning year, Alliances report whether they implemented the plan and achieved their improvement milestones for the six system level measures; and if not, why not, using a plan-do-study-act process and sharing their insights and learning to inform the following year's plan. Figure 3.2 shows the intervention logic for the SLM programme.

The SLM programme mobilised hundreds of people in the health system to improve the health of their populations using a collaborative approach [15]. Implementation of the programme included health care professionals, health system leaders and consumer councils in DHBs and PHOs. In some districts implementation also included other health system partners such as pharmacy, Māori and Pacific providers, youth health service providers and the ACC.

The collaborative design of the programme and implementation through Alliances recognised that successful implementation would require high-trust relationships with a mix of national and local accountability mechanisms between the $\mathrm{MoH}, \mathrm{DHBs}$, and Alliances. At the local level, implementation would require high-trust relationships among the Alliance members, a commitment from system leaders to implement the plan, adequate information infrastructure to inform the local improvement actions, and a continuous improvement approach to maximise successful implementation. 
- Use local district data available to identify the percentage of potentially avoidable hospital admissions for children aged 0-4 years in your alliance or

Analyse your data and

identify main

contributors to ASH service level alliance teams

- Break down by ethnicity and deprivation level to identify equity gaps

- Look at most common conditions in children: respiratory illness, dental conditions, gastroenteritis and skin infections

Identify improvement milestone

Identify improvement actions and providers that will impact the milestone

Select most relevant contributory measures
ASH improvement milestone: ASH rates for Māori and Pacific children fall by $2 \%$ (432) by end of June 2021
To achieve the milestone with focus on Māori and Pacific children and families:

- Introduce healthy homes initiative through NGO and Public Health Unit

- Launch smoke-free homes campaign, focusing on Māori and Pacific families

- Comprehensive diagnosis and treatment of asthma in primary and community care including general practice, pharmacies and ambulance

See the ASH contributory measures on the Health Quality Measures Library website and include most relevant ones:

- Number of healthy homes referral

- Six-week-old babies living in smoke-free homes

- Four-year-old children living in smoke-free homes

- Hospital admissions for children aged five years with a primary diagnosis of asthma

\begin{tabular}{|c|c|c|c|}
\hline \multirow{5}{*}{$\begin{array}{l}\text { Develop and submit } \\
\text { improvement plan to } \\
\text { MoH with ALT signatures }\end{array}$} & \multicolumn{3}{|c|}{ ASH rates for 0-4-year olds } \\
\hline & $\begin{array}{l}\text { Improvement } \\
\text { milestone }\end{array}$ & Improvement actions & Contributory measures \\
\hline & \multirow{3}{*}{$\begin{array}{l}\text { ASH rates for } \\
\text { Māori and } \\
\text { Pacific children } \\
\text { fall by } 2 \% \text { ( } 432 \text { ) } \\
\text { by end of June } \\
2021\end{array}$} & $\begin{array}{l}\text { Introduce healthy homes } \\
\text { initiative through NGO and Public } \\
\text { Health Unit }\end{array}$ & $\begin{array}{l}\text { Number of healthy homes } \\
\text { referral }\end{array}$ \\
\hline & & $\begin{array}{l}\text { Launch smoke-free homes } \\
\text { campaign, focusing on Māori and } \\
\text { Pacific families }\end{array}$ & $\begin{array}{l}\text { Six-week-old babies living in } \\
\text { smoke-free homes } \\
\text { Four-year-old children living } \\
\text { in smoke-free homes }\end{array}$ \\
\hline & & $\begin{array}{l}\text { Comprehensive diagnosis and } \\
\text { treatment of asthma in primary } \\
\text { and community care including } \\
\text { general practice, pharmacies and } \\
\text { ambulance }\end{array}$ & $\begin{array}{l}\text { Hospital admissions for } \\
\text { children aged five years with } \\
\text { a primary diagnosis of asthma }\end{array}$ \\
\hline
\end{tabular}

Monitor and report progress on implementation of the plan to DHB and $\mathrm{MoH}$. Use insights in development of the following year's plan.

Figure 3.1: Improvement plan development process 
Problem statement:

Disconnected services and lack of coordinated care between different parts of the health system contributes to poor health outcomes, particularly for Māori and other high priority populations, and inefficient use of health resources.

To provide a framework that enhances system integration,

eliminates health inequities, and drives continuous quality

improvement using 'bottom-up' approach to improve delivery of

integrated and patient-centred care.

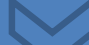

Underpinning theory based on evidence:

The demands of an evergrowing aging

increasing prevalence of long-

term conditions and a

constrained fiscal environment have prompted a review of

how health and wellness

services are provided.

Evidence internationally

supports that well integrated

care organised under the

Institute of Healthcare

Improvement's (IHI) Triple Aim

improves value for money,

population health and patient

experience. The IHI supports

the use of quality indicators to

show performance of the

health system over time in

achieving the Triple Aim.

SLM programme encompasses

a new way of working and

different philosophical

approaches such as a whole-of-

system focus, collaboration and

co-production with the sector

harnessing the knowledge and

influence of sector leaders, and

building high trust and

sustainable relationships both

at national and district levels.

\section{Resources/inputs}

People - clinicians, managers, analysts, consumers, quality improvement advisors, project managers

Time - for everyone to participate Information technology to enable storing, analysing and sharing of information

Data - both identifiable and aggregate levels, real-time and time trend, by ethnicity, gender, conditions, age, socio-economic status, administrative etc

Formal agreements between health providers to share data Partnership forums such alliance leadership team, clinical governance groups, service leve alliances, consumer councils Shared goals and commitment to placing patients and health equity at the centre of service design and delivery; learning and doing things differently Shared performance management and accountability framework i.e. reporting at alliance level, shared decisionmaking framework

Quality improvement tools Funding

\section{Longer term impacts (conditions)}

No health inequities for Māori and other high priority populations Timely care in secondary services

Prevention, early detection and good care in the community Improved system leadership capacity and capability earning system based on constant feedback loops Patients experience integrated care

\section{Activities - what will be done by}

\section{district alliances?}

Establishing a functional alliance, project teams and clinical champions for SLMs

Use data and evidence to understand local population demographics and needs Identifying equity gaps for Māori and other high priority groups

Use of patient experience surveys to hear patient voice Identifying areas of improvement for each SLM

Aligning work programmes and initiatives within and across providers

Agreeing on quality improvement initiatives to be undertaken by each provider

Choosing appropriate contributory measures to measure local progress

Developing the SLM plan Seeking agreement of all parties to the improvement plan Implementing the approved improvement plan

Regular monitoring and reporting at alliance level and to $\mathrm{MoH}$ Continuous quality improvement PDSA cycles for continuous learning

\section{Outputs}

District alliance leadership teams

Measures of outputs

SLM plan that identifies:

mprovement milestones for eac

SLM

Frontline actions to achieve the improvement milestones, with a

focus on addressing equity gaps

Contributory measures that will

measure local progress against the actions and provide line of sight to the SLMs

Commitment from all parties to the delivery of the improvement plan

Project plan that identifies:

Resources and funding to deliver on the initiatives and activities identified in the SLM plan

Local reporting and accountability processes for all parties to the SLM

Successful implementation of the SLM plan

Regular reports to alliances against the improvement plan

\section{Quarterly reports against the}

improvement plan to $\mathrm{MoH}$ showing

incremental improvements over time

\section{Medium term impacts (action)}

Reducing equity gaps for Māori and other high priority groups Continuous improvement culture

Improved integrated health information and analytic capability Improved commissioning skills

Trust-based relationships

Strong clinical leadership

Strong partnership with people, whānau, Iwi and communities

Short-term impacts (learning)

Mature and functional alliances that involve all parts of the health system

Alliances using constant feedback loops for continuous

improvement

Use of data and evidence to reach and respond to individuals change in service delivery models

Sharing of good practice and scaling successful initiatives Increased use of continuous quality improvement science Improved data availability and reliability

Achievement of the SLM milestones

\section{External factors that may affect impacts}

DHB financial position

DHB and PHO relationship and leadership

$\mathrm{MoH}$ relationship and leadership and support for sector

Impact of wider social determinants of health that is outside the influence of the health sector eg housing, income,

employment etc

Change in Government priorities

Central policies and funding arrangements

Assumptions:

Framework relies on intrinsic motivation of health providers to work together to improve population health outcomes.

Framework relies on districts having a functional alliance.

Programme implementation assumes a high trust operating environment.

Framework relies on health funders' willingness to change models of care and funding arrangements 


\subsection{My role in the SLM programme}

I have spent most of my working life in the New Zealand health system: starting as a Health Protection Officer in 2000 responsible for the delivery of health protection services; and later as a senior leader responsible for managing the delivery of population-based public health services. For the last five years I have been working at the $\mathrm{MoH}$ leading the SLM programme.

Having been involved in providing advice to government to set up the SLM programme, which included agreement with the Minister of Health on the six measures and the allocation of the $\mathrm{PHO}$ incentive funding, the $\mathrm{MoH}$ was then responsible for overseeing its implementation. The MoH's role in the implementation of the SLM programme included: supporting Alliances to develop their annual SLM plans, approval of these plans, and monitoring Alliance progress against these plans. The $\mathrm{MoH}$ facilitated relationships among primary, community and secondary care services, in particular between DHBs and PHOs, and engaged clinicians in the implementation of the SLM programme. The $\mathrm{MoH}$ also managed the $\mathrm{PHO}$ incentive funding.

As the programme progressed, the role of the $\mathrm{MoH}$ extended to the implementation of the national primary care patient experience survey with the Health Quality and Safety Commission; and the sharing of identifiable level data with DHBs and PHOs from national collections to support quality improvement initiatives.

The SLM programme clinical lead and I undertook most of the $\mathrm{MoH}$ functions described above. At the onset of the implementation, both the clinical lead and I realised the important but challenging task we had with the implementation of this programme compounded by a short lead-in time. We agreed that it was important for us to demonstrate the philosophies of a collaborative way of working and a continuous improvement approach both to the way the programme was implemented and to the programme outcomes. We took a long-term view of the programme and our approach was that as the programme matured and the processes became embedded, we would expect an incremental increase in the quality of improvement plans and achievement towards the programme medium- and long-term outcomes (Figure 3.2).

We were cognisant of external factors that may impact on the successful implementation of the programme: DHB financial performance; the $\mathrm{MoH}, \mathrm{DHB}$ and $\mathrm{PHO}$ relationships and leadership styles; the impact of the wider social determinants of health; and changes in government priorities, national policies and funding arrangements. We relied on the intrinsic motivation of health care staff to work collaboratively to improve health outcomes for their populations, a high 
trust operating environment, health system leaders' willingness to change models of care and funding arrangements, and most importantly, we relied on districts having a functional Alliance. We thought that the implementation of the SLM programme would give Alliances a collective purpose leading to broadening of the Alliance membership and strengthening the alliancing way of working.

In the first year of implementation, our aim was to receive a plan from each of the Alliances regardless of the quality of the plan. This recognised that the programme required clinical and operational leaders from across the system working collaboratively with frontline clinicians to implement a quality improvement programme in a way that had not been done before. Alliances could choose to maintain their performance in the first year while they examined their data to understand what was driving their rates for the system level measures. The Alliance also did not have to provide their improvement actions in the national plan. These were expected to be part of their local project plans.

In the second year, we raised our expectations and asked that Alliances seek an improvement from their past performance for the system level measures and include a brief description of improvement actions in the plan submitted to the $\mathrm{MoH}$.

In the third year, we emphasised the need for the plans to focus on addressing health inequities. This required involvement of Māori and Pacific teams in DHBs and a stronger line of sight between the milestones, actions and the contributory measures. We also asked Alliances to extend their membership beyond DHBs and PHOs and include other partners in the system such as patients, communities and Iwi, ambulance, pharmacy, and maternity.

Along the way, we made changes to the programme based on feedback received from Alliances and frontline staff involved in the implementation of the programme. For example, initially, there was a requirement for Alliances to set an annual improvement milestone for all six measures, however feedback from sector led to change for one of the measures (amenable mortality rates), for which Alliances were required to set a three-to-five-year milestone. We also co-developed a new system level measure for youth health with the sector and the young people.

My role in the development and implementation of the SLM programme included thought leadership as well as these activities listed: developing national annual guidance for implementation of the programme; supporting Alliances to understand the philosophy of the 
programme and develop local improvement plans; approving annual SLM plans; assessing Alliances' progress against their plans; and connecting with other teams in the $\mathrm{MoH}$.

The clinical lead and I would attend local planning workshops and meetings to support DHBs and their Alliances in the early stages of development of the plan. We visited all Alliances in New Zealand and at times facilitated relationships among senior leaders from health service providers, in particular, relationships between senior clinical and operational leaders from DHBs and PHOs. We also attended clinical leadership forums, SLM steering and working groups, PHO Chief Executives' meetings, DHB planning and funding managers' meetings, Māori General Managers meetings, and the PHO quality improvement network to seek a whole-of-system engagement in the programme and to emphasise its philosophies.

Each year, as part of approving improvement plans, the clinical lead and I would work with Alliances and other groups involved to ensure that all Alliances had an approved improvement plan and that the plan had improvement actions that would lead to better health outcomes. The approval process would usually take up to four months with an iterative process of assessing and providing feedback on several versions of each plan from the first draft to the final approved version.

The clinical lead and I had the privilege of observing and experiencing some of the conditions that enabled and constrained the implementation of the programme across DHB districts. We gained invaluable insights and knowledge into why some districts were more successful than others in bringing together their local system partners to develop a high-quality plan and fully implement the plan. We also experienced how the prevailing structures, behaviours and processes shaped the $\mathrm{MoH}^{\prime}$ s role and resource allocation for the SLM programme.

In response to local health systems' quest for guidance on how to be more successful with their SLM programme implementation, we started to share some of these insights and knowledge through a number of forums such as meetings, informal conversations and planning guidance documents. Through these interactions, patterns emerged, which stimulated theories about the architecture for successful implementation of the programme. However, such knowledge and insights remained with individuals in the system, such as the system leaders, the frontline staff involved in implementation, the clinical lead and I. It also became apparent that DHBs and PHOs had different understandings of an alliancing way of working, of what constituted an Alliance and of what a functional and mature Alliance looked like. Although there was a national charter for Alliances, this was variably interpreted and used, and the system lacked a tool that Alliances could 
use to assess where they were on a maturity scale or what they needed to do to become better at implementing LST initiatives such as the SLM programme.

The institutional knowledge and insights on the SLM programme have been gained through leading the implementation of the SLM programme across New Zealand. Also, I had opportunities to make presentations at national conferences and at an international conference in Australia, which provided the opportunity to network with international health system representatives. The unique approach with the SLM programme generated significant interests from international health systems, in particular from Australia and Scotland.

\subsection{Evaluation of the SLM programme}

My insights and knowledge suggested that success of the SLM programme required high-trust relationships between system leaders (both operational and clinical), an access to patient health records, a strong analytic capability, flexible commissioning approaches, and an improvement focus to nudge the system to one that is continually learning.

An evaluation of the SLM programme studied the internal and external conditions that contributed to the successful implementation of the programme [121]. The reviewers interviewed about 50 participants from across all DHBs and analysed SLM plans from the first three years of implementation. The study used two success criteria: the maturity of SLM plan processes and the sophistication of information use.

In the first success criterion, the authors reported that high performing districts had collaborative and inclusive planning processes that involved a broad range of stakeholders from primary and secondary care. In the initial implementation stages, the study found that majority of DHBs and PHOs were unclear on the implementation process and that implementation was dominated by either the DHB or the PHO. The study reported that the clarity on the implementation process changed as the programme matured. By the third year of implementation, many districts reported having a 'bottom-up' approach to the development of the plan with gradual widening of clinical and organisational involvement.

The second success criterion assessed the district's capacity and capability to gather, use and interpret data for quality improvement processes. The study found there was mixed success with this criterion with some Alliances having dedicated, funded roles for implementation of the SLM programme, others having ad hoc support from existing roles within the $\mathrm{DHB}$, and some Alliances being supported by larger PHOs. Better performing districts had better capacity and capability 
that included data sharing mechanisms between DHBs and PHOs; access to granular, reliable and accurate data; and analytic capability to translate data into knowledge and actions.

When investigating the conditions for success, the study found that there was a high level of understanding on the aims and logic of the SLM programme that included: a whole-of-system integration; the shift from performance management to quality improvement; addressing health inequities; and the shift from measuring outputs to outcomes. On the other hand, respondents reported challenges in understanding how the SLM programme related to the other planning processes in the district, for example the DHB annual plans or the roll out of the Healthcare Home initiative.

The study examined two internal conditions that influenced the successful implementation of the programme: the maturity of Alliances, and the quality of relationships and the level of trust between DHBs and PHOs. High performing districts reported a history of working together successfully with longstanding commitment to collaboration and an agreed integrated work programme. Conversely, poor performing districts reported a history of challenges in relationships between DHBs and PHOs. Some reported that the introduction of the SLM programme provided opportunities to improve relationships.

Two external conditions were examined: the size of district, and the complexity of interorganisational environment, for example, where the district had multiple PHOs operating, or where there was one PHO across multiple districts. The reviewers wanted to test if more complexity reduced the chance of success and if districts with larger populations had better capacity and capability but also more challenges in developing and sustaining high-trust relationships.

The study found that maturity of SLM plan processes correlated directly with two conditions: a district's understanding of the aims and logic of the SLM programme and having a mature and functional Alliance. The latter had a stable structure with allocated responsibilities, a clear Alliance charter, an inclusive Alliance leadership team that extended beyond DHBs and PHOs, an established integrated work programme for the district, and shared commitment to the alliancing way of working. Districts with less mature SLM plan processes lacked the presence of either one or both conditions. 
For the sophisticated use of data success criterion, the quality of relationships was a key condition. Districts with sophisticated use of data had positive and high-trust relationships between organisations.

The two external conditions - the size of the district and the complexity of inter-organisational environment were found to not influence the successful implementation of the programme. The study found that the two internal conditions - a lack of Alliance maturity and poor informal relationships between organisations - adversely influenced the success of implementation rather than the two external conditions.

The study also reported that the capacity and capability funding available to PHOs created a barrier to engage other health system stakeholders and facilitate integration because of the exclusivity of the funding for the programme being available to PHOs. While many PHOs had used the funding to enhance their quality improvement processes and fund new improvement actions, there were issues around purpose and transparency on how this funding was used.

The study concluded that successful implementation of LST initiatives such as the SLM programme requires a strong platform of an alliancing way of working, positive and high-trust relationships between organisations, and robust improvement processes that enable continuous learning.

Findings of the evaluation closely matched my experience and observations. At the beginning stages of implementation of the SLM programme, I believed the programme facilitated and strengthened an alliancing way of working, relationships between DHBs and PHOs and continuous improvement capacity and capability. My experience, evaluation of the SLM programme, and implementation science and LST literature, found these to be pre-conditions for successful implementation of LST initiatives such as the SLM programme [1,3,30,37,121-123]. This revelation strengthened my motivation to explicitly reveal the programme architecture that underpins efforts to successfully implement LST initiatives, including a practical tool in the form of a self-assessment maturity matrix.

The next chapter reviews international literature to understand the academic context for LST initiatives using a complex adaptive system lens, the role of informal networks such as Alliances in the implementation of LST initiatives and the role of maturity matrices as a quality improvement tool to support the notion of a learning system. 


\subsection{ACADEMIC CONTEXT FOR THE PROPOSED RESEARCH}

This chapter applies complexity thinking to health systems and describes these as complex adaptive systems. Change in complex adaptive systems requires LST initiatives to achieve the desired system transformation. The chapter introduces the concept of 'old power' and 'new power' that influence the successful implementation of LST initiatives. The final section reviews the literature on the use of maturity matrices in health care setting and how this can be used as a tool to support the notion of a learning system.

\subsection{Health as a complex adaptive system}

Delivery of health care has significantly evolved in the $21^{\text {st }}$ century. The focus of the health system in the $19^{\text {th }}$ and $20^{\text {th }}$ centuries was on treating diseases and providing a place for injured soldiers to convalesce [124]. By late $20^{\text {th }}$ century, primary health care was typically provided through privately owned single general practitioner with a small team but the focus remained on acute episodic care with hospital care delivered separately [28]. Health care professionals relied on evidence and knowledge obtained at medical school to diagnose and treat illnesses singly at an individual level [28]. Delivery of health care and leadership of health care organisations were influenced by machine metaphors and Newton's clockwork universe. The central idea to the machine metaphor was that the health system is a mechanical system with defined boundaries, linear functions and in which outputs can be predicted $[28,125]$. Newton's clockwork universe was based on universal laws of motion and gravity in which the whole system can be broken into parts and the behaviour of the whole could be understood, controlled and predicted by studying each part $[28,125]$.

The mechanistic approach was useful in the $19^{\text {th }}$ and $20^{\text {th }}$ centuries with clinical medicine and led to discoveries of antibiotics and analgesics, and advancement in clinical practices such as surgeries [125]. The late $20^{\text {th }}$ century saw a change in demand for and expectations from the health system with a focus on wider social determinants of health. Longer lifespans and the growing burden of long-term chronic conditions with ageing populations required a shift in the health care delivery from acute episodic care by a single practitioner to long-term management of chronic conditions by a multi-disciplinary team [27]. Health care was no longer just delivered by doctors and in hospitals, but by a range of health care professionals in a variety of settings. The health system became complex with many agents that included hospitals, general practitioner teams, aged-residential care homes, allied health teams, community care providers, pharmaceutical companies, and patients and families [126]. The significant pace of advancement 
in technology was increasing health care expenditure and pharmaceuticals were gradually increasing the cost of health care delivery [27]. Expectations from public on the way health care was delivered also changed significantly, away from a provider- and disease-centric to a holistic wellbeing approach that was patient- and family-centric [27].

The change in demand and expectations made apparent the limitations of a mechanistic approach [8]. The enormous complexity of the health systems, and the fragmented approach to managing and delivering health care raised serious issues around the quality of care provided and feedback from patients on the difficulty of navigating the health system [27]. Reviewers of health systems highlighted weaknesses of the siloed approach from hospitals and general practitioners that led to serious adverse clinical outcomes, under and over utilisation of services and unintended consequences with the use of pay-for-performance approaches that led to health providers focusing on one intervention at the expense of others $[27,126]$. The complexity, financial pressure to manage costs and unintended consequences led the policy makers and researchers to seek new ways of understanding the health system. The early $21^{\text {st }}$ century saw an emergence of using complexity theory that originated from the field of quantum physics and focused on relationships, emerging patterns and constant adaptation to changing environments. Over the last two decades, complexity theory has been used to frame health system as an ecosystem that is complex, living and adaptive and in which relationships between agents in the system are more important than the efficiency of each agent $[7,28,127,128]$.

The early work of Plsek and Greenhalgh [28] defined a complex adaptive system as "a collection of individual agents to act in ways that are not always totally predictable, and whose actions are interconnected so that one agent's actions changes the context for other agent" ( $p$ 625).

The World Health Organization (WHO) [129] defined the health system as a system "that consists of all organisations, people and actions whose primary intent is to promote, restore or maintain health" and its goals are "improving health and health equity in ways that are responsive, financially fair, and make the best and most efficient use of available resources" ( $p 2$ ).

Using a complex adaptive system lens, the Institute of Medicine [27] defined a health system as "a set of connected or independent parts or agents, including caregivers and patients, bound by a common purpose and acting on their knowledge" (p 63).

According to Plsek and Greenhalgh [28], a mechanical system has fixed defined boundaries, for example all the parts of a car are known, and each part has a defined function that makes the car 
functional. A complex adaptive system is an open system with blurred boundaries, which has a large number of agents who can simultaneously be members of several sub-systems and the membership can change $[28,29]$.

The system agents or actors include health care professionals, managers, and patients and their families who learn and adapt from their experience and interactions in the system [27]. Carmichael and Hadžikadić [9] explain that these agents are independent, acting with local knowledge only but possess similar attributes (are self-similar) and therefore, are easily replaced by similar agents from within the larger system without disrupting the emergent features of that system. For example, all doctors are different individuals but because of a degree of selfsimilarity, they behave homogenously as an agent in the health system.

This homogenous behaviour and the correlated feedback among the agents create an internal set of rules or emerging patterns, which is sometimes referred to as 'flocking' [9]. This 'flocking' gives the system its emergent property and drives the actions and behaviours of agents $[9,28,29]$.

The actions and behaviours of agents create further interactions that influence future interactions. The system and the agents both learn and adapt as a result of these constant feedback loops, making the system unpredictable $[9,28,29]$. Future interactions cannot be predicted from past interactions and history cannot be undone, but history influences present interactions $[29,128]$.

The emergent property of a complex adaptive system also gives the system its self-organisation or generative property $[29,128]$. There is no central or external control on the flow of information in a complex adaptive system and system-level adaptation is not directed by hierarchy. Selforganisation is triggered by individual agents at the micro-level while following the internal set of rules and acting with their local knowledge [9]. Agents provide feedback to other agents and influence them to act in similar ways, which produces aggregated behaviour (like a snowball), and self-organisation emerges from this collective behaviour leading to system-level adaptation at a macro-level $[9,28,29,128]$. Carmichael and Hadžikadić [9] use the analogy of a flock of birds' response to seeing a predator: "the flock may shift and split apart as the individual birds try to avoid the predator, and these birds influence their neighbours to change direction as well. Even though no individual bird has changed how it reacts to seeing a predator - i.e., they haven't adapted or evolved - the flock itself can adapt to avoid the danger." ( $p$ 10) 
According to Carmichael and Hadžikadić [9], this system-level adaptation gives a complex adaptive system its power: "collectives reacting intelligently to the environment, with complex dynamics and versatility, even though they are comprised of simple agents with simple rules" ( $p$ 10).

Figure 4.1 illustrates a simple representation of complex adaptive systems, adapted from The Health Foundation [128].

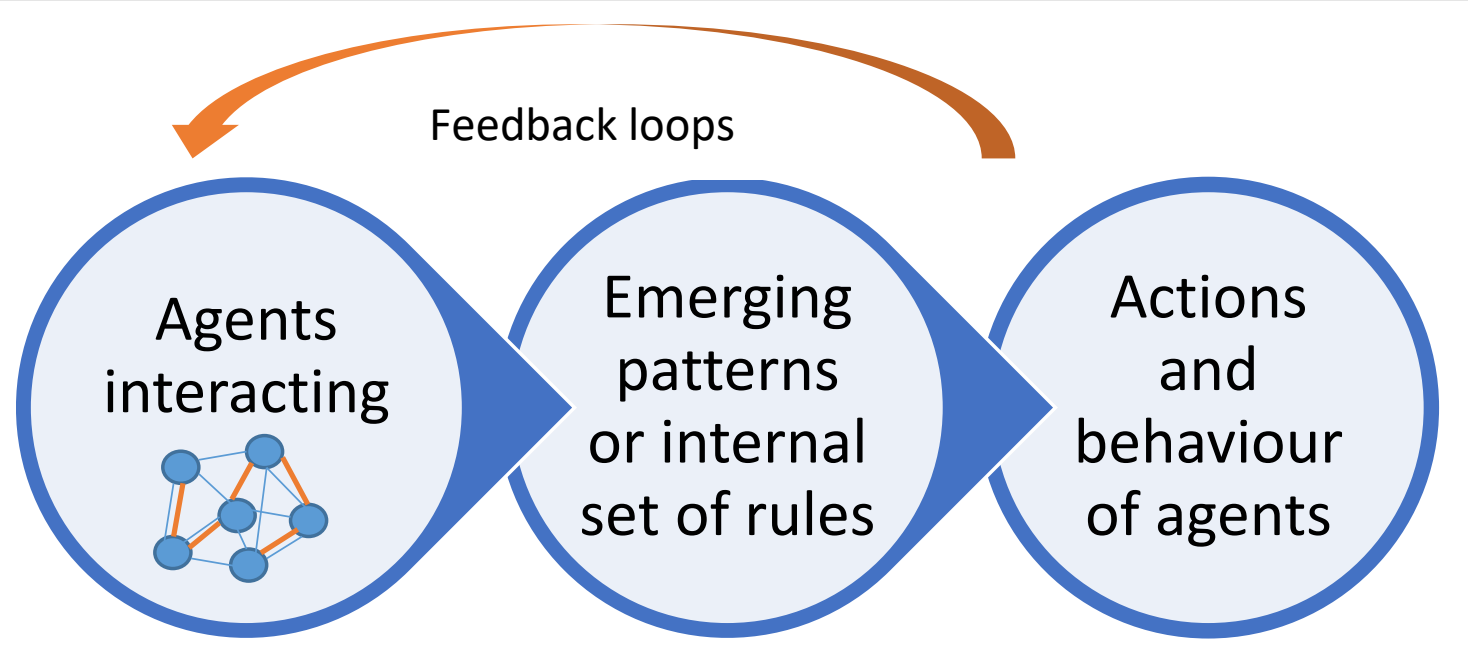

Figure 4.1: Simple representation of complex adaptive systems, adapted from The Health Foundation [128]

Sturmberg [5] used organisational theory to describe the complexity of the health system that comprises four levels: the macro level, which includes national policy and governance; the meso level that includes regional and local infrastructure and services; the micro level that is providing health care to an individual or local community; and the nano level that describes health and disease characteristics. Each level is further made up of multi-level components with multiple sub-systems nested in the wider health system. These systems interact and co-evolve and affect and are affected by each other. As the environment changes, the systems change to adapt to the environment, which in turn influences the wider environment and the constant cycle of change continues [128].

Scholars emphasise that the outcome of a complex adaptive system is a product of the interactions between the systems and the agents within those systems, making the system greater than the sum of its parts $[5,9,11,29,34,130,131]$. The properties of complex adaptive systems emerge from interactions and not from individual agents $[5,29]$. 
For health care organisations, this has implication for how goals are developed and how resources are pooled towards the system as a whole. So, instead of having separate budgets and performance targets for primary care and secondary care, the successful implementation of LST initiatives and achievement of system transformation relies on having a shared vision for the system that agents work together to achieve $[7,8]$. Implementation efforts are likely to fail for those seeking to direct change from the top using reductionist thinking that attempts to break down ambiguity to achieve more certainty, follow rigid protocols, separate and manage health care organisations or agents individually, and troubleshoot and fix clinical and organisational problems [28].

In summary, complex adaptive systems are complex open systems with blurred boundaries, whose agents interact, learn and adapt their behaviours to a changing environment. The constant interactions make the system emergent, unpredictable and generative [5-9]. Box 4.1 summarises three key features of a complex adaptive system from the works of Plsek and Greenhalgh [28], Holden [7], The Health Foundation [128] and Carmichael and Hadžikadić [9].

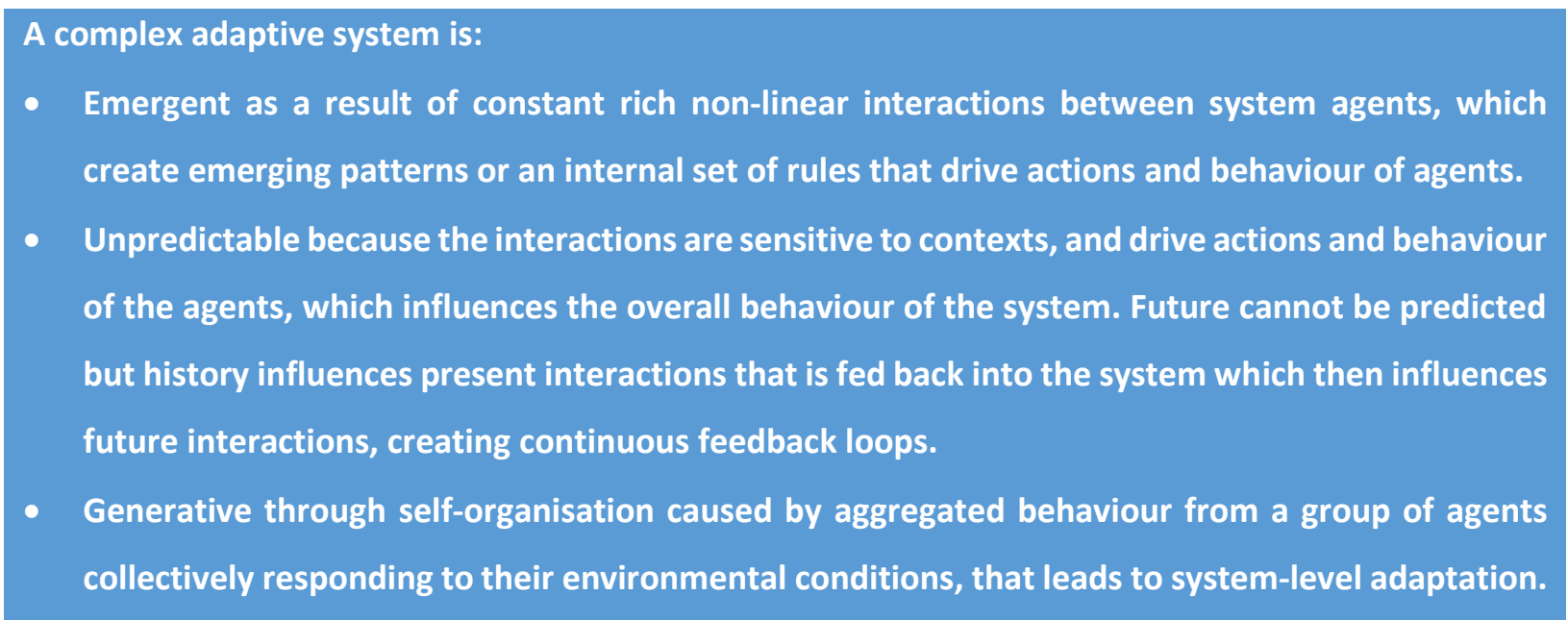

Box 4.1: Key features of complex adaptive systems

The macro framing of complex adaptive systems allows health system leaders and implementers to understand why implementation and the outcomes from LST initiatives vary between theory and the real world, and how implementation of these initiatives adapt to the local environment. It enables leaders and implementers to capture unanticipated results that naturally emerge from the dynamic interactions of the agents in the system, and these in turn may lead to the creation of innovative ideas to successfully implement LST initiatives and achieve the desired system transformation [9]. 


\subsection{Improving health system performance}

There are continued attempts to change the health system in order to improve the efficiency of the system, improve health outcomes and reduce health inequities. Traditionally, these attempts included organisational changes, and implementation of new national policies and performance frameworks. These attempts also include quality improvement initiatives such as reducing readmission rates to hospital by working with community health providers to follow up care with patients in their homes post-discharge or ensuring medicines use is effective and efficient via medicines reconciliation projects by local general practice teams working with their pharmacy partners. Perla, et al. [30] found that most quality improvement initiatives are usually small-scale and limited to a meso, micro or nano part of the system - for instance, they take place in a hospital or general practice setting, or focus on a health condition such as diabetes - and are difficult to scale-up and spread. Braithwaite [11] and Institute of Medicine [27] highlighted that despite numerous organisational and policy reforms, health systems world-wide are struggling with the rise in health inequities and health expenditure.

Scholars emphasise that traditional organisational and national policy changes assume a closed system, do not consider the emergent, unpredictable and generative nature of the system, and often use top-down approaches that are not always successful or sustainable $[6,11,27]$.

Braithwaite [11] offers caution with health system restructures, as these change organisational structures but do not change the entrenched cultures, behaviours or clinical outcomes. According to Braithwaite [11], change in the system is accepted when it is driven by evolving technology for diagnostic tests and treatments; scientific evidence that shows public benefit such as immunisation; emergence of new models of care such as virtual consults; and professionally driven change in clinical practices such as laparoscopic techniques. Change in the system is rejected when it is mandated from top-down; is not supported by powerful groups such as the medical profession; when it encounters entrenched bureaucracy; and does not attempt to address entrenched politics, behaviours and cultures.

Braithwaite [11] does not blame any person, group or organisation for what they describe as the inertia in the system but highlights that change in a complex system cannot be mandated through introduction of policy, regulation, restructure or performance indicators. Instead, they recommend building momentum for change by nudging the system towards one that is learning and where those working in the system understand the features of complex adaptive systems. These features include recognising the emergent, unpredictable and generative nature of the 
system and the system agents' ability to resist, ignore or adapt top-down mandates. Braithwaite [11] summed it by saying that "health care is governed far more by local organisational cultures and politics than by what the secretary of state for health or a remote policy maker or manager want" (p 2).

The landmark report from the Institute of Medicine [27], Crossing the Quality Chasm, called for a fundamental shift in the delivery and management of health care from one that is designed around health institutions and health care professionals to respond to acute episodic care to a system that is people-centred, delivers seamless co-ordinated holistic care, and one that acknowledges and addresses health inequities. The report [27] outlined the design of the health system for the $21^{\text {st }}$ century to deliver health care that is safe, effective, patient-centred, timely, efficient and equitable and a system that supports innovation and continuous improvement. The report endorsed the notion of health systems being complex and acknowledged that system transformation with collective leadership will be required for the desired shift.

Pratt, et al. [75] highlighted that those in the system have become used to the language of mechanical systems such as cogs and wheels, targets, levers and that attention is instead required to features such as connections, relationships and meaning to recognise health as a living eco-system.

Martin and Félix-Bortolotti [132] concluded that "making a paradigm shift from many centuries of reductive approaches to reduce certainty in complex non-linear systems is a challenging exercise yet becomes increasingly imperative" ( $p$ 419).

Holden [7] emphasised that "the application of the understanding of health care as a complex adaptive system involves cultivating an environment of listening to people, enhancing relationships, and allowing creative ideas to emerge by creating small non-threatening changes that attract people" (p 656).

According to Baker and Axler [122], high performing health care systems "have created effective frameworks and systems for improving care that are applicable in different settings and sustained over time" ( $\mathrm{p} 4)$. This creation requires a deliberate and systemic approach and a shift away from reductionist thinking to understanding that attempts to change the performance of the health system need LST initiatives that engage multiple organisations and a focus on relationships, interactions and the behaviours of those working in the system. 
Reviewers of health systems over last the two decades have repeatedly emphasised that LST initiatives based on relationship and trust rather than transactions and directives, with clinical governance and continuous improvement focus is more likely to be enduring and sustainable $[1,5,6,11,32,37,99,130,133-135]$.

\subsection{Informal networks}

One of the key features of complex adaptive systems is the emergence of informal networks from the interactions and connections between agents in the system [5,135,136]. According to Khan, et al. [33] and The Health Foundation [137], these informal networks facilitate adaptation and have the potential to transform the system by creating a platform for learning, leading and spreading LST initiatives.

In research commissioned by the Health Foundation [137], analysis of seven types of networks in health care found five core features of effective networks: common purpose, cooperative structures, critical mass, collective intelligence, and community building. When these five features interact, their combined effect generates the energy and momentum for change. The Health Foundation [137] defines a network as "a cooperative structure where interconnected groups or individuals coalesce around a shared purpose on the basis of trust and reciprocity" ( $p$ 7) that can bridge gaps between professional groups and competing organisations and be used to implement LST initiatives. Clinicians and leaders can have significant influence on the attitudes and behaviours among their colleagues and networks $[11,138]$.

Scholars of health system highlight that creating an environment that harnesses relationships, and the capability and capacity for improvement is more likely to provide ideal conditions for LST initiatives $[1,32,138]$. This environment enables use of improvement science and tools such as plan-do-study-act (PDSA) cycles to shift the paradigm from meeting targets to one that supports a learning system, engages frontline staff from across the system, removes hierarchical structures, uses local knowledge and provides a neutral setting for continuous quality improvement [1,137-139]. Clinicians are more likely to engage when they are empowered, nurtured and influenced by their peers in an environment that promotes continuous quality improvement rather than directed or controlled through hierarchical structures $[11,32,138]$.

According to Serra, et al. [69], delivery of patient-centred services requires a deep commitment and collective responsibility among all system agents along with agreed clinical pathways and a common purpose. The informal, trust-based networks nurtured by aligned interests that serve 
the interests of the community while preserving the autonomy of individual organisations can provide an effective and transparent governance model for the health system. For such networks to be successful, more attention is needed to be paid to developing strong and sustainable relationships, trust and goodwill than to structures and processes. Often these networks fail because culture is seen as a soft or 'touchy feely' matter; and there is an underestimation of time and effort needed to sustain the network, a limited shared understanding among the network partners, and unrealistic timeframes and expectations of what the network can achieve or deliver [69].

Use of informal trust-based networks to deliver integrated patient-centred care is emerging in health systems worldwide. These networks are often referred to by different names; such as integrated care systems or networks [72], accountable care organisations [73], and place-based or place-led partnerships [74]. Regardless of the name, the underlying philosophy of these networks remain the same: unprecedented levels of trust, strong and sustainable relationships, a common purpose, a shared commitment, and guided by principles and practices of adaptive leadership.

\subsection{Large-system transformation initiatives}

LST initiatives are initiatives designed to achieve system transformation. These initiatives use a complex adaptive system lens and embrace a systems mind-set. Reviewers highlight that a systems mind-set brings the agents in the system together and shifts the thinking from a linearcause-and-effect paradigm to a multi-causation-effect paradigm, and take into consideration the contexts in which these initiatives are implemented [31-34,78,132,140,141].

Best, et al. [1] found that implementation of LST initiatives requires organisations to employ change management theories and models to make changes meaningful and sustainable. Stouten, et al. [142] point out that there are many theories and models of change management and a lack of consensus on basic change management processes and principles. This can lead to organisations feeling overwhelmed by the options available and the steps they need to take. Many of the change theories and models are from a time when change was predictable and at a slower rate with a lack of technology, shorter life expectancies, less focus on health inequities and therefore less demand for transformational change. The increase in demand for health services with greater longevity and complexity in health needs, significant advances in the use of technologies, the changing expectations from the public to be treated as a consumer of health services, less tolerance of health inequities and increasing financial pressures mean that changes 
in the health system are now faster and more disruptive. Use of traditional organisational hierarchy to implement transformational change is too slow and risk-averse [32]. Braithwaite [11] advocates for health systems to use a complex adaptive system lens to "move beyond today's frozen systems performance" ( $p 3$ ) to new thinking paradigms; and from a linear causeand-effect approach to a continually learning system. This shift requires the leaders of the system to use informal networks to build high-trust relationships between agents in the system, identify shared vision and goals, and use feedback loops for constant learning [11,32,141].

A practical guide for leading LST initiatives developed by NHS England [32] endorse that transformation change in a complex adaptive system must take into consideration the emergent, unpredictable and generative nature of the system; mobilise a large number of individuals and organisations to work towards a common shared purpose; and require a shift in power through a distributed leadership. According to the practical guide, successful LST initiatives lead to "such deep changes in attitudes, beliefs, and behaviours that sustainability becomes largely inherent" (p 12) [32].

Best, et al. [1] offer a definition for LST initiatives in the health system: "interventions aimed at co-ordinated, system wide change affecting multiple organisations and care providers, with the goal of significant improvements in the efficiency of health care delivery, the quality of patient care, and population-level patient outcomes" (p 422). It is, however, difficult to find examples of LST initiatives in the health system that fit this definition; the literature is saturated with changes that focus on health conditions and/or in a part of the system such as hospital or general practices. Bevan and Fairman [35] argue that these small-scale and service improvement initiatives are important, however are not enough to achieve the desired shift in the health system. The three key aspects of LST initiatives are that these are broad and widespread across geographical boundaries, multiple organisations or across professional groupings (pervasiveness); these challenge current way of thinking and seek paradigm shifts in mindsets, processes and relationships (depth); and these affect people and require co-ordination across multiple systems nested within a macro system (size) [32].

In a complex adaptive system, the successful implementation of LST initiatives are challenging as they are dependent on behaviours, interactions, and social and cultural conditions of the system and changes are difficult to spread broadly and rapidly [30]. According to scholars of health system, success is dependent on having a clear mandate for change, a presence of right blend of leadership, dedicated resources, active engagement among staff, repeated attempts at change 
with feedback loops that enable learning, unlearning and adapting from experience, and patience from the leaders of the system for long-term gains [1,32,130,141-144].

From the key principles identified by Sturmberg [5], a set of simple or operational rules that act as system enablers for implementation of LST initiatives is necessary to foster collaboration across the system. These rules are variously referred to in the literature as key factors, conditions, themes, attributes or elements. Appendix 2 summarises published and grey literature on LST initiatives, five of which are discussed in detail in Chapter 9 (section 9.2); these are used to examine the key elements that increase the chances of success with implementation of LST initiatives.

Khan, et al. [33] and the practical guide developed by NHS England [32] emphasise the importance to understand the contexts in which transformation is implemented, whether it is top-down and restricted by organisational and professional boundaries ('old power'), or a collaborative approach using informal networks ('new power'). These two approaches create different conditions for successful implementation of LST initiatives.

\section{5 'Old Power' and 'New Power'}

Strategy\& (formerly known as Booz \& Company), a consulting unit of PricewaterhouseCoopers, conducted one of the largest study from a range of industries in Americas, Europe, Asia-Pacific, the Middle East and Africa to survey the role of culture in organisational change [145]. Industries included in the study were retail, health care, finance, energy and engineered product services. The study, involving over 2,000 executives, managers and employees, reported that about half of LST initiatives fail or are not sustainable owing to three major reasons: change fatigue as the workforce runs out of energy, lack of capacity and capability, and a 'top-down' approach that does not engage the frontline workforce.

Heimans and Timms [13] introduced the idea of 'old power' and 'new power' for the corporate world where traditional leadership is being challenged with an emerging trend of harnessing energy through co-producing, peer co-ordination, informal networks, collaboration, building capacity and capability, sharing and transparency for leadership and transformational change. Figure 4.2 illustrates the difference between 'old and new power' that has been reproduced from Heimans and Timms [13], and Bevan and Fairman [35]. Bevan and Fairman [35] use 'dominant approach' and 'emerging direction' to describe the 'old' and 'new' powers respectively for the health care setting. 
In the 'old power' approach, change is mandated from the senior operational leaders who exercise the power of their positions and seek to achieve the mission and vision of the organisation. In a 'new power' approach, power comes from relationships, connections and the ability to influence through informal networks and communities to achieve a shared purpose [32]. 'New power' has an emotional connection that uses people's intrinsic motivation to call for action with an emphasis on social resources that grow with use whereas 'old power' relies on economic resources that diminish with use such as funding and materials [13,32]. Most importantly, the 'new power' approach engages patients, families, communities and frontline health care professionals in transformational change.

\section{Old Power}

\section{New Power}

- Financial management

- Accountability

- Targets

- Sanctions

- Leader-driven

- About economic resources (\$\$, materials, technology which diminish over time)

- Important part of the system but not the whole

- Closed system

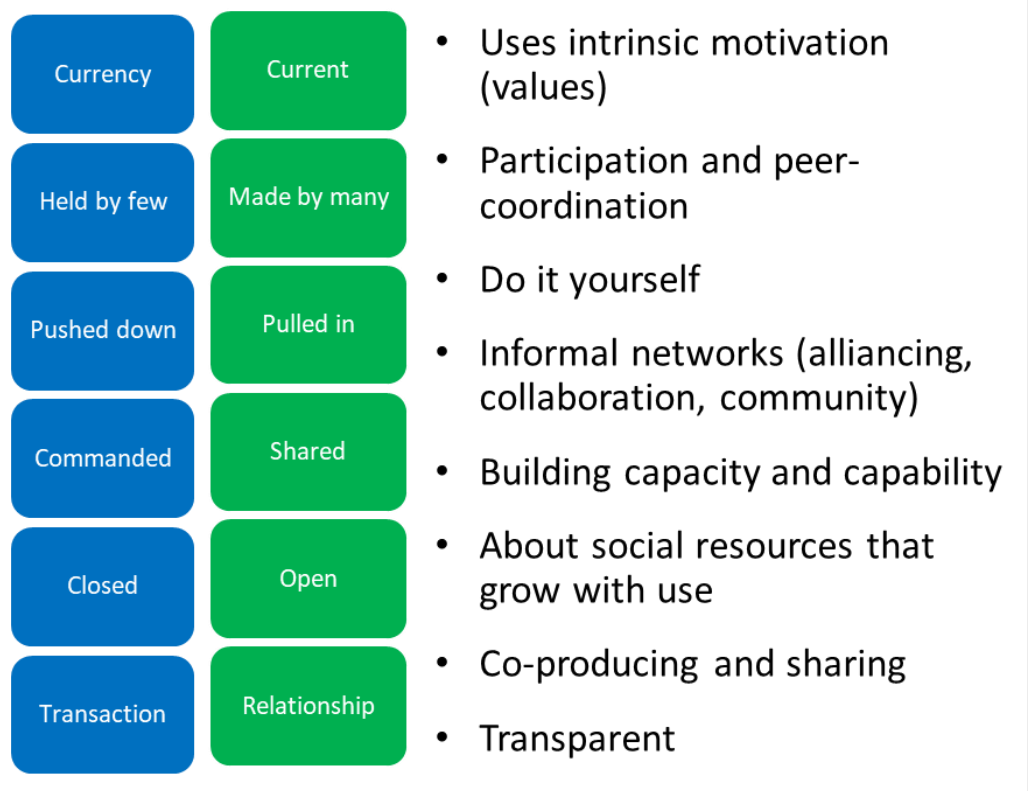

Figure 4.2: Illustration of 'Old and New Power', adapted from Heimans and Timms [128] and Bevan and Fairman [126]

Historically and currently, health care organisations and government agencies such as the $\mathrm{MoH}$ predominantly use the 'old power' approach to develop and implement strategies, policies and change interventions through accountability frameworks, performance measures with national targets, incentive payments or pay-for-performance schemes, compliance and quality assurance. This traditional fragmented approach to managing health system performance reinforces the tendency of health care organisations to be preoccupied with power, rules, positions, and organisational and departmental patch protection. Critics of traditional approach argue that to successfully implement LST initiatives in a complex adaptive system, this approach is no longer fit for purpose [32,35]. 
The 'new power' approach requires all agents in the system to focus on the mission or vision of the system, use collaboration and partnership approaches and be generative with the use of continuous feedback loops [146]. It harnesses energy from and among clinicians, managers, patients and communities through collaboration, shared common purpose and high-trust relationships to achieve the desired system transformation [11,32,138].

In a publicly funded and institutionalised health care system, it is challenging to shift the paradigm from 'old power' to 'new power' as elements of 'old power' are ingrained as a way to demonstrate accountability of the system to the public and the government.

\subsection{System accountability}

Political salience or visibility is high for health systems because many are predominantly funded from general taxation and there is an expectation from the public for the system to demonstrate value for money. Additionally, there is high interest in achieving good health outcomes of people and communities from public and health care professionals. For New Zealand, in a Westminsterderived system, citizens and opposition MPs hold Ministers accountable for delivery of public health services, and this includes 'fixing' problems or answering for the Ministers' 'performance failure' [50,51]. Cook and Huges [51] claim that expectations from the public and opposition MPs have led to a high degree of Ministerial direction on operational matters and accountability of public services has evolved to accountability to Ministers rather than citizens, making Ministers the clients of the public sector organisations. The high political salience makes it difficult for governments and the health system to solely embrace the 'new power' approach because of ingrained 'new public management' ideas to demonstrate efficiency and accountability of the system.

For instance, following a change of government in 2017, the new Minister of Health ceased public reporting of six national health targets [147], which generated a number of mixed responses from professional groups, opposition MPs and the public. The media headlines that followed this announcement included: "Health experts divided over changes to national targets" [148] and "National warns of more deaths with no public health targets" [149].

System accountability is an important feature of public institutions and is used to assure external stakeholders, including the public, of the legitimacy of the organisation and that the system is meeting the required performance standards. Gill [50] explains that performance management consists of rational control that measures performance of an organisation against a standard, 
monitors deviations from the standard and takes action to reduce deviation from the standard. Use of rational control punishes deviations, ignores political and cultural context in which the system is operating, and system agents retreat from learning to focus on following the rules. Gill [50] goes on to say that there is no opportunity to innovate and learn with a rationalist model and the emphasis is on 'was it done right'. System accountability is aligned with the 'old power' approach.

System performance, on the other hand, focuses on continuous learning taking into consideration the political and cultural context. According to Gill [50], organisations depart from the rules to achieve the outputs and outcomes, use variation to innovate and the emphasis is on 'was the right thing done'. System performance is aligned with the 'new power' approach.

Reviewers argue that the dichotomy of system accountability and system performance creates tension in the system $[20,50,51]$. System accountability is required to meet the legislative and the Cabinet requirements. In complex adaptive systems, a continuous learning approach that focuses on interactions, behaviours and social and cultural contexts is needed to improve the system's performance and health outcomes $[11,29]$.

Heimans and Timms [13] proposed a 'bilingual' approach that develops capacity and capability for both 'old' and 'new' powers. They introduced the 'new power' compass that distinguishes between the 'old and new power' operating model and values. For example, technology companies such as Facebook, operate under the 'new power' business model as their success depends on participation. However, they sometimes make decisions that ignore the wishes of their users; this is aligned with 'old power' values. Conversely, companies can operate with a traditional 'old power' business model but embrace 'new power' values like transparency [13]. The 'new power' compass helps organisations identify operating models and values they want to change and adopt strategies to deploy 'new power'. Heimans and Timms [13] urge that 'old power' organisations such as health systems that are ingrained in 'new public management' must think differently and embrace 'new power' values to improve the performance of the system.

A white paper published by a national improvement body for NHS [35] stated that current and future challenges facing health systems cannot be addressed with sole use of an 'old power' approach and leaders need to recognise the emergence of 'new power' values to successfully implement LST initiatives. 
Scholars advocate that instead of just focusing on process, measurement and execution, health system leaders need to use complexity thinking and embrace connections, engagement, shared purpose and relationships, and be bilingual with both powers as both are essential and both are needed to improve performance of the health system $[13,35,150,151]$

My insights based on observations during implementation of the SLM programme revealed that health system leaders are overwhelmed with change management theories and are unclear on steps they need to take to successfully implement LST initiatives. I recognised that a selfassessment maturity matrix is one way to help identify the programme architecture that underpins efforts to successfully implement LST initiatives. The self-assessment approach could support the notion of a learning system and increase understanding of this programme architecture for health system leaders and networks such as Alliances.

\subsection{Use of maturity matrices}

A maturity matrix is a useful learning tool to show how individuals, organisations and networks can 'get there from here'. It can be used to provide feedback on performance of a person or an organisation through audit, and self-, peer- and external assessment. Audit and external assessments are used to demonstrate assurance, accreditation or compliance and often have incentives linked to achievements, for example, financial rewards or quality accreditation and renewal of professional licences or certifications [151,152]. The self-assessment approach is used to stimulate individuals, organisations or networks to identify areas of improvement and develop an action plan that includes goals for improvement, and identifying the barriers and enablers to achieve the goals $[151,152]$.

There is ample evidence on the use of maturity matrices in health care setting, however most of these include professional competency frameworks and organisational accreditations such as general practice accreditation standards $[153,154]$. Researchers argue that these frameworks are perceived as compliance exercises, do not use a plan-do-study-act cycle for improvement and create tension between quality control, regulation and quality improvement $[150,154]$. According to Rhydderch [150], improvement in health systems needs to be continuous, valuesdriven and bottom-up; which requires a shift from traditional accreditation approaches. Research from the United Kingdom and Europe shows the emerging use of maturity matrices in health care settings to adopt complex innovations (such as LST initiatives) and sustain these long-term at organisation or system level [155-161]. 
The concept of a maturity matrix with a self-assessment approach to stimulate quality improvement in the health care setting was initiated in 1996 as part of a doctoral thesis and was adapted for use in Denmark [153,154]. Buch, et al. [152] evaluated the use of the maturity matrix in Danish general practices developed by Elwyn, et al. [153]. The authors found that the maturity matrix was a good starting point for improved communication and co-operation within the practice team. However, organisational factors such as the size of the general practice, high patient-related workload and staff turnover influenced the implementation of improvements identified following the self-assessment. Other factors that influenced implementation were: the availability of an external facilitator who understood the matrix and helped the general practice with the self-assessment; allocation of anchor persons who kept the implementation process active; a lack of perceived benefit; and a lack of formal commitment [152].

Edwards, et al. [151] undertook a feasibility study of developing a maturity matrix for quality improvement in the European primary care setting that included 153 practices based in the UK, Germany, The Netherlands, Switzerland and Slovenia. The authors concluded that the maturity matrix was a feasible and valuable tool for practices to assess their organisation development and planned improvements to improve health care quality. Elwyn, et al. [154] later refined this maturity matrix using an expert panel of primary care practitioners followed by testing in five primary care practices. This study was the first to develop an international maturity matrix for quality improvement in primary care.

In 2011, the Development Model for Integrated Care (DMIC), a web-based self-evaluation, tool was developed by a doctoral student Mirella Minkman [162]. The DMIC tool was used by integrated care practices in the Netherlands to evaluate their delivery of integrated care, to assess their readiness for delivery and to identify areas of improvement. The tool contained 89 elements grouped in nine clusters: client-centredness; delivery system; performance management; quality of care; result-focused learning; interprofessional teamwork; roles and tasks; commitment; and transparent entrepreneurship. The tool was validated by testing in 84 integrated care services in the Netherlands to assess the relevance and implementation of the elements.

The Scaling Integrated Care in Context (SCIROCCO) is a project co-funded by the Health Programme of the European Union $[163,164]$. One of the outcomes of the project was the SCIROCCO Maturity Model, which is an online self-assessment tool that enables those working in the health and social care system across the European Union to assess their readiness to deliver 
integrated care. The tool aims to help European regions to understand their strengths, weaknesses and potential areas of improvement; adopt and scale up integrated care solutions; facilitate multi-stakeholder discussions on progress and delivery of integrated care; and facilitate coaching to help regions and organisations understand the local conditions that enable successful delivery of integrated care [163]. One of the important components of this model is that regions share their experience and assessments with others through a web-based platform. This facilitates sharing of knowledge between the regions. The self-assessment tool is made up of 12 domains (Box 4.2) with an assessment scale for each domain. Initial testing showed the tool to be effective in revealing the maturity of the health care system context for providing integrated care [164]. However, the tool is still in early stages of implementation and further studies are recommended to confirm its structural validity.
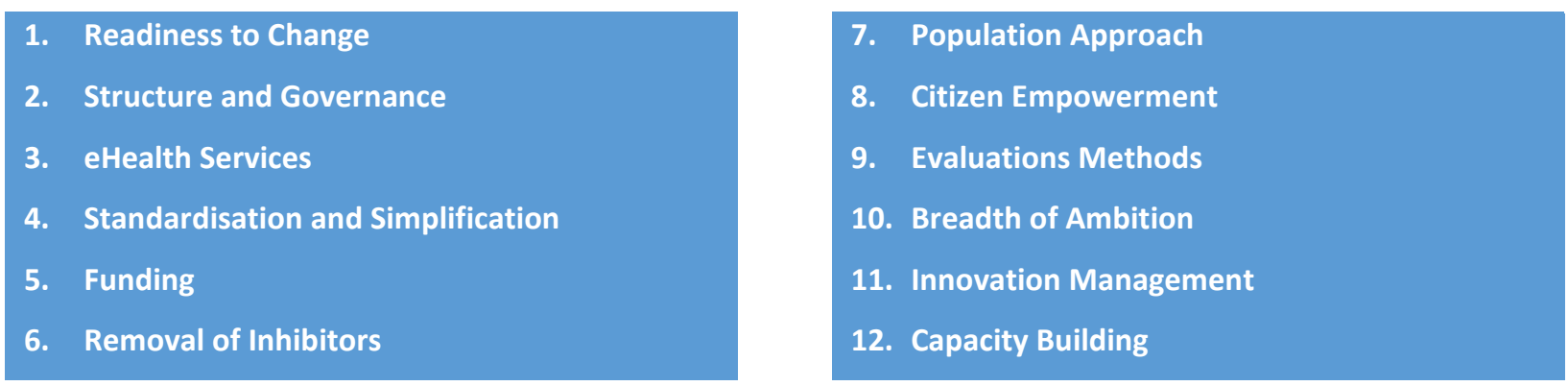

Box 4.2: SCIROCCO maturity model dimensions

The evaluations on the use of maturity matrices confirm that a self-assessment maturity matrix can be a useful tool to support implementation of LST initiatives as it helps health system leaders recognise their strengths and weaknesses and identify areas of improvement needed for system transformation [151,152,154,161,162,164]. 


\subsection{RESEARCH ISSUES, AIMS AND QUESTIONS}

Academic literature leads me to the conclusion that health care systems are living and adaptive complex systems in which relationships, connections and interactions influence the behaviour of those who work in and use the system. The constant interactions make health systems emergent, unpredictable and generative $[7,9,28]$. Organisational and financial accountability of health service providers is integral to demonstrate public and government confidence for a system that is predominantly taxpayer funded. However, organisational reforms, 'new public management' practices and small-scale change efforts at the micro level are not enough to improve the performance of the system. LST initiatives that capitalise on the key features of complex adaptive systems such as the use of informal networks and 'new power' values, together with a deep understanding of the contexts and mechanisms that influence LST initiatives, may be more likely to achieve the desired system transformation $[1,11,32,35,37]$. However, health system leaders feel overwhelmed with change management theories and organisational changes and are unclear on the steps they need to take to successfully implement LST initiatives.

The SLM programme is an LST initiative designed to enhance a collaborative way of working beyond organisational and professional boundaries, address health inequities, and encourage continuous learning and quality improvement. My insights and experience from leading the implementation of the SLM programme and the evaluation of the programme [121] revealed some of the architecture that underpins efforts for successful implementation of LST initiatives. This programme architecture includes a set of key elements that need to be present in the health system to increase the chances of success with implementation of LST initiatives. These elements are sensitive to contexts and influence implementation of LST initiatives. Additionally, the insights and the evaluation revealed that this architecture for LST initiatives is not well understood among the system leaders and the Alliances in the New Zealand health system.

The successful implementation of LST initiatives involves creating positive conditions for change to support a work environment that harnesses relationships and increases the capacity and capability of those working in the system. It is less about meeting LST initiative targets and is more about iterative planning and practice cycles to shift system behaviour. Evidence of successful implementation includes rich descriptions about 'what works, for whom, under what circumstances, and why'. 
Academic literature and my experience with implementation of the SLM programme raised the following questions:

- What is the role of LST initiatives such as the SLM programme in supporting the New Zealand health system to bring all agents in the system together to work towards a common purpose?

- What are the key elements that support successful implementation of LST initiatives and form the architecture of these initiatives?

- What do these key elements look like for New Zealand health system?

- Why does implementation of LST initiatives vary at district level, i.e., how do the key elements work in different contexts?

- How can system leaders and Alliances assess their capacity and capability for successfully implementing LST initiatives?

- How can the health system be consciously and deliberately supported to become a learning system for successfully implementing LST initiatives?

These questions motivated me to design a research project to more explicitly reveal the programme architecture that underpins efforts to successfully implement LST initiatives in the New Zealand health system. From my insights leading the implementation of the SLM programme, I theorised some of the key elements that need to be present in the system to increase the chances of success with implementation of these initiatives: an alliancing way of working; clinical leadership and engagement; the degree to which consumers were involved in the development and implementation of the SLM plan; information technology infrastructure; the capacity and capability for quality improvement; and the use of commissioning cycles. From my insights, I also theorised some of the contextual factors that influenced the implementation of LST initiatives: maturity of the Alliances; quality of local relationships; the presence of a shared vision for the district and a commitment from system leaders to work towards that vision; and the leadership styles of the $\mathrm{MoH}$ and DHBs.

I identified a self-assessment maturity matrix as one way to describe the elements along a scale so Alliances can see what good looks like for these elements, assess where they are on the scale and identify areas for improvement. Research literature suggests that the self-assessment process will support the Alliances to consciously and deliberately increase their understanding of the programme architecture for successful implementation of LST initiatives. The design also included investigating and reporting on how the key elements work in different contexts and 
influence implementation of these initiatives. Figure 5.1 shows the nested approach to the research.

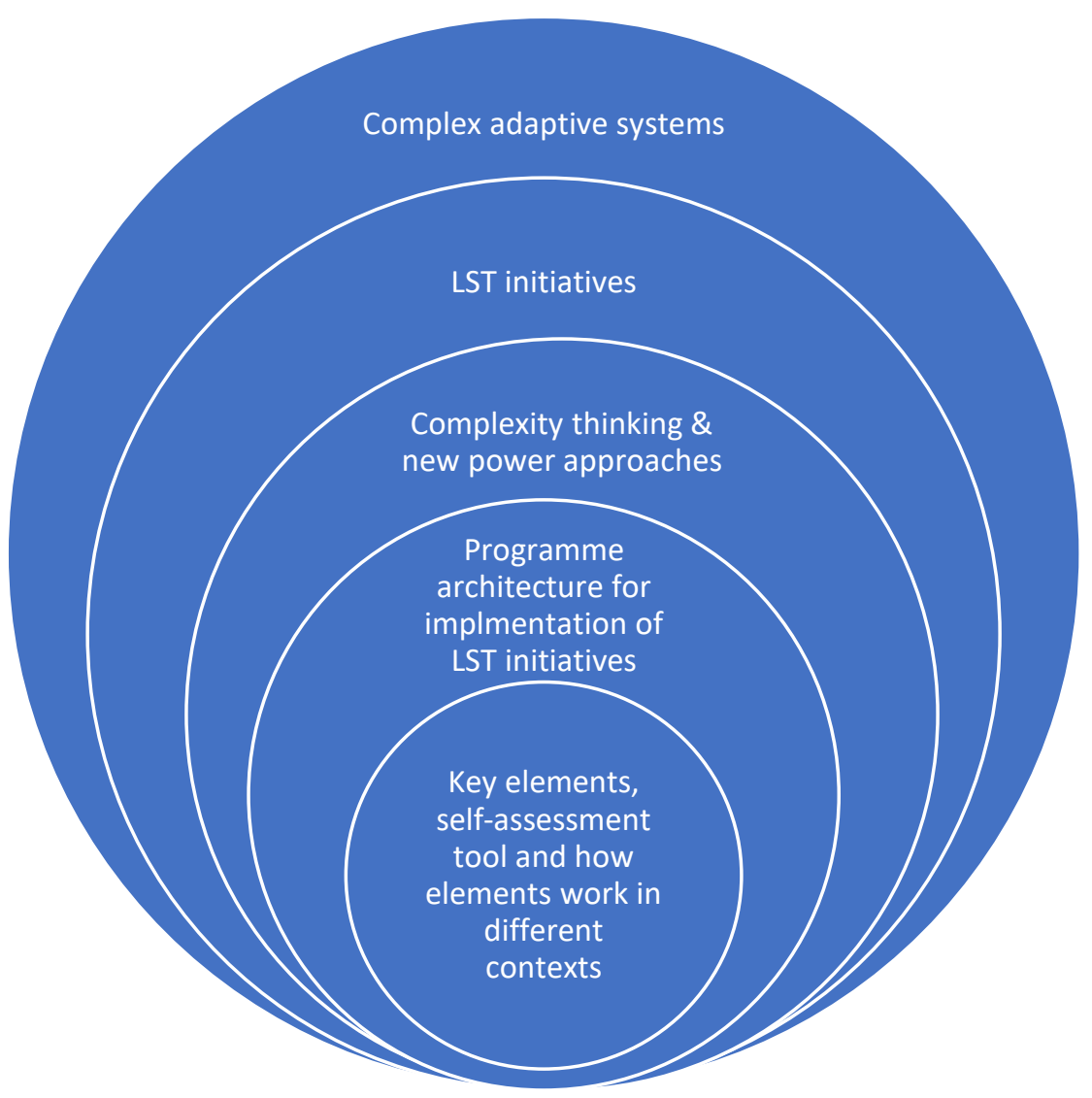

Figure 5.1: The nested approach to the research

\subsection{Gaps in knowledge}

A reasonable amount of literature on applying complexity thinking to health systems has emerged in the last two decades. However, there are very few studies where complexity is applied with LST initiatives that require all agents in the health system to work together [6]. Furthermore, there are even fewer studies of how contexts and mechanisms influence success of LST initiatives [1], although the number of studies using a realist approach to demonstrate the role of contexts and mechanisms is increasing $[44-46,165,166]$. However, majority of these studies are at micro level and do not extend to a systems mind-set or explore inter-relationships between agents of the system. A search of OVID and PUBMED databases for all realist evaluations, research, reviews and synthesis, over last five years (2015 to current) revealed 767 published articles. When the search was filtered with 'system or large-system transformation', only 13 studies were identified. Extending the search to 2000 revealed additional five studies. On closer examination of these 18 studies, 10 discussed system transformation, each with different a perspective of what a large-system transformation is, and none of the studies were from New 
Zealand. Of 10, only two met the definition of LST initiatives used in this research. The rest were either at a meso or micro level or were realist rapid review of literature.

Similarly, studies on the use of maturity matrices for quality improvement or learning are also at meso or micro level of the health system [151,153-157]. Nothing exists in New Zealand that describes the architecture that underpins efforts to successfully implement LST initiatives in a way to support conscious and deliberate learning among the agents in the health system and increase the chances of success with implementation of these initiatives.

\subsection{Research aims}

This research had three aims: first, to identify the key elements that support successful implementation of LST initiatives; second, to construct a maturity matrix that describes different stages of maturity for each of these elements; and third, to investigate and report on how contextual factors that New Zealand alliances work in influence successful implementation of LST initiatives. Collectively, the three aims described the programme architecture that underpins efforts to successfully implement LST initiatives in the New Zealand health system.

\subsection{Research questions}

To achieve the research aims, the following three questions guided the research.

- What are the key elements that support successful implementation of LST initiatives in the New Zealand health system?

- How do New Zealand Alliances consciously and deliberately increase their understanding of the key elements to support the notion of a learning system?

- What contexts and mechanisms influence New Zealand Alliances' ability to successfully implement LST initiatives? 


\subsection{Summary of Part A}

Part A set the scene for the research. It started by describing the Westminster-derived New Zealand health system and how past reforms and 'new public management' practices have shaped the current health system. Like other health systems world-wide, the New Zealand health system is centred around health institutions and health care professionals to respond to acute episodic care. The system is now struggling to meet the changing demands with longer lifespans, a growing burden of long-term chronic conditions with an ageing population and persistent health inequities, in particular for Māori (the indigenous population of New Zealand). The New Zealand health system is seeking a fundamental shift in the way the health services are funded, managed, and delivered. Chapter 2 included key recommendations from a major review of the system that was commissioned by the government in 2017 in the endless quest to improve performance of the system.

Chapter 3 described the development and implementation of the New Zealand System Level Measures (SLM) programme. It included insights and knowledge gained from leading the implementation of the programme; these form the foundation of this research. Chapter 4 provided academic context of the research with a review of international literature. It described health systems as complex adaptive systems that are emergent, unpredictable and generative and that need LST initiatives to better achieve the desired system transformation. These ideas support the design and implementation philosophies of the SLM programme. The chapter identified the use of maturity matrix as a self-assessment tool to support the notion of a learning system.

Research in health system transformation is emerging internationally and the New Zealand health system is at a pivotal time when improvement in the performance of the system will require more than 'new public management' ideas and organisational changes. A more considered and sophisticated approach to system performance is needed with an understanding of the key elements that support successful implementation of LST initiatives in complex adaptive systems; and how these elements work in different contexts to influence the successful implementation of these initiatives.

Part A ended with the research aims and the three research questions that guided the research. The next part discusses the methodological frameworks, rationale for the research methodology chosen and the research design. 
PART B:
METHODOLOGY 
Part A established that LST initiatives that capitalise on key features of complex adaptive systems together with a deep understanding of the contexts and mechanisms that influence these initiatives, are more likely to achieve the desired system transformation.

My research questions led me towards needing particular types of inquiry frameworks that had strengths in uncovering system dynamics, provided a sound framework to test and refine initial propositions that emerged from the SLM programme with a wide range of stakeholders, and attended to the contextual factors that influenced successful implementation of the SLM programme.

Part B of the thesis contains three chapters.

\section{Chapter 6: Theoretical underpinnings}

This chapter explains the theoretical underpinnings and the rationale for choosing a realist logic of enquiry nested within the framing of complex adaptive systems.

\section{Chapter 7: Research design}

This chapter describes the research design that includes five qualitative methods and one quantitative method of data collection: theory gleaning from the SLM programme; a review of published and grey literature; interviews; workshops; an online survey; and field testing of the maturity matrix.

\section{Chapter 8: Ethical considerations}

The final chapter in this part of the thesis discusses the ethical considerations for the research that includes managing conflict of interest arising with my dual role as the SLM programme manager and researcher, research confidentiality and informed consent. 


\section{0}

THEORETICAL UNDERPINNINGS

Traditionally, positivist and constructivist paradigms have been applied to researching change in the health sector.

In a positivist paradigm, researchers perceive particular interventions will achieve an outcome and then use methods such as randomised control trials (RCTs) or before-and-after analyses to test or pilot interventions, which then forms the basis for most clinical practices and interventions $[6,48,167]$. Westhorp [168] explains that traditional research designs in this paradigm take a linear cause-and-effect perspective: "if we see $A$ and then we see $B$, we always see $B$ after $A$, and without A we do not see $B$, then A causes B" ( $p$ 407). Researchers control the contextual factors and take a prescriptive implementation approach. In doing so, they do not take into consideration the emergent, unpredictable and generative nature of a complex adaptive system and therefore make the study and its findings challenging to scale up or spread beyond the pilot site [6].

A positivist paradigm does not take into consideration the powerful influence of the system structure and the constant interactions between system agents that result in ongoing adaptation of the system $[6,167]$. This makes it difficult for evaluators of change to explain what caused the expected or unexpected outcomes and under which contexts, thereby making it a challenge to replicate the outcomes and make change sustainable $[47,166]$. Historical change efforts using this paradigm have focused only on parts of the health system, either at meso or micro levels, making some parts of the system perhaps more efficient but not the whole.

In a constructivist paradigm, findings rely on learning through social interactions and therefore reality is socially constructed during the research [169]. The close and constant interaction between the researcher and the participants seeks multiple views that may exist. These constructed realities create rich descriptive findings, however, may not always be useful for comparisons or generalisations. A constructivist paradigm is suitable and useful in some health research in which socially constructed reality is important to understand individual behaviours or outcomes, or where an open-ended inquiry is needed to formulate theory or theories. It is, however, less suitable for research that starts with initial theories and aims to test and refine these theories rather than build theories from the ground up [167]. The philosophical assumptions of the constructivist paradigm are that knowledge is constructed by researchers and participants during research through an interactive process [169]. The methodological 
implication is that research questions cannot be definitely determined before the study begins but rather evolve and change during research [169].

\subsection{Realism}

Realism, as developed by Pawson and Tilley [4], is a philosophy that sits between positivist and constructivist paradigms. It focused first on evaluation of discrete programmes and then moved to realist synthesis of secondary material and to realist research. The philosophical assumptions of this research are that health systems are complex adaptive systems in which relationships, connections and interactions between system agents create feedback loops. These feedback loops, along with contextual factors of the system, influence the successful implementation of LST initiatives. Realism theory offers a framework to uncover the capacity and reasoning of health system agents acting in social and cultural conditions of the system in which LST initiatives are implemented.

The realist approach is a way of thinking and a logic of enquiry that drives research design in which outcomes follow from mechanisms (capacity and reasoning of people) acting in the contexts (social and cultural conditions) of the system [4]. It can use qualitative or quantitative or both methods to test and refine programme theories to understand how mechanisms that operate according to context influence programme outcomes [4,170-172].

The realist approach draws on system and complexity theories, and reports on: 'what are the mechanisms that explain how and why reality unfolds as it does in a particular context' or 'what works for whom under what circumstances and why' [4]. In this research it offers a way to build on the lessons of the SLM programme and to test theories with a wide range of participants from across the health system (senior leaders to frontline staff).

\subsection{Systems theory}

Systems theory postulates that a system is both greater than and different to the sum of its parts; and that a system cannot be understood by studying its component parts as doing so loses the essential properties of the system. A system has many interdependent parts and it is these interactions that determine its performance. Therefore, even where each part is functioning effectively and efficiently, the system will not perform well as a whole. Furthermore, the component parts may lose their meaning and function when separated from the whole. A high performing system requires effective management of the interactions of its component parts [173]. 


\subsection{Complexity theory}

Complexity theory provides a useful framework for studying complex systems that are made up of sub-systems nested within a system with no central or external control. Outcomes from programmes or transformation initiatives in a complex adaptive system are non-linear and therefore a focus on interactions between component parts and the influence of contexts needs to be understood to understand how the system works. Complexity theory recognises that attempts to improve performance of a complex adaptive system requires LST initiatives that consider the core features of complex adaptive systems, seek to understand the effects of contexts and recognise the importance of being in the system rather than 'looking in' [11].

\subsection{Realist Research}

Westhorp [47] describes five key ideas in realism. The first idea is that anything with real effects is itself real, which means that social system and structures are real, programmes and policies are real, and institutional, social, cultural and political conditions have real effects on programme outcomes.

The second idea is that all reasoning and behaviours are shaped by our individual experiences and therefore it is difficult to have final truth or proof on whether, how and why programmes work. A realist approach allows researchers to work towards a closer understanding rather than the final truth.

The third idea asserts that all programmes are social systems and require a general understanding of systems theory. The component parts of the system interact and influence each other, and subsequently influence the programme outcomes.

The fourth idea proposes the concept of mechanisms that are underlying, unseen processes that exist in a system and influence the outcomes depending on the circumstances or contexts. Mechanisms cannot be observed using methods to determine programme outcomes. They exist as part of a whole system, are triggered by contextual factors, are explanatory, have empirical content and are testable $[47,174,175]$. Mechanisms can be both positive and negative and it is necessary to understand both in order to create the environment for an intervention to produce the desired outcomes.

The last idea focuses on the importance of context or circumstances. Contexts describe the organisational, social, cultural and political conditions that trigger the mechanisms, which then determine the programme outcomes $[47,175]$. Each context in which a programme is 
implemented, for example using a 'top-down' or a 'bottom-up' approach for LST initiatives, triggers a different set of mechanisms which then influence the outcomes. Context can be both an enabler and a barrier. A context that is enabling in one place can be a barrier in another owing to local needs and capability.

These five ideas culminate into forming mid-range theories in the form of context-mechanismoutcome (CMO) that explain what works for whom under what circumstances (contexts) and why.

Mid-range theories or programme theories, are essential for realist research [4]. They guide us to look for CMOs, in particular the mechanisms, as these are not directly observable [176]. Shearn, et al. [176] distinguished mid-range theory and programme theory based on how abstract and broad they are. They described programme theory as less abstract and highly specific concerning a specific intervention, while a mid-range theory is more abstract and broader and can be generalised for complex and large-system interventions.

The realist approach is becoming more common in health research and evaluation as it takes into consideration the contexts into which programme or interventions are introduced and explains why and how the intended or unintended programme outcomes emerged $[1,37,44$ $46,48,165,166,177-179]$.

Supporters of complexity theory argue that a realist approach to programme evaluations focusing on identifying the CMO configurations is too linear and does not recognise the multiple causation and non-linearity of complex adaptive systems [168]. The emergent, unpredictable and generative nature of the system defies the logic of such a linear approach. However, Westhorp [168] argues that "even complicated interventions boil down to simple mechanisms" (p 407). Pawson and Tilley's intent of a realist approach is not the linear identification of the CMO configurations but to study the underlying links between the intervention and the outcome, and the role of contexts and mechanisms when implementing change in a complex system $[4,170,171]$. Complexity theory argues that interactions between agents in complex adaptive systems generate internal rules that drive the actions and behaviours of the agents, which is fed back into the system and create further interactions (Figure 4.1) $[9,28,168]$. Both theories are therefore underpinned by multiple causation and non-linearity views that recognise the emergent, unpredictable and generative nature of complex adaptive systems and the important role of context in understanding the outcomes from a change effort. According to Westhorp [168], this similarity makes complexity theory and the realist approach "natural bedfellows" ( $p$ 
407). This similarity is used to situate this research as a realist approach nested within the framing of complex adaptive systems, as my initial programme theories on how the SLM programme worked (or not) give effect to the ideas of complexity theory.

\subsection{Evaluation versus research}

Chen [180] says that "the fundamental difference between evaluation and research lies in the purpose and motivation behind the work" (p 2).

An evaluation is typically commissioned by an organisation to judge the value or merit of a programme, and the results of an evaluation are used by programme directors and managers to inform their decisions on whether to continue to invest in that programme $[180,181]$. There is usually a contract between the agency funding the evaluation and the evaluators. An evaluation does not seek to discover new knowledge but to use existing knowledge to inform and guide practical action, i.e. to improve and not to prove [181]. The end product is a report that answers specific questions for a particular stakeholder such as the funding agency [180].

In contrast, research aims to answer an intellectual question and there is a greater emphasis on generating new knowledge, generalising findings and adding value to the research community $[180,181]$. Therefore, research outputs are shared widely through publication in journals, poster, abstract and conference presentations [180].

Both research and evaluation may use similar methods, theories and analysis approaches to answer their questions and to ensure the findings are reliable [180]. Based on the descriptions provided by Chen [180] and Clarke [181], I consider this study to be research study that is using a realist approach. I do not intend to judge the value or merit of the SLM programme or its future but to use the knowledge and experience gained from the implementation of the programme to inform the programme architecture for future LST initiatives in the New Zealand health system.

\subsection{The nested approach chosen for this research}

The realist approach, nested within the framing of complex adaptive systems, provided the overall logic of enquiry for this research (Figure 5.1: The nested approach to the research). This nested approach recognises the health system as a complex adaptive system, in which the emergent, unpredictable and generative nature of the system significantly influences the outcomes of any intervention or change $[34,139,140,166]$. 
Traditional realist evaluations focus on an intervention or a programme to find out 'what works, for whom, how, why and in what circumstances' and reveal the intervention-contextmechanism-outcome interplay $[4,170,171]$. They start with mid-range or programme theories formulated based on past experiences and previous research and end with refined theories that describe the context-mechanism-outcome interplay for the programme, initiative or the intervention [48].

In this research, I did not seek to evaluate the SLM programme to describe the contextmechanism-outcome interplay. Instead, I sought to build from my insights and experience of leading the implementation of the SLM programme and undertake a structured investigation to describe and analyse the programme architecture that underpins efforts to successfully implement LST initiatives in the New Zealand health system. I sought to use a theory building strategy proposed by Shearn, et al. [176] to build initial theories (referred to as initial propositions here onwards) to identify key elements that increases the chances of success with implementation of the SLM programme and how the contextual factors influences its success or failure.

The contexts in this research are the internal and external social and environmental conditions, such as the maturity of Alliances, quality of local relationships, the continuous improvement culture, and the leadership styles of the $\mathrm{MoH}$ and DHBs. These contexts, in the process of implementing LST initiatives such as the SLM programme, trigger mechanisms that affect the reasoning of health system leaders and health care professionals involved.

The mechanisms in this research are the underlying, unseen reasoning of health system leaders and heath care professionals prompted by the opportunities and resources available to them that determine their willingness and ability to support, lead and or be involved in the implementation of LST initiatives. These mechanisms influence the actions and behaviours of these leaders and health care professionals in the health system, which then influence the implementation of LST initiatives.

Given the uniqueness of the SLM programme, my motivation was to capture the knowledge and experience of those involved in leading and implementing the programme whose behaviours and insights are crucial in bridging the gap between the theory of LST initiatives and the reality of successfully implementing these initiatives in the New Zealand health system. 
Having observed from my implementation experience that the programme architecture that underpins efforts to successfully implement LST initiatives is poorly understood by the New Zealand health system leaders and Alliances, the intent of this research was to detail this architecture to increase the understanding of those leading and implementing LST initiatives. I hope findings from this research will be used by the New Zealand health system leaders and collaborative networks to enhance their capacity and capability for successfully implementing LST initiatives to achieve the desired system transformation. The research design that follows applies the five realist ideas introduced in this chapter. 


\subsection{RESEARCH DESIGN}

The research involved six phases as illustrated in

Figure 7.1. Three features of realist research - theory gleaning, refinement and consolidation, [49] - explain the sequence of the research. This sequence is an important tenet of realist research that involves using different perceptions to reveal the complexity of a system and refine the mid-range theories $[47,49,167]$. Realist research is an iterative process and therefore there was back and forth movement between the phases. Phase one (theory gleaning) informed phase two (literature review), which informed phases three (interviews) and four (workshops). Phases three and four occurred concurrently and subsequently informed phase five (survey). Phase six (maturity matrix testing) followed construction of the maturity matrix from phase four (workshops).

\subsection{The research setting}

The research was undertaken in the New Zealand health system as described in Appendix 1. I acknowledge that the New Zealand Health and Disability System Review, concluded at the time of writing this thesis, recommended changes to the structure of the New Zealand health system. However, regardless of how the health system is structured or funded, it will remain a complex adaptive system and will have sub-systems with macro, meso and micro component parts. Therefore, the research did not focus on the structural arrangements, funding model or contractual arrangements of the system or any of its components parts. However, how the current funding mechanisms are used to commission services, and how contextual factors help or hinder effective commissioning, were in scope.

The system agents or actors working in the health system that are involved in the implementation of the SLM programme are health care professionals, senior system leaders, middle managers, project/programme/portfolio managers, data analysts, and patient advocates or community representatives. The latter are involved through Alliances or DHB consumer councils. 


\section{Conceptual work}

Phase One: Initial architecture for successful implementation of LST initiatives revealed from insights and experience from the SLM programme. Architecture involves key elements, initial propositions on how elements work in different context, and understanding of elements and contexts

Use of complexity thinking to revise list of key elements and their descriptions

Literature review on maturity matrices as a way to enhance understanding of architecture for health system leaders and alliances

Analysis and consolidation of key elements and construction of CMOs that influence successful implementation of LST initiatives

Refined version of maturity matrix based on empirical work. Consolidation of CMOs that influence successful implementation of LST initiatives

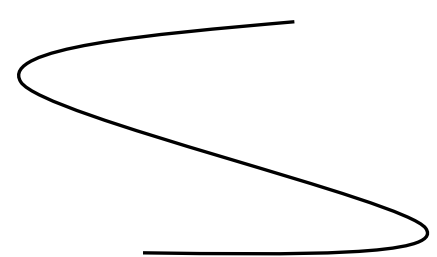

Phase Two: Review of grey and published literature to identify international evidence on key elements that support successful implementation of LST initiatives and how these elements work in different contexts
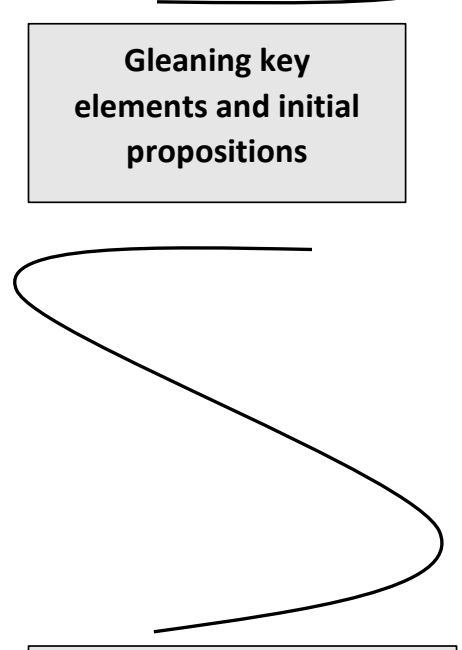

Refining key elements and initial propositions, and first draft of maturity matrix

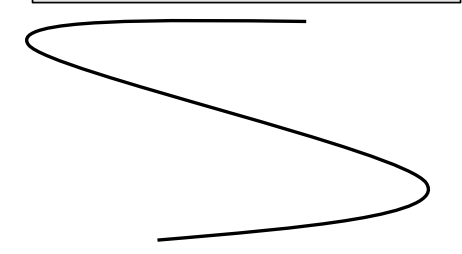

Consolidation of key elements and refined initial propositions

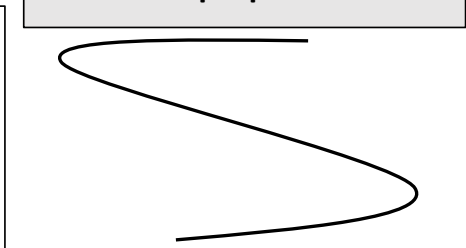

Phase Three: Interviews with key stakeholders from New Zealand and international health system ( $n=9)$ and local stakeholders from nonhealth sector $(n=3)$ to test key elements and to investigate how these work in different contexts to influence successful implementation of LST initiatives

Phase Four: Two workshops with same key stakeholders from the NZ health system $(n=10)$ : first to test key elements and to investigate how these work in different contexts; and second to construct a maturity matrix to increase understanding of architecture for successful implementation of LST initiatives

Phase Five: Online survey with frontline health professionals and managers involved in the implementation of the SLM programme to consolidate key elements, further refine initial propositions that influence successful implementation of LST initiatives, and explore CMOs for the SLM programme $(n=51)$

Phase Six: Field testing of maturity matrix in $\operatorname{ALTs}(n=3)$ and review of maturity matrix by MoH Māori Strategy and Policy team

\section{Programme architecture that includes maturity matrix for key elements and CMO theories that influence successful implementation of LST initiatives}




\subsection{Data collection and analysis}

Data collection for the research corresponded to the six research phases (

Figure 7.1) and analysis often occurred simultaneously and informed subsequent phases.

Three data collection strategies were used [173] taking into consideration Westhorp's [47] five ideas of realism described in Chapter 6 (Realist research). First, my knowledge about the complexity of the health system enabled me to understand the contractual, funding and policy instruments present in the New Zealand health system that enable and constrain LST initiatives. This understanding was important for building initial propositions about how the prevailing structures, behaviours and processes influenced the implementation of the SLM programme. This understanding also determined my initial list of key elements and shaped the design of my workshops, and the interview and the survey questions.

Second, personal experience, engagement and observations allowed me to use insights, experiences, and relationships to select the right participants for the research to identify and understand the programme architecture of LST initiatives. These participants whose reasoning I was seeking to understand were those charged with making major strategic decisions about where effort goes towards supporting implementation of LST initiatives. Much about the SLM programme and the knowledge and experience of its development and implementation at the district level is not published and remains in the heads of individuals working on the programme. Most of the people who were involved in the original concept and design of the SLM programme are still working in the health system and are in senior leadership roles. I wanted to use the knowledge and experience of those working in the system and those leading or involved in the implementation of the SLM programme, to inform the answers to my research questions so I could more explicitly reveal the programme architecture for successful implementation of LST initiatives. These experiences were important to elicit the reasoning and behaviours (mechanisms) triggered by organisational, social, cultural and political contexts that influence the successful implementation of LST initiatives.

Third, qualitative data was collected from a review of relevant literature, interviews, interactive workshops and the survey. These yielded rich descriptions of contexts, mechanisms and outcomes to form mid-range theories and gain a closer understanding of the programme architecture for LST initiatives. 
Analysis of data collected was guided by five key strategies: (i) respecting and capturing individual perspectives and experiences; (ii) deductive technique to identify key elements and an inductive technique to construct the maturity matrix; (iii) a systems mind set taking into consideration the system dynamic that is not limited to a linear cause-and-effect relationships; (iv) retroduction to identify the underlying mechanisms; and ( $v$ ) reflexivity with my voice as a researcher and being authentic and balanced with my perspective $[173,183]$.

An inductive technique builds theory from multiple observations; a deductive technique starts from theory and tests propositions to confirm, refute and refine [183]. Retroduction is used in realist research and evaluations to identify the underlying, unseen mechanisms that explain why programmes or interventions work (or not) in given contexts [183]. Retroduction uses both inductive and deductive logic, and requires that inquirers use their insights, hunches, common sense, intelligence and expertise to build and test theories about the underlying, unseen mechanisms [183]. Sections $7.3-7.8$ describe each research phase and strategy in detail.

While being inside the system extended me the privileges of insights, experiences and relationships, it also created real and perceived bias. How I managed this bias is discussed in chapter 8.

\subsection{Phase one: Theory gleaning}

The aim of Phase one was to outline the programme architecture for successful implementation of the SLM programme using my own insights and knowledge. I took into consideration the realist ideas that gleaning this architecture would require the use of complex adaptive system ideas (i.e. the idea that any change is likely to be conditional on the contexts it is implemented in). I recognised that the health system structure and the interaction of its agents affected the outcomes of the programme and paid attention to mechanisms triggered by organisational, social and political contexts. I started the gleaning process by identifying a list of key elements that supported the implementation of the SLM programme. I then developed initial propositions on how these elements worked in different contexts to influence the successful implementation of the SLM programme.

I used two data collection strategies for this phase of the research: documenting my first-hand insights and knowledge gained through leading three annual cycles of the SLM programme implementation; and informal conversations with the programme clinical lead and those involved in the implementation of the SLM programme. Phase one had begun approximately 12 
months prior to my formal enrolment into the doctoral programme. These early insights were the premise of my doctoral research proposal. My hypotheses on the key elements and the initial propositions were tested in the later research phases.

\subsection{Phase two: Literature review}

A realist approach involves iterative searching of the literature, rather than using a fixed protocol, beginning with a broad direction, and refining progressively. This enables the research to be flexible and respond to emerging findings $[4,170,171]$. Therefore, an iterative review of published and grey literature was undertaken at all research phases.

Phase two had three aims. First, it sought to identify international evidence on the key elements that support the successful implementation of LST initiatives and improve the performance of health systems. Second, it sought to understand how these elements work in different contexts to influence the success of LST initiatives. Third, it sought to identify an outline for the selfassessment maturity matrix that participants in phase four (workshops) would use to construct a tool to support a learning system.

There were five stages of literature review in the research. Stage one involved identifying published and grey literature on high performing health systems. The published literature was searched using keywords in the OVID and PUBMED databases. At this stage of the research, I did not have a definition for LST initiatives, so a broad range of keywords were used to search literature relating to performance, governance, accountability, transformation and measuring quality improvement. The search was limited to English language from 2008 to 2018. Key words used to guide the search were: health system, state medicine, national health programmes, primary health care, health services accessibility, continuity of patient care, quality of health care, delivery of health care, efficiency, organisational, financing, governance, measuring for health performance, health accountability.

Stage two was to identify national and international studies and grey literature on systems and complexity theories and their application in health systems world-wide and LST initiatives in the health system. The published literature was searched using keywords in the OVID and PUBMED databases. Key words used to guide the search were: high performing system, large system, large scale (improve, system, transform, change), state medicine, national health programmes, systems theory, complex adaptive, systems thinking, complexity thinking, and complexity theory. 
Grey literature was searched using Google and visiting known quality improvement websites in the United States, United Kingdom, Canada, Australia, and New Zealand. These included websites such as the King's Fund, Nuffield Trust, Institute of Healthcare Improvement, Health Quality \& Safety Commission, World Health Organization, and the Commonwealth Fund. The search was refined to identify studies relating to LST initiatives in health system at macro or meso level available in English from 1946 to 2018.

Stage three of the literature review was used to identify published LST initiatives in sectors other than health. Keywords used in OVID and PUBMED databases, in addition to stage two, were: other industry or industries, oil or gas, energy, aviation, construction, automobile, Toyota and lean.

Stage four of the literature review related to maturity matrices. The published literature was searched using keywords in the OVID and PUBMED databases using the keywords: selfassessment and performance. The search was limited to English language from 1946 to 2018.

Stage five of the review was a continuous snowball approach to identify further relevant published and grey literature. This included checking reference lists of previously identified literature, reviewing citation lists for material I had, reviewing newsletters from research institutes and receiving literature identified by the research supervisors, SLM programme clinical lead, research participants and research colleagues. This stage continued to the end of the research period.

The evidence from this phase was used to refine the list of key elements that support successful implementation of LST initiatives and to further identify contexts and mechanisms that influence the successful implementation of these initiatives. Phases one and two informed phases three and four of the research.

\subsection{Phase three: Interviews}

Interviews are the most commonly used method to collect data in realist research [49]. Data collection for interviews is based on the premise that the researcher's initial theories are the unit of analysis and information elicited from the interviewee confirms, rejects or refines the theories [4]. This relationship is described as a teacher-learner cycle in which the interviewer starts as a teacher explaining the theory to the interviewee, and then the interviewee having learnt the theory, starts teaching the interviewer about the CMOs for the programme based on their experiences $[49,174]$. 
Therefore, it is essential that the researcher is embedded in the complexity of the system to learn and experience as theories emerge rather than looking in [173].

The aim of the interviews was to examine the programme architecture of LST initiatives by testing key elements that support successful LST initiatives and investigating how these elements work in different contexts to influence these initiatives.

The interviews in phase three followed realist guidance in terms of sampling, participant selection, question design and interview technique [49].

\subsubsection{Selection of participants}

Pawson and Tilly advise that prior knowledge of the researcher can assist in the selection of interviewees, enables framing of the interview questions, and builds rapport with the interviewees to positively influence the interview dynamics to consolidate the CMO configurations during the interview $[4,167]$.

I used purposeful sampling technique to recruit participants for the interviews. Key informants were identified strategically at first; and then opportunistically from informal conversations with sector leaders. The aim was to identify participants that were 'information rich' and could provide a view on leading LST initiatives in a complex system, both in health and non-health sectors. From a health system perspective, participants were identified using the following criteria:

- $\quad$ Experience of working in the health system in New Zealand in a senior role with Māori perspective and leading LST initiatives.

- $\quad$ Experience of working in the health system overseas in a senior role leading LST initiatives. Other countries identified were Australia, Canada, Scotland, and Europe.

- Those currently not working in the health system in New Zealand but have the knowledge of the system structure and how it influences implementation of LST initiatives from research and academic perspective.

- Those who are working in New Zealand health system as senior consultants providing expert advice to DHBs and the MoH on system performance, national policy and LST initiatives.

Given the limited number of interviews planned, I focussed on recruiting senior system leaders for this research phase. These leaders are usually in charge of initiating and supporting the implementation of LST initiatives and making decisions relating to funding and resource allocations. While involvement of frontline staff is critical for 'bottom-up' innovations, support 
of senior system leaders is also critical for sustainability, scaling-up and spreading of these innovations in a complex adaptive system. I planned to use an online survey to gather data from middle management and frontline staff (phase five).

Initially 12 participants were identified through personal and professional contacts in discussion with my supervisors. Further five participants were identified as the data collection phase progressed through informal conversations with senior leaders in health system. Participants from the research community were identified based on their academic publications on complexity thinking and system transformation in the health sector in New Zealand and internationally. This was a useful approach to identify participants from health systems overseas.

Following initial ethics approval, I emailed the participants with the overview of the research including a participant research information sheet (Appendix 3), the consent form (Appendix 4) and the research information sheet and consent form for Chief Executive (Appendix 5). Some of the participants, who were company partners, directors, or independent contractors or clinicians found it irrelevant or impractical to seek Chief Executive consent. I sought an amendment from the ethics committee for an exemption for these participants to submit Chief Executive consent, which was granted.

Key informants from outside the health sector were recruited to obtain perspectives from other complex systems. Following discussions with my supervisors, industries of interest identified were banking, construction, and organisational transformation. For non-health sector participants, I used my personal and professional contacts to identify potential participants with the right level of experience on system transformation and seniority in the organisation. These interviews were conducted after the two workshops in phase four. I sent potential participants the key elements identified from the workshops with descriptions along with the research information sheet (Appendix 3) and consent form for them (Appendix 4) and their Chief Executives (Appendix 5). This provided the participants with additional information to decide if they could constructively contribute to the study.

My aim was to interview 10 participants. I invited the initial 12 participants, and then went on to invite a further five as the interview phase progressed. Of the 17 participants invited, three did not respond and two declined to participate, one owing to work demands while the other did not think they had the relevant experience to contribute to the study. The five participants who did not participate were health consultants in New Zealand $(n=2)$ and overseas health researchers $(n=3)$. 
The 12 participants who completed the interview fulfilled my sampling criteria. Table 7.1 shows the profile of the interview participants and the code assigned that is used in the chapters ahead to attribute their contributions.

\begin{tabular}{|c|c|c|}
\hline $\begin{array}{l}\text { Interview } \\
\text { code }\end{array}$ & Profile & Affiliation \\
\hline IP01 & $\begin{array}{l}\text { Health consultant and involved in the } \\
\text { development of the SLM programme }\end{array}$ & Health consultancy in New Zealand \\
\hline IP02 & Academic and health researcher & $\begin{array}{l}\text { Health researcher in a New Zealand } \\
\text { University }\end{array}$ \\
\hline IP03 & $\begin{array}{l}\text { Health consultant and previous Chief Executive } \\
\text { of a DHB }\end{array}$ & Health consultancy in New Zealand \\
\hline IP04 & $\begin{array}{l}\text { Health consultant and direct experience in the } \\
\text { New Zealand health system }\end{array}$ & Health consultancy in Europe \\
\hline IP05 & $\begin{array}{l}\text { Māori clinician and involved in the } \\
\text { development of the SLM programme }\end{array}$ & Health researcher in New Zealand \\
\hline IP06 & $\begin{array}{l}\text { Clinician and leader of an LST initiative in } \\
\text { Scotland }\end{array}$ & National Health Service (NHS) Scotland \\
\hline IP07 & $\begin{array}{l}\text { Health consultant and direct experience in the } \\
\text { New Zealand health system and NHS }\end{array}$ & Health consultancy in New Zealand \\
\hline IP08 & $\begin{array}{l}\text { Health researcher of LST initiatives in complex } \\
\text { adaptive systems }\end{array}$ & Health consultancy in Canada \\
\hline IP09 & $\begin{array}{l}\text { Clinician and leader of an LST initiative in } \\
\text { Australia }\end{array}$ & Health researcher in Australia \\
\hline IP10 & Managing director & Construction company in New Zealand \\
\hline IP11 & Technology and risk manager & New Zealand Bank \\
\hline IP12 & Strategic management consultant & $\begin{array}{l}\text { Private consulting - New Zealand, } \\
\text { Australia and United Kingdom }\end{array}$ \\
\hline
\end{tabular}

Table 7.1: Interviewees profile and codification

\subsubsection{Interview process}

The date and time for each interview was confirmed with each participant once they had returned their consent form. Interviews were arranged either face-to-face, via telephone or via zoom meeting technology. Interviews ranged from 45 to 70 minutes and were audio recorded, which were later transcribed by a professional transcriber. I listened to audio files to check the accuracy of the transcripts and corrected any errors. The transcripts were then shared with those participants who had requested this through their consent forms to check and amend. Participants were given two weeks to return their amended transcripts to me.

I used seven pre-determined questions ranging from semi-structured, structured prompts and open-ended conversations. Interviews were designed and conducted using the guide published 
by Manzano [49]. Table 7.2 shows how the questions were designed in accordance with the guide.

The interview started with introduction of the participant and their area of expertise. I used the teacher-learner method, beginning as a teacher to introduce my initial propositions and evidence from the literature. I then switched to learner and sought participants' perspective. This method was used throughout the interview process. As the interviews progressed, in addition to the predetermined questions, I started to test perspectives and theories discovered from earlier interviews. This is true to the realist methodology that enables the researcher to refine and refute theories [49].

\subsubsection{Data analysis}

Data analysis computer software programs are generally not suitable or essential in realist research because data analysis is not a technical process once the research is completed but is an iterative process of discovering 'nuggets of evidence' throughout the data collection process $[49,167]$. I did, however, use NVivo computer software programme to aid thematic analysis of the interview transcripts. I generated codes for the list of elements that were tested; additional elements that were identified by the participants; and CMO theories identified by the participants. Transcripts of the interviews were coded deductively for key elements, and inductively to capture additional elements and CMO theories. Direct quotes from the transcripts were also used with all identifying information removed to protect participants' anonymity. 
1. Can you tell me little bit about your area of expertise?

2. My understanding of complex adaptive systems is that these are open systems with blurred boundaries and unpredicted ways of workings, whose agents learn, interact and adapt their behaviours to a changing environment.

Can you tell me your understanding of complex adaptive systems and how it relates to the (health system or your sector)?

3. Literature shows that in order to effect change in a complex adaptive system, LST initiatives are required. By LST initiatives I am referring to co-ordinated, system-wide change involving multiple organisations that takes into consideration the relationships, interactions and behaviours of those in the system. To what extent do you agree or disagree with this?

\begin{tabular}{|c|c|c|c|}
\hline $\begin{array}{l}\text { Strongly } \\
\text { disagree }\end{array}$ & Disagre & Neutra & Agree \\
\hline
\end{tabular}

4. Insights from the System Level Measures programme and evidence from literature tells me the following elements are necessary to implement LST initiatives successfully and sustainably.

To what extend do you agree or disagree with each of these?

\subsection{Alliancing way of working}

Alliancing is a clinically led, community engaged forum that brings different component parts of the health system together with the aim of transforming services so that they are best for the patient and best for the system. Alliancing is a way of working, reliant on trusted relationships and supported by enablers critical to continuous improvement. Successful Alliances bring alignment to multiple organisations and services present in a complex system, to achieve a common set of patient-centred goals. Alliancing is not about managing finances or organisational or professional accountabilities.

\begin{tabular}{|l|c|c|c|c|}
$\begin{array}{c}\text { Strongly } \\
\text { disagree }\end{array}$ & Disagree & Neutral & Agree & $\begin{array}{c}\text { Strongly } \\
\text { Agree }\end{array}$ \\
\hline
\end{tabular}

4.2 Clinical leadership and engagement

\begin{tabular}{|c|c|c|c|c|}
\hline $\begin{array}{c}\text { Strongly } \\
\text { disagree }\end{array}$ & Disagree & Neutral & Agree & $\begin{array}{c}\text { Strongly } \\
\text { Agree }\end{array}$ \\
\hline
\end{tabular}

\subsection{Use of commissioning cycle}

Commissioning is the process of continuously developing services and committing resources to enable the best health outcomes to be achieved for individuals and the population, equity to be assured and experience enhanced within the resources available (Productivity Commission NZ).

\begin{tabular}{|l|l|l|l|l|}
$\begin{array}{l}\text { Strongly } \\
\text { disagree }\end{array}$ & Disagree & Neutral & Agree & $\begin{array}{c}\text { Strongly } \\
\text { Agree }\end{array}$ \\
\hline
\end{tabular}

Rapport building - to get them talking

Introduce the concept of systems thinking as a teacher

Switch to learner

Introduce LST initiatives as a teacher

Switch to learner and use prompt guide to refine theory

Identify key elements. Start as a teacher outlining each theory and then switch to learner and use prompt guide to confirm or refute each theory. At the end look for additional information which may refine theory or introduce new ones. 
4.4 Integrated health information

\begin{tabular}{|l|c|c|c|c|}
\hline $\begin{array}{c}\text { Strongly } \\
\text { disagree }\end{array}$ & Disagree & Neutral & Agree & $\begin{array}{c}\text { Strongly } \\
\text { Agree }\end{array}$ \\
\hline
\end{tabular}

4.5 Analytic capability

\begin{tabular}{|l|l|l|l|l}
$\begin{array}{l}\text { Strongly } \\
\text { disagree }\end{array}$ & Disagree & Neutral & Agree & $\begin{array}{c}\text { Strongly } \\
\text { Agree }\end{array}$ \\
\hline
\end{tabular}

4.6 Continuous quality improvement focus

\begin{tabular}{|l|l|l|l|l|}
\hline $\begin{array}{l}\text { Strongly } \\
\text { disagree }\end{array}$ & Disagree & Neutral & Agree & $\begin{array}{c}\text { Strongly } \\
\text { Agree }\end{array}$ \\
\hline
\end{tabular}

4.7 Engagement with patients and communities

\begin{tabular}{|l|l|l|l|l|}
\hline $\begin{array}{c}\text { Strongly } \\
\text { disagree }\end{array}$ & Disagree & Neutral & Agree & $\begin{array}{c}\text { Strongly } \\
\text { Agree }\end{array}$ \\
\hline
\end{tabular}

Do you have anything else to add to this list?

5. For the next set of questions, I would like to explore contextual factors that influence the key elements and what impact these have.

Let's choose your top three key elements from the list above. What local circumstances, at the organisational level, influence these elements and in what way? What causes these effects? Give me an example

6. What national, social or political circumstances influence your top three key elements and in what way? What causes these effects? Give me an example

Exploring organisational contexts, mechanisms and outcomes

Conversational technique switch between teacher and learner during the conversation depending on the interviewee engagement. May test theories discovered in earlier research phases

Exploring national contexts, mechanisms and outcomes Conversational technique switch between teacher and learner during the conversation depending on the interviewee engagement. May test theories discovered in earlier research phases

Concluding question

End the interview as a learner capability for improvement that will enable successful and sustainable implementation of LST initiatives in the health system? 


\subsection{Phase four: Workshops}

The aim of the workshops was to use the knowledge of those working in the health system to inform the programme architecture for successful implementation of LST initiatives in three ways: to refine the key elements needed to support the successful implementation of LST initiatives; to identify the contexts and mechanisms that influence these initiatives; and to construct a maturity matrix to increase the health system leaders' and Alliances' understanding of this architecture. The selection of participants for the workshop followed realist guidance inviting people who were involved in the design or the implementation of the SLM programme and those who had significant experience and knowledge about the programme [49].

The $\mathrm{MoH}$ had used interactive workshops to co-design the SLM programme with the health sector. My experience was that these workshops were an effective method to bring key people together and using a facilitator, explore their experiences and knowledge, stimulate creative thinking and reach consensus on key decisions in a short period of time through collaborative working. This ensured that the output was suitable for the New Zealand health system and had a wider sector buy-in. This collaborative policy design process was a unique feature of the SLM programme; usually national policies are designed by the $\mathrm{MoH}$ on behalf of the government of the day.

The two workshops in phase four were designed and conducted using the guidance from Pavelin, et al. [184].

As part of workshop planning, I considered the venue, both for ethical reasons to mitigate perceived or real conflict of interests with my dual role, and the type of room that would allow creative thinking. For these reasons, the workshops were held in the Rutherford House at the Pipitea Campus of the University. The University environment reinforced the separation of my role as a researcher from that of $\mathrm{my} \mathrm{MoH}$ role. The building is centrally located and was easy for participants to get to. The rooms chosen had large windows that allowed natural light and provided the floor and wall space for group activities. The two workshop dates, 21 and 28 August 2019, were determined based on the availability of appropriate rooms and the interval between the workshops was also considered to maintain momentum of the group and maximise chances of successful outcome. 
Other important considerations were choice of facilitation activities that would yield the information to answer the research questions and a facilitator who was impartial but had relevant subject matter expertise and credibility with the participants given their seniority [184]. I chose two facilitation activities that I had prior experience: 'storm and sort' [185] and 'stay and stray' [186]. These two techniques were used in the first workshop, details of which are discussed below.

Following discussions with my supervisors, I asked the SLM programme clinical lead to facilitate the workshops. The SLM programme clinical lead had led the co-development of the SLM programme and is a practicing-clinician in the sector and therefore had the relevant skills, subject matter expertise and credibility to elicit information from participants about what worked and what did not work with the implementation of the SLM programme.

I provided refreshments for participants that included a coffee tab at the café in the building and catered lunch. This was an important part of showing hospitality and keeping the participants nourished throughout the day to maintain energy levels. Having the lunch catered also meant that participants could stay at the venue and continue to network with fellow participants, which most of them did. The Victoria University Faculty Grant was used to provide catering for the workshops.

In planning the agenda for the workshops, I considered three phases: opening, exploring and closing; resources required such as butchers paper, post-it notes, markers and pens; audio-visual equipment for presentations and giving group activity instructions; data collection methods that included pre-outlined templates; and audio recording of group sessions [184]. I briefed the facilitator prior to the workshops so they were clear on tasks, processes and outcomes of each session and had debrief following the first workshop to identify any areas for improvement for the second workshop.

\subsubsection{Workshop participants}

Using a purposeful sampling method, participants for the workshop were recruited based on their role and experience in the New Zealand health system. Participants were senior leaders and clinicians who are responsible for leading LST initiatives or those charged with making major strategic decisions about where effort goes towards supporting these initiatives in their organisations. Some of the participants were involved in developing the original concept of the SLM programme in 2012 to 2015 , others were leading or involved in the implementation of the 
SLM programme in their districts. Other variables that were considered in the selection of the participants were: recognised leaders in the health system; Māori perspective; equity perspective, in particular for Pacific population; advocates of service users who understood the complexity of the health system; rural perspective; and nursing and allied health perspectives.

A list of potential participants was identified from those known to me through my working experience in the health sector, and in discussion with my supervisors and the SLM programme clinical lead. Some of the participants were also identified through conversations and presentations of the research proposal to sector groups, such as the PHO clinical leaders' forum, DHB General Managers planning and funding, and the PHO Chief Executives' group. An attempt was made to invite a senior clinical or operational leader from the DHB or PHOs of each district. The broad sampling ensured inclusion of participants with a wide range of views to manage the facilitator's and my (unconscious) bias. Given the seniority of participants and the two-day commitment, a large sample of participants were invited with the aim of having 10-12 participants who could attend both workshops.

Thirty-three participants were invited, via email, to participate in the two workshops. The email included the participant information sheet (Appendix 3) and consent form (Appendix 6). Participants were asked to confirm their availability and willingness to participate in the workshops. The email advised participants that written consent would be needed from the Chief Executive of their employing organisations and that if they were willing and available to participate, they brief their Chief Executive accordingly. Once participants confirmed their willingness and availability to participate, I then emailed their Chief Executives the research information and consent form (Appendix 5). Participation in the workshop was confirmed once I received consent forms from the participant and their Chief Executive. There were some participants who were either self-employed or worked as independent contractors or clinicians, making it impractical to seek Chief Executive consent. I sought an amendment from the ethics committee for an exemption for these participants to submit Chief Executive consent, which was granted.

The Victoria University Faculty Grant was available to support participants who wanted to participate but did not have financial support of their organisation or where they were independently employed and needed to personally fund the cost to attend the workshops. Eventually, the grant was used to support travel cost of one participant who was a privately employed clinician. For rest of the participants, the Chief Executives of their employing 
organisation agreed to pay travel costs and allowed them to attend the workshop during business hours.

Figure 7.2 shows the details of participants invited, numbers that accepted and declined, and the attendance at the two workshops.

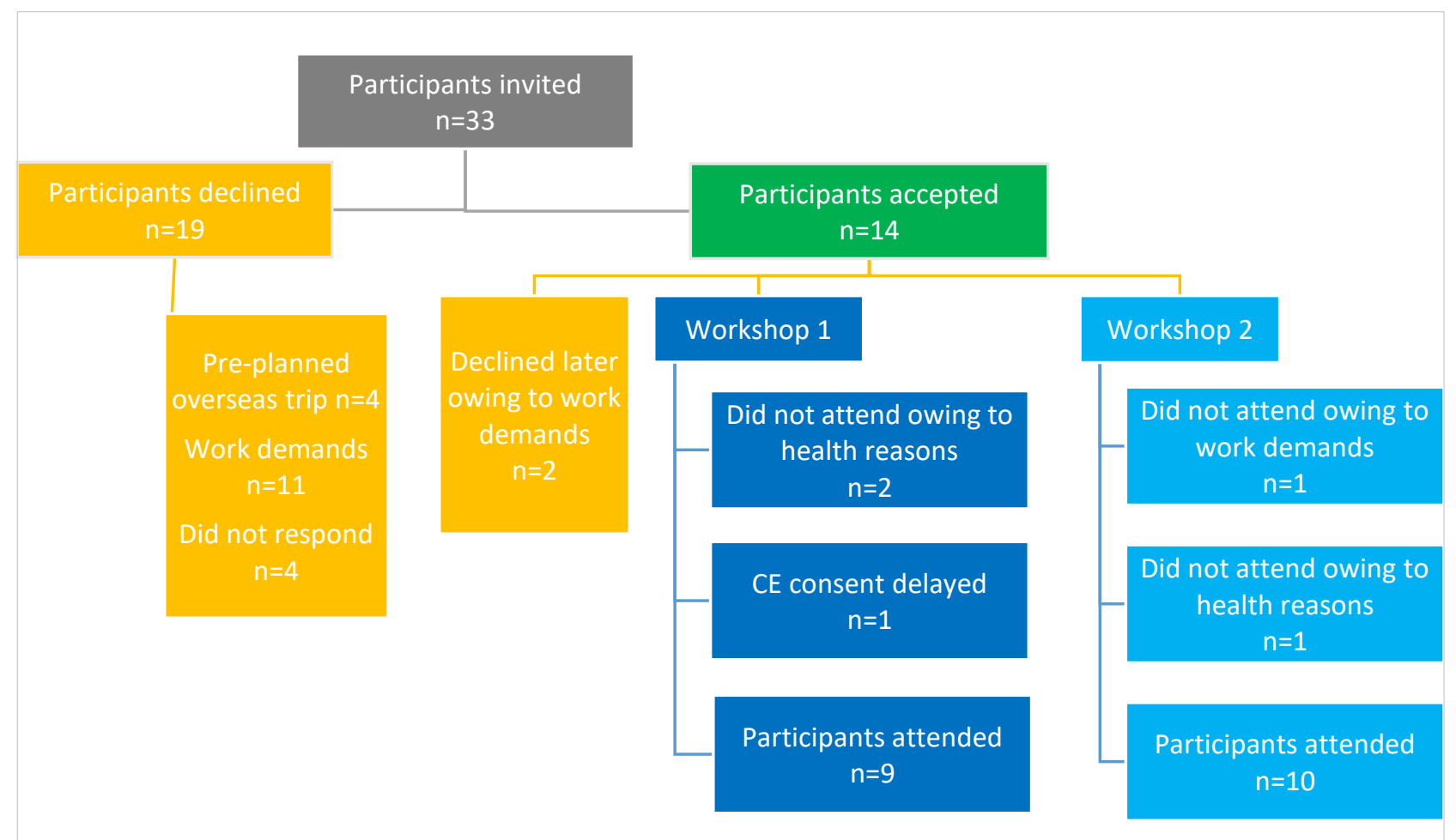

Figure 7.2: Workshop participants

\subsubsection{Workshop one}

I sent the agenda of the workshop to participants two days prior (Appendix 7). Of 12 participants who had accepted, nine attended the workshop. Of the three participants that did not attend, two were owing to health reasons and the third did not receive their Chief Executive consent in time.

The workshop started at 09:30 hours with the facilitator opening the session by welcoming participants and thanking for their attendance. The facilitator then asked participants to introduce themselves, which included participants' name, place of work, and their reason for attending the workshop. This session was audio recorded and later transcribed by a professional transcriber.

Following introductions, I presented an overview of the research that included my motivation for the research; key New Zealand and international literature findings; initial propositions gleaned 
from the SLM programme; and the research questions, methodology, timeline, and key outputs. Doing so set the scene for the workshop and brought all participants to a common understanding and starting point.

Participants were reminded of their rights as a participant, how the information they provided was going to be used and how I was managing any real or perceived conflicts of interest with my dual role. Participants were also given some ground rules for the workshop that included:

- $\quad$ meeting confidentiality and respecting other participants' anonymity

- $\quad$ that while participants did not need to agree with others, they needed to be respectful in listening to different perspectives

- $\quad$ that there were no right or wrong answers, and that individual experience and opinions were important

- $\quad$ that parts of the workshop would be audio recorded.

The room was set up with three tables in an arc facing the front of the room so all participants could face the facilitator and the television screen. At each table, an information pack that contained the agenda for the day, a summary of literature findings on the key elements that support LST initiatives, copy of the research presentation, and data collection template for individual ratings of the key elements. The literature summary included:

- Findings from eight international studies (in a table format) with reference, study aims and setting, and the key elements identified as necessary to support LST initiatives.

- Insights from the SLM programme and the literature review on the key elements that supported the implementation of LST initiatives and definitions of these.

- From the interviews completed prior to the workshop, the local and national contexts that influenced implementation of LST initiatives, the effect (outcomes) and the causes (mechanisms).

Along with the information pack, pens, markers, and post-it notes were also provided.

The workshop contained three sessions to build a detailed picture of the programme architecture that underpins efforts to successfully implement LST initiatives. My role in the workshop was a participant observer. I provided clarifications (when asked) and assisted the facilitator with collection and real-time analysis of data. 


\section{Session one - Consensus on key elements}

Session one was used to reach consensus on the key elements that support LST initiatives using the 'storm and sort' activity. This occurred in five rounds.

Use of this activity allowed the participants to review the literature summary, consider the list of key elements provided, and individually reach a decision before discussing as a group. This meant that all participants had equal opportunity to contribute with their own perspective and not be influenced by a dominant voice at the table.

First, participants were asked to individually review the literature summary and using the data collection template provided (outline shown in Table 7.3), record to what extent they agreed or disagreed with the initial set of the key elements provided and identify further elements that they perceived as important, either from their experience or literature summary.

Second, at the table in groups of three, participants were asked to select the key elements that all three group members either agreed or strongly agreed with.

Third, at the table in groups of three, participants were asked to select the key elements that at least two members either agreed or strongly agreed with. Pre-outlined butchers paper was provided for each table to record findings from second and third rounds.

The groups were then asked to discuss their findings and reach consensus on a list of key elements. During this time, I visited each table to record the additional elements identified on my laptop and projected this on the large television screen.

Fourth, the groups of three participants at each table were asked to repeat the second and third steps of the 'storm and sort' activity for the additional elements identified. 
Alliancing way of working

\begin{tabular}{|c|c|c|c|c|}
\hline Strongly disagree & Disagree & Neutral & Agree & Strongly Agree \\
\hline \multicolumn{5}{|c|}{ Clinical leadership and engagement } \\
\hline Strongly disagree & Disagree & Neutral & Agree & Strongly Agree \\
\hline \multicolumn{5}{|c|}{ Use of commissioning cycle } \\
\hline Strongly disagree & Disagree & Neutral & Agree & Strongly Agree \\
\hline \multicolumn{5}{|c|}{ Integrated health information } \\
\hline Strongly disagree & Disagree & Neutral & Agree & Strongly Agree \\
\hline \multicolumn{5}{|c|}{ Analytic capability } \\
\hline Strongly disagree & Disagree & Neutral & Agree & Strongly Agree \\
\hline
\end{tabular}

Continuous quality improvement focus

\begin{tabular}{|l|c|c|c|c|}
\hline Strongly disagree & Disagree & Neutral & Agree & Strongly Agree \\
\hline
\end{tabular}

Engagement with patients and communities

\begin{tabular}{|l|l|l|l|l|}
\hline Strongly disagree & Disagree & Neutral & Agree & Strongly Agree \\
\hline
\end{tabular}

\section{Do you have anything else to add to this list?}

Table 7.3: Individual rating of key elements

Lastly, the facilitator repeated the second and third steps of the 'storm and sort' activity at the overall group level. A group discussion followed, which led to a list of key elements to support successful implementation of LST initiatives in the New Zealand health system that all participants either agreed or strongly agreed with. This session was audio recorded and transcribed later by a professional transcriber. I took notes in my notebook.

\section{Session two - Key element definitions and outcome descriptors}

Session two defined and described the outcomes for the key elements identified in session one and used the 'storm and sort' technique and the 'stay and stray' technique.

First, participants were asked to individually consider the list of elements identified in session one and using post-it notes or pen or marker, write couple of sentences describing the element and what would the established element demonstrate, that is, what does good look like (outcome descriptor).

Second, participants were asked to share and discuss their individual perspectives. 
Third, participants were asked to reach consensus on the definitions and outcome descriptors among three participants at their table. Where consensus could not be reached, the groups were advised to include all potential definitions and outcome descriptors. Pre-outlined butchers paper was used to record the findings.

Next the 'stay and stray' activity was used to move participants around other tables so they could gather a sense of other groups' work. This involved one person from the group staying at the table to explain and answer questions, while the other two participants moved around. The activity was repeated so all three groups had seen other groups' work. Participants then returned to their original table to review their findings and amend their work. I collected the butcher paper and transcribed the information in the days following the workshop.

\section{Session three - Identifying contexts, mechanisms and outcomes}

Session three explored contexts and mechanisms that influenced the successful implementation of LST initiatives and used the 'storm and sort' technique and the 'stay and stray' technique.

First, participants were asked to individually consider the local and national circumstances that influenced the key elements, its outcomes and mechanisms that caused the outcomes. Participants recorded this using Post-it notes. Second, participants were asked to discuss at their table and record their findings on the butcher paper

Next the 'stay and stray' activity was used to move participants around other tables so they could gather a sense of other groups' work. I collected the butcher paper and transcribed the information in the days following the workshop.

The session concluded with an overall group discussion on the local and national contextual factors that influence successful implementation of LST initiatives, and the causes and the effects of these factors. This session was audio recorded and transcribed later by a professional transcriber.

At the end of the workshop, I agreed to share with the group, prior to the second workshop:

- The key elements agreed by the group; the definitions; outcome descriptors; and contexts and mechanisms that influence successful implementation of LST initiatives.

- Electronic copies of the literature summary and the research presentation.

- The outline of the maturity matrix that participants would be constructing in the second workshop. 
- A hyper-link to an European maturity model for integrated care [163].

The workshop finished at 15:45 hours. Once the participants left the room, the facilitator and I reflected on the workshop, in particular, what went well and what we could do differently in the second workshop for improvement.

Following the workshop, I got in touch with the three participants that did not attend and offered to catch up with them before the start of the second workshop and brief them on the outputs of the first one. Two of the participants responded and said that they would like to read the notes of the first workshop and would contact me if they needed a follow up conversation. They were sent the notes, but they did not ask for a follow conversation. The third participant did not respond.

\subsubsection{Data analysis}

Data from the workshop was analysed in three stages. First, it was analysed in real-time during the first session of the workshop using a laptop that was projected on the television screen. This method enabled the facilitator to conduct an iterative process until consensus was reached on the key elements. I assisted the facilitator with the real-time analysis by electronically capturing data during the 'storm and sort' activities. Data from the first session informed the subsequent sessions of the first workshop.

Second, following the first workshop, I tabulated participants' individual ratings on the initial set of key elements that was shared with participants at the workshop from the A3 data collection templates (Table 7.3) completed at the beginning of the first session. I used the data collected using butchers paper and audio recording to collate outcome descriptors for the key elements. The CMO configurations were constructed using the data collected on the butcher paper, my notebook and audio recordings. Data collected from the first workshop informed the construction of the maturity matrix in the second workshop.

The third stage was following both workshops, which is discussed later.

\subsubsection{Workshop two}

The aim of the second workshop was to construct the maturity matrix using the experience and knowledge of those working in the New Zealand health system. Participants had an opportunity to review an European maturity model for integrated care [163] prior to the second workshop. However, participants felt that the while the domains were useful and related to the key elements they had identified, the assessment scale of the model contained generic statements 
that did not have sufficient detail to enable self-assessment for Alliances, and that it did not encompass the New Zealand context, especially Te Tiriti o Waitangi. Participants felt they had the knowledge and the experience in the room to collectively construct a maturity matrix that was more suitable for the New Zealand health system.

Notes from the first workshop, agenda for the second workshop (Appendix 8) and the outline of the maturity matrix were sent to the participants two days prior to the workshop. Of the 12 participants that had accepted, 10 attended the workshop. One participant who attended the first workshop could not attend the second workshop owing to work demands. This participant offered to contribute via email. Two participants who did not attend the first workshop were able to attend the second workshop, albeit one was about an hour late.

The room set-up was similar to the first workshop with three tables to enable small group work. I had outlined the maturity matrix template for the key elements using butchers paper. I considered using laptops at each table to record the information, however, did not feel that this method would facilitate group work. The risk with this approach would have been that one person would spend their time typing the information and not being able to participate in group discussions. This method would also not provide the visual representation of the maturity matrix for the overall group, that is, it would be hard for participants to see the whole group work and amend or add to it through an iterative process. Therefore, I decided to use butcher paper with Post-it notes. I posted the butchers paper with the maturity matrix template for each element on the walls. At each table, participants had access to notes from the first workshop along with post-it notes, pens and markers.

The facilitator began the workshop at 09:30 hours with introductions as there was one new person in the room. I then presented key findings from the first workshop. This included the key elements that need to be present to increase the chances of successful implementation of LST initiatives, and the definitions and outcome descriptors for these elements. Doing so refreshed the memory of participants from the first workshop and brought the new participant to the same level of understanding. The facilitator reminded participants of their rights, how the information would be used and ground rules for the workshop. Information used here was the same as the one used in the first workshop.

The workshop comprised three sessions. My role in the workshop was a participant observer. I provided clarifications (when asked) and assisted the facilitator with data collection. 


\section{Session one - Finalising key element definitions and outcome descriptors}

This session was to recap the work of the first workshop and an opportunity for the participants to add given they have had a week to reflect.

Each table was provided with the 11 key elements, definitions and outcome descriptors from the first workshop, on an A3 in a table form (outline shown in Table 7.4). Participants were asked to discuss within their small groups and add to this information. Participants were directed to start with the elements that had the least information. The $A 3$ outline (Table 7.4) was used to record group discussions.

\begin{tabular}{|c|c|c|}
\hline Key element & Description of the element & $\begin{array}{c}\text { Outcome descriptor - what } \\
\text { does established element } \\
\text { demonstrate }\end{array}$ \\
\hline $\begin{array}{l}\text { E.g. Alliancing } \\
\text { way of working }\end{array}$ & $\begin{array}{l}\text { Alliancing is a clinically led, community engaged } \\
\text { forum that brings different component parts of } \\
\text { the health system together with the aim of } \\
\text { transforming services so that they are best for the } \\
\text { patient and best for the system. Alliancing is a way } \\
\text { of working, reliant on trusted relationships and } \\
\text { supported by enablers critical to continuous } \\
\text { improvement. Successful Alliances bring } \\
\text { alignment to multiple organisations and services } \\
\text { present in a complex system, to achieve a } \\
\text { common set of patient-centred goals. Alliancing is } \\
\text { not about managing finances or organisational or } \\
\text { professional accountabilities (Gauld, 2017). }\end{array}$ & $\begin{array}{l}\text { Trust, shared sense of purpose } \\
\text { and sustainability }\end{array}$ \\
\hline \multicolumn{3}{|l|}{ Group feedback } \\
\hline
\end{tabular}

Table 7.4: Finalising key element definitions and outcome descriptors

\subsubsection{Construction of the self-assessment maturity matrix}

Participants constructed the maturity matrix during sessions two and three. The outline of the maturity matrix and the development process was based on the study by Kirk, et al. [155], which created a framework to measure service improvement progress over time following implementation of an intervention across England and Wales (appointment of Cardiac Genetic Nurses) to enhance inherited cardiac conditions services. The maturity matrix was created using key stakeholders through a consensus approach. The matrix contained five domains with a sixpoint scale to locate progress from 'emerging' to 'established'. The matrix was used as a selfassessment tool by the Cardiac Genetic Nurses between 2009 and 2011. 
The tool had three components:

1. What does the established element demonstrate? (Outcome descriptors from the first workshop)

2. What will show this? (Indicators)

3. What should we expect to see in beginning, emerging, established, and excellence level? (Expected maturity at each stage)

Table 7.5 shows the maturity matrix outline used for this research that was reproduced with author's permission.

The facilitator, with my assistance, introduced to participants the outline of the maturity matrix, its purpose and principles that underpinned the use of the maturity matrix.

\begin{tabular}{|c|c|c|c|c|c|}
\hline \multicolumn{6}{|c|}{ Key element 1} \\
\hline \multirow{2}{*}{$\begin{array}{l}\text { Outcome } \\
\text { descriptor }\end{array}$} & \multirow{2}{*}{$\begin{array}{l}\text { Indicators } \\
\text { (what will } \\
\text { show this?) }\end{array}$} & \multicolumn{4}{|c|}{ Maturity scale } \\
\hline & & Beginning $=0$ & Emerging = 1 & Established = 2 & Excellence = 3 \\
\hline \multirow[t]{3}{*}{$\begin{array}{l}\text { What does } \\
\text { established } \\
\text { element } \\
\text { demonstrate? }\end{array}$} & $\begin{array}{l}\text { Whole-of- } \\
\text { system } \\
\text { indicator }\end{array}$ & $\begin{array}{l}\text { Nothing in } \\
\text { place }\end{array}$ & $\begin{array}{l}\text { Something in } \\
\text { place }\end{array}$ & $\begin{array}{l}\text { This is what } \\
\text { good looks like }\end{array}$ & $\begin{array}{l}\text { Outstanding e.g. } \\
\text { health and } \\
\text { social } \\
\text { integration }\end{array}$ \\
\hline & $\begin{array}{l}\text { Equity } \\
\text { indicator }\end{array}$ & $\begin{array}{l}\text { Nothing in } \\
\text { place }\end{array}$ & $\begin{array}{l}\text { Something in } \\
\text { place }\end{array}$ & $\begin{array}{l}\text { This is what } \\
\text { good looks like }\end{array}$ & $\begin{array}{l}\text { Outstanding e.g. } \\
\text { health and } \\
\text { social } \\
\text { integration }\end{array}$ \\
\hline & $\begin{array}{l}\text { Te Tiriti o } \\
\text { Waitangi } \\
\text { indicator }\end{array}$ & $\begin{array}{l}\text { Nothing in } \\
\text { place }\end{array}$ & $\begin{array}{l}\text { Something in } \\
\text { place }\end{array}$ & $\begin{array}{l}\text { This is what } \\
\text { good looks like }\end{array}$ & $\begin{array}{l}\text { Outstanding e.g. } \\
\text { health and } \\
\text { social } \\
\text { integration }\end{array}$ \\
\hline
\end{tabular}

Table 7.5: Maturity matrix outline adapted from Kirk, et al. [155]

\section{Session 2 - Developing indicators}

There were three domains identified for the indicators: whole of system approach, equity perspective, and Te Tiriti o Waitangi perspective (Table 7.5). Each group was asked to select a domain to work on. This was confirmed by going around the room to ensure that each of the three groups had one unique domain to work with.

Participants were then asked to discuss within their group on what an indicator for their domain would look like. Once the group reached agreement, the group recorded this using the post-it 
note and posted on the relevant section of the butchers paper. The groups then moved along with the facilitators signal until they finished identifying at least one indicator for their domain for all 11 elements. The groups were then asked to walk around the room to see what other groups had identified as indicators, discuss and add or amend the indicator(s) for their domain. This also gave an opportunity for all participants to see all the indicators identified.

\section{Session 3-Identifying maturity at each stage}

The groups were asked to discuss what the maturity looked like at each stage. Participant requested that they continue to work with the domain for which they developed indicators. Each group started with a different element and worked around the room. Information on what each stage looked like was recorded using post-it notes on the relevant sections of the butcher paper. The groups were moving at different pace; therefore, the facilitator's role was to ensure that groups were moving around. Once the groups finished the first round, two iterative processes were used to refine the maturity matrix. In the first iteration, participants were asked to walk around the room in their groups to see what other groups had identified and add to the information using post-it notes or a different coloured pen. The facilitator then walked around the room and reviewed the maturity matrix to ensure that each element had at least three indicators and three post-it notes describing each maturity stage, one for each indicator domain. Where a gap was identified in the maturity scale, the facilitator highlighted this by posting blank post-it notes.

In the second iteration, participants were asked to walk around the room individually to review the matrix and add to the information using post-it notes or a different coloured pen. Participants were asked to pay attention to the blank post-it notes to rectify the gaps.

As a final stage, the facilitator asked if participants wanted another iteration round to finalise the maturity matrix, but this was declined. Photo 7.1 shows the data collection process used to construct the maturity matrix. 


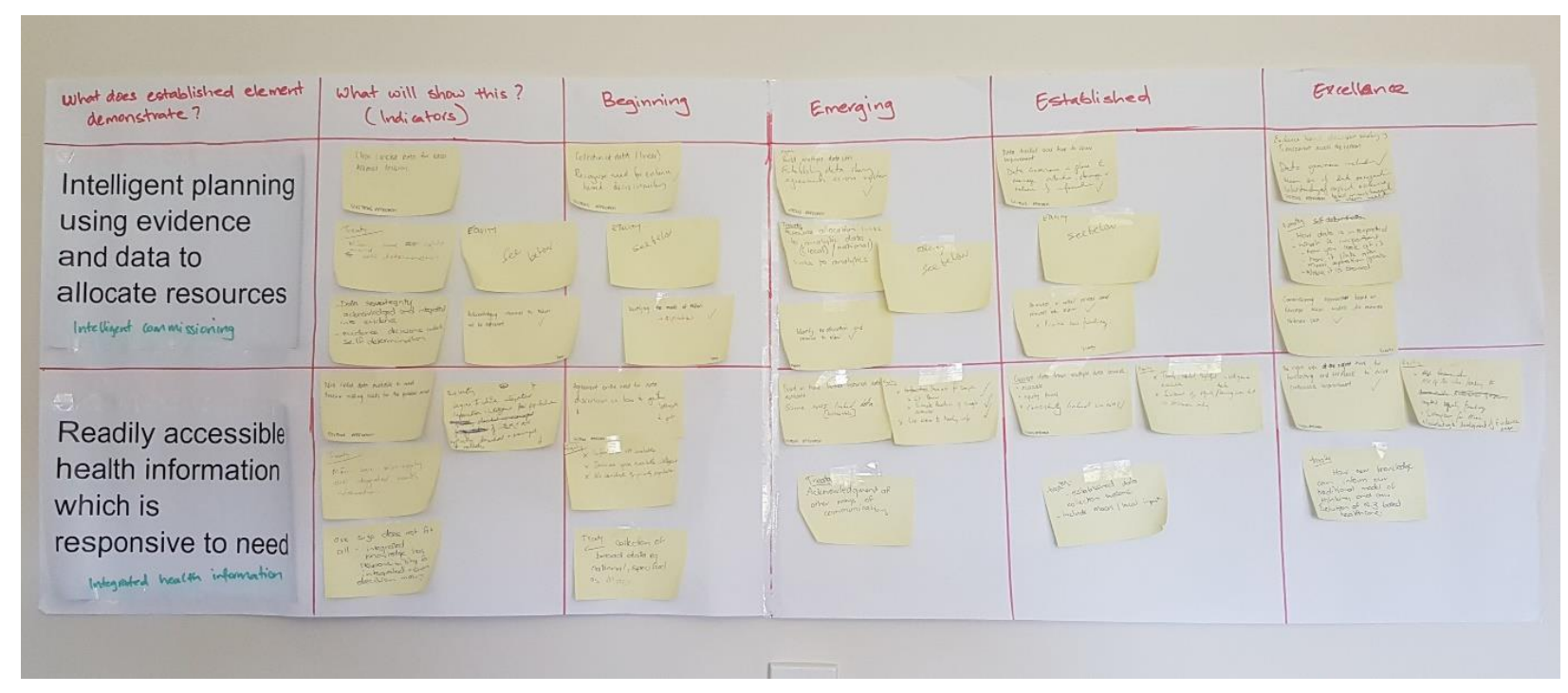

Photo 7.1: Construction of maturity matrix

\subsubsection{Concluding session}

I thanked participants for their attendance and contribution to the research and explained the next steps. Participants indicated that they would like to continue their contribution in finalising the maturity matrix via email, which was done following the workshop. The workshop finished at 15:30 hours.

\subsubsection{Data analysis}

Data from the rest of the workshop, which was focused on construction of the maturity matrix, was collected using a pre-outlined template on butcher paper. I transcribed the information, which formed the first version of the maturity matrix. This version was shared with workshop participants following the workshop for their feedback. The second version of the maturity matrix that incorporated post-workshop feedback from participants was used for testing with first Alliance leadership team (phase six).

Following both workshops, a thorough analysis was undertaken to finalise findings of the workshops. The audio recorded sessions from both workshops were transcribed by a professional transcriber. NVivo computer software programme was used to group key thematic groups of texts from the transcripts. I coded deductively, based on the codes generated for key elements, contexts, mechanisms and outcomes in phase three (interviews) and created new codes for additional elements and CMOs identified by workshop participants. Direct quotes from the transcripts were also used with all identifying information removed to protect participants' anonymity. 


\subsection{Phase five: Online survey}

The aim of the survey was to consolidate the key elements and further test my initial propositions and perspectives gained from interviews and workshops with those involved in the implementation of the SLM programme in DHBs and PHOs. These participants had an inside knowledge and experience of what influenced the successful implementation of the SLM programme in their districts.

An online survey is a self-administered questionnaire that uses the web as the data collection mode [187]. It is a quick, efficient and inexpensive way of collecting data from a targeted sample that is large and dispersed. It is especially suitable for a questionnaire that involves a skip pattern and contains open ended questions [187]. The electronic data collection makes transcription unnecessary and participants are more likely to provide more comprehensive answers to questions than on paper surveys [187].

The survey design followed realist guidance in terms of sampling, participant selection and question design [49]. The survey did not aspire to statistical validation or 'the final truth'. Instead it sought a closer understanding of the programme architecture to successfully implement LST initiatives consolidated from prior research phases. The online survey was anonymous by design to encourage participants to provide honest answers about their experience on the key elements that supported successful implementation of LST initiatives, such as the SLM programme, and contexts and mechanisms that influenced these initiatives.

\subsubsection{Development of the survey instrument}

The survey instrument was designed and conducted using the guide published by Manzano [49] and was developed based on findings from earlier research phases. It included 10 key elements that support successful implementation of LST initiatives with a short definition and refined initial propositions that influenced these initiatives. Participants were asked their level of agreement on key elements and refined propositions using a five-point Likert scale (strongly disagree, disagree, neither disagree nor agree, agree, and strongly agree). Using open-ended questions, participants were then asked to identify further contexts and mechanisms that influenced the implementation of the SLM programme in their district. Variables used were categorical, such as Likert scales, and qualitative, such as open-ended questions for contexts, mechanisms and outcomes. Qualtrics survey software was used to collect survey data. 


\subsubsection{Testing the survey}

The questionnaire mirrored the question structure used for the interviews; however, it included the information gained from earlier research phases (phases one, two, three and four).

The test version of the survey contained eight questions:

- The first two questions asked for demographic data on whether participants worked in a $\mathrm{DHB}, \mathrm{PHO}$ or other organisation and whether their main role at the organisation was a health care professional, management or leadership, consumer advocate or other. 'Other' included roles such as a quality improvement lead or analyst.

- Question three listed 10 key elements and definitions, refined from literature review, interviews and the workshops, and asked participants' level of agreement on each of the elements using a five-point Likert scale (strongly disagree, disagree, neither disagree nor agree, agree, and strongly agree).

- Question four described eight initial propositions, refined from interviews and workshops, that influenced implementation of LST initiatives. Participants were asked their level of agreement on key elements and refined initial propositions using a five-point Likert scale (strongly disagree, disagree, neither disagree nor agree, agree, and strongly agree).

- Questions six, seven and eight asked participants, using open-ended questions, for additional contexts, mechanisms and effects (CMOs) that influenced the successful implementation of the SLM programme in their district.

- The last open-ended question sought final comments from participants.

I tested the survey with three participants from the sector, the SLM programme clinical lead and my supervisors. Changes to the questionnaire were made based on the feedback that included simplifying definitions for key elements and refined initial propositions to make it clear and concise, and reducing the estimated time to complete the survey from 20 to 10 minutes. The testing process also assessed if the survey platform was working for distribution of the survey and maintaining anonymity of responses.

\subsubsection{Final survey instrument}

The final version of the survey contained nine questions, with one additional question added to improve logic flow of the survey. It was added following question four on refined initial propositions that asked participants if they were aware of other factors that influenced the implementation of the SLM programme in their districts with the binary choice of 'yes' and 'no'. 
If the participant chose 'yes' the survey progressed to the next question that asked them to list the factors. If the participant chose 'no', the survey skipped three questions on additional CMOs and proceeded to the final question.

\subsubsection{Selection of participants}

A re-useable anonymous survey link was emailed (Appendix 9) to 65 potential participants that included DHB leads for the SLM programme and PHO Chief Executives. The email included the research information sheet (Appendix 3). First receivers of the survey were invited to participate in the study by completing the survey and were asked to forward the email with the survey link to other people in their district and organisation that were or are involved in the implementation of the SLM programme. This enabled snowball sampling to identify further participants.

A reminder was sent two weeks following the first invite. The final reminder was sent three weeks following the first reminder.

Fifty-one participants completed the survey: 22 from DHBs, 28 from PHOs and one that did not identify them-self as a DHB or PHO employee.

\subsubsection{Data analysis}

Qualtrics survey software was used to analyse the survey data. Categorical survey data was also exported to Microsoft Excel for further analysis to create tables and charts.

NVivo computer software programme was used to group key thematic groups of qualitative data from the free text comment fields. I coded responses deductively based on the codes generated for key elements, contexts, mechanisms and outcomes in phase three (interviews) and created new codes for additional CMOs identified by survey participants. Direct quotes from the survey responses were also used with care taken to ensure that the text did not contain any identification information.

\subsection{Phase six: Field testing of the maturity matrix}

The aim of phase six was to test the maturity matrix with three Alliance leadership teams (ALTs) to determine if: it was functioning as a learning tool; it helped Alliances see where they were on the improvement journey; it helped Alliances identify areas of improvement; it acted as a resource to create a stimulus for change; and if it was easy and practical to use. Feedback was also sought from the Māori Health Strategy and Policy team at the MoH to ensure that Te Tiriti principles were accurately reflected in the matrix. The maturity matrix was refined following each stage of the testing. 
Following the principles of realist research to seek diverse views to validate, falsify, refine and modify the maturity matrix [49], ALTs were chosen based on the size of the population the DHB was serving and my prior knowledge of the ALT. The prior knowledge was based on my experience working as the SLM programme lead and included factors such as the membership of the ALT, how long they have existed, level of personnel changes in the ALT, quality of the SLM plan, and improvement culture and capability.

I approached the ALT chair personally or via email, and subsequently emailed the research information sheet (Appendix 3) and the consent form (Appendix 10). The ALT chairs discussed the invitation to participate in the study with their teams and confirmed their decision to participate. Once participation, date and time of the testing was confirmed by ALT chairs, further information was sent that included a coversheet memo to describe the purpose, background and testing process. Participants were asked to sign and return their consent forms either by email to me or hand it to me in person on the day of the testing. I also requested copies of the ALT membership lists so I could keep track of who had returned their consent form. On the day of the testing, with the help of the secretariat, I collected consent forms and reconciled with the membership list to ensure that all participants in the room had provided written consent to participate in the testing process.

The testing process was facilitated by the SLM programme clinical lead for two ALTs. The SLM programme clinical lead was unavailable for one of the ALT meetings, so for this meeting, one of the participants who was involved in the development of the maturity matrix and was a member of the ALT offered to facilitate the testing process. Both facilitators signed a confidentiality agreement (Appendix 11).

The regular Alliance meetings are usually set at the beginning of the year to allow sufficient notice for members. Given the seniority and the wide-ranging membership of ALTs, arranging a meeting at short notice was logistically difficult. Therefore, ALTs' regular meetings were used for the testing process. One hour was requested for the testing process either during the usual agenda, or before or after the ALT meeting. I used the Victoria University Faculty Grant to pay for my travel costs to attend ALT meetings outside of Wellington.

Of the first three ALTs that were invited to participate in the testing process, all of them accepted. The ALTs saw the testing process as an opportunity to reflect on their journey as an Alliance and identify areas for development. 


\subsubsection{Alliance leadership team one}

ALT one was a small DHB with a population of fewer than 200,000 [188]. The district had one PHO providing primary care services. The ALT membership included representatives from the DHB, hospital, PHO, pharmacy, Māori health providers, Iwi, consumer, general practice and the Accident Compensation Corporation. The total number of participants for the testing were 16 plus facilitator and one secretariat.

One of the ALT members who participated in the workshops to develop the maturity matrix was very keen for their ALT to use the tool to reflect where they were as an ALT. The SLM programme clinical lead who was supposed to facilitate the testing process was unavailable on the day of the ALT meeting. The ALT member agreed to facilitate. The meeting was scheduled for 14:30 to 17:30 hours on 24 October 2019 at the PHO office. I was invited to attend the meeting from 14:30 hours with about an hour on the agenda for the testing process.

The testing began with my presentation on the overview of the research including research questions and the method used to develop the maturity matrix, the outline of the maturity matrix, the principles underpinning the maturity matrix, and the purpose of testing. I confirmed my attendance in my capacity as a researcher, participants' rights, research confidentiality, and the ground rules for the testing process. Once I finished my presentation, the facilitator then asked participants to break into small groups of three-to-four and assigned each group two elements to score against and provide feedback on the maturity matrix. Each group provided feedback to the wider group on the description of elements, the maturity scale content, and their score for the element. The group feedback session was audio recorded and transcribed later by a professional transcriber. As a participant observer, I provided clarifications on some of the terminology used and took notes from the group feedback session.

The testing process took about 60 minutes excluding the presentation. At the end of the session, the facilitator asked three concluding questions (Box 7.1).

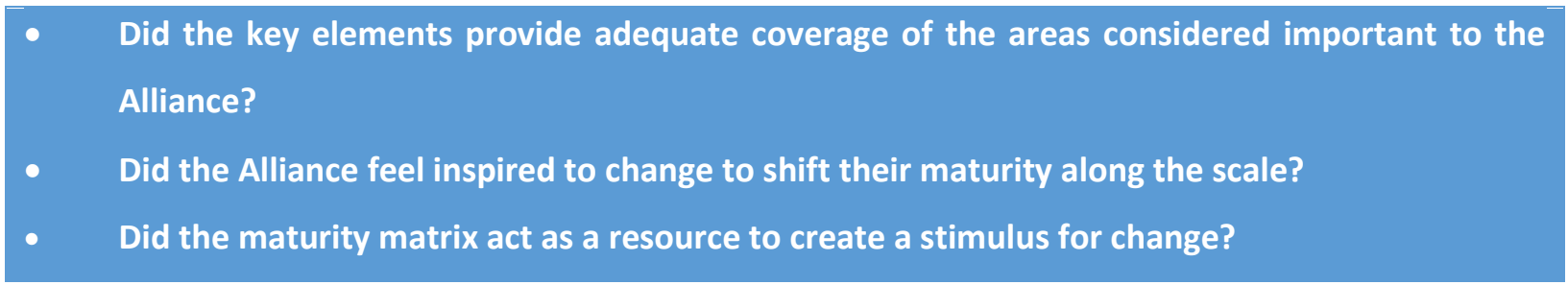

Box 7.1: Three concluding questions for the ALT 
I used the audio-recording and my notebook to record answers to these questions. Participants were welcomed to hand in any written notes and an opportunity to provide further feedback following the meeting via email. No further feedback was received.

\subsubsection{Alliance leadership team two}

ALT two was a mid-sized DHB with a population of 300,000 to 350,000 [188]. The district had one PHO providing primary care services. However, the DHB served the population living in a large geographical spread with large pockets of older population living in rural. Given its geographical diversity, it is often challenging to have all the members of the ALT meet face-to-face. Therefore, the ALT had learnt to be comfortable with working remotely using technology such as zoom meeting. The ALT had undergone significant changes in the last 18 months and were still in a formation stage. The ALT had broad membership from across the primary, hospital and community services and had an independent chair.

The testing was undertaken on 15 November 2019 at the DHB premises. Owing to the meeting having a full agenda, the chair proposed for the testing of the maturity matrix to follow the ALT meeting. The ALT members had the option to stay back and participate in the testing process. On the day prior to the meeting, the ALT meeting was cancelled owing to a significant event in the district that required attendance of some of the ALT members. The chair contacted me about this and discussed the option of either rescheduling the testing process or continuing with a smaller group. On recommendation of the chair, the testing process continued as scheduled.

The ALT had a membership of 16 , seven of whom participated in the testing process. The meeting started with six participants with another joining in about 20 minutes into the process. Participants were mostly of clinical background. Two participants, along with the facilitator joined in using zoom meeting technology. Written consent forms were collected from all participants at the beginning of the meeting. Participants on the zoom meeting emailed their consent forms to me. The meeting began with introductions followed by the facilitator introducing the outline of the maturity matrix, how it was developed, its purpose, purpose of the testing process, researcher's conflict of interest and participants' rights including research confidentiality. The testing process was paused when the seventh participant joined in. This was to allow the facilitator an opportunity to introduce the participant to the people and the process.

The participants scored themselves against the key elements. The ALT was able to score on the first six elements in the hour they had. A group discussion then followed on the maturity matrix. The facilitator asked three questions (Box 7.1). 
I used the audio-recording and my notebook to record answers to these questions. The meeting was concluded at 15:35 hours. Participants were encouraged to hand in any written notes and an opportunity to provide further feedback following the meeting via email. No further feedback was received.

\subsubsection{Alliance leadership team three}

ALT three was a mid-sized metropolitan DHB with a population of 300,000 to 350,000 [188]. Multiple PHOs provided primary care services in the district. The chair of the ALT was keen to use the testing process for the ALT's end of the year reflection process.

The meeting was scheduled for 16:30 to 18:00 hours on the 12 December 2019 at the DHB premises. With the time of the year and this being the last meeting of the ALT, attendance at the meeting was lower than usual. The ALT had 27 members, 14 of whom participated in the testing process. Attendees were a mix of clinical and operational leaders from across the primary, secondary and public health sectors. The facilitator joined via zoom meeting.

The testing began at 17:10 hours with my recap on the overview of the research including research questions and the method used to develop the maturity matrix, the outline of the maturity matrix, the principles underpinning the maturity matrix, and the purpose of testing. I confirmed my attendance in my capacity as a researcher, participants' rights', research confidentiality, and the ground rules for the testing process.

When I finished my presentation, the facilitator asked participants to break into small groups of two-to-three and assigned each group two elements to score against and provide feedback on the maturity matrix. Each group provided feedback to the wider group on the maturity matrix and their score for the element. The group feedback session was audio recorded and transcribed later by a professional transcriber.

The testing process took about 45 minutes excluding the presentation. At the end of the session, the facilitator asked three questions (Box 7.1).

I used the audio-recording and my notebook to record the answers to these questions. The meeting was concluded at 18:15 hours. Participants were encouraged to hand in any written notes and an opportunity to provide further feedback following the meeting via email. No further feedback was received. 


\subsubsection{Māori Health Strategy and Policy team at the $\mathrm{MoH}$}

Following testing with the three ALTs, I shared the maturity matrix with the manager and the Principal Advisor (Equity) of the Māori Health Strategy and Policy team at the MoH to seek their feedback on the matrix. I asked the manager and the Principal Advisor to review the maturity matrix to ensure that Te Tiriti principles were accurately reflected across the maturity scale for all the key elements, with particular attention to two elements: commitment to Te Tiriti o Waitangi; and understanding of equity. The Principal Advisor provided their feedback via email, following which I met with the manager and the Principal Advisor to discuss their feedback in person. After incorporating their feedback, I shared the revised maturity matrix with them to check that I had interpreted and addressed their feedback correctly. They confirmed its accuracy.

\subsubsection{Data analysis}

Printed copies of the maturity matrix were provided to ALTS to use for the testing process. The ALTs scored themselves using the maturity matrix. Since the purpose of the testing was to determine if the maturity matrix was easy and practical for Alliances to use to gauge their maturity on the capacity and capability for implementing LST initiatives, the Alliances' scores were not collected, recorded or analysed. The scores were left with the ALTs to use as they saw appropriate.

Group discussions were used to collate feedback from participants on the content of the maturity matrix and answers to three questions relating to the areas covered in the maturity matrix, the Alliances' inspiration to change and if the maturity matrix acted as a resource to create a stimulus for change. These discussions were audio recorded and transcribed later by a professional transcriber. I recorded my observations and key points of feedback from participants in a notebook. Transcripts of group discussions and field notes were used to refine the maturity matrix following each testing process. Feedback from the Māori Health Strategy and Policy team at the $\mathrm{MoH}$ was provided via email with comments in the electronic copy of the matrix. I took notes from the meeting with the manager and the Principal Advisor. A new version of the maturity matrix was created incorporating participants' feedback at each stage of the testing. 


\subsection{ETHICAL CONSIDERATIONS}

Ethical approval for the research was requested from two committees: the national Health and Disability Ethics Committee (HDEC) and the Victoria University Human Ethics Committee (HEC).

A letter from HDEC dated 19 March 2019 confirmed that this research was outside the scope of HDEC. This was because the research did not involve any participants recruited in their capacity as consumers (or their relatives) of health or disability services, or as volunteers in clinical trials, or the use of health information or tissue.

Ethics approval from the HEC was sought on 19 March 2019 and was considered by the committee during April and May. The following components were given specific ethical consideration.

\subsection{Researcher's conflict of interest}

The committee's main concern related to privileges I had from my position in respect to the research population (the 'insider' perspective) being a $\mathrm{MoH}$ employee leading the SLM programme, resulting in opportunities which might not be available to other doctoral researchers, and the genuine ethical issues that might arise, such as an impact on voluntary participation. Participants in the research were senior leaders in the health sector and not a vulnerable group that was put at risk from declining to participate. However, the committee highlighted that most doctoral researchers are not able to ask health system employees to be funded to attend full day workshops, or able to access meetings of Alliances for testing of the maturity matrix. The value that organisations saw in having their employees participate had to be considered in light of my role at the $\mathrm{MoH}$.

In realist research, prior knowledge of the research subject matter and purposeful selection of participants that are 'information rich' are essential tenets $[4,49]$. This allows the researcher to use personal experience to identify and test theories from within the system rather than 'looking in'. Therefore, my inside knowledge of the health system, and three years of first-hand experience of observing the key elements that support implementation of the SLM programme and how contexts influenced the successful implementation of the programme was invaluable to the research. It helped me to invite the right participants for the study, both in terms of seniority and experience in the sector.

The workshops and Alliance meetings were facilitated by the SLM programme clinical lead who was a part-time $\mathrm{MoH}$ and a $\mathrm{PHO}$ employee. This added another element of conflict. I had used 
the SLM programme clinical lead as a facilitator as they had led the co-development of the SLM programme and therefore had the relevant skills, subject matter expertise and credibility in the health sector. The facilitator's mana with the sector had proven to be a strength in the past for facilitating workshops attended by senior leaders and clinicians.

Prior knowledge about the functionality and makeup of ALTs were valuable in the selection process for the field testing of the maturity matrix. I was able to invite three teams that differed in their membership, size of the district they served, and the state of their local relationships.

However, both the facilitator and I brought (unconscious) bias to the research with our prior knowledge and experience. We had pre-conceived notions of what worked (and did not work) with the implementation of the SLM programme, our ideas about the programme architecture to successfully implement LST initiatives, and our opinions about participants that might have caused us to weigh their contributions accordingly. We were not observers from the outside asking questions; our questions could steer participants towards certain responses. I managed this bias by selecting a wide range of data collection and analysis strategies that enabled me to actively search for rival theories and build rigour to my initial propositions. I also selected participants (for interviews and workshops) who were senior and experienced, and who would not hesitate to share their opinions, or challenge or disagree with others' opinions.

Our dual roles created both perceived and real conflicts of interest as highlighted by the HEC. As such, a number of precautions were taken to manage the risks that could potentially arise from the conflict. I worked with HEC and my supervisors to ensure that participants were provided with clear written information in the participant information sheet that participation in the research was voluntary, the conflict of interest arising from my dual role was clearly declared, and how the information sought from them was going to be used. Participant information sheet was shared as part of the process used to obtain written consent and at the beginning of interviews, workshops and ALT meetings. Workshops were held using university rooms, which reinforced the purpose of the workshop and separation from the $\mathrm{MoH}$ work. Interviews were held either at the participants' place of work, at the university or in public meeting places such as a café. The ALT meetings were held at participants' usual DHB or PHO premises.

The research was approved by HEC on 12 June 2019 (reference number 27356). Subsequently, I sought two amendments to the approval from HEC, which were granted in July and August 2019 respectively. 
The first request in July sought an amendment to include the use of digital technology such as zoom meeting as an interview method. The participant information sheet was amended to include this additional method for interview participants. I also sought an exemption for participants who were company partners, directors, or independent contractors or clinicians from seeking Chief Executive permission where they deemed it impractical. This only applied to participants that held senior level positions and had delegations that were equivalent to that of a Chief Executive (e.g. partners and directors of private companies, or independent clinicians or contractors).

The second request for amendment in August sought an exemption to obtain Chief Executives' permission for the online survey data collection. The online survey was anonymous and would take about 10 minutes to complete. Given the anonymity and the length of the survey, I did not anticipate the completion of the survey to be a burden or a reputational risk to the organisations.

\subsection{Informed consent}

The participant information sheet and the consent form were sent to all participants invited to participate in workshops, interviews and field testing of the maturity matrix. The participant information sheet outlined the aim of the research, my dual roles and conflict of interest, ethics approval reference number, and what was required from participants. It also explained how the information was going to be used, the product of the research, participants' rights, and my contact details as well as those of HEC convener and my supervisor for any further questions. The consent form sought written confirmation from participants that they understood the information sheet, and agreed to participate in audio-recorded interview, workshop or ALT meeting. The consent form also outlined research confidentiality requirements, participants' right to withdraw from the research, how the information provided by them will be used, when information will be destroyed, and if they were interested in receiving the final research thesis and publications. Where relevant for workshops and interviews, the participant information sheet was sent to Chief Executives of the participants' employing organisation and written consent sought to permit participants to participate in the research. This was especially important for workshops where the attendance was funded by the participants' employing organisation.

With the exception of those participating in the online survey, all participants provided written consent prior to participation in the research. 


\subsection{Research confidentiality}

Research confidentiality was important to protect anonymity of participants and their employing organisations. It also provided a safe environment for participants to be free and frank in sharing their experiences. All participants were given written assurances through the information sheet and consent form that their information will be stored securely and shared only with research supervisors, the facilitator and the transcriber as necessary. Participants, through the consent form, agreed that they may share their contributions with others but not the contribution of other participants.

The facilitator signed a confidentiality agreement (Appendix 11) to only discuss information shared by participants with the researcher, the research supervisors and potentially with participants.

The transcriber signed a confidentiality agreement (Appendix 12) to store all information relating to the research securely, to not discuss information with anyone except the researcher and to destroy all files upon completion of transcriptions.

Given the small size of New Zealand health sector and the well-known profiles of senior leaders and clinicians, I was careful in my description of participants and their contributions in order to preserve their anonymity. Consequently, this limited my descriptions of participants in this chapter, including description of ALTs. Where I used direct quotes from participants, I removed all information that could potentially identify the participant or their employing organisation.

All research information, including recordings and transcripts, was stored on secure devices with password access only and will be destroyed on 31 October 2022 or once the doctoral research requirements are fulfilled (whichever comes first). The interview transcripts were coded to protect anonymity of interviewees. Transcripts of group discussions separated speakers with a code 'NS' (new speaker) and did not identify speakers. 


\subsection{Summary of Part B}

Part $\mathrm{B}$ described the theoretical underpinnings and the rationale for using a realist approach. The research took into consideration the five key ideas in realism [47]. First, the research recognised that structures, and institutional, social and political conditions in health systems are real and have real effects on LST initiatives, such as the SLM programme. The research used insights and knowledge gained from the implementation of the SLM programme to glean initial propositions to inform the programme architecture for LST initiatives in the New Zealand health system.

Second, that experiences of agents in the system shape their reasoning and behaviours. Participants in the research were a broad range of stakeholders, most of whom were involved in the SLM programme implementation or those charged with making major strategic decisions about where effort goes towards supporting implementation of LST initiatives. The research also included participants that were considered leaders in complexity thinking, both in New Zealand and overseas, including three participants who were senior leaders from banking, construction and organisational transformational sectors. These participants were 'information rich' to inform my research questions.

Third, that understanding system dynamics require a systems mind set. While it is difficult to know the final truth about how and why implementation of LST initiatives are successful (or not), a realist logic of enquiry, nested within the framing of complex adaptive systems, could lead to a closer understanding of the programme architecture for successful implementation of these initiatives.

Fourth, the organisational, social and political contexts influence the programme architecture. Lastly, these conditions trigger underlying, unseen mechanisms that shape the reasoning and behaviours of health system leaders and those involved in the implementation of LST initiatives such as the SLM programme and influence the successful implementation of these initiatives.

The research phases one (theory gleaning), two (literature review), three (interviews), four (workshop one) and five (online survey) analysed and described the programme architecture for LST initiatives. The maturity matrix constructed in phase four (workshop two) and tested in phase six with Alliance leadership teams supports conscious and deliberate learning for health system leaders to increase their understanding of this programme architecture. 
Table 8.1 shows the map of research questions, theory of realist ideas, and methods for data collection and analysis for the six research phases. The next part of the thesis reports the findings of the research. 


\begin{tabular}{|c|c|c|c|c|c|}
\hline $\begin{array}{l}\text { Research } \\
\text { phase }\end{array}$ & Research question & Application of key realist ideas & Description of realist ideas & Data collection & $\begin{array}{l}\text { Data analysis } \\
\text { technique }\end{array}$ \\
\hline $\begin{array}{l}\text { Phase } 1 \text { - } \\
\text { Theory } \\
\text { gleaning }\end{array}$ & $\begin{array}{l}\text { RQ1 - Key } \\
\text { elements } \\
\text { RQ3 - Contexts and } \\
\text { mechanisms }\end{array}$ & $\begin{array}{l}\text { Using complex adaptive system ideas to } \\
\text { develop propositions to test }\end{array}$ & $\begin{array}{l}\text { Use of the SLM programme to identify capacity and } \\
\text { capability needed to support successful } \\
\text { implementation of an LST initiative and uncover how } \\
\text { social, cultural and political conditions influence its } \\
\text { implementation }\end{array}$ & $\begin{array}{l}\text { My insights from the } \\
\text { SLM programme }\end{array}$ & $\begin{array}{l}\text { Documenting initial } \\
\text { propositions }\end{array}$ \\
\hline $\begin{array}{l}\text { Phase } 2 \text { - } \\
\text { Literature } \\
\text { review }\end{array}$ & $\begin{array}{l}\text { RQ1 - Key } \\
\text { elements } \\
\text { RQ2 - Maturity } \\
\text { matrix } \\
\text { RQ3 - Contexts and } \\
\text { mechanisms }\end{array}$ & $\begin{array}{l}\text { Evidence from literature about } \\
\text { successful implementation of LST } \\
\text { initiatives }\end{array}$ & $\begin{array}{l}\text { Definition of LST initiative that encompass both the } \\
\text { complexity of interventions and the complexity of } \\
\text { context in which these are implemented } \\
\text { Evidence in literature of key elements and contextual } \\
\text { factors that influence implementation of LST } \\
\text { initiatives } \\
\text { Evidence in literature of learning tools used to assess } \\
\text { improvement progress }\end{array}$ & $\begin{array}{l}\text { Search of published } \\
\text { and grey literature }\end{array}$ & Document analysis \\
\hline $\begin{array}{l}\text { Phase } 3 \text { - } \\
\text { Interviews }\end{array}$ & $\begin{array}{l}\text { RQ1 - Key } \\
\text { elements } \\
\text { RQ3 - Contexts and } \\
\text { mechanisms }\end{array}$ & $\begin{array}{l}\text { Selection of a wide range of participants } \\
\text { who were considered leaders in } \\
\text { complexity thinking from New Zealand } \\
\text { and overseas } \\
\text { Teacher-learner cycle in interviews } \\
\text { Using complex adaptive system ideas to } \\
\text { construct mid-range theories }\end{array}$ & $\begin{array}{l}\text { Testing key elements and contextual factors with } \\
\text { senior leaders who are contracted as experts to } \\
\text { advise on or evaluate the implementation of LST } \\
\text { initiatives (New Zealand and overseas) } \\
\text { Exploring counterfactual ideas - looking for reasoning } \\
\text { and behaviours they came across as experts of the } \\
\text { system } \\
\text { Testing key elements and contextual factors with } \\
\text { senior leaders from non-health sector to compare } \\
\text { similarities and differences }\end{array}$ & $\begin{array}{l}\text { Audio recordings } \\
\text { Transcripts }\end{array}$ & $\begin{array}{l}\text { Codes in NVivo } \\
\text { software } \\
\text { Retroduction to } \\
\text { identify realist } \\
\text { mechanisms }\end{array}$ \\
\hline $\begin{array}{l}\text { Phase } 4 \text { - } \\
\text { Workshops }\end{array}$ & $\begin{array}{l}\text { RQ1 - Key } \\
\text { elements } \\
\text { RQ2 - Maturity } \\
\text { matrix } \\
\text { RQ3 - Contexts and } \\
\text { mechanisms }\end{array}$ & $\begin{array}{l}\text { Selection of a wide range of participants } \\
\text { who had experience of leading or } \\
\text { implementing the SLM programme } \\
\text { Using complex adaptive system ideas to } \\
\text { construct mid-range theories } \\
\text { Using complex adaptive system ideas to } \\
\text { construct a tool to support a learning } \\
\text { system }\end{array}$ & $\begin{array}{l}\text { Testing key elements and contextual factors with } \\
\text { senior leaders who are responsible for leading } \\
\text { implementation of LST initiatives in New Zealand } \\
\text { health system } \\
\text { Exploring counterfactual ideas - looking for reasoning } \\
\text { and behaviours shaped by their individual } \\
\text { experiences } \\
\text { Using experience of system leaders to determine } \\
\text { what maturity for key elements looks like at different } \\
\text { stages }\end{array}$ & $\begin{array}{l}\text { Audio recordings } \\
\text { Transcripts } \\
\text { Pre-outlined } \\
\text { butchers paper } \\
\text { Pre-outlined A3 data } \\
\text { collection templates } \\
\text { Field notes }\end{array}$ & $\begin{array}{l}\text { Real-time analysis } \\
\text { Codes in NVivo } \\
\text { software } \\
\text { Retroduction to } \\
\text { identify realist } \\
\text { mechanisms } \\
\text { Document analysis } \\
\text { Inductive for creation } \\
\text { of maturity matrix }\end{array}$ \\
\hline
\end{tabular}




\begin{tabular}{|c|c|c|c|c|c|}
\hline $\begin{array}{l}\text { Research } \\
\text { phase }\end{array}$ & Research question & Application of key realist ideas & Description of realist ideas & Data collection & $\begin{array}{l}\text { Data analysis } \\
\text { technique }\end{array}$ \\
\hline $\begin{array}{l}\text { Phase } 5 \text { - } \\
\text { Online } \\
\text { survey }\end{array}$ & $\begin{array}{l}\text { RQ1 - Key } \\
\text { elements } \\
\text { RQ3 - Contexts and } \\
\text { mechanisms }\end{array}$ & $\begin{array}{l}\text { Selection of participants who had } \\
\text { experience of implementing the SLM } \\
\text { programme } \\
\text { Teacher-learner approach to question } \\
\text { design } \\
\text { Using complex adaptive system ideas to } \\
\text { construct mid-range theories }\end{array}$ & $\begin{array}{l}\text { Testing key elements and contextual factors with } \\
\text { frontline staff and middle managers who are } \\
\text { responsible for implementing the SLM programme } \\
\text { Exploring counterfactual ideas - looking for reasoning } \\
\text { and behaviours shaped by individual experiences that } \\
\text { influenced implementation of the SLM programme }\end{array}$ & $\begin{array}{l}\text { Qualtrics survey } \\
\text { software }\end{array}$ & $\begin{array}{l}\text { Qualtrics survey } \\
\text { analysis } \\
\text { Codes in NVivo } \\
\text { software }\end{array}$ \\
\hline $\begin{array}{l}\text { Phase } 6 \text { - } \\
\text { Testing } \\
\text { with ALTs }\end{array}$ & $\begin{array}{l}\text { RQ2 - Maturity } \\
\text { matrix }\end{array}$ & $\begin{array}{l}\text { Testing the programme architecture for } \\
\text { LST initiatives with a range of ALTs, who } \\
\text { had experience of implementing the } \\
\text { SLM programme }\end{array}$ & $\begin{array}{l}\text { Testing the maturity matrix to see if it acts as a } \\
\text { stimulus for change, the type of conversation it } \\
\text { stimulates, and if it can used as a resource to support } \\
\text { continuous learning }\end{array}$ & $\begin{array}{l}\text { Audio recordings } \\
\text { Transcripts } \\
\text { Field notes }\end{array}$ & $\begin{array}{l}\text { Deductive for testing } \\
\text { of the maturity matrix }\end{array}$ \\
\hline
\end{tabular}

Table 8.1: Map of research questions, theory and methods of data collection and analysis 
PART C: FINDINGS 
LST initiatives are "interventions aimed at co-ordinated, system wide change affecting multiple organisations and care providers, with the goal of significant improvements in the efficiency of health care delivery, the quality of patient care, and population-level patient outcomes" ( $p$ 422) [1]. The SLM programme is an example of a LST initiative designed to achieve system transformation in the New Zealand health system. The programme was introduced to enhance a collaborative way of working beyond organisational and professional boundaries, address health inequities and encourage continuous learning and quality improvement and was implemented through Alliances [15]. Alliances are informal networks in the New Zealand health system that were introduced to integrate the planning and delivery of health care between DHBs and PHOs [42]. The key purpose of the SLM programme is to achieve the Triple Aim outcome: improved quality, safety and experience of care; improved health and equity for all populations; and best value for public health system resource [15].

My insights and experience from leading the implementation of the SLM programme and the evaluation of the programme [121] revealed some of the programme architecture for successfully implementing the SLM programme. These insights and academic literature led to the three research questions that guided the research to analyse and describe the programme architecture that underpins efforts to successfully implement LST initiatives in the New Zealand health system.

Research question one: What are the key elements that support successful implementation of LST initiatives in the New Zealand health system?

Research question two: How do New Zealand Alliances consciously and deliberately increase their understanding of the key elements to support the notion of a learning system?

Research question three: What contexts and mechanisms influence the New Zealand Alliances' ability to successfully implement LST initiatives?

Part $\mathrm{C}$ presents the findings of the research and contains three chapters corresponding to the research questions. 


\section{Chapter 9: Key elements that support successful implementation of large-system}

transformation initiatives

This chapter answers research question one and presents the testing and refining process from an initial to the final list of key elements that need to be present to increase the chances of successful implementation of LST initiatives in the New Zealand health system.

\section{Chapter 10: The self-assessment maturity matrix}

This chapter answers research question two and presents the maturity matrix that was developed with the senior clinical and operational leaders working in the New Zealand health system at the two workshops. The chapter outlines how the maturity matrix was tested and refined and presents what was found when successive versions were then tested with the three Alliance leadership teams. The chapter also includes feedback on the maturity matrix from the MoH Māori Health Strategy and Policy team.

Chapter 11: Contexts and mechanisms that influence successful implementation of largesystem transformation initiatives

Chapter 11 answers research question three and reports on mechanisms triggered by the local and national contexts that influence a district's ability to successfully implement LST initiatives.

The three chapters together analyse and describe the programme architecture that underpins efforts to successfully implement LST initiatives in a New Zealand context. 


\subsection{KEY ELEMENTS TO SUPPORT SUCCESSFUL IMPLEMENTATION OF LST INITIATIVES}

The key elements that support successful implementation of LST initiatives were determined using the realist research design outlined in

Figure 7.1. Five methods were used: initial propositions based on my insights from the SLM programme; a literature review; workshops; interviews; and an online survey (as shown in Figure 9.1). This chapter presents the testing and refining process from an initial to the final list of key elements that need to be present to increase the chances of successful implementation of LST initiatives in the New Zealand health system.
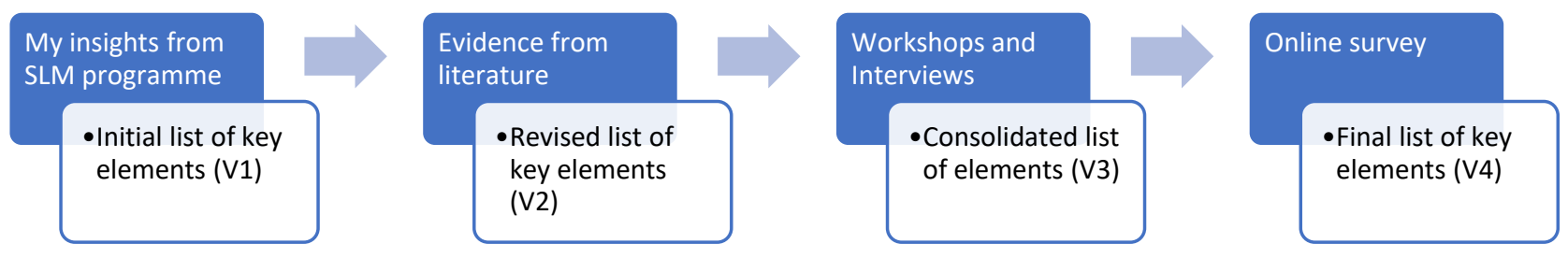

Figure 9.1: Process to finalise key elements

\subsection{Insights from the implementation of the SLM programme}

These insights evolved from my knowledge and experience of leading the implementation of the SLM programme.

Implementation of the SLM programme highlighted the variability in the way Alliances worked to develop a high-quality integrated plan and whether they were able to fully implement it in their districts. According to the SLM programme implementation guidance, a high-quality integrated plan was developed through clinically led processes involving multiple stakeholders, using evidence from health and social data, and had improvement actions that would change health outcomes [40]. This planning process, along with the full implementation of each plan and the ability to undertake reflective learning on processes and progress, indicated successful implementation of the programme.

There were several elements and contexts that I felt were causing variability in the successful implementation of the programme: an alliancing way of working; clinical leadership and engagement; the degree to which consumers were involved in the development and implementation of the SLM plans; information technology infrastructure; capacity and capability 
for quality improvement; and the use of commissioning cycles. These elements, and how they were influenced by contexts are described below.

Since, 2013, the MoH mandated DHBs to form Alliances with PHOs delivering primary care services in their districts. Most of the Alliances existed to meet this contractual requirement but did not have terms of reference that outlined the process, purpose, role clarity or 'ways of working', or did not appear to believe in the philosophy of an alliancing way of working, or both. The contractual arrangement also hindered some DHBs in bringing other local partners such as pharmacy and maternity into the Alliance because a strict interpretation of the contractual mandate narrowed the thinking of DHB and PHO system leaders.

From my observations (through the plan approval process), implementation of the SLM programme revealed the functionality and maturity of the Alliances. I identified three clusters of Alliances. First, there were Alliances that understood the concept, for example, the Canterbury Clinical Network. These Alliances had an agreed shared vision and common goals for their local system; were clinically led; had an independent chair and placed people and their communities at the centre of their decision-making. These Alliances were highly functional and successful in achieving their goals. The second cluster had some elements of the first cluster and were striving to do the right things but were constrained by their capacity and capability and lacked insight as to their strengths and weaknesses. The third cluster of Alliances were dysfunctional, either owing to a lack of leadership from the DHB and/or the PHO or a lack of understanding about the alliancing concept, and low-trust relationships between senior managers of DHBs and PHOs and between senior managers and clinicians in DHBs and PHOs. They were further hampered by the lack of capacity and capability for improvement and therefore lacked awareness of their inability to be functional.

The $\mathrm{MoH}$ required Alliances to use local clinically led quality improvement processes to implement the SLM programme $[15,40]$. Some districts used their existing clinical leadership forums or brought together clinical leaders from primary care and hospitals to examine data for the system level measures to determine the improvement focus and the frontline actions that would contribute to the improvement of those measures. In other places, implementation of the SLM programme brought together clinicians from primary care and hospitals. Previous efforts of working together significantly influenced discussions and where previous experiences had been unfavourable, discussions would focus on providers, professional groupings and funding. In 
contrast, where previous experiences had been successful, there was high-trust among clinicians and discussions were system- and people-focused.

Most DHBs had consumer councils, which were used to engage with patients and communities in the development and implementation of the SLM plans. There was a variable understanding among health providers about patient engagement and co-development or co-design with users of the health system and local communities. During the implementation of the primary care patient experience survey, some of the general practices were concerned that patients were going to use the survey to complain about waiting times or not being treated with respect and kindness. In few districts where there was both a genuine desire and the capacity and capability to deliver more patient-centred services, this was reflected in the Alliance membership and in their terms of reference. From my observations, conversations at these Alliances were peopleand system-focused.

In some cases, despite some DHBs' poor financial position and performance against accountability measures, success of the SLM programme implementation depended on DHBs and their Alliances' capacity and capability for improvement (i.e. people, information infrastructure and analytic capability). A few DHBs had continued to invest in developing improvement capacity and capability to understand their local health needs. Others focused on doing the minimum to meet the national accountability measures such as the national health targets.

Implementation of the SLM programme needed to be supported with adequate information technology infrastructure, which included DHBs' ability to join data sets and to turn data into insights that could be used for improvement. Implementation of the SLM programme showed high variability in the information technology capacity and capability of DHBs. DHBs that had invested in information technology infrastructure were able to join data from hospital, and primary and community care, study the patient journey through the health system, and understand how their demography influenced the demand for services throughout the system. They could identify patients and population groups that were either high users of the system or not receiving equitable care or were high users of hospital services because they were not receiving the right care in the right place at the right time. This enabled them to have patientand population-centred conversations with their clinicians and Alliance leadership teams to influence development of new models of care that would better meet the needs of the local population. Without this infrastructure and capacity, DHBs were severely hindered in the implementation of the SLM programme. 
The continuous improvement philosophy of the SLM programme required DHBs to regularly assess, review and prioritise health resources and consider new models of care to meet the health needs of the local population, referred to as 'commissioning cycles' [189]. Additionally, the Public Health and Disability Act 2000 mandates DHBs to undertake regular health needs assessments, which is then used to drive local commissioning of health services. My observations from leading the implementation of the SLM programme showed that the full commissioning cycle was not being used by DHBs in the development of the SLM plans. Most DHBs were using the annual planning guidance issued by the $\mathrm{MoH}$ that included Government priorities and the Minister of Health's expectations to develop their DHB annual plan. A few DHBs supplemented this guidance with information from their district health profiles that included demographic information and information on the health status of their populations. Alliances did not appear to be involved in the development of DHB annual plans and development of the SLM plans was occurring in parallel to the DHB annual planning process. The SLM plans required the signatures of Alliance members as a proxy to demonstrate an integrated approach, whereas, DHB annual plans did not have such a requirement. This parallel process disconnected implementation of the SLM programme from DHB planning processes.

Furthermore, most DHBs appeared to continue to purchase health services without a review process to establish if the services were meeting the health needs of their local populations. In some of the service level Alliance team meetings that I attended, middle managers and health care professionals would identify initiatives to target investment to address health inequities but did not know how to change the DHB contractual processes to implement these new initiatives. New models of care were emerging such as the Health Care Home and Integrated Family Health Centres, but these were mostly led by larger PHOs, with limited and variable investment in both funding and leadership from DHBs.

The disconnection between the DHB annual planning process and implementation of the SLM programme was also evident in the $\mathrm{MoH}$. The two processes sat under different business units of the $\mathrm{MoH}$, the rationale being that one was DHB focused while the SLM programme was wholeof-system focused. The $\mathrm{MoH}$ could not directly commission implementation of the SLM programme with Alliances nor hold them accountable because they were not legal entities. DHBs, as local commissioners of health services, remained accountable for submitting the SLM plan and quarterly reports to the $\mathrm{MoH}$ on behalf of Alliances as part of their DHB annual plan. The disconnection was exacerbated with $\mathrm{PHOs}$ receiving capacity and capability funding to support 
the implementation of the SLM programme, albeit this funding was re-purposed from the PHO Performance Programme. In meetings with the SLM programme clinical lead and I, DHB staff involved in implementation expressed that they felt responsible for implementation of the programme while PHOs received additional funding. From my observations, in districts where relationships between DHBs and PHOs were fraught, the disconnection led to the SLM programme being perceived as a primary care programme rather than a whole-of-system programme.

Table 9.1 shows the initial list of key elements gleaned from my insights gained from the implementation of the SLM programme.

\begin{tabular}{|l|}
\hline Key elements that supported successful implementation of the SLM programme \\
\hline An alliancing way of working \\
\hline Clinical leadership and engagement \\
\hline Use of commissioning cycles \\
\hline A continuous quality improvement focus \\
\hline Information and communication technology \\
\hline Community and patient engagement \\
\hline
\end{tabular}

Table 9.1: Initial list of key elements gleaned from my insights

\subsection{Literature review}

This phase involved reviewing published and grey literature on LST initiatives and identifying the key elements theorised to be essential to support these initiatives. An initial search revealed 10 studies that identified key elements and contextual factors that influenced implementation of a large-scale change. These are summarised in Appendix 2. Of these 10, five studies met the definition of LST initiatives used in this research and were reviewed in detail. While the remaining five were not considered to strictly meet the LST initiative definition, these were considered useful as these identified some nuggets of evidence on successful implementation of a largescale change.

\subsubsection{Study 1: Large-system transformation in health care: a realist review [1]}

This Canadian study was commissioned by the provincial Saskatchewan Ministry of Health, which sought a synthesis of knowledge about successful and less successful LST initiatives, to guide their four major system transformation initiatives. The Ministry convened a steering committee comprising senior representatives from the Ministry, regional health authorities and a provincial quality council. The steering committee provided oversight of the study and requested specific 
recommendations from researchers on ways the Ministry could support and sustain their transformation initiatives. The researchers used realist methodology, nested within a macro framing of complex adaptive systems, to identify the underlying mechanisms that influenced implementation of LST initiatives, taking into consideration the existing structural and policy contexts.

The study identified five 'simple rules' to enhance successful implementation of LST initiatives in health care: blend designated and distributed leadership; establish feedback loops; attend to history; engage physicians; and include patients and families. Along with the steering committee, the study also engaged with international experts to develop and describe the CMO theories to show variations in outcomes for LST initiatives. For example, if the system in question had a clearly identified mission, vision and values (context), then an explicit alignment of these by top and middle managers (mechanism) positively impacted on the blend of designated and distributed leadership (outcome). The study recommended a greater use of realist methodology that draws on both research theory and practice to better understand the role of contexts and mechanisms in the successful implementation of LST initiatives.

\subsubsection{Study 2: A system-wide transformation towards integrated care in the Basque Country: a realist evaluation [37]}

The Basque Country (Spain) introduced a system-wide transformation strategy to address the challenges from an increasing prevalence of chronic conditions with an ageing population and to transform the health system to overcome the fragmented delivery of care and to make the system sustainable. The implementation of the strategy involved multiple organisations and care providers across health and social care sectors. The study used a realist approach to gain relevant insights into how the strategy achieved its impact and to produce transferable lessons for decision-makers in other regions and countries. Using semi-structured interviews, the researchers analysed and described the CMO theories to explain the transformation phenomenon.

The study reported that system-wide transformation requires time, effort, leadership, vision and narrative, along with commitment, inclusiveness, collaborative decision-making processes and constant learning. Further, the study pointed out that key levers to integrate health and social care systems required developing new staff roles, investing in integrated electronic health records, stratifying the population by risk, and facilitating bottom-up innovations. The researchers concluded that LST initiatives require a systems approach and that it is almost 
impossible to show hard evidence of attribution in this systemic change; these transformation initiatives are 'humble exercises' of networking with many complex and unexpected factors, patterns and relationships that need to be carefully crafted, nurtured and developed.

\subsubsection{Study 3: Large-scale improvement initiatives in healthcare: a scan of the literature [30]}

The third study undertook a scan of the literature for large-system improvement initiatives in hospitals and health care systems to provide current thinking and practices for successful implementation of these initiatives. The reviewers used a modified Delphi technique with expert reviewers to review a total of 39 published articles. They used an iterative process to build consensus opinions among experts working in complex fields to generate meaningful and actionable information to support large-system improvement initiatives in health care. The study identified four primary and 16 secondary drivers for successful implementation of LST initiatives (Table 9.2). The reviewers recommended that these drivers be used as a 'checklist' for implementation of LST initiatives in health care.

\subsubsection{Study 4: Creating a high performing healthcare system for Ontario: evidence supporting strategic changes in Ontario [122]}

The fourth study reviewed international evidence on high performing health care systems, their characteristics and the key drivers together with insights from health system leaders in Ontario and patient experiences and expectations of the Ontario health care system. The latter was informed by focus groups and surveys with patients and caregivers, and a patient and caregiver expert panel. The researchers identified 12 key attributes of high performing health care system (Table 9.3). The study provided a framework to guide and support system-wide innovations to achieve the desired system transformation. 


\begin{tabular}{|c|c|}
\hline Primary drivers & Secondary drivers \\
\hline Planning and infrastructure & $\begin{array}{l}\text { Vision and aim } \\
\text { Intervention } \\
\text { Management } \\
\text { Resources } \\
\text { Other planning and infrastructure (e.g. information technology } \\
\text { capability) }\end{array}$ \\
\hline $\begin{array}{l}\text { Individual, Group, Organisational } \\
\text { and System factors }\end{array}$ & $\begin{array}{l}\text { Individual and group dynamics } \\
\text { Champions/change agents } \\
\text { Leadership roles } \\
\text { Capability and capacity development } \\
\text { Learning networks } \\
\text { Social networks } \\
\text { Organisational and system capability } \\
\text { Organisational and system culture }\end{array}$ \\
\hline The process of change & Change theory used \\
\hline $\begin{array}{l}\text { Performance measures and } \\
\text { evaluation }\end{array}$ & $\begin{array}{l}\text { Data infrastructure } \\
\text { Measurement and feedback systems }\end{array}$ \\
\hline
\end{tabular}

Table 9.2: Primary and secondary drivers for successful implementation of LST initiatives [30]

\section{Key attributes of high performing health care systems}

1. Focusing on quality and system improvement as the core strategy

2. Developing leadership skills

3. Enhancing system governance

4. Investing in capacity to support improvement

5. Improving accountability and performance measurement

6. Enabling comprehensive information infrastructures

7. Strengthening primary care

8. Improving integration and care transitions

9. Enhancing professional cultures and engaging clinicians

10. Engaging patients, caregivers and the public

11. Attending to access and equity issues

12. Considering population health and chronic disease management in care management strategies

Table 9.3: Key attributes of high performing health care systems [122]

\subsubsection{Study 5: Organisational context and capabilities for integrating care: a framework for improvement [3]}

The fifth study developed a framework of organisational capabilities to deliver integrated care and explored the mechanisms that influenced integrated care interventions. Integrated care interventions also build from theories that promote collaborative ways of working between 
diverse organisations and health care professionals to prompt change and achieve the desired system transformation [3].

The researchers reviewed 114 published articles that focused on integration at the system level, involving multiple sectors, organisations and professionals involved in the delivery of health care services. This was augmented with semi-structured interviews with organisational leaders and care providers involved in Ontario’s ‘Health Links' integrated care initiative.

The study identified 17 organisational contexts and capabilities categorised under basic structures, people and values, and key processes (Table 9.4). Of these, three capabilities that most shaped an organisation's capacity for integrated care were the leadership approach, clinician engagement and leadership, and a readiness for change. This study considered a large body of literature on integrated care and used the knowledge of those working in the health system to consolidate the key organisational capabilities into a framework for organisations to use to test their readiness for change.

\begin{tabular}{|l|l|}
\hline Category & Organisational context and capability \\
\hline Basic structures & Physical features \\
& Resources \\
& Accountability \\
Information technology \\
Organisational/Network design
\end{tabular}

Table 9.4: Organisational context and capabilities for integrating care [3]

Other studies have identified similar key elements essential for successful implementation of LST initiatives in health care, albeit on a smaller scale or limited to a profession or a part of the health system. These studies are summarised in Appendix 2. 
The five studies discussed above set the scene for my investigation into the key elements that had the greatest weight for a New Zealand context. These studies were chosen because they met the definition for LST initiatives used in this thesis.

Following a review of the literature, I compared my initial list of key elements with those identified in the five studies discussed above and made one change to my list; I separated the 'information and communication technology' element into two: integrated health information; and analytic capability.

LST initiatives require secure interoperable platforms that can collect and link patient-level data from all health service providers and support frontline clinicians in diagnosis and treatment of their patients. This is important for delivery of safe and co-ordinated care. Equally important is the ability to access, analyse, and link clinical and administrative data such as, socio-economic status to produce evidence for clinicians and leaders. This evidence helps with possible interventions and to track health outcomes, both at an identifiable and a population aggregate level. The evidence also provides feedback to clinicians with information on clinical variation that is important for continuous improvement of care provided and of professional practice $[30,122]$. The use of evidence can help in avoiding multiple and conflicting opinions, providing rigour to measurement and monitoring, and building trust and confidence between clinicians and management $[30,122,190]$. The integrated health information and analytic capability are two inter-linked elements that play an important role in implementation of LST initiatives, but require different skill sets and having one does not assume the presence of the other.

I recognised that there were elements mentioned in the literature that were not part of my initial list such as the leadership approach, the organisational culture, a readiness for change and the system governance. Given that these elements were more nuanced towards the organisational context and relevant to my third research question (contexts and mechanisms that influence implementation of LST initiatives), I chose to not include these in my list of key elements to be tested with the research participants. Instead, I provided this evidence from the literature to the workshop participants to consider as part of testing and refining the key elements essential for successful implementation of LST initiatives in New Zealand. Table 9.5 shows the revised list of key elements and descriptions that were prepared to be used for testing in the workshop and with the interviews. 
1. Alliancing way of Alliancing is a clinically led, community engaged forum that brings different working component parts of the health system together with the aim of transforming services so that they are best for the patient and best for the system. Alliancing is a way of working, reliant on trusted relationships and supported by enablers critical to continuous improvement. Successful Alliances bring alignment to multiple organisations and services present in a complex system, to achieve a common set of patient-centred goals. Alliancing is not about managing finances or organisational or professional accountabilities [43].

2. Clinical A system through which health care professionals provide leadership and leadership and system oversight with a focus on continuous quality improvement to create an engagement environment for evidence-based clinical practice and team-based approaches to care delivery [191].

3. Use of Commissioning is the process of continuously developing services and commissioning committing resources to enable the best health outcomes to be achieved for cycles individuals and the population, equity to be assured and experience enhanced within the resources available [189].

Strategic commissioning is the term used for all the activities involved in assessing and forecasting needs, links investment to agreed desired outcomes, considering options, planning the nature, range and quality of future services working in partnership to put these in place [192].

4. Integrated $\quad$ The availability and ease of use of health information, both at identifiable and health $\quad$ population aggregate level, across the different parts of the system at local and information national level. This will enable the clinicians to examine patient records as they treat patients, to share and compare information with colleagues and track patient outcomes [3,122].

5. Analytic Ability to access real-time information, analyse, link clinical and administrative capability data, and produce insights and evidence for frontline managers and health care professionals to measure, understand and feedback data on clinical variation and outcomes, which is a critical enabler to continuous quality improvement $[122,190]$.

6. Continuous The systemic use of methods and tools to continuously improve all three quality improvement focus dimensions of the NZ Triple Aim: population health, experience of care and cost per capita [36]. The philosophy of improvement is to improve incrementally from baseline and learning from experience while doing so through the plando-study-act cycle [139].

7. Engagement An approach that consciously adopts individuals', carers', families', and with patients communities' perspectives in the design and delivery of health care to generate and significant benefits to the health and health care of all people, including

communities improved access to care, improved health and clinical outcomes, better health literacy and self-care, and better experience and satisfaction with care [17]. 


\subsection{Interviews}

Semi-structured interviews were used to test the concepts from the literature on key elements that need to be present in complex adaptive systems to increase the chances of success with implementation of LST initiatives and explanations of how these elements work in different contexts. Table 9.6 shows how the interview participants working in the health system, either in New Zealand or internationally $(n=9)$ rated the revised list of seven key elements.

The ratings from non-health participants $(n=3)$ are presented separately in Table 9.7. This is because interviews for these participants were less structured to enable a freer conversation. The key elements and descriptions were shared with these participants prior to the interview to allow them thinking time to relate the research to their industry. I was able to undertake two of the interviews, from banking (IP10) and construction industries (IP11), with a similar structure to that of the health participants, albeit not all the key elements were rated by the participants. It was difficult to maintain the interview structure with the last interviewee (IP12) who was an expert in organisational change management. The conversation with this interviewee was more focused on the complex adaptive nature of the health system, and the contexts and mechanisms that influence system transformation rather than the individual elements.

For each element, participants were read out the description of the element and were then asked to rate the extent that they agreed or disagreed with the element being necessary to increase the chances of success with implementation of LST initiatives. Participants used a five-point Likert scale (strongly disagree, disagree, neutral, agree, and strongly agree) to rate the element. In some instances, participants chose to explain their rating, in others they did not. Once participants had finished rating the elements, they were asked to identify additional elements. Participants identified 11 additional elements, which are discussed later in the chapter.

Except for participant IP12, most participants either agreed or strongly agreed with the seven key elements in the revised list. Participants suggested changing the language for two of the elements: clinical leadership and engagement to clinical leadership and involvement; and engagement with patients and communities to involved people and communities. Participants felt that the change in language for both elements strengthened the concept. A new element (dedicated resources and time) that was identified by six participants, was added to the list. The next sections (9.3.1 - 9.3.8) discuss participants' feedback on the key elements in detail. 


\begin{tabular}{|c|c|c|c|c|c|c|c|}
\hline & $\begin{array}{l}\text { Alliancing } \\
\text { way of } \\
\text { working }\end{array}$ & $\begin{array}{c}\text { Clinical } \\
\text { leadership } \\
\text { and } \\
\text { engagement }\end{array}$ & $\begin{array}{l}\text { Use of } \\
\text { commission } \\
\text {-ing cycles }\end{array}$ & $\begin{array}{l}\text { Integrated } \\
\text { health } \\
\text { information }\end{array}$ & $\begin{array}{l}\text { Analytic } \\
\text { capability }\end{array}$ & $\begin{array}{l}\text { Continuous } \\
\text { Quality } \\
\text { Improvement } \\
\text { focus }\end{array}$ & $\begin{array}{c}\text { Engagement } \\
\text { with patients } \\
\text { and } \\
\text { communities }\end{array}$ \\
\hline IP01 & Agree & $\begin{array}{l}\text { Strongly } \\
\text { agree }\end{array}$ & Agree & $\begin{array}{l}\text { Strongly } \\
\text { agree }\end{array}$ & $\begin{array}{c}\text { Strongly } \\
\text { agree }\end{array}$ & $\begin{array}{l}\text { Strongly } \\
\text { agree }\end{array}$ & $\begin{array}{l}\text { Strongly } \\
\text { agree }\end{array}$ \\
\hline IP02 & $\begin{array}{l}\text { Strongly } \\
\text { agree }\end{array}$ & $\begin{array}{l}\text { Strongly } \\
\text { agree }\end{array}$ & Disagree & Agree & Agree & $\begin{array}{l}\text { Strongly } \\
\text { agree }\end{array}$ & $\begin{array}{l}\text { Strongly } \\
\text { agree }\end{array}$ \\
\hline IP03 & $\begin{array}{l}\text { Strongly } \\
\text { agree }\end{array}$ & $\begin{array}{l}\text { Strongly } \\
\text { agree }\end{array}$ & $\begin{array}{c}\text { Strongly } \\
\text { agree }\end{array}$ & $\begin{array}{l}\text { Strongly } \\
\text { agree }\end{array}$ & $\begin{array}{l}\text { Strongly } \\
\text { agree }\end{array}$ & $\begin{array}{l}\text { Strongly } \\
\text { agree }\end{array}$ & $\begin{array}{l}\text { Strongly } \\
\text { agree }\end{array}$ \\
\hline IP04 & $\begin{array}{l}\text { Strongly } \\
\text { agree }\end{array}$ & $\begin{array}{l}\text { Strongly } \\
\text { agree }\end{array}$ & $\begin{array}{c}\text { Strongly } \\
\text { agree }\end{array}$ & $\begin{array}{l}\text { Strongly } \\
\text { agree }\end{array}$ & $\begin{array}{c}\text { Strongly } \\
\text { agree }\end{array}$ & $\begin{array}{l}\text { Strongly } \\
\text { agree }\end{array}$ & $\begin{array}{c}\text { Strongly } \\
\text { agree }\end{array}$ \\
\hline IP05 & Agree & $\begin{array}{l}\text { Strongly } \\
\text { agree }\end{array}$ & $\begin{array}{l}\text { Strongly } \\
\text { agree }\end{array}$ & $\begin{array}{l}\text { Strongly } \\
\text { agree }\end{array}$ & $\begin{array}{l}\text { Strongly } \\
\text { agree }\end{array}$ & $\begin{array}{l}\text { Strongly } \\
\text { agree }\end{array}$ & $\begin{array}{l}\text { Strongly } \\
\text { agree }\end{array}$ \\
\hline IP06 & $\begin{array}{l}\text { Strongly } \\
\text { agree }\end{array}$ & Agree & $\begin{array}{l}\text { Strongly } \\
\text { agree }\end{array}$ & Agree & $\begin{array}{l}\text { Strongly } \\
\text { agree }\end{array}$ & $\begin{array}{l}\text { Strongly } \\
\text { agree }\end{array}$ & $\begin{array}{l}\text { Strongly } \\
\text { agree }\end{array}$ \\
\hline IP07 & Agree & $\begin{array}{l}\text { Strongly } \\
\text { agree }\end{array}$ & $\begin{array}{l}\text { Strongly } \\
\text { agree }\end{array}$ & $\begin{array}{l}\text { Strongly } \\
\text { agree }\end{array}$ & $\begin{array}{c}\text { Strongly } \\
\text { agree }\end{array}$ & $\begin{array}{l}\text { Strongly } \\
\text { agree }\end{array}$ & $\begin{array}{l}\text { Strongly } \\
\text { agree }\end{array}$ \\
\hline IP08 & $\begin{array}{l}\text { Strongly } \\
\text { agree }\end{array}$ & $\begin{array}{l}\text { Strongly } \\
\text { agree }\end{array}$ & Neutral & $\begin{array}{l}\text { Strongly } \\
\text { agree }\end{array}$ & $\begin{array}{l}\text { Strongly } \\
\text { agree }\end{array}$ & Neutral & $\begin{array}{l}\text { Strongly } \\
\text { agree }\end{array}$ \\
\hline IP09 & $\begin{array}{l}\text { Strongly } \\
\text { agree }\end{array}$ & $\begin{array}{l}\text { Strongly } \\
\text { agree }\end{array}$ & $\begin{array}{l}\text { Strongly } \\
\text { agree }\end{array}$ & $\begin{array}{l}\text { Strongly } \\
\text { agree }\end{array}$ & Agree & $\begin{array}{c}\text { Strongly } \\
\text { agree }\end{array}$ & $\begin{array}{l}\text { Strongly } \\
\text { agree }\end{array}$ \\
\hline
\end{tabular}

Table 9.6: Interview participants' ratings on revised list of key elements (health)

\begin{tabular}{|c|c|c|c|c|c|c|c|}
\hline & $\begin{array}{l}\text { Alliancing } \\
\text { way of } \\
\text { working }\end{array}$ & $\begin{array}{l}\text { Clinical } \\
\text { leadership } \\
\text { and } \\
\text { engagement }\end{array}$ & $\begin{array}{l}\text { Use of } \\
\text { commission } \\
\text {-ing cycles }\end{array}$ & $\begin{array}{l}\text { Integrated } \\
\text { health } \\
\text { information }\end{array}$ & $\begin{array}{l}\text { Analytic } \\
\text { capability }\end{array}$ & $\begin{array}{c}\text { Continuous } \\
\text { Quality } \\
\text { Improvement } \\
\text { focus }\end{array}$ & $\begin{array}{c}\text { Engagement } \\
\text { with } \\
\text { patients and } \\
\text { communities }\end{array}$ \\
\hline IP10 & $\begin{array}{l}\text { Strongly } \\
\text { agree }\end{array}$ & $\begin{array}{l}\text { Strongly } \\
\text { agree }\end{array}$ & $\begin{array}{c}\text { Not } \\
\text { answered } \\
\text { clearly }\end{array}$ & $\begin{array}{l}\text { Strongly } \\
\text { agree }\end{array}$ & $\begin{array}{l}\text { Strongly } \\
\text { agree }\end{array}$ & $\begin{array}{l}\text { Strongly } \\
\text { agree }\end{array}$ & Agree \\
\hline IP11 & $\begin{array}{l}\text { Strongly } \\
\text { agree }\end{array}$ & $\begin{array}{l}\text { Strongly } \\
\text { agree }\end{array}$ & $\begin{array}{l}\text { Strongly } \\
\text { agree }\end{array}$ & $\begin{array}{l}\text { Strongly } \\
\text { agree }\end{array}$ & $\begin{array}{l}\text { Strongly } \\
\text { agree }\end{array}$ & $\begin{array}{l}\text { Strongly } \\
\text { agree }\end{array}$ & $\begin{array}{c}\text { Not } \\
\text { answered } \\
\text { clearly }\end{array}$ \\
\hline IP12 & $\begin{array}{c}\text { Not } \\
\text { answered }\end{array}$ & $\begin{array}{c}\text { Not } \\
\text { answered }\end{array}$ & $\begin{array}{c}\text { Not } \\
\text { answered }\end{array}$ & $\begin{array}{c}\text { Not } \\
\text { answered }\end{array}$ & $\begin{array}{c}\text { Not } \\
\text { answere } \\
d\end{array}$ & $\begin{array}{c}\text { Not } \\
\text { answered }\end{array}$ & $\begin{array}{c}\text { Not } \\
\text { answered }\end{array}$ \\
\hline
\end{tabular}

Table 9.7: Interview participants' ratings on revised list of key elements (non-health)

\subsubsection{Alliancing way of working}

Alliancing is a collaborative way of working, reliant on trusted relationships and supported by enablers critical to continuous improvement.

Eight participants strongly agreed and three agreed that an alliancing way of working was a key element necessary for successful implementation of LST initiatives in the New Zealand health 
system. This included the two participants from the banking and construction industries, both of whom strongly agreed with IP10 saying that an alliancing way of working was the foundation for bringing various sectors and disciplines together to deliver on the project.

You've got to have everyone on the same page. You've got to have those Alliances with everyone and make sure that you talk as one voice, otherwise you're just going to end up on the wrong side of the road. (IP10: participant from construction industry)

One overseas participant (IP06) strongly agreed with the concept but stated they would use different language to describe the alliancing way of working such as "relational or networking". Participant IP09 from Australia said that international literature on integrated care advocates for an alliancing way of working to bring together the meso level organisations responsible for delivering health services in a geographical area such as with DHBs and PHOs.

Participants (IP02, IP04 and IP05) mentioned that alliancing is an "extremely powerful policy mechanism" and a lever for bringing together the key players in a region or district for LST initiatives. Participant IP03 said that an alliancing way of working provided a critical platform for health providers in a district or a region to come together, understand the local priorities, challenge current investment decisions and make recommendations about shifting the investment to deliver on the local health priorities.

Participant IP04 emphasised that for alliancing to work, it must be "place-based and close to the population served".

Findings from the interviews indicated that an alliancing way of working builds relationships that means agents in the system may speak with one voice on local health priorities. Further, this voice has more meaning when it is shaped by a collective sense of purpose or vision.

\subsubsection{Clinical leadership and engagement}

Of 11 participants who rated this element, 10 strongly agreed with this being a key element for successful implementation of LST initiatives in the New Zealand health system. This included the two non-health participants, IP10 and IP11, who both strongly agreed.

You have to have technical expertise in certain disciplines when it comes to especially engineering - mechanical engineering, construction engineering, so there's definitely conversations where you would need to pull these people in and 
have that experience and expertise highlighted, and they've got to bring that to the table. (IP10: non-health participant)

One participant (IP06) who 'agreed' (instead of strongly agreed) did so because they felt the language I had used to describe the element was not strong enough. This participant suggested that it needed to be more than engagement, that it should be involvement of clinical leaders as they are powerful influencers in the system.

But you do need to bring the clinicians with you and many of them can be powerful influencers if you can unlock that. (IP06)

Participants reinforced that clinical leadership and engagement was important at all phases of planning and implementation of LST initiatives.

One participant (IP07) went further to say that clinicians make most of the decisions in the health system around treatment and diagnostics, which is where most of the health care funding is spent. Often clinicians do not realise the power they have in the system and with support from senior operational leaders, they can be powerful influencers for their colleagues and for the patients.

A lack of clinical engagement in LST initiatives becomes a barrier to change and that "sometimes mooted agreement or mooted support is as good as you're ever going to get" (IP03) but this was enough when trying to bring different system agents on board. One participant (IP05) highlighted that this element is important from the perspective of patient safety, that clinical experience is needed in decision-making to ensure that any change is implemented safely.

I think it would be also decisions that are informed by clinicians in terms of if you're developing a particular program for diabetes let's say, then you need to have one is the clinical experience to understand how it's delivered in the frontline but also around having that clinical safety perspective, perhaps that might play a part too within such a program. (IP05)

Although the third non-health participant (IP12) did not rate the element, they gave strong feedback on the importance of having intrinsic domain knowledge of the system to nurture a continuous improvement culture.

So, knowledge of the areas so that you actually understand intrinsically how things are done in this business. So, we don't actually value domain knowledge, which 
means we don't actually value continuous improvement. So, we've got a cadre of leaders who actually have no understanding of either the systems they are supposed to be in charge of or of the importance of investing in continuous improvement. (IP12: non-health participant)

Participant (IP04) strongly agreed with this element but also highlighted that disciplines around management and implementation are also important for successful implementation of LST initiatives and that clinical leaders may not have these skills or the time to be involved. Therefore, a blended model that is clinically led with a manager alongside strengthens the team for transformation.

...probably a bit like your relationship with (SLM programme clinical lead), it's a stronger team because you've actually got both of those strengths together. (IP04)

Early findings show that this element is necessary for successful implementation of LST initiatives as clinical leaders have technical expertise to ensure that a change is not only safe but works in real world. Further, they are powerful agents in the system who can influence behaviour of their colleagues and their patients.

\subsubsection{Use of commissioning cycles}

This element had the most variable response with ratings spread across the spectrum of strongly agreed and disagreed. Seven participants strongly agreed, one agreed, one was neutral (IP08) and one disagreed (IP02). Two participants did not give a clear rating. Participant IP08 did not disagree with the concept but strongly believed that this, along with the 'continuous quality improvement' element needed to be continuous rather than static elements. The participant suggested that commissioning cycles included continuous quality improvement and that 'developmental evaluation' better described the commissioning process. Participant IP03 also said that commissioning was a continuous cycle and that it was an important tool and lever for system transformation.

If you're understanding where you want to invest or disinvest, or shift the emphasis, then commissioning is a cool tool that sits alongside that alliancing function. (IP03)

Another participant (IP05) highlighted the importance of commissioning for addressing health inequities as it provided the evidence to challenge the status quo or the institutional bias in the system. 
Participant IP02 disagreed with the element as this participant understood commissioning to be a contractual arrangement that did not support a collaborative way of working and therefore had a different ethos to that of an alliancing way of working and clinical leadership and engagement. Other participants, in particular IP03, described the lack of understanding or varied understanding of this element, especially between commissioning, accountability and contracting. Participants who agreed with this element emphasised the difference between commissioning and contracting. The latter is a pricing discussion while the former involves strategic planning to align investments to local health needs (IP03). Contracting is one part of a commissioning cycle.

I think commissioning is used almost as a catch-all for everything. Some people interpret it as what I'd call contract management. Renegotiating the annual contract for aged residential care is a pricing discussion. It's not commissioning. (IP03)

This is not about procurement and transactional contracts. This has to be much more around strategic planning and commissioning. (IP06)

Participant IP06 also said that the commissioning cycle needs to be given enough time to mature and to be allowed to deliver impact.

Probably a combination of short, sharp rapid cycle changes but with strategic commissioning over a decent time period. (IP06)

Participant IP07 said that the lack of understanding for this element was made worse with the separation of commissioning and contracting with service improvement and that the DHB planning and funding units, which are the hub service improvement, employ contract managers instead of those with improvement skills. The contract managers then try and manage service improvement through transactional processes.

In summary, feedback from participants highlighted variable understanding of the commissioning concept and generated rival ideas on how this element works to support successful implementation of LST initiatives. Participants did not offer an alternate description of this element. However, there was a strong link identified between this element and continuous quality improvement, which is discussed in the next section. 


\subsubsection{Continuous quality improvement focus}

Of 11 participants who rated this element, 10 strongly agreed that this element was key to supporting successful implementation of LST initiatives in the health system. Although participant IP12 did not rate the element, they believed that a continuous quality improvement focus, in the form of constant feedback loops, was at the core of system transformation in complex adaptive systems. The participant from the construction industry (IP10) said that continuous improvement was vital as this enabled businesses and organisations to keep up with the technology evolution.

Without continuing improvement, you set yourself up for disaster. Technology changes continuously. What you know today is outdated by tomorrow, so to not have continuous improvement in place would be disastrous for your business. (IP10: non-health participant)

Participant IP06 said that transformational change in health and social care is an improvement journey, that "it's implementation science in action".

One participant (IP08) rated this element neutral, not because they disagreed with the concept but because they strongly believed that this element was part of commissioning cycles (discussed earlier). The participant suggested that continuous improvement can be perceived as an "orderly kind of thing" when in fact "disruptive innovation" may be a better description of the element.

We tend to use developmental evaluation rather than a PDSA framework so that there's continuous improvement and revamping of the strategy, revamping of the innovation and so as you go forward. (IP08)

Another participant (IP03) suggested that this element should be named as "improvement focus" because an improvement focus leads to a thirst for open critique and challenge, which then steers the system towards delivery of high-quality care.

Participant IP09 said that for clinicians, continuous quality improvement "needs to be real time" unlike a randomised control trial where "you are waiting two years or three years to get the information". Continuous quality improvement process of plan-do-study-act should be embedded into the clinical practice.

Participant IP01 emphasised that continuous quality improvement does not displace the need for performance monitoring and quality assurance. The participant shared their experience of the early days of the SLM programme development when it was the Integrated Performance and 
Incentive Framework. The clinicians implicitly rejected any form of performance monitoring that was not quality improvement because they believed that "if it is not about quality improvement then it is not a legitimate thing to do". According to this participant, continuous quality improvement is part of the clinical leadership and engagement and an effective clinical engagement is unlikely in the absence of a quality improvement approach.

\subsubsection{Integrated health information}

Nine participants strongly agreed and two agreed with integrated health information being a key element necessary to support successful implementation of LST initiatives. Participants reinforced that this element is not just about having a single electronic platform for clinical information but having the ability to link datasets from multiple platforms that includes administrative and social sector data such as socio-economic status and social outcomes and at the patient identifiable level. Participants said the linked datasets provided the frontline health care professionals the evidence for system improvement.

But, our experience in Estonia and in some other places in Europe we've looked at is the benefit of data sharing really comes into its own when you start layering the social services data as well. (IP04)

I strongly agree with data to drive decision making because otherwise it's just anecdote. (IP07)

One participant (IP04) said that owing to current cyber environment, people are cautious about combining patient identifiable level information for analysis. The cautious approach makes data less useful for frontline health care professionals as they are not able to identify and respond to specific patient needs.

Unless you can serve the frontline with the information, you're using then you're creating more heat from light. (IP04)

The participant from the banking industry (IP11) mentioned their experience with organisations who want the data to be perfect before using it and said that they had "never come across any organisation that has perfect data".

Feedback from participants confirmed that presence of integrated health information is necessary to build evidence for change, establish baseline and to measure progress of LST initiatives, which is then fed back into the system, enabling implementers to adjust their improvement actions. 


\subsubsection{Analytic capability}

Nine participants strongly agreed and two agreed with analytic capability being a key element to support successful implementation of LST initiatives. Participants accentuated that this element complemented the previous element, integrated health information however, that having one did not mean presence of the other. Participants, both in New Zealand and overseas, highlighted there was a lack of analytic capacity and capability in the health care system. This was exacerbated by a lack of awareness by system leaders on their organisations' deficiency of the analytic capacity and capability.

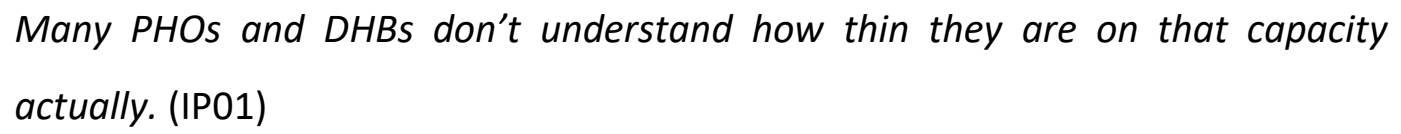

Most systems that I have engaged with in my own country and beyond are very data rich but analytic poor, analysis poor. (IP06)

I think there's a huge amount of data but the amount of business intelligence there and ability to analyse that and tell us something useful is often lacking. (IP07)

Participants expressed the important role that analytic capability plays in supporting some of the other elements such as alliancing way of working and using the evidence gained to engage and support health care professionals in continuous quality improvement.

Participant IP06 said that once system leaders recognise the value of analytic capability, it then enables them to share and pool the capacity and capability across the health system and increase their rigour of measuring progress to ensure that the transformation initiative is achieving its goals.

Some participants were of the view that having analytic capability was more important than having integrated health information, as those with the former found a way to link information from multiple sources: "if you've got really good intelligence function you can get people to knit stuff together" (IP03).

The participant from the banking industry (IP11) highlighted that analytic processes cannot solely rely on artificial intelligence or automation. Human intervention is needed to understand the insights from the data. This was reinforced by another participant (IP07) with the example of a chronic obstructive pulmonary disease (COPD) project in Christchurch following the earthquake. The data showed that most of the COPD cases were geospatially mapped to those living in the city centre. The project team used this information to begin designing the solution. Later in the 
project, a new member to the project highlighted that the city centre was cordoned off and that people had moved away so the data did not seem accurate. Further investigations revealed that patients who were not enrolled with a general practice were coded to the city centre by default. This highlighted the need for a local health intelligence function comprising technology and human analytic capabilities that is critical for LST initiatives.

Participant IP05 talked about the need for integrated health information and the analytic capability to use real-time and routinely collected data for operational and clinical performance measurement instead of relying on traditional clinical trials that do not necessarily provide the right information, take a long time to collect and are expensive to run.

Analytic capability, together with integrated health information, was identified as an important element that increased the chances of success with implementation of LST initiatives and that enabled implementers and leaders to measure progress against the transformation goals.

\subsubsection{Engagement with patients and communities}

All the participants who work in the health system $(n=9)$ strongly agreed with this element as being key to supporting successful implementation of LST initiatives in the New Zealand health system. Of the three non-health participants, one strongly agreed with this element while the other two did not provide a rating but explained why this element was important for system transformation.

Most participants said that the health system world-wide is finding this a challenge and are not involving patients, their carers, families and communities in designing the health services.

Getting patients involved would be very, very important - patients to actually help design the care process, a lot more carefully. At the moment I think we pay lip service to that whole concept. (IP02)

Participant IP03 said that while historically there has been engagement with patients around service design such as mental health and disability services, there is much more to be done at the governance level and involving patients and consumers in future planning and priority setting for the health system. The participant went on to say that those in the health system are not leveraging the trend of a "smart consumer" with the technology evolution to support patients and consumers get access to information and the models of care delivery that they want and "then use that as the mechanism to drive change back in the system. It's a very powerful thing". 
Participants also said that a shift of language away from 'patient' and 'engagement' is needed as these perpetuate a 'tick box' exercise of consulting patients once the decision is made. Participant IP04 said that the use of 'patient' tends to gravitate around health as opposed to health and social services. People and communities should be involved the governance and decision-making levels to co-design the system and services. Participant IP05 said that the system transformation to improve Māori health would come from co-designing of health services with Māori, hapū and Iwi.

I don't think engagement is strong enough. I think we don't do it very well and I think sometimes we play lip service to it and we get nominal patients or community meetings or something almost to tick a box. (IP06)

I think having not just patient engagement, but I think - because obviously carers and that whole community perspective, I think they add such a richness. (IP09)

Participant IP06 mentioned that "unless you start with the patient, family, caregivers, community at the heart then there's really no clear purpose for any of this" and that this element is a powerful integrator as it "brings people to the table that allows people to work together when they might have a different culture and a different value base".

The three participants from other industries supported the concept, albeit only one rated the element. While the other two did not rate the element, they talked about the importance of client/consumer engagement in the design of any system. Their main point was delivering a product that the customer wants instead of the experts deciding what the customer needs. They also highlighted the need to create appropriate governance and monitoring processes to ensure that the health system is meeting the needs of the consumers. For banking industry, where all banks offer similar products, the customer's choice relied on their experience with the bank. Therefore, the focus is to ensure that customers have consistent good experience with all parts of the bank, which fosters long-term relationship with and loyalty to the bank. For the construction industry, it is about delivering a product that the customer wants and is happy with. This means involving the customer in the beginning with the design of the home and understanding their needs. Participant IP12 talked about their experience in product design where the business wanted to improve productivity, but this was not the motivation for the customer. Failure to involve the customer from the beginning of the design process led to the end product being what the business wanted and not the customer. 
Participant IP11 (banking) emphasised the importance of having a workforce that reflects the community that is being served.

\subsubsection{Additional elements}

Table 9.8 shows additional elements identified by the interview participants. Additional elements were categorised using three themes: broad fit with an existing element; a new element; or an organisation context. Using these themes, one additional element was consistently identified by the participants, which was the importance of having dedicated resources and time to implement LST initiatives. Participant IP04 talked about the expectation of those sponsoring, leading or involved in LST initiatives to see change in a short-term cycle of six to 12 months and that a longterm view of five to 10 years was needed for system transformation. Participant IP06 said that system transformation needed to be nurtured with time and space.

Skills and the resources you need to do the change management-or the change in leadership. (IP01)

Give it time and space and stop interfering. You absolutely need to evaluate as you go. You know, monitor and continually tweak and evolve and adapt as you go but don't suffocate it with performance management, particularly in a short time scale. Give it oxygen and allow it to flourish. This is about nurturing transformational change. (IP06)

Adequate resources for change too, so there has to be a specific allocation of the necessary resources to innovate, to do things differently. Usually people are forced to try to do this from the corner of their desk and it doesn't work well at all. (IP08)

Table 9.8 shows the rest of the additional elements identified by the participants that were either mapped to the existing element of 'an alliancing way of working' or identified as organisational contexts that are discussed in the chapter 11. 


\begin{tabular}{|l|l|}
\hline Additional element & $\begin{array}{l}\text { Existing element, New element or } \\
\text { context }\end{array}$ \\
\hline Accountability & Organisational context \\
\hline Safe to fail - learning culture & Organisational context \\
\hline Clear vision and purpose x2 & Alliancing way of working \\
\hline Relationships - collaboration x2 & Alliancing way of working \\
\hline Clear understanding of roles across different levels & Organisational context \\
\hline Feedback loops & Organisational context \\
\hline Smart monitoring & Organisational context \\
\hline Time x2 & New element \\
\hline Developmental evaluation & Organisational context \\
\hline Adequate resources x4 & New element \\
\hline Supporting innovation & Organisational context \\
\hline
\end{tabular}

Table 9.8: Additional elements identified by interview participants

Table 9.9 shows how the key elements that increase the chances of success with implementation of LST initiatives were refined following the interviews. The change in language for the two elements strengthened the concepts: clinical leadership and engagement to clinical leadership and involvement; and engagement with patients and communities to involved people and communities. 


\begin{tabular}{|c|c|c|}
\hline $\begin{array}{l}\text { Key elements at the } \\
\text { beginning of interviews }\end{array}$ & $\begin{array}{l}\text { Key elements at the } \\
\text { conclusion of the } \\
\text { interviews }\end{array}$ & Change \\
\hline Alliancing way of working & Alliancing way of working & $\begin{array}{l}\text { No change as overall strongly supported by } \\
\text { the participants }\end{array}$ \\
\hline $\begin{array}{l}\text { Clinical leadership and } \\
\text { engagement }\end{array}$ & $\begin{array}{l}\text { Clinical leadership and } \\
\text { involvement }\end{array}$ & $\begin{array}{l}\text { Overall, strongly supported by the } \\
\text { participants. } \\
\text { Changed 'engagement' to 'involvement' to } \\
\text { reflect strong feedback on clinician } \\
\text { involvement in LST initiatives. }\end{array}$ \\
\hline $\begin{array}{l}\text { Use of commissioning } \\
\text { cycles }\end{array}$ & $\begin{array}{l}\text { Use of commissioning } \\
\text { cycles }\end{array}$ & $\begin{array}{l}\text { Overall, strongly supported by the } \\
\text { participants who understood the concept of } \\
\text { commissioning and what it involved. Links } \\
\text { identified to continuous quality } \\
\text { improvement but no alternate description } \\
\text { offered. }\end{array}$ \\
\hline $\begin{array}{l}\text { Integrated health } \\
\text { information }\end{array}$ & $\begin{array}{l}\text { Integrated health } \\
\text { information }\end{array}$ & $\begin{array}{l}\text { No change as overall strongly supported by } \\
\text { the participants }\end{array}$ \\
\hline Analytic capability & Analytic capability & $\begin{array}{l}\text { No change as overall strongly supported by } \\
\text { the participants }\end{array}$ \\
\hline $\begin{array}{l}\text { Continuous quality } \\
\text { improvement focus }\end{array}$ & $\begin{array}{l}\text { Continuous quality } \\
\text { improvement focus }\end{array}$ & $\begin{array}{l}\text { No change as overall strongly supported by } \\
\text { the participants }\end{array}$ \\
\hline \multirow[t]{2}{*}{$\begin{array}{l}\text { Engagement with } \\
\text { patients and communities }\end{array}$} & $\begin{array}{l}\text { Involved people and } \\
\text { communities }\end{array}$ & $\begin{array}{l}\text { Overall, strongly supported by the } \\
\text { participants. } \\
\text { Element reworded to reflect feedback from } \\
\text { the participants of the language shift needed } \\
\text { to strengthen the concept. }\end{array}$ \\
\hline & $\begin{array}{l}\text { Dedicated resources and } \\
\text { time }\end{array}$ & $\begin{array}{l}\text { New element identified by six participants: } \\
\text { four identified resources and two identified } \\
\text { the importance of time needed for system } \\
\text { transformations. }\end{array}$ \\
\hline
\end{tabular}

Table 9.9: Key elements before and after the interviews 


\subsection{Workshops}

The revised list of elements (Table 9.5) was tested with the workshop participants. Table 9.10 shows how the workshop participants $(n=9)$ rated the key elements individually at the beginning of the first workshop, prior to any group discussions. The participants had evidence from the literature review to support their decision-making. None of the participants disagreed or strongly disagreed with any of the elements tested. Eight out of nine participants either agreed or strongly agreed that all the proposed elements were necessary to increase the chances of success with implementation of LST initiatives.

\begin{tabular}{|c|c|c|c|c|c|c|c|}
\hline & $\begin{array}{l}\text { Alliancing } \\
\text { way of } \\
\text { working }\end{array}$ & $\begin{array}{c}\text { Clinical } \\
\text { leadership } \\
\text { and } \\
\text { engagement }\end{array}$ & $\begin{array}{l}\text { Use of } \\
\text { commissioning } \\
\text { cycles }\end{array}$ & $\begin{array}{l}\text { Integrated } \\
\text { health } \\
\text { information }\end{array}$ & $\begin{array}{l}\text { Analytic } \\
\text { capability }\end{array}$ & $\begin{array}{l}\text { Continuous } \\
\text { Quality } \\
\text { Improvement } \\
\text { focus }\end{array}$ & $\begin{array}{l}\text { Engagement } \\
\text { with } \\
\text { patients and } \\
\text { communities }\end{array}$ \\
\hline WP01 & $\begin{array}{l}\text { Strongly } \\
\text { agree }\end{array}$ & $\begin{array}{l}\text { Strongly } \\
\text { agree }\end{array}$ & Agree & $\begin{array}{l}\text { Strongly } \\
\text { agree }\end{array}$ & $\begin{array}{l}\text { Strongly } \\
\text { agree }\end{array}$ & Agree & $\begin{array}{l}\text { Strongly } \\
\text { agree }\end{array}$ \\
\hline WP02 & $\begin{array}{l}\text { Strongly } \\
\text { agree }\end{array}$ & $\begin{array}{l}\text { Strongly } \\
\text { agree }\end{array}$ & Agree & $\begin{array}{l}\text { Strongly } \\
\text { agree }\end{array}$ & Agree & Agree & Agree \\
\hline WP03 & $\begin{array}{l}\text { Strongly } \\
\text { agree }\end{array}$ & $\begin{array}{l}\text { Strongly } \\
\text { agree }\end{array}$ & Agree & $\begin{array}{l}\text { Strongly } \\
\text { agree }\end{array}$ & $\begin{array}{l}\text { Strongly } \\
\text { agree }\end{array}$ & Agree & Agree \\
\hline WP04 & $\begin{array}{l}\text { Strongly } \\
\text { agree }\end{array}$ & $\begin{array}{l}\text { Strongly } \\
\text { agree }\end{array}$ & Strongly agree & $\begin{array}{l}\text { Strongly } \\
\text { agree }\end{array}$ & $\begin{array}{l}\text { Strongly } \\
\text { agree }\end{array}$ & $\begin{array}{l}\text { Strongly } \\
\text { agree }\end{array}$ & $\begin{array}{l}\text { Strongly } \\
\text { agree }\end{array}$ \\
\hline WP05 & $\begin{array}{l}\text { Strongly } \\
\text { agree }\end{array}$ & $\begin{array}{l}\text { Strongly } \\
\text { agree }\end{array}$ & Agree & $\begin{array}{l}\text { Strongly } \\
\text { agree }\end{array}$ & $\begin{array}{l}\text { Strongly } \\
\text { agree }\end{array}$ & $\begin{array}{c}\text { Strongly } \\
\text { agree }\end{array}$ & Agree \\
\hline WP06 & $\begin{array}{l}\text { Neutral- } \\
\text { Agree }\end{array}$ & $\begin{array}{l}\text { Agree- } \\
\text { Strongly } \\
\text { agree }\end{array}$ & Neutral-Agree & $\begin{array}{l}\text { Strongly } \\
\text { agree }\end{array}$ & $\begin{array}{l}\text { Agree- } \\
\text { Strongly } \\
\text { agree }\end{array}$ & Did not rate & $\begin{array}{l}\text { Strongly } \\
\text { agree }\end{array}$ \\
\hline WP07 & $\begin{array}{l}\text { Strongly } \\
\text { agree }\end{array}$ & $\begin{array}{l}\text { Strongly } \\
\text { agree }\end{array}$ & Agree & $\begin{array}{l}\text { Strongly } \\
\text { agree }\end{array}$ & $\begin{array}{l}\text { Strongly } \\
\text { agree }\end{array}$ & $\begin{array}{l}\text { Strongly } \\
\text { agree }\end{array}$ & Agree \\
\hline WP08 & $\begin{array}{l}\text { Strongly } \\
\text { agree }\end{array}$ & $\begin{array}{l}\text { Strongly } \\
\text { agree }\end{array}$ & Agree & Agree & $\begin{array}{l}\text { Strongly } \\
\text { agree }\end{array}$ & $\begin{array}{l}\text { Strongly } \\
\text { agree }\end{array}$ & $\begin{array}{l}\text { Strongly } \\
\text { agree }\end{array}$ \\
\hline WP09 & Agree & Agree & Agree & $\begin{array}{l}\text { Strongly } \\
\text { agree }\end{array}$ & $\begin{array}{l}\text { Strongly } \\
\text { agree }\end{array}$ & Agree & Agree \\
\hline
\end{tabular}

Table 9.10: Workshop participant ratings on the revised list of key elements

\subsubsection{Feedback on key elements}

One participant (WP06) rated neutral for two of the elements: an alliancing way of working and the use of commissioning cycles; not because they disagreed with the elements but because of the language I had used to describe these. This participant also did not give a rating for continuous quality improvement focus because they thought that the culture and ability to undertake continuous quality improvement was more important than just having a focus. Discussions during the workshop agreed with this feedback and participants recommended changing the description of this element to continuous improvement. 
There was a strong agreement among participants that an alliancing way of working was the fundamental element for successful implementation of LST initiatives and that this needed to be operationally embedded in the DHB, understood and resourced adequately, and trusted to make the right decision on behalf of the system agents.

The Alliance isn't just a monthly meeting or six-weekly meeting and I think that's the key part here, is the alliancing does become a way of working for all the punters across the system.

The fact that it needs to be structurally embedded in the DHB and then it has to be resourced and planned with long term view.

Participants said that an alliancing way of working reinforced the collaborative way of working and a move away from the competitive models. It provided a framework to meet the government procurement rules that was acceptable to the Commerce Commission. An alliancing way of working also enabled the district to develop and agree the shared vision and goals, shared measurement, continuous communication and an integrated work programme.

That's where the collective impact methodology is quite useful if you line it up with the alliancing way of working, so that is about having the shared common purpose, shared measurement, mutually reinforcing activities, continuous communication and the back-bone function, which is all the things we've been talking about today.

There was a significant discussion on the use of commissioning cycles and how this element was described. Individually this element was not rated as strongly as some of the others. Discussion at the workshop highlighted the varying ways of understanding this element. However, once a common understanding was reached through the consensus building group activities, there was a strong agreement on this element. Participants recommended this element was reworded to intelligent commissioning to reflect the use of data and evidence for investment decisions and provided feedback on the description to make it clear and concise.

I've got intelligent planning in evidence and data to allocate resources. The other word we've got is that one, which is agility.

Data and evidence informed resource allocation.

Contracts and transactions. All of those processes drive competition, not collaboration. If you want to have collaboration, then you need to have open transparent data and evidence informed resource allocation. 
Participants agreed that integrated health information and analytic capability were important but different elements and that both were essential.

The analytic capability is key for working out what the needs are that drive your resourcing.

There was also a significant discussion on the element, engagement with patients and communities. Individual ratings were not very strong for this element. Those who agreed, as opposed to strongly agree, believed that this should be part of an alliancing way of working rather than a separate element.

Whether it's a separate thing or whether it needs to be up there and with the whole business of alliancing and getting everyone who's a key stakeholder involved.

Alliancing way of working was seen as being inclusive of all agents in the system, including those who use the system. However, concerns were also raised from participants that not all Alliances currently have collective perspectives of communities, that is, the Alliance may have one person representing a large community or several communities. For example, there may be one Pacific person on the Alliance representing all Pacific communities. Even those that have community representation, the views are not actually influencing the decision-making of the Alliance. Participants also emphasised that Alliances should not just be looking after patients; they should be looking after their population, especially those that experience disparity in the health system. Following discussion, consensus was reached to retain engagement with patients and communities as a separate element but reword to have a broader than patient focus and included population and whānau.

\subsubsection{Additional elements}

Table 9.11 shows the additional elements identified by participants. Additional elements were categorised using three themes: broad fit with an existing element; a new element; or an organisation context. There was unanimous agreement among participants on three new elements: a commitment to Te Tiriti o Waitangi; an understanding of equity; and dedicated resources and time.

I mean, equity should actually underpin all of these from our view, but maybe it needs to be specific.

And Treaty also, which is a related but different concept and one that's probably important for us to capture for a New Zealand tool. 
It's resourcing the process. It's deliberately resourcing the process rather than having everybody try and do it as part of their day job.

That's one of the reasons why it needs its own program office function because the evidence around an Alliance or a collaborative model of any kind is internationally now is if you don't actually have that program office function nothing happens. You need someone navigating the multiple organisation space whose job it is to do that.

\begin{tabular}{|c|c|}
\hline Additional element & Existing element, New element or context \\
\hline Trust $\times 2$ & Alliancing way of working \\
\hline Clear vision $\times 3$ & Alliancing way of working \\
\hline Cross-sector alliancing & Alliancing way of working \\
\hline Situational governance and system design & Organisational context \\
\hline CQI capability & Continuous improvement and analytic capability \\
\hline Collaboration between services & Alliancing way of working \\
\hline $\begin{array}{l}\text { Stronger voice from vulnerable population } \\
\text { groups }\end{array}$ & $\begin{array}{l}\text { New elements: } \\
\text { Understanding of equity } \\
\text { Commitment to Te Tiriti o Waitangi }\end{array}$ \\
\hline $\begin{array}{l}\text { Equity between clinical and management } \\
\text { leadership }\end{array}$ & Organisational context \\
\hline Community aspirations & Organisational context \\
\hline Shared sense of purpose & Alliancing way of working \\
\hline Aligned values and principles & Alliancing way of working \\
\hline Health system wellness plan & $\begin{array}{l}\text { New element: } \\
\text { Dedicated resources and time }\end{array}$ \\
\hline Politics & Organisational context \\
\hline Understanding between equity and equality & $\begin{array}{l}\text { New elements: } \\
\text { Understanding of equity } \\
\text { Commitment to Te Tiriti o Waitangi }\end{array}$ \\
\hline Succession planning & Organisational context \\
\hline Emphasis on wider determinants & Organisational context \\
\hline Maturity of the alliancing leadership team & Organisational context \\
\hline $\begin{array}{l}\text { Relationship, trust between organisations, } \\
\text { service providers, communities/patients }\end{array}$ & $\begin{array}{l}\text { Alliancing way of working } \\
\text { Engaged people, whanau and community } \\
\text { New element: } \\
\text { Understanding of equity } \\
\text { Commitment to Te Tiriti o Waitangi }\end{array}$ \\
\hline
\end{tabular}


Following facilitation activities in the workshop, participants reached group consensus on 10 key elements that is necessary in the New Zealand health system to support successful implementation of LST initiatives (Table 9.12). Workshop participants provided feedback on descriptions of the elements.

\begin{tabular}{|l|l|l|}
\hline $\begin{array}{l}\text { Key elements at the beginning of } \\
\text { the workshop }\end{array}$ & $\begin{array}{l}\text { Key elements at the conclusion } \\
\text { of workshop one }\end{array}$ & Change \\
\hline Alliancing way of working & Alliancing way of working & $\begin{array}{l}\text { Includes collaborative way of } \\
\text { working }\end{array}$ \\
\hline Clinical leadership and engagement & $\begin{array}{l}\text { Clinical leadership and } \\
\text { engagement }\end{array}$ & No change \\
\hline Use of commissioning cycles & Intelligent commissioning & Reworded \\
\hline Integrated health information & Integrated health information & No change \\
\hline $\begin{array}{l}\text { Analytic capability } \\
\text { fontinuous quality improvement }\end{array}$ & $\begin{array}{l}\text { Analytic capability } \\
\text { Continuous improvement }\end{array}$ & No change \\
\hline $\begin{array}{l}\text { Engagement with patients and } \\
\text { communities }\end{array}$ & $\begin{array}{l}\text { Engaged people, whanau and } \\
\text { community }\end{array}$ & Reworded \\
\hline & Dedicated resources and time & New element \\
\hline & $\begin{array}{l}\text { Commitment to Te Tiriti o } \\
\text { Waitangi }\end{array}$ & New element \\
\hline & Understanding of equity & New element \\
\hline
\end{tabular}

Table 9.12: Workshop participants' consensus on the key elements

Following the workshop, I finalised the key elements and their descriptions using the interview and workshop findings, as shown in Table 9.13. This was shared with workshop participants following the workshop to seek further feedback. No further feedback was received from the participants. This revised list was then used to construct the online survey. 
1. Alliancing way Alliancing is a clinically led, community engaged forum that brings of working different component parts of the health system together with the aim of transforming services so that they are best for the patient and best for the system. Alliancing is a collaborative way of working, reliant on trusted relationships and supported by enablers critical to continuous improvement. Successful Alliances bring alignment to multiple organisations and services present in a complex system, to achieve a common set of patient-centred goals. Alliancing is not about managing finances or organisational or professional accountabilities [43].

2. Commitment Was not identified as a key element

to Te Tiriti o

Waitangi

3. Clinical

leadership and

involvement

A system through which health care professionals provide leadership and system oversight with a focus on continuous quality improvement to create an environment for evidence-based clinical practice and teambased approaches to care delivery [191].

4. Engaged people, whānau and community

5. Integrated health information

An approach that consciously adopts individuals', carers', families', and communities' perspectives in the design and delivery of health care to generate significant benefits to the health and health care of all people, including improved access to care, improved health and clinical outcomes, better health literacy and self-care, and better experience and satisfaction with care [17].

The availability and ease of use of health information, both at identifiable and population aggregate level, across the different parts of the system at local and national level. This will enable the clinicians to examine patient records as they treat patients, to share and compare information with colleagues and track patient outcomes [3,122].

6. Analytic capability

Ability to access real-time and trend information, analyse, link clinical and administrative data, and produce insights and evidence for frontline managers and health care professionals to measure, understand and feedback data on clinical variation and outcomes, which is a critical enabler to continuous quality improvement $[122,190]$.
Alliancing is a clinically, community and Iwi-led forum that brings all agents in the health system together with the aim of transforming services with people and equity at the centre of their decision-making. Alliancing is a collaborative way of working, reliant on trusted relationships and supported by enablers critical to continuous improvement. Successful Alliances serve the interest of the community while preserving the autonomy of multiple organisations and services present in a complex system.

Recognising the principles of the Te Tiriti o Waitangi through equity and active protection to achieve equitable health outcomes for Māori; culturally appropriate health care that recognises and supports Māori models of care, and working in partnership with Māori in the governance, design, delivery and monitoring of health services [101]

A system through which health care professionals provide leadership and system oversight with a focus on continuous quality improvement to create an environment for evidence-based clinical practice and team-based approaches to care delivery [191]

An approach that actively involves individuals, carers, hapū, whānau, Iwi and communities in the design and delivery of health care to generate significant benefits to the health care and wellbeing of all people.

The availability of technology, and health and social information, both at identifiable and population aggregate level, across the different parts of the system at local and national level. This readily accessible health information is responsive to needs and guides commissioning decisions.

The availability of technology and the ability to access real-time and trend information, analyse, link clinical and administrative data, and produce insights and evidence for frontline staff to measure, understand and feedback data on clinical variation and outcomes. 


\section{Dedicated \\ Was not identified as a key element} resources and time

8. Intelligent commissioning

Commissioning is the process of continuously developing services and committing resources to enable the best health outcomes to be achieved for individuals and the population, equity to be assured and experience enhanced within the resources available. It includes many activities ranging from health needs assessment and development of clinical pathways to service specification and contract management or procurement, underpinned by continuous improvement [189].

9. Understanding Was not identified as a key element

of equity

10. Continuous improvement

The systemic use of methods and tools to continuously improve all three dimensions of the NZ Triple Aim: population health, experience of care and cost per capita $[36,133]$. The philosophy of improvement is to improve incrementally from baseline and learning from experience while doing so through the plan-do-study-act cycle [139].
Availability of appropriate continuous resourcing such as appropriate health workforce, funding, knowledge, time, project management support, and administration support. There is an acknowledgement that transformational change needs capacity and long-term commitment.

Commissioning is the process of continuously developing services and committing resources to enable the best health outcomes and wellbeing and that includes many activities ranging from health needs assessment, cultural paradigms and development of pathways to service specification and contract management or procurement, underpinned by continuous improvement.

Equity recognises different people with different levels of advantage require different approaches and resources to get equitable health outcomes [193]. Emphasis is given to Pacific and other high opportunity populations such as those with mental health conditions and those with disability.

Systemic and sustained use of continuous quality improvement methods, measurement tools and feedback loops that provide opportunities for learning and build accountability in the system.

Table 9.13: Consolidation of key elements and descriptions following interviews and workshops 


\subsection{Online survey}

The survey was informed by findings from the workshops and the interviews. The purpose of the survey was to further test the programme architecture for successful implementation of LST initiatives with those involved in the implementation of the SLM programme in the DHBs and the PHOs. The survey participants were different from those interviewed or that participated in the workshops. These participants included DHB leads for the SLM programme, PHO Chief Executives, middle managers and frontline staff who were involved in the development of the SLM plan; they determined the improvement actions, selected contributory measures, and measured and reported progress against the plan to the Alliance. Therefore, they had an inside knowledge and experience of what influenced the implementation of the SLM programme in their districts.

Fifty-one respondents participated in the online survey: 22 from DHBs, 28 from PHOs and one that did not identify them self as a DHB or PHO employee (Figure 9.2). These were made up of health care professionals $(n=8)$, those in management or leadership roles $(n=33)$, and others such as quality improvement leaders and analysts $(n=10)$; as shown in Figure 9.3.

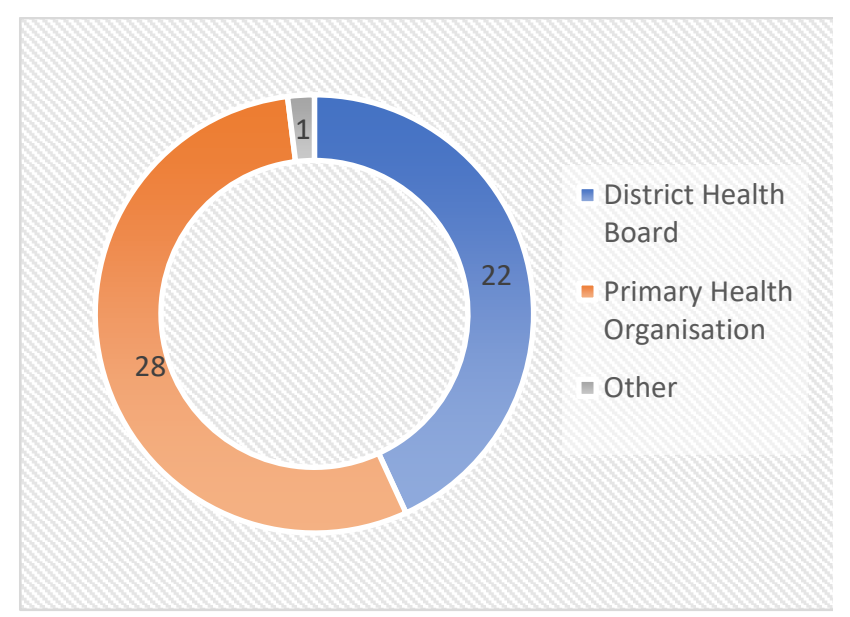

Figure 9.2: Survey respondents

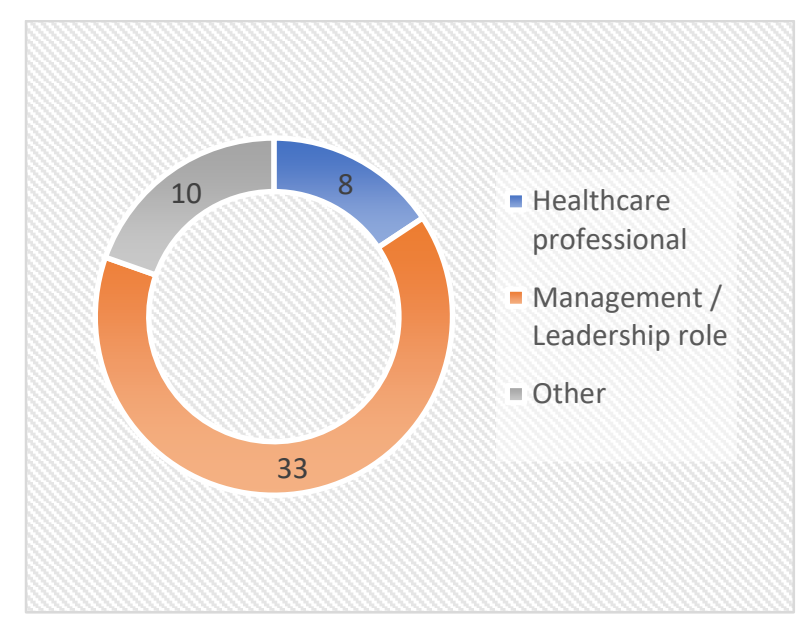

Figure 9.3: Role of respondents

\subsubsection{Ratings on key elements}

For each element, participants were given the description of the element and were then asked to rate; using a five-point Likert scale (strongly disagree, disagree, neutral, agree, and strongly agree) the extent that they agreed or disagreed with the element being necessary to implement LST initiatives successfully. Table 9.14 and Figure 9.4 show how participants rated on the key elements. The majority of the participants either agreed or strongly agreed with the 10 key elements. 
None of the participants disagreed on two of the elements: an alliancing way of working, and clinical leadership and involvement. Three participants indicated a neutral rating for these elements. The remaining $(n=48)$ either agreed or strongly agreed for these two elements.

There were three elements which participants rated neutral: involved people, whānau and community $(n=5)$; intelligent commissioning $(n=6)$; and dedicated resources and time $(n=4)$.

\begin{tabular}{|c|c|c|c|c|c|c|}
\hline Key element & $\begin{array}{l}\text { Strongly } \\
\text { disagree }\end{array}$ & Disagree & $\begin{array}{l}\text { Neither } \\
\text { agree } \\
\text { nor } \\
\text { disagree }\end{array}$ & Agree & $\begin{array}{l}\text { Strongly } \\
\text { agree }\end{array}$ & Total \\
\hline Alliancing way of working & 0 & 0 & 3 & 19 & 29 & 51 \\
\hline Commitment to Te Tiriti o Waitangi & 1 & 1 & 1 & 23 & 25 & 51 \\
\hline Understanding of equity & 1 & 0 & 0 & 16 & 34 & 51 \\
\hline Clinical leadership and involvement & 0 & 0 & 3 & 22 & 26 & 51 \\
\hline $\begin{array}{l}\text { Involved people, whānau and } \\
\text { community }\end{array}$ & 1 & 2 & 5 & 18 & 25 & 51 \\
\hline Intelligent commissioning & 0 & 1 & 6 & 16 & 27 & 50 \\
\hline Integrated health information & 0 & 2 & 2 & 12 & 35 & 51 \\
\hline Analytic capability & 1 & 1 & 3 & 12 & 34 & 51 \\
\hline Continuous improvement & 0 & 3 & 1 & 20 & 26 & 50 \\
\hline Dedicated resources and time & 1 & 4 & 4 & 10 & 31 & 50 \\
\hline
\end{tabular}

Table 9.14: Survey participants ratings on key elements

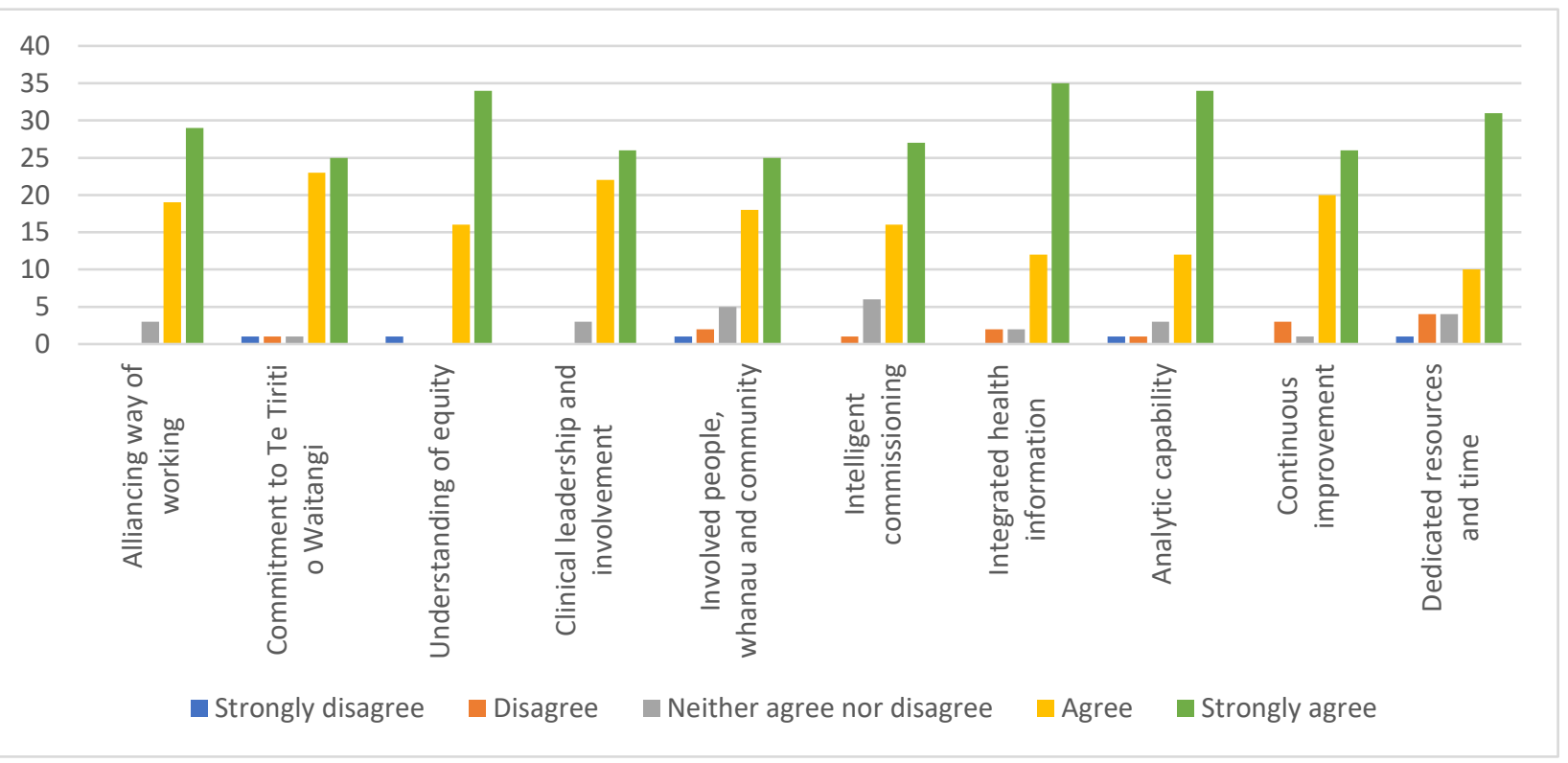

Figure 9.4: Survey participants' ratings on key elements 
There were two participants who were outliers in their rating of the key elements. The first, who worked in a PHO, strongly disagreed with four elements: commitment to Te Tiriti o Waitangi; understanding of equity; involved people, whānau and community; dedicated resources and time; and disagreed with continuous improvement. This participant agreed with an alliancing way of working; clinical leadership and involvement; and integrated health information and remained neutral for intelligent commissioning and analytic capability. The participant did not provide any reasons for their strong disagreement of the elements in their final comments.

The second participant was from a DHB who disagreed with six elements: involved people, whānau and community; intelligent commissioning; integrated health information; analytic capability; continuous improvement; and dedicated resources and time. The participant agreed with only one element: understanding of equity with a neutral rating on the remaining three. This participant also did not provide any reasons for their disagreement of the elements in their final comments.

When the results of agreed and strongly agreed are combined, it shows compelling evidence in support of the ten key elements tested with the participants (Figure 9.5).

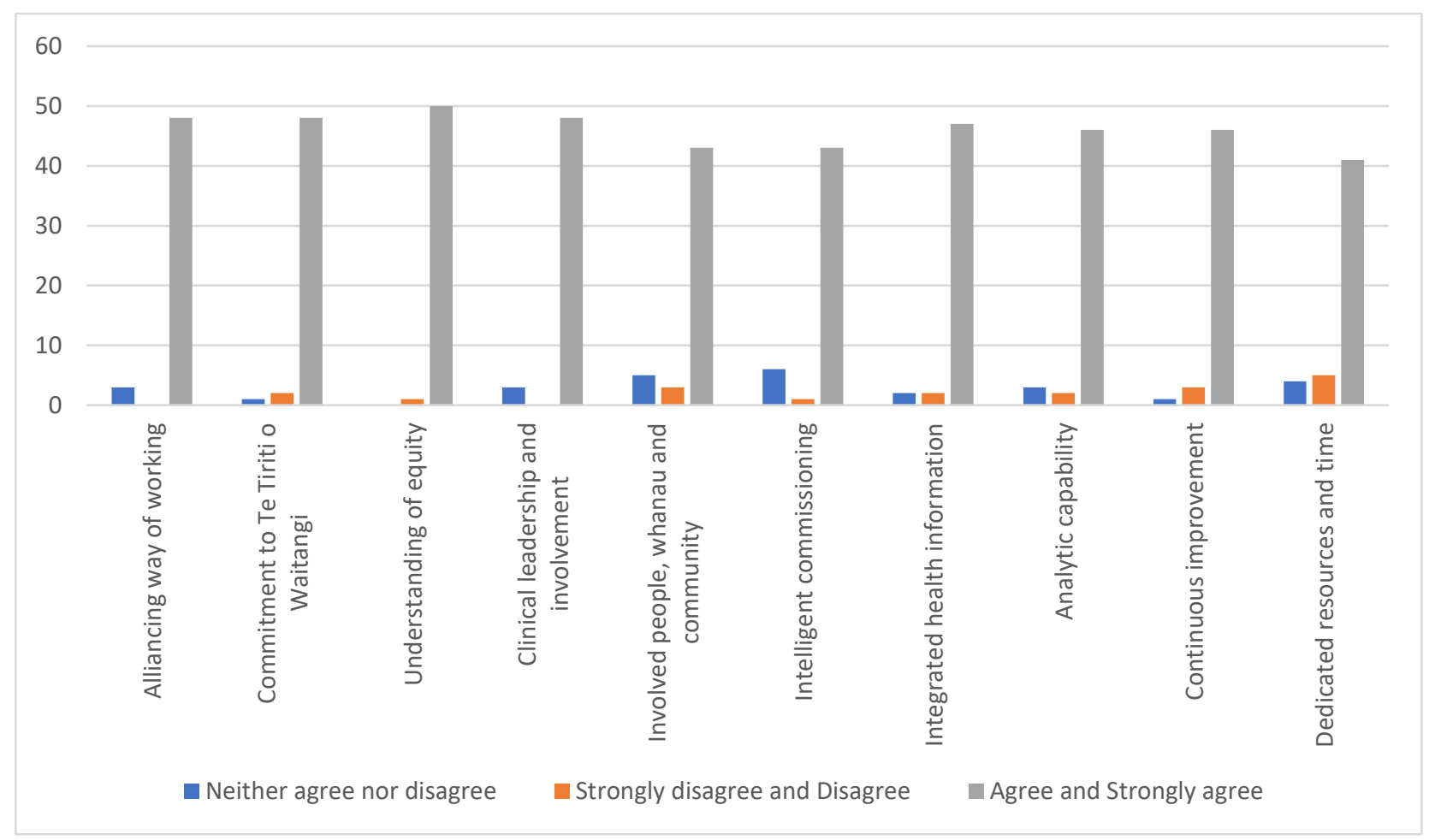

Figure 9.5: Ratings with combined scales 


\subsection{Chapter conclusion}

Table 9.15 shows the consolidation of key elements from initial theories gleaned from the SLM programme, literature review, interviews, workshop and the online survey. My research findings identified 10 key elements that are needed to increase the chances of success with implementation of LST initiatives in the New Zealand health system.

The next chapter presents the self-assessment maturity matrix that was developed with the senior leaders from the New Zealand health system.

\begin{tabular}{|c|c|c|c|c|}
\hline Initial theory & $\begin{array}{l}\text { After literature } \\
\text { review }\end{array}$ & After interviews & After workshop & Final list \\
\hline $\begin{array}{l}\text { Alliancing way of } \\
\text { working }\end{array}$ & $\begin{array}{l}\text { Alliancing way of } \\
\text { working }\end{array}$ & $\begin{array}{l}\text { Alliancing way of } \\
\text { working }\end{array}$ & $\begin{array}{l}\text { Alliancing way of } \\
\text { working }\end{array}$ & $\begin{array}{l}\text { Alliancing way of } \\
\text { working }\end{array}$ \\
\hline $\begin{array}{l}\text { Clinical leadership } \\
\text { and engagement }\end{array}$ & $\begin{array}{l}\text { Clinical leadership } \\
\text { and engagement }\end{array}$ & $\begin{array}{l}\text { Clinical leadership } \\
\text { and involvement }\end{array}$ & $\begin{array}{l}\text { Clinical leadership } \\
\text { and engagement }\end{array}$ & $\begin{array}{l}\text { Clinical leadership } \\
\text { and involvement }\end{array}$ \\
\hline $\begin{array}{l}\text { Use of } \\
\text { commissioning } \\
\text { cycles }\end{array}$ & $\begin{array}{l}\text { Use of } \\
\text { commissioning } \\
\text { cycles }\end{array}$ & $\begin{array}{l}\text { Use of } \\
\text { commissioning } \\
\text { cycles }\end{array}$ & $\begin{array}{l}\text { Intelligent } \\
\text { commissioning }\end{array}$ & $\begin{array}{l}\text { Intelligent } \\
\text { commissioning }\end{array}$ \\
\hline $\begin{array}{l}\text { Continuous quality } \\
\text { improvement focus }\end{array}$ & $\begin{array}{l}\text { Continuous quality } \\
\text { improvement } \\
\text { focus }\end{array}$ & $\begin{array}{l}\text { Continuous quality } \\
\text { improvement } \\
\text { focus }\end{array}$ & $\begin{array}{l}\text { Continuous } \\
\text { improvement }\end{array}$ & $\begin{array}{l}\text { Continuous } \\
\text { improvement }\end{array}$ \\
\hline \multirow{2}{*}{$\begin{array}{l}\text { Information and } \\
\text { communication } \\
\text { technology }\end{array}$} & $\begin{array}{l}\text { Integrated health } \\
\text { information }\end{array}$ & $\begin{array}{l}\text { Integrated health } \\
\text { information }\end{array}$ & $\begin{array}{l}\text { Integrated health } \\
\text { information }\end{array}$ & $\begin{array}{l}\text { Integrated health } \\
\text { information }\end{array}$ \\
\hline & Analytic capability & Analytic capability & Analytic capability & Analytic capability \\
\hline \multirow[t]{4}{*}{$\begin{array}{l}\text { Community and } \\
\text { patient } \\
\text { engagement }\end{array}$} & $\begin{array}{l}\text { Engagement with } \\
\text { patients and } \\
\text { communities }\end{array}$ & $\begin{array}{l}\text { Involved people } \\
\text { and communities }\end{array}$ & $\begin{array}{l}\text { Engaged people, } \\
\text { whānau and } \\
\text { community }\end{array}$ & $\begin{array}{l}\text { Involved people, } \\
\text { whānau and } \\
\text { community }\end{array}$ \\
\hline & & $\begin{array}{l}\text { Dedicated } \\
\text { resources and } \\
\text { time }\end{array}$ & $\begin{array}{l}\text { Dedicated } \\
\text { resources and } \\
\text { time }\end{array}$ & $\begin{array}{l}\text { Dedicated } \\
\text { resources and } \\
\text { time }\end{array}$ \\
\hline & & & $\begin{array}{l}\text { Commitment to } \\
\text { Te Tiriti o } \\
\text { Waitangi }\end{array}$ & $\begin{array}{l}\text { Commitment to } \\
\text { Te Tiriti o } \\
\text { Waitangi }\end{array}$ \\
\hline & & & $\begin{array}{l}\text { Understanding of } \\
\text { equity }\end{array}$ & $\begin{array}{l}\text { Understanding of } \\
\text { equity }\end{array}$ \\
\hline
\end{tabular}

Table 9.15: Consolidation of key elements 


\subsection{THE SELF-ASSESSMENT MATURITY MATRIX}

This chapter presents the self-assessment maturity matrix that describes the key elements and outlines the maturity scale for these elements. The maturity matrix was developed with the senior clinical and operational leaders working in the New Zealand health system at the two workshops. The chapter outlines how the maturity matrix was developed, tested and refined (Figure 10.1) and presents what was found when successive versions were tested with three Alliance leadership teams (ALTs). These ALTs were selected based on the size of the population the DHB was serving and my prior knowledge of the ALT. The prior knowledge was based on my experience working as the SLM programme lead and included factors such as the membership of the ALT, how long they have existed, level of personnel changes in the ALT, quality of the SLM plans they have submitted, and improvement culture and capability. The chapter also summarises feedback received on the maturity matrix from the Māori Health Strategy and Policy team at the $\mathrm{MoH}$.

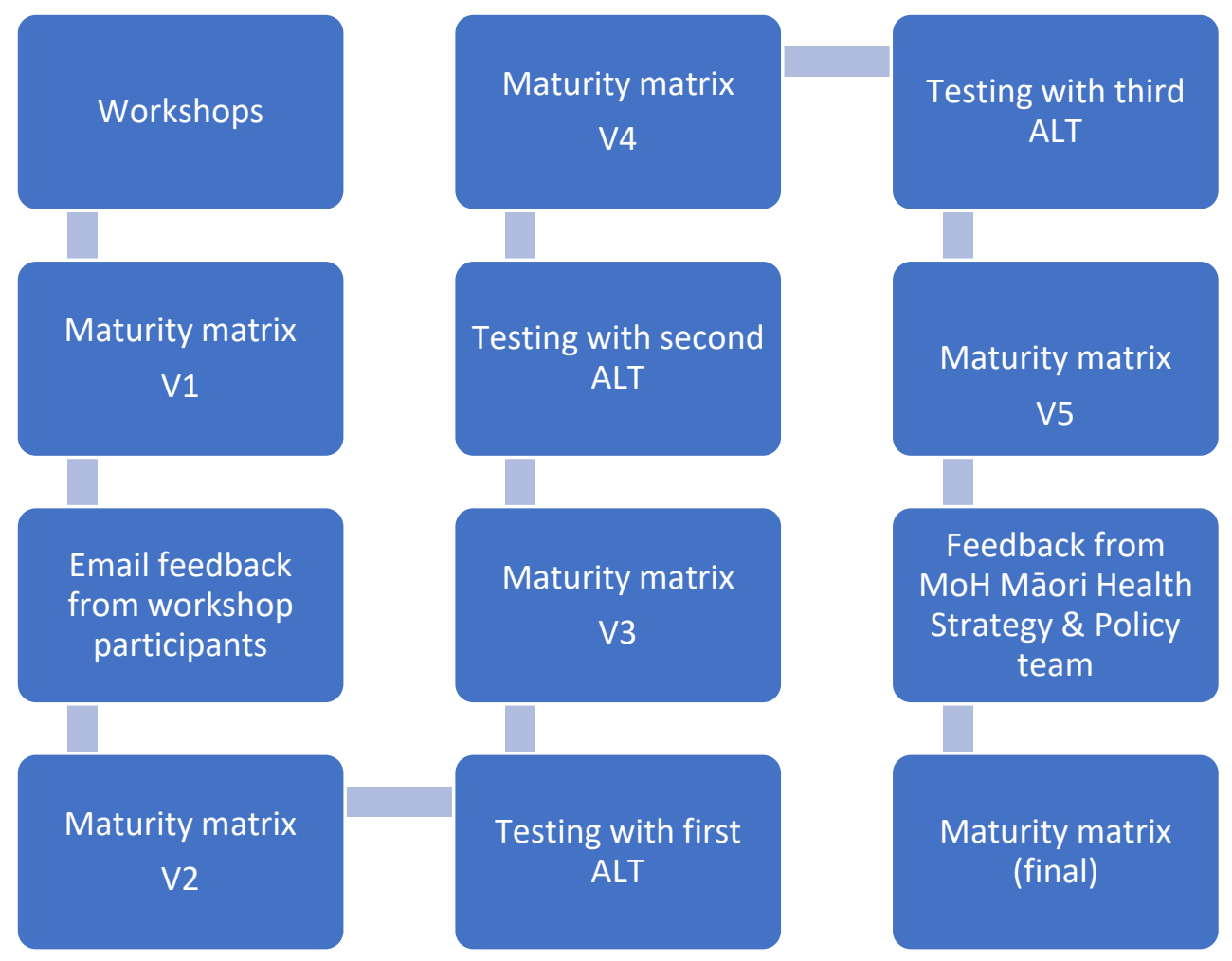

Figure 10.1: Iterative process for refining the maturity matrix 


\subsection{Purpose of the maturity matrix}

The aim was to develop a nationally consistent tool, with the knowledge of those working in the health system, to describe the key elements that support successful implementation of LST initiatives.

The research literature suggests that Alliances could use the maturity matrix to understand what the elements mean and what maturity for each element looks like; assess where they are on the scale for each element; and identify areas for development. Through a regular self-assessment process, the Alliances create feedback loops, which supports the notion of a learning system.

\subsection{Principles of the maturity matrix}

The maturity matrix was designed using the 10 key elements (identified in chapter 9) that support successful implementation of LST initiatives in the New Zealand health system. Except for two participants, others involved in the construction of the maturity matrix were same as those who attended the first workshop. The two additional participants did not attend the first workshop: one because of health reasons and the other participant's consent to participate in the workshop from their Chief Executive was delayed.

From my experience with the implementation of the SLM programme and evidence in literature from organisational behaviour, evolution and learning [70,161,162,194], I proposed to the workshop participants that the maturity matrix was underpinned with the improvement philosophy of constant learning and progressing positively along the maturity scale rather than achieving perfection. Workshop participants agreed with my proposal and emphasised that it was critical to demonstrate a commitment to Te Tiriti o Waitangi and achieving equity in the health system. The maturity matrix was constructed using complex system ideas: that it be simple and practical to use and specific enough to enable self-assessment but generic enough for local adaptability taking into consideration local context. The maturity matrix was designed for Alliances to use for assessing and enhancing their capacity and capability for successful implementation of LST initiatives through constant feedback loops.

\subsection{Development of the maturity matrix}

The first version of the maturity matrix was developed with participants in the second workshop (Table 10.1). Following the iterative process described in Figure 10.1, the maturity matrix underwent many revisions. 


\begin{tabular}{|c|c|c|c|c|c|}
\hline \multirow{2}{*}{$\begin{array}{l}\text { Key element and } \\
\text { Outcome descriptor } \\
\text { (what does the } \\
\text { established element } \\
\text { demonstrate?) }\end{array}$} & \multirow{2}{*}{$\begin{array}{l}\text { Indicators (What will show } \\
\text { this?) }\end{array}$} & \multicolumn{4}{|c|}{ Maturity scale - what we expect to see at each stage } \\
\hline & & Beginning (nothing in place) & $\begin{array}{l}\text { Emerging (something in } \\
\text { place) }\end{array}$ & $\begin{array}{l}\text { Established (this is what } \\
\text { good looks like) }\end{array}$ & $\begin{array}{l}\text { Excellence (outstanding e.g. } \\
\text { health and social } \\
\text { integration) }\end{array}$ \\
\hline \multirow{3}{*}{$\begin{array}{l}\text { Key element - Alliancing } \\
\text { way of working } \\
\text { Trust, shared sense of } \\
\text { purpose and } \\
\text { sustainability }\end{array}$} & $\begin{array}{l}\text { Structurally embedded in } \\
\text { the system (accountable } \\
\text { and visible) }\end{array}$ & $\begin{array}{l}\text { Initial discussion on shared } \\
\text { purpose }\end{array}$ & $\begin{array}{l}\text { Shared purpose agreed } \\
\text { Roles, responsibilities, } \\
\text { and accountability } \\
\text { understood }\end{array}$ & $\begin{array}{l}\text { Structurally embedded in } \\
\text { the system with clear } \\
\text { accountability and } \\
\text { appropriate resourcing }\end{array}$ & $\begin{array}{l}\text { Evolution over time } \\
\text { Expansion of partnership } \\
\text { into social sector }\end{array}$ \\
\hline & $\begin{array}{l}\text { The manner in which all } \\
\text { priority populations have } \\
\text { input into decision making }\end{array}$ & $\begin{array}{l}\text { Little or no evidence of } \\
\text { input } \\
\text { Tokenism or lip-service }\end{array}$ & $\begin{array}{l}\text { Mechanisms in place } \\
\text { Some populations have } \\
\text { influenced decision- } \\
\text { making }\end{array}$ & $\begin{array}{l}\text { System for influence } \\
\text { mature } \\
\text { Multiple populations have } \\
\text { influenced decision- } \\
\text { making via different } \\
\text { mechanisms } \\
\text { Evidence of innovation }\end{array}$ & $\begin{array}{l}\text { As in established and } \\
\text { widespread dissemination }\end{array}$ \\
\hline & $\begin{array}{l}\text { Depth of commitment to } \\
\text { partnership with Māori in all } \\
\text { aspects of alliance activity }\end{array}$ & $\begin{array}{l}\text { Māori representation (not } \\
\text { partnership) in alliance }\end{array}$ & $\begin{array}{l}\text { Key Māori leadership } \\
\text { and partnership at the } \\
\text { alliance } \\
\text { Alliance charter } \\
\text { recognises role of Treaty }\end{array}$ & $\begin{array}{l}\text { Treaty principles } \\
\text { embedded in the } \\
\text { philosophy and operation } \\
\text { of the alliance }\end{array}$ & $\begin{array}{l}\text { Full commitment to whānau } \\
\text { ora approach with multi- } \\
\text { sectorial and -dimensional } \\
\text { (holistic), integrative } \\
\text { systems and processes, } \\
\text { allow opportunities to } \\
\text { respond to community } \\
\text { aspirations }\end{array}$ \\
\hline
\end{tabular}




\begin{tabular}{|c|c|c|c|c|c|}
\hline \multirow{2}{*}{$\begin{array}{l}\text { Key element and } \\
\text { Outcome descriptor } \\
\text { (what does the } \\
\text { established element } \\
\text { demonstrate?) }\end{array}$} & \multirow{2}{*}{$\begin{array}{l}\text { Indicators (What will show } \\
\text { this?) }\end{array}$} & \multicolumn{4}{|c|}{ Maturity scale - what we expect to see at each stage } \\
\hline & & Beginning (nothing in place) & $\begin{array}{l}\text { Emerging (something in } \\
\text { place) }\end{array}$ & $\begin{array}{l}\text { Established (this is what } \\
\text { good looks like) }\end{array}$ & $\begin{array}{l}\text { Excellence (outstanding e.g. } \\
\text { health and social } \\
\text { integration) }\end{array}$ \\
\hline \multirow[t]{3}{*}{$\begin{array}{l}\text { Key element - Clinical } \\
\text { leadership and } \\
\text { engagement } \\
\text { Balanced influence } \\
\text { between management } \\
\text { and clinicians }\end{array}$} & $\begin{array}{l}\text { Managerial and clinical } \\
\text { leadership work as one }\end{array}$ & $\begin{array}{l}\text { Rebalancing of managerial } \\
\text { and clinical input into } \\
\text { system change development }\end{array}$ & $\begin{array}{l}\text { Trust model in place } \\
\text { between managerial and } \\
\text { clinical input } \\
\text { Trust and understanding } \\
\text { of the different roles }\end{array}$ & $\begin{array}{l}\text { Clinicians involved in } \\
\text { investment decisions } \\
\text { High attendance of clinical } \\
\text { leaders to alliance } \\
\text { meetings } \\
\text { Explicit sign off by } \\
\text { clinicians }\end{array}$ & $\begin{array}{l}\text { Distributed leadership and } \\
\text { shared sense of purpose } \\
\text { through honest } \\
\text { conversations, respect, } \\
\text { acknowledgement of roles, } \\
\text { and sustainable ongoing } \\
\text { leadership }\end{array}$ \\
\hline & $\begin{array}{l}\text { The degree to which } \\
\text { clinicians and managers } \\
\text { knowledge of population } \\
\text { health data is understood } \\
\text { and actioned }\end{array}$ & $\begin{array}{l}\text { Little or no evidence of } \\
\text { population health data } \\
\text { being considered by } \\
\text { managers and clinicians }\end{array}$ & $\begin{array}{l}\text { Some evidence of } \\
\text { meaningful discussion } \\
\text { between clinicians and } \\
\text { managers about equity } \\
\text { issues }\end{array}$ & $\begin{array}{l}\text { Work programme has } \\
\text { alignment between } \\
\text { population health data } \\
\text { and funding allocation } \\
\text { Clear evidence of regular } \\
\text { consideration of equity } \\
\text { issues between clinicians } \\
\text { and managers }\end{array}$ & $\begin{array}{l}\text { As in established and clear } \\
\text { improved outcomes and } \\
\text { national leadership }\end{array}$ \\
\hline & $\begin{array}{l}\text { Membership and way of } \\
\text { working reflects Treaty } \\
\text { principles }\end{array}$ & $\begin{array}{l}\text { Little or no Māori clinical } \\
\text { leadership }\end{array}$ & $\begin{array}{l}\text { Māori clinicians and } \\
\text { managers part of clinical } \\
\text { and alliance forums }\end{array}$ & $\begin{array}{l}\text { Evidence of Māori clinical } \\
\text { participation and influence } \\
\text { in investment decisions } \\
\text { High attendance of Māori } \\
\text { clinical leaders to alliance } \\
\text { meetings }\end{array}$ & $\begin{array}{l}\text { Dedicated workstream for } \\
\text { Māori outcomes } \\
\text { Multi-disciplinary and - } \\
\text { sectorial approach }\end{array}$ \\
\hline
\end{tabular}




\begin{tabular}{|c|c|c|c|c|c|}
\hline \multirow{2}{*}{$\begin{array}{l}\text { Key element and } \\
\text { Outcome descriptor } \\
\text { (what does the } \\
\text { established element } \\
\text { demonstrate?) }\end{array}$} & \multirow{2}{*}{$\begin{array}{l}\text { Indicators (What will show } \\
\text { this?) }\end{array}$} & \multicolumn{4}{|c|}{ Maturity scale - what we expect to see at each stage } \\
\hline & & Beginning (nothing in place) & $\begin{array}{l}\text { Emerging (something in } \\
\text { place) }\end{array}$ & $\begin{array}{l}\text { Established (this is what } \\
\text { good looks like) }\end{array}$ & $\begin{array}{l}\text { Excellence (outstanding e.g. } \\
\text { health and social } \\
\text { integration) }\end{array}$ \\
\hline \multirow[t]{3}{*}{$\begin{array}{l}\text { Key element - } \\
\text { Intelligent } \\
\text { commissioning } \\
\text { Intelligent planning } \\
\text { using evidence and data } \\
\text { to allocate resources }\end{array}$} & $\begin{array}{l}\text { Clear concise data for each } \\
\text { alliance decision }\end{array}$ & $\begin{array}{l}\text { Collection of data (linear) } \\
\text { Recognise need for } \\
\text { evidence-based decision- } \\
\text { making }\end{array}$ & $\begin{array}{l}\text { Build multiple data sets } \\
\text { Establishing data sharing } \\
\text { agreements across the } \\
\text { system }\end{array}$ & $\begin{array}{l}\text { Data tracked overtime to } \\
\text { show improvement } \\
\text { Data governance in place } \\
\text { to manage collection, } \\
\text { storage and release of } \\
\text { information }\end{array}$ & $\begin{array}{l}\text { Evidence-based decision- } \\
\text { making is transparent across } \\
\text { the system } \\
\text { Data governance including } \\
\text { Māori data sovereignty } \\
\text { recognised } \\
\text { Understanding of explicit } \\
\text { evidence-based investment } \\
\text { in Māori health }\end{array}$ \\
\hline & $\begin{array}{l}\text { Degree to which integrated } \\
\text { information and intelligence } \\
\text { for populations of interest } \\
\text { are explicitly considered and } \\
\text { managed routinely }\end{array}$ & $\begin{array}{l}\text { Information not accountable } \\
\text { Decisions ignore available } \\
\text { intelligence } \\
\text { No consideration of priority } \\
\text { population }\end{array}$ & $\begin{array}{l}\text { Some use for intelligence } \\
\text { that includes literature } \\
\text { review, simple analysis } \\
\text { of single data sets and } \\
\text { access to timely } \\
\text { information }\end{array}$ & $\begin{array}{l}\text { Timely, useful, thoughtful } \\
\text { intelligence available } \\
\text { Evidence of equity data } \\
\text { being considered in } \\
\text { decision-making }\end{array}$ & $\begin{array}{l}\text { All of the information } \\
\text { leading to targeted equity } \\
\text { funding } \\
\text { Exemplar for others } \\
\text { Contributing to } \\
\text { development of evidence } \\
\text { base }\end{array}$ \\
\hline & $\begin{array}{l}\text { Acknowledging resources to } \\
\text { Māori will be different }\end{array}$ & $\begin{array}{l}\text { Identifying the needs of } \\
\text { Māori }\end{array}$ & $\begin{array}{l}\text { Identifying resources for } \\
\text { Māori and allocating } \\
\text { appropriately }\end{array}$ & $\begin{array}{l}\text { De-invest in other areas } \\
\text { and re-invest into Māori } \\
\text { Prioritise new funding to } \\
\text { Māori }\end{array}$ & $\begin{array}{l}\text { Commissioning approaches } \\
\text { based on kaupapa Māori } \\
\text { models, for example } \\
\text { whānau ora }\end{array}$ \\
\hline
\end{tabular}


Key element and

Outcome descriptor

(what does the

established element

demonstrate?)

\section{Key element -}

Integrated health

information

Readily accessible

health information

which is responsive to

need
Indicators (What will show this?)

$\mathrm{NHI}$ linked data available to meet decision-making needs for the problem at hand

Degree to which integrated

information and intelligence

for populations of interest

are explicitly considered and

managed routinely

Māori have sovereignty over

integrated health

information
Maturity scale - what we expect to see at each stage

Beginning (nothing in place)

Emerging (something in place)

Agreement on the need for

data

Discussion on how to gather

Build multiple data sets

Establishing data sharing

agreements across the

system

Information not accountable

Decisions ignore available

intelligence

No consideration of priority population

Some use for intelligence

that includes literature

review, simple analysis

of single data sets and access to timely

information

Collection of broad data e.g. Māori data sovereignty

national, patients identifying recognised on data as Māori

sharing agreements
Established (this is what good looks like)

Current data from multiple data sources which is accessible, equity focused and consistently linked via $\mathrm{NHI}$

Timely, useful, thoughtful

intelligence available

Evidence of equity data

being considered in

decision-making

Established data collection systems that links Māori data via NHI
Excellence (outstanding e.g.

health and social

integration)

The right information at the

right time for forecasting

and feedback to drive

continuous improvement

All the information leading

to targeted equity funding

Exemplar for others

Contributing to

development of evidence

base

New knowledge can inform

traditional model of care

Evolution of NZ healthcare 


\begin{tabular}{|c|c|c|c|c|c|}
\hline \multirow{2}{*}{$\begin{array}{l}\text { Key element and } \\
\text { Outcome descriptor } \\
\text { (what does the } \\
\text { established element } \\
\text { demonstrate?) }\end{array}$} & \multirow{2}{*}{$\begin{array}{l}\text { Indicators (What will show } \\
\text { this?) }\end{array}$} & \multicolumn{4}{|c|}{ Maturity scale - what we expect to see at each stage } \\
\hline & & Beginning (nothing in place) & $\begin{array}{l}\text { Emerging (something in } \\
\text { place) }\end{array}$ & $\begin{array}{l}\text { Established (this is what } \\
\text { good looks like) }\end{array}$ & $\begin{array}{l}\text { Excellence (outstanding e.g. } \\
\text { health and social } \\
\text { integration) }\end{array}$ \\
\hline \multirow[t]{3}{*}{$\begin{array}{l}\text { Key element - Analytic } \\
\text { capability } \\
\text { Mindset, skillset, } \\
\text { knowledge available to } \\
\text { turn all intelligence } \\
\text { about health of local } \\
\text { populations into insights } \\
\text { about variation and } \\
\text { change in quality and } \\
\text { outcomes of healthcare }\end{array}$} & $\begin{array}{l}\text { Intelligence about health of } \\
\text { local populations into } \\
\text { insights about variation and } \\
\text { change in quality and } \\
\text { outcomes of healthcare }\end{array}$ & $\begin{array}{l}\text { Recognition that intelligence } \\
\text { is required } \\
\text { Initial discussion to establish } \\
\text { common tools, data } \\
\text { definition and analytic } \\
\text { approach }\end{array}$ & $\begin{array}{l}\text { Investment in tools and } \\
\text { resources } \\
\text { Emerging information } \\
\text { drives some decisions } \\
\text { Growing familiarity and } \\
\text { use of data to inform } \\
\text { decision }\end{array}$ & $\begin{array}{l}\text { Growing sophistication on } \\
\text { the level of data needed at } \\
\text { different decision points } \\
\text { Consensus on definition, } \\
\text { tools and approach } \\
\text { Delivery of consistent } \\
\text { analysis over time }\end{array}$ & $\begin{array}{l}\text { Use of analytics across } \\
\text { short, medium and long } \\
\text { term } \\
\text { Starts to align allowing CQI } \\
\text { loop to include feedback to } \\
\text { forward projection and } \\
\text { confidence } \\
\text { Cross district learning and } \\
\text { sharing }\end{array}$ \\
\hline & $\begin{array}{l}\text { Degree to which intelligence } \\
\text { resources are allocated to } \\
\text { analysing data with an } \\
\text { equity focus }\end{array}$ & $\begin{array}{l}\text { No capacity dedicated to } \\
\text { analysing equity } \\
\text { Little understanding of } \\
\text { analysing variation or } \\
\text { measuring change }\end{array}$ & $\begin{array}{l}\text { Limited dedicated } \\
\text { analytic capability and } \\
\text { capacity for equity } \\
\text { Limited understanding of } \\
\text { analysing variation or } \\
\text { intelligence }\end{array}$ & $\begin{array}{l}\text { Appropriate analytic } \\
\text { capacity and capability, } \\
\text { and intelligence to } \\
\text { influence the system } \\
\text { Collaborate with clinicians } \\
\text { to interrogate iteratively } \\
\text { and tell the story }\end{array}$ & $\begin{array}{l}\text { As above and able to } \\
\text { provide this service for high } \\
\text { priority populations }\end{array}$ \\
\hline & $\begin{array}{l}\text { Degree to which intelligence } \\
\text { resources are allocated to } \\
\text { analysing data for Māori in } \\
\text { partnership with local Māori }\end{array}$ & $\begin{array}{l}\text { Equity data acknowledged } \\
\text { without local specific } \\
\text { knowledge or partnership }\end{array}$ & $\begin{array}{l}\text { Data includes Māori } \\
\text { aspiration and reflects } \\
\text { Māori mindset }\end{array}$ & $\begin{array}{l}\text { Redefine the experience } \\
\text { from a framework of the } \\
\text { Treaty principles and data } \\
\text { therefore become } \\
\text { relevant for Māori }\end{array}$ & $\begin{array}{l}\text { Emergence of new } \\
\text { knowledge and evidence } \\
\text { that is holistic and } \\
\text { encompassing }\end{array}$ \\
\hline
\end{tabular}




\begin{tabular}{|c|c|c|c|c|c|}
\hline \multirow{2}{*}{$\begin{array}{l}\text { Key element and } \\
\text { Outcome descriptor } \\
\text { (what does the } \\
\text { established element } \\
\text { demonstrate?) }\end{array}$} & \multirow{2}{*}{$\begin{array}{l}\text { Indicators (What will show } \\
\text { this?) }\end{array}$} & \multicolumn{4}{|c|}{ Maturity scale - what we expect to see at each stage } \\
\hline & & Beginning (nothing in place) & $\begin{array}{l}\text { Emerging (something in } \\
\text { place) }\end{array}$ & $\begin{array}{l}\text { Established (this is what } \\
\text { good looks like) }\end{array}$ & $\begin{array}{l}\text { Excellence (outstanding e.g. } \\
\text { health and social } \\
\text { integration) }\end{array}$ \\
\hline \multirow{3}{*}{$\begin{array}{l}\text { Key element - } \\
\text { Continuous } \\
\text { improvement } \\
\text { Mindset, skills, } \\
\text { knowledge and } \\
\text { experience of using } \\
\text { quality improvement } \\
\text { science and techniques } \\
\text { to drive measurable } \\
\text { impact in quality and } \\
\text { outcomes of the health } \\
\text { system }\end{array}$} & $\begin{array}{l}\text { Specific resource and } \\
\text { funding put aside for a } \\
\text { continuous improvement } \\
\text { platform in alliancing }\end{array}$ & $\begin{array}{l}\text { Recognition that conscious } \\
\text { investment in CQI is } \\
\text { worthwhile } \\
\text { Initial planning to enable } \\
\text { this discussed }\end{array}$ & $\begin{array}{l}\text { Resourcing in CQI in } \\
\text { place }\end{array}$ & $\begin{array}{l}\text { CQI activities are part of } \\
\text { every decision and } \\
\text { implementation and } \\
\text { continued feedback loops } \\
\text { Established CQI } \\
\text { monitoring system }\end{array}$ & $\begin{array}{l}\text { CQI should lead to improved } \\
\text { systems outcomes overtime } \\
\text { in health and social sectors } \\
\text { Agreed outcomes across } \\
\text { health and social sectors }\end{array}$ \\
\hline & $\begin{array}{l}\text { Degree to which you can } \\
\text { demonstrate population } \\
\text { groupings and equity gap is } \\
\text { reducing as a result of CQI } \\
\text { activity undertaken (no } \\
\text { minimum targets) }\end{array}$ & $\begin{array}{l}\text { CQI activities have had no } \\
\text { effect on inequities or } \\
\text { worsened }\end{array}$ & $\begin{array}{l}\text { Existing or new CQI } \\
\text { activities consider } \\
\text { inequity but effects are } \\
\text { unclear or inconsistent }\end{array}$ & $\begin{array}{l}\text { Existing and new CQI } \\
\text { activities consistently } \\
\text { associated with } \\
\text { improvements in equity }\end{array}$ & $\begin{array}{l}\text { CQI activities lead to } \\
\text { continuous and significant } \\
\text { reductions in inequities } \\
\text { across multiple priority } \\
\text { populations }\end{array}$ \\
\hline & $\begin{array}{l}\text { Māori aspirations are } \\
\text { integrated at stage of } \\
\text { improvement journey } \\
\text { Māori knowledge is } \\
\text { respected }\end{array}$ & $\begin{array}{l}\text { Awareness traditional CQI } \\
\text { platform is not always } \\
\text { appropriate for Māori }\end{array}$ & $\begin{array}{l}\text { Identification of Māori } \\
\text { improvement models } \\
\text { e.g. whare tapa wha } \\
\text { model }\end{array}$ & $\begin{array}{l}\text { Embedded Māori } \\
\text { improvement models }\end{array}$ & $\begin{array}{l}\text { Embedded Māori } \\
\text { improvement models with } \\
\text { social sectors }\end{array}$ \\
\hline
\end{tabular}




\begin{tabular}{|c|c|c|c|c|c|}
\hline \multirow{2}{*}{$\begin{array}{l}\text { Key element and } \\
\text { Outcome descriptor } \\
\text { (what does the } \\
\text { established element } \\
\text { demonstrate?) }\end{array}$} & \multirow{2}{*}{$\begin{array}{l}\text { Indicators (What will show } \\
\text { this?) }\end{array}$} & \multicolumn{4}{|c|}{ Maturity scale - what we expect to see at each stage } \\
\hline & & Beginning (nothing in place) & $\begin{array}{l}\text { Emerging (something in } \\
\text { place) }\end{array}$ & $\begin{array}{l}\text { Established (this is what } \\
\text { good looks like) }\end{array}$ & $\begin{array}{l}\text { Excellence (outstanding e.g. } \\
\text { health and social } \\
\text { integration) }\end{array}$ \\
\hline \multirow{3}{*}{$\begin{array}{l}\text { Key element - } \\
\text { Dedicated resources and } \\
\text { time } \\
\text { Sustainable resources } \\
\text { located in the right } \\
\text { place in the system for } \\
\text { decision-making }\end{array}$} & $\begin{array}{l}\text { Resources ringfenced to } \\
\text { manage and support change } \\
\text { management and decision- } \\
\text { making }\end{array}$ & $\begin{array}{l}\text { Alliancing is seen as a good } \\
\text { idea but embryonic form, } \\
\text { function and dedicated } \\
\text { resources } \\
\text { Relies on goodwill }\end{array}$ & $\begin{array}{l}\text { Realisation alliancing } \\
\text { needs to be resourced } \\
\text { within the system } \\
\text { Regular alliance } \\
\text { meetings funded and } \\
\text { some secretariat support }\end{array}$ & $\begin{array}{l}\text { Dedicated management } \\
\text { and clinical resource for } \\
\text { alliancing to occur } \\
\text { Independent programme } \\
\text { office }\end{array}$ & $\begin{array}{l}\text { Resource is embedded in } \\
\text { the system with a long-term } \\
\text { view of planning } \\
\text { Ongoing sustainable } \\
\text { resourcing for alliancing in } \\
\text { the system }\end{array}$ \\
\hline & $\begin{array}{l}\text { Degree to which equity is } \\
\text { explicitly incorporated into } \\
\text { all elements of alliance work } \\
\text { programme }\end{array}$ & $\begin{array}{l}\text { Equity mentioned in alliance } \\
\text { terms of reference but no } \\
\text { further evidence or funding }\end{array}$ & $\begin{array}{l}\text { Mention of equity in } \\
\text { alliance related work } \\
\text { At least some funded } \\
\text { initiatives especially } \\
\text { aimed at improving } \\
\text { equity }\end{array}$ & $\begin{array}{l}\text { Multiple funded equity } \\
\text { interventions aimed at } \\
\text { several priority } \\
\text { populations } \\
\text { Some successful outcomes } \\
\text { in reducing inequities }\end{array}$ & $\begin{array}{l}\text { As in established with } \\
\text { multiple successful funded } \\
\text { initiatives }\end{array}$ \\
\hline & $\begin{array}{l}\text { Resources are prioritised } \\
\text { around the Treaty } \\
\text { commitments } \\
\text { Core business spending is } \\
\text { challenged }\end{array}$ & $\begin{array}{l}\text { Initial dialogue with local iwi } \\
\text { to inform resource } \\
\text { allocation and funding }\end{array}$ & $\begin{array}{l}\text { Dedicated resources for } \\
\text { Māori health outcomes }\end{array}$ & $\begin{array}{l}\text { Equity based decision- } \\
\text { making on funding and } \\
\text { resource allocations }\end{array}$ & $\begin{array}{l}\text { As in established with multi- } \\
\text { sectorial }\end{array}$ \\
\hline
\end{tabular}




\begin{tabular}{|c|c|c|c|c|c|}
\hline \multirow{2}{*}{$\begin{array}{l}\text { Key element and } \\
\text { Outcome descriptor } \\
\text { (what does the } \\
\text { established element } \\
\text { demonstrate?) }\end{array}$} & \multirow{2}{*}{$\begin{array}{l}\text { Indicators (What will show } \\
\text { this?) }\end{array}$} & \multicolumn{4}{|c|}{ Maturity scale - what we expect to see at each stage } \\
\hline & & Beginning (nothing in place) & $\begin{array}{l}\text { Emerging (something in } \\
\text { place) }\end{array}$ & $\begin{array}{l}\text { Established (this is what } \\
\text { good looks like) }\end{array}$ & $\begin{array}{l}\text { Excellence (outstanding e.g. } \\
\text { health and social } \\
\text { integration) }\end{array}$ \\
\hline \multirow[t]{3}{*}{$\begin{array}{l}\text { Key element - } \\
\text { Collaborative approach } \\
\text { Teams and professions } \\
\text { working together }\end{array}$} & $\begin{array}{l}\text { Agreement on a transparent } \\
\text { decision-making process } \\
\text { that the system enables }\end{array}$ & $\begin{array}{l}\text { Setting up an alliance } \\
\text { agreement }\end{array}$ & $\begin{array}{l}\text { Identifying the } \\
\text { organisational } \\
\text { participants, clinical, } \\
\text { managerial and other } \\
\text { (Māori and Pacific) as } \\
\text { part of the alliance }\end{array}$ & $\begin{array}{l}\text { Active participation and } \\
\text { collaboration from all } \\
\text { participants } \\
\text { Includes a governance } \\
\text { structure } \\
\text { Inclusion of broader } \\
\text { health sector }\end{array}$ & $\begin{array}{l}\text { Active participation and } \\
\text { collaboration across all } \\
\text { sectors }\end{array}$ \\
\hline & $\begin{array}{l}\text { The degree to which } \\
\text { organisations with a } \\
\text { concern for priority } \\
\text { populations are included in } \\
\text { integrated programme } \\
\text { (design, implement and } \\
\text { evaluate) }\end{array}$ & $\begin{array}{l}\text { Little if any involvement of } \\
\text { appropriate organisations } \\
\text { (only DHBs and PHOs } \\
\text { involved) }\end{array}$ & $\begin{array}{l}\text { Beginnings of integration } \\
\text { within health alone } \\
\text { including relevant } \\
\text { priority population } \\
\text { groups }\end{array}$ & $\begin{array}{l}\text { Widespread integration } \\
\text { with relevant health and } \\
\text { disability NGOs with } \\
\text { priority population } \\
\text { interest } \\
\text { Beginnings of de-siloed } \\
\text { funding }\end{array}$ & $\begin{array}{l}\text { Collaboration within and } \\
\text { beyond health to } \\
\text { appropriate priority } \\
\text { population groups including } \\
\text { design, implementation and } \\
\text { evaluation of programmes } \\
\text { Mature de-siloed funding }\end{array}$ \\
\hline & $\begin{array}{l}\text { Acknowledging Māori as the } \\
\text { Treaty partner }\end{array}$ & $\begin{array}{l}\text { Little or no engagement } \\
\text { with Māori providers }\end{array}$ & $\begin{array}{l}\text { Māori providers are } \\
\text { involved in the decision- } \\
\text { making process }\end{array}$ & $\begin{array}{l}\text { Established Māori partners } \\
\text { (Iwi, hapū, providers etc) } \\
\text { actively involved in the } \\
\text { decision-making process }\end{array}$ & $\begin{array}{l}\text { Māori are leading, chairing } \\
\text { the decision-making process } \\
\text { in the alliance leadership } \\
\text { teams }\end{array}$ \\
\hline
\end{tabular}




\begin{tabular}{|c|c|c|c|c|c|}
\hline \multirow{2}{*}{$\begin{array}{l}\text { Key element and } \\
\text { Outcome descriptor } \\
\text { (what does the } \\
\text { established element } \\
\text { demonstrate?) }\end{array}$} & \multirow{2}{*}{$\begin{array}{l}\text { Indicators (What will show } \\
\text { this?) }\end{array}$} & \multicolumn{4}{|c|}{ Maturity scale - what we expect to see at each stage } \\
\hline & & Beginning (nothing in place) & $\begin{array}{l}\text { Emerging (something in } \\
\text { place) }\end{array}$ & $\begin{array}{l}\text { Established (this is what } \\
\text { good looks like) }\end{array}$ & $\begin{array}{l}\text { Excellence (outstanding e.g. } \\
\text { health and social } \\
\text { integration) }\end{array}$ \\
\hline \multirow[t]{3}{*}{$\begin{array}{l}\text { Key element - Equity } \\
\text { Widespread } \\
\text { dissemination and } \\
\text { understanding }\end{array}$} & $\begin{array}{l}\text { Every decision has an equity } \\
\text { focus based on coherent } \\
\text { information }\end{array}$ & $\begin{array}{l}\text { Equity mentioned but not } \\
\text { yet widely understood or } \\
\text { explicitly addressed }\end{array}$ & $\begin{array}{l}\text { Start to see equity as key } \\
\text { part of regular } \\
\text { conversation including } \\
\text { discussion on how to } \\
\text { address inequities }\end{array}$ & $\begin{array}{l}\text { Explicit influencing work } \\
\text { programmes and } \\
\text { investments } \\
\text { Monitoring of } \\
\text { effectiveness of change to } \\
\text { close gaps } \\
\text { Services designed with } \\
\text { service users }\end{array}$ & $\begin{array}{l}\text { Health and social sector } \\
\text { partner collaboratively to } \\
\text { reduce inequities including a } \\
\text { focus on determinants of } \\
\text { health }\end{array}$ \\
\hline & $\begin{array}{l}\text { Degree to which equity is } \\
\text { explicit in inputs and } \\
\text { outputs and is explained } \\
\text { front and centre }\end{array}$ & $\begin{array}{l}\text { Equity mentioned in alliance } \\
\text { terms of reference }\end{array}$ & $\begin{array}{l}\text { Equity particularly } \\
\text { mentioned in both } \\
\text { internal documentation } \\
\text { and reports with } \\
\text { attempts to present } \\
\text { equity data }\end{array}$ & $\begin{array}{l}\text { Equity explained in all } \\
\text { documentations } \\
\text { Evidence of equity } \\
\text { commentary in all reports } \\
\text { Evidence of deliberate } \\
\text { interpretive and action } \\
\text { reporting }\end{array}$ & $\begin{array}{l}\text { Evidence of public } \\
\text { awareness of equity through } \\
\text { alliance activity }\end{array}$ \\
\hline & $\begin{array}{l}\text { Māori health and wellness } \\
\text { are the priority focus }\end{array}$ & Cultural training provided & $\begin{array}{l}\text { More integrated and not } \\
\text { dependent on Māori } \\
\text { leadership to provide } \\
\text { equity lens }\end{array}$ & $\begin{array}{l}\text { Entire leadership team } \\
\text { actively demonstrate } \\
\text { behaviours in policy, } \\
\text { practice and decision- } \\
\text { making }\end{array}$ & $\begin{array}{l}\text { Greater inter-sectorial } \\
\text { integration across alliance } \\
\text { framework }\end{array}$ \\
\hline
\end{tabular}


Key element and

Outcome descriptor

(what does the

established element

demonstrate?)

\section{Key element -}

Commitment to Te Tiriti

o Waitangi

Acknowledgement,

awareness, removing of

institutional racism
Indicators (What will show this?)

Decision-making processes acknowledge and corrects for the impact and role of institutional racism

Common understanding of the impact of the Treaty on the alliance and its decisionmaking processes

Degree to which the issue of biculturalism vs the wider priority population is understood

Acknowledging Māori as a Treaty partner
Maturity scale - what we expect to see at each stage

\begin{tabular}{|l|l|}
\hline Beginning (nothing in place) & $\begin{array}{l}\text { Emerging (something in } \\
\text { place) }\end{array}$ \\
\hline $\begin{array}{l}\text { Early recognition of Treaty } \\
\text { responsibilities }\end{array}$ & $\begin{array}{l}\text { Explicit } \\
\text { acknowledgement of } \\
\text { Treaty responsibilities } \\
\text { and proactive steps to } \\
\text { engage in particular to } \\
\text { lower institutional bias }\end{array}$ \\
\hline $\begin{array}{l}\text { Confusion between the } \\
\text { Treaty and equity }\end{array}$ & $\begin{array}{l}\text { Evidence that the } \\
\text { delineation between } \\
\text { both is acknowledged } \\
\text { and documented }\end{array}$ \\
\hline $\begin{array}{l}\text { Basic information on the } \\
\text { Treaty, racism and bias } \\
\text { provided to alliance and } \\
\text { organisations }\end{array}$ & $\begin{array}{l}\text { Understanding the } \\
\text { Treaty obligations by } \\
\text { alliance and } \\
\text { organisations }\end{array}$ \\
\hline
\end{tabular}

Established (this is what good looks like)

\section{health and social}

integration)

Established Treaty

Active partnership is

partnership between Iwi, demonstrating improved

hapū and alliance focussed outcomes

on reducing institutional

bias and improving

outcomes

Evidence that special

obligation of Treaty results

in documented actions

leading to improved outcomes for Māori

Treaty workshops

including racism and

discrimination established

within the institution
Awareness used as exemplar for others

Evolving actions on the Treaty, racism and bias reviewed and monitored on regular basis 


\begin{tabular}{|c|c|c|c|c|c|}
\hline \multirow{2}{*}{$\begin{array}{l}\text { Key element and } \\
\text { Outcome descriptor } \\
\text { (what does the } \\
\text { established element } \\
\text { demonstrate?) }\end{array}$} & \multirow{2}{*}{$\begin{array}{l}\text { Indicators (What will show } \\
\text { this?) }\end{array}$} & \multicolumn{4}{|c|}{ Maturity scale - what we expect to see at each stage } \\
\hline & & Beginning (nothing in place) & $\begin{array}{l}\text { Emerging (something in } \\
\text { place) }\end{array}$ & $\begin{array}{l}\text { Established (this is what } \\
\text { good looks like) }\end{array}$ & $\begin{array}{l}\text { Excellence (outstanding e.g. } \\
\text { health and social } \\
\text { integration) }\end{array}$ \\
\hline \multirow[t]{3}{*}{$\begin{array}{l}\text { Key element - Engaged } \\
\text { people, whanau and } \\
\text { community } \\
\text { Pathways of } \\
\text { engagement that } \\
\text { acknowledge agenda } \\
\text { and focus }\end{array}$} & $\begin{array}{l}\text { Information is available to } \\
\text { communities to understand } \\
\text { what is important to them }\end{array}$ & $\begin{array}{l}\text { Acknowledge the need to } \\
\text { have the right people } \\
\text { around the table }\end{array}$ & $\begin{array}{l}\text { Acknowledgement that } \\
\text { engagement is broader } \\
\text { than a seat at the table } \\
\text { That it is how the } \\
\text { alliance engagement } \\
\text { with its broader } \\
\text { community to prioritise } \\
\text { and exchange } \\
\text { information }\end{array}$ & $\begin{array}{l}\text { A multi-channel } \\
\text { engagement plan in place } \\
\text { Meaningful input from } \\
\text { service users and } \\
\text { communities leading to } \\
\text { changed behaviours }\end{array}$ & $\begin{array}{l}\text { Communities believe that } \\
\text { the right decisions are being } \\
\text { made for services they need } \\
\text { Community is engaged in } \\
\text { decision-making for re- } \\
\text { prioritising to changing need }\end{array}$ \\
\hline & $\begin{array}{l}\text { Degree to which individual, } \\
\text { whānau and community } \\
\text { voice from all populations is } \\
\text { heard and acted upon in } \\
\text { decision-making }\end{array}$ & $\begin{array}{l}\text { At least one service user } \\
\text { from high priority } \\
\text { populations paid to attend } \\
\text { alliance meetings }\end{array}$ & $\begin{array}{l}\text { Service user insight from } \\
\text { priority populations } \\
\text { sought across all } \\
\text { activities of the alliance }\end{array}$ & $\begin{array}{l}\text { Evidence of service user } \\
\text { input in all aspects of } \\
\text { activities } \\
\text { Evidence that input is } \\
\text { influential } \\
\text { Service users change } \\
\text { service workstream }\end{array}$ & $\begin{array}{l}\text { Individual, whānau and } \\
\text { community voice influence } \\
\text { decision-making across the } \\
\text { alliance }\end{array}$ \\
\hline & $\begin{array}{l}\text { Māori are partners in all } \\
\text { stages of design, delivery } \\
\text { and implementation }\end{array}$ & $\begin{array}{l}\text { Acknowledgement that } \\
\text { Māori need to be around } \\
\text { the table }\end{array}$ & $\begin{array}{l}\text { Terms of reference that } \\
\text { are mutually agreed with } \\
\text { Māori partners }\end{array}$ & $\begin{array}{l}\text { Iwi partnership in } \\
\text { decision-making }\end{array}$ & $\begin{array}{l}\text { Iwi partnership in multi- } \\
\text { sectorial decision-making }\end{array}$ \\
\hline
\end{tabular}


The maturity matrix was tested with three Alliance leadership teams (ALTs) to determine if:

- it was functioning as a learning tool

- $\quad$ it helped Alliances see where they were on the improvement journey

- it helped Alliances identify areas of improvement, such as broadening the ALT membership and ensuring they have a terms of reference that articulates the expected behaviours from their members

- if acted as a resource to create a stimulus for change

- $\quad$ it was easy and practical to use.

The maturity matrix was refined following each stage of the testing. Quotes from transcripts of audio-recorded group feedback sessions are used to highlight participants' feedback.

To help readers understand feedback from the testing process, the outline of the maturity matrix, as it evolved after the first testing, is shown in Table 10.2.

\begin{tabular}{|c|c|c|c|c|c|c|}
\hline \multicolumn{7}{|c|}{$\begin{array}{l}\text { Introduction to the self-assessment maturity } \mathrm{m} \\
\text { - How the maturity matrix was developed } \\
\text { - Purpose of the maturity matrix } \\
\text { - Principles of the maturity matrix } \\
\text { - } \quad \text { Purpose of testing }\end{array}$} \\
\hline \multicolumn{7}{|c|}{ Key elements and their descriptions } \\
\hline \multicolumn{7}{|c|}{ For each element e.g. Alliancing way of working } \\
\hline \multirow{2}{*}{$\begin{array}{l}\text { Outcome } \\
\text { descriptor }\end{array}$} & \multirow{2}{*}{$\begin{array}{l}\text { Indicators } \\
\text { (What will show } \\
\text { this?) }\end{array}$} & \multicolumn{4}{|c|}{ Maturity scale - what we expect to see at each stage } & \\
\hline & & $\begin{array}{l}\text { Beginning } \\
\quad=0\end{array}$ & $\begin{array}{l}\text { Emerging } \\
\quad=1\end{array}$ & $\begin{array}{c}\text { Established } \\
\quad=2\end{array}$ & $\begin{array}{l}\text { Excellence } \\
\quad=3\end{array}$ & Score \\
\hline \multirow{3}{*}{$\begin{array}{l}\text { What does } \\
\text { the } \\
\text { established } \\
\text { element } \\
\text { demonstrate? }\end{array}$} & $\begin{array}{l}\text { Whole-of- } \\
\text { system } \\
\text { indicator }\end{array}$ & & & & & \\
\hline & Equity indicator & & & & & \\
\hline & $\begin{array}{l}\text { Te Tiriti o } \\
\text { Waitangi } \\
\text { indicator }\end{array}$ & & & & & \\
\hline
\end{tabular}

Table 10.2: Outline of the maturity matrix following first testing

ALTs have varied membership depending on the make-up of the Alliances. Some Alliances have a broad membership that includes senior DHB and PHO managers and clinicians, consumer advocates, and representatives from community health services such as pharmacy, Māori and 
Pacific providers and district nursing (ALTs one and three). Other Alliances only have senior DHB and PHO managers and clinicians (ALT two).

\subsection{Feedback from the Alliance leadership teams on the maturity matrix}

\subsubsection{Alliance leadership team one}

The ALT agreed that the key elements provided adequate coverage of the areas considered important to the Alliance; the Alliance felt inspired to change to shift their maturity along the scale; and that the maturity matrix acted as a resource to create stimulus for change. Suggestions made to improve the maturity matrix are presented below. The amended version of the maturity matrix following the testing was provided to the ALT and no further feedback was received.

\section{Consistency on language used in the maturity matrix}

The ALT provided detailed feedback with handwritten notes on the hard copy of the maturity matrix to highlight language inconsistencies or suggestions to improve the language used. For example:

Be consistent with the language it says commitment to Te Tiriti o Waitangi and then it talks about the Treaty. Now, the understanding of both these things are different. The Treaty, we're talking about what people read in the English version. Te Tiriti o Waitangi is Māori version and they're actually interpreted differently.

\section{Maturity scale content}

The ALT provided feedback on some of the indicators and content of the maturity scale to clarify and strengthened, for example:

That indicator it says, 'Data protects mana, purpose of data collection is explained and shared with Maori'. We thought it a condescending statement in that it kind of says that Maori don't actually know what data is about.

Just on number six, you've got frontline managers. We thought about frontline staff rather than just limiting it to managers. You might have different levels of data within the different levels in an organisation, so data relevant to a frontline person might be different to a manager.

If we're talking about system transformation, then it's really not just about best health outcomes. It's actually something around the best wellbeing outcomes because it's health, social, justice etc, etc. Also, it's not around therefore just the 
development of the clinical pathways, there's other pathways that can enable intelligent commissioning. Like we would say Whānau Ora pathway isn't a clinical pathway but it's a pathway. And then I'd say pathways to service specification, then I put and 'management or procurement through commissioning' because we're still stuck in contract management or procurement. We need to be moving to commissioning frameworks.

And just that one that says acknowledging Māori as a Treaty partner. It's not Māori; Iwi are partner under the Treaty, not Māori. So that should be acknowledging Iwi as the Treaty partner.

\section{General feedback}

There was some confusion on who was being assessed; the DHB, PHO or the Alliance, therefore the ALT suggested inclusion of an introduction section outlining the purpose and principles of the maturity matrix, which would help clarify this. The ALT also suggested adding a row after each element to sum up the scores for the element.

\section{My observations}

On arrival I noticed the room was set up in a board room layout, with light snacks and drinks available for meeting attendees. As the attendees started arriving, by the social nature of the conversations, it was apparent that members of this ALT were familiar with each other and had a positive relationship. There were several outstanding consent forms, so the facilitator assisted in collection of the completed consent forms from all attendees. Participants were given option to leave the meeting if they didn't want to participate in the testing process.

The energy level in the room was positive and vibrant with lots of discussion occurring. The testing processed surpassed my expectations. There was positive energy in the room all through the duration of the testing. I felt welcomed by the group and was offered drink and snacks. The group was actively engaged, and forthcoming with their feedback in a constructive way. The chair and the group prioritised the testing process on their agenda and did not want to rush the process. They decided to delay some of the other agenda items to allow more time for this process. This reflected the willingness of the Alliance to participate in the testing process. It was evident that there was established and high trust relationship between the attendees and they were aware of their purpose and understood the value of continuous improvement. 


\subsubsection{Alliance leadership team two feedback}

The ALT agreed that the key elements provided adequate coverage of the areas considered important to the Alliance; the Alliance felt inspired to change to shift their maturity along the scale; and that the maturity matrix acted as a resource to create stimulus for change. Suggestions made to improve the maturity matrix are presented below. The ALT discussed how to fit this assessment as a regular process into an already busy agenda of the Alliance. Members recognised the importance of reviewing how the Alliance is working but found it challenging to prioritise a self-assessment process such as this in their agenda.

\section{Maturity scale content}

Suggestions to improve the maturity matrix were:

- To add narrative on the outcome descriptors so the story of improvement is clear, and the Alliance can clearly see why they would want to improve and how to shift along the scale. This might reduce the debate on the criteria.

- Clarify in the introduction page that it is the Alliance that is being assessed and not individual providers.

- Would like a larger score range, maybe from 1-12 to allow the Alliance to score accurately their place in the scale.

- A score of zero is not a good enabler of change as Alliances may hesitate to score themselves a zero, which may negatively affect their engagement in the self-assessment process. Starting from score of one was recommended as this will acknowledge the Alliance is on an improvement journey, albeit in the early stage of the maturity scale.

- More description on and importance for trust should be given to the alliancing way of working element as this is the most important tenet of this element.

- A page for the Alliance to prioritise their next steps following the self-assessment process in the form of an action plan.

\section{My observations}

Testing process with this ALT was a very different experience compared to the first ALT. The ALT chair was keen on testing the framework even when the ALT meeting was cancelled. Although the cancellation of the meeting reduced the number of participants, it did not have a significant impact as only seven out of 16 participants had confirmed their attendance prior to the meeting being cancelled. Two participants and the facilitator joining via zoom made the discussion a bit challenging. However, given the geographical boundary of this district, the ALT is used to this way 
of working. This added another complexity to the testing of the maturity matrix where all participants were not in a room together, as was the case for the first ALT. Another variable different for this ALT was that the participants were all health care professionals. Given the low number of participants, I did consider cancelling or rescheduling the testing. However, the lack of attendance also reflected the maturity and functionality of the ALT. Some of the DHB operational leaders couldn't attend because of another event. Participants who attended seemed to have good relationships with each other and understood the importance of continuous improvement culture as evidenced by their willingness to participate in the selfassessment process.

During the self-assessment process, there was lot of focus on the scoring to get it 'right' and some participants were finding it challenging to look beyond their organisation and assess the Alliance. Hence the process took longer than expected and the ALT was able to complete self-assessment on the first six elements rather than the total of 10 . The ALT was interested in seeing the final version of the maturity matrix rather than a version following testing with them.

\subsubsection{Alliance leadership team three feedback}

The ALT agreed that the key elements provided adequate coverage of the areas considered important to the Alliance and that the maturity matrix acted as a resource to create stimulus for change. However, the Alliance did not feel inspired to change to shift their maturity along the scale.

Certainly, a useful exercise to go through and it makes you think and reflect on where you've got to and where you might go to next.

I don't know if it made me change or do anything different. It made me aware of where there needs to be a lot of emphasis, but I don't think it's going to change anything.

Yes, in the most part, we went through the tool and felt like we were doing reasonably okay. There was no sort of burning things that we felt wow, we're really doing terribly in this place.

\section{Maturity scale content}

Suggestions to improve was directed towards the maturity matrix as a tool:

- It needs to be clear in the introduction that the maturity matrix is for the Alliance to use. 
I think a clear focus on the Alliance, otherwise it gets too confusing when you're trying to answer it and that will then just confuse things. And actually, asking it, making sure - Alliance, Alliance, Alliance thinking.

I think its value is really a drive for alliancing conversation and I think it would be really valuable to have a tool like this that really creates framework for Alliances to develop against and to use. But I think there are many tools for many reasons for many things and I think alliancing deserves a focus and an effort because we use lots of different frameworks across the rest of our systems where they're inside our PHOs or beside our provider organisations or across our whole system. I think its place is really valuable to us.

- More rigorous testing of the maturity matrix was needed, including national benchmarking, to ensure national consistency in the assessment process across all the Alliances. This will enable the Alliances to share their results with others and see how they compare with their peers.

I think there'd be an interest as well if the project is successful overall and the tool does get wider use, to see if there's some sort of benchmarking exercise that could be done too. So not only reflect on our own progress but see how we compare with our peers around the country.

It needs to become more sophisticated. The questions need to drop out or be modified, some will need to come in. But the overriding principle of what it can be used for is a really good one.

- To have one criterion per maturity scale, that is, one point per box. The ALT found it difficult to score where there was more than one point in the maturity scale box.

I think most of the explanations there were reasonably easy to follow. We then went onto number two and they were a bit harder, we struggled a bit more. So, most of those were around commitment to the Treaty and we did struggle a bit more I think, to rate ourselves on those.

It was reasonably easy, however there was some difficulty with some of the definitions and understanding what it was referring to. And maybe also useful to have some clarification around where there's three points in a box, because does that mean your sort of achievement looks like something along these lines or does 
it mean you need to have achieved all three of these or you need to have achieved two of these three. Sort of clarify that sort of question.

- $\quad$ To remove the scores, which will take the focus away from getting the 'right' score and more focus on improvement.

As a suggestion for the tool, remove the numbers. Because you're not a two or three, the power is in the words and the discourse and what you'll end up with is people ending up with is people averaging across three of the sections and say, we're a 2.33 and that is absolutely meaningless. That's what happens if you put numbers on these sorts of scales that people do that. So, if you take the numbers off then people have to use words and the words mean something.

\section{My observations}

The experience with this ALT was similar to that of the first one. On arrival I noticed the room was set up in a board room layout, with light snacks and drinks available for meeting attendees. As the attendees started arriving, by the social nature of the conversations, it was apparent that members of this ALT were familiar with each other and had a positive relationship. The energy level in the room was positive and vibrant with lots of discussion occurring. The enthusiasm at this testing was in between the first and the second ALTs. This is a mature and functional Alliance but there were indications of the ALT been DHB and hospital dominated because these members contributed more than others. There was a feeling of over-confidence and an eagerness to be an exemplar Alliance for example, there were elements that the ALT scored low but when asked if the maturity matrix stimulated them to think of improvements, they said no. The chair of the ALT and most participants were keen and enthusiastic in the testing process. However, there were signs of power imbalance between members as a few DHB members lacked interest in the process or using the process for improvement. Some of the DHB members were interested in benchmarking against peers and a moderation process to ensure national consistency and using the maturity matrix for judgement of performance.

\subsection{Feedback from Māori Health Strategy and Policy team at the MoH}

The Māori Strategy and Policy team at the $\mathrm{MoH}$ reviewed version five of the maturity matrix (following testing with ALTs) from an equity and Te Tiriti perspective and provided feedback. Overall, their feedback was positive and commended on equity and Te Tiriti embedded in the maturity matrix, both as key elements and as indicators of maturity. They commented on the 
necessity of a maturity matrix such as this to increase the understanding and responsiveness of health system leaders and Alliances towards fulfilling their obligation to Te Tiriti and improving Māori health outcomes.

The team provided the following specific feedback to strengthen the descriptions in the maturity matrix and to strengthen the alignment with Whakamaua.

Key element 2 - Commitment to Te Tiriti o Waitangi

- Clarify that Iwi and Māori both (Tangata Whenua) are Te Tiriti partners as not all Māori belong to an Iwi. This clarification applies to health providers as well, which are both Māori community and Iwi-led organisations.

- Voting right for Māori should be reflected in the 'established' stage rather than the 'excellence' stage as it is what good looks like and should be expected as a norm (rather than an exemplar).

- The outcome descriptor should aim to address and eliminate institutional racism and not just aim to reduce. The latter implies a level of tolerance to racism in the health system.

- In the first indicator, the maturity scale should reflect that historical contexts for institutional racism and injustices needs to be understood at the 'beginning' stage, at the 'emerging' stage the Alliance needs to acknowledge and be aware of their role to address institutional racism, and then addressed and eliminated at the 'established' stage. The 'excellence' stage could then reflect creating equitable health system by design that includes principles of options and active protection.

- The second indicator should reflect the context of Te Tiriti in modern day health system, that is, understanding and addressing biculturalism (Māori and Pākehā) in a multiculturalism context. The 'excellence' stage for equity indicator includes the system's ability to conduct intersectional analysis to consider contextual differences such as, socioeconomic status, disability, age, gender, and number and severity of health conditions, as highlighted in the summary of literature on equity [14].

- $\quad$ For the third indicator, Alliances need to be aware of their policy obligation to respond to five Te Tiriti principles with actions that will lead to improved health outcomes. Alliances also need to continually monitor, review and document how well they are responding to Te Tiriti based actions. 


\section{Key element 4-Involved people, whānau and community}

- The maturity scale for Te Tiriti indicator should show that co-design must be about centring the user as the designer so Māori solutions to complex problems that disproportionately affect Māori communities are valued. This would mean that Tangata Whenua would lead the design of solution, they would decide who needs to be around the table, who the insights gathering will take place with, they would decide how to interpret the results, frame the results, and would have the power to choose the best solution for their community. So, the 'beginning' stage would start with informing Tangata Whenua of the problem and the solution (tokenistic consultation), the 'emerging' stage would reflect consultation on draft proposals and the 'established' stage would mean collaboration with the design and development of the solutions. The 'excellence' stage would reflect Tangata Whenua as co-designers from start to finish and with decision-making rights over the final solution. This approach reflects Te Arawhiti's engagement framework [195].

\section{Key element 5 - Integrated health information}

- Te Tiriti indicator maturity scale could be clarified to reflect Alliance having access to: basic data at national level (by age and gender) at the 'beginning' stage; broad ad hoc data disaggregated by ethnicity with recognition of Māori data sovereignty at the 'emerging' stage; a shift away from ad hoc data collection and analysis by ethnicity to routine, mandatory reporting and monitoring by ethnicity at the 'established' stage; and use of Integrated Data Infrastructure (IDI) to connect health and social data at the 'excellence' stage.

Key element 6-Analytic capability

- Te Tiriti indicator maturity scale for the 'excellence' stage could be strengthened to reflect the Alliances' ability to become more intuitive to the future needs of Māori for the next 1015 years.

\section{Key element 8 - Intelligent commissioning}

- The equity and Te Tiriti indicators could reflect vertical equity or proportionate universalism, as highlighted in the summary of literature on equity [14].

- $\quad$ For Te Tiriti indicator, maturity scale could be clarified to reflect prioritisation of resources in proportion to Māori population need and risk so there is a tailored and targeted 
approach to actively protecting Māori health and wellbeing, including protecting Māori and Iwi-led health providers, as highlighted in the summary of literature on equity [14].

Key element 9- Understanding of equity

- The Te Tiriti indicator could match the equity indicator from the commitment to Te Tiriti element above (key element 2) and reflect vertical equity or proportionate universalism [14].

Key element 10 - Continuous improvement

- The maturity scale for Te Tiriti indicator could include establishment of Māori measures of health and wellbeing as defined by Iwi and Māori. It is important that measures are centred on Māori and they are empowered to define their own priorities for wellbeing.

Feedback from the team was incorporated in the maturity matrix and shared again to confirm an accurate reflection of the feedback. This was confirmed by the team.

\subsection{Chapter conclusion}

This chapter answered the second research question and presented the maturity matrix that was constructed with health system leaders and tested with three ALTs.

The maturity matrix was designed to improve the understanding of the programme architecture to successfully implement LST initiatives for the New Zealand Alliances. The self-assessment process supports conscious and deliberate learning so Alliances could understand the interconnected nature of the key elements that support successful implementation of LST initiatives.

The maturity matrix was constructed using complex adaptive system ideas to be simple and practical for Alliances to use and specific enough to enable self-assessment, but generic enough for local adaptability taking into consideration local context.

The maturity scale enables Alliances to assess where they are on the scale for each element; and identify areas for improvement. A regular self-assessment process will create feedback loops and support the notion of a learning system for the New Zealand health system.

Testing of the maturity matrix with three ALTs suggested that it was functioning as a learning tool; helped Alliances see where they were on the improvement journey; helped Alliances 
identify areas of improvement; acted as a resource to create a stimulus for change; and was easy and practical to use.

The maturity matrix incorporated feedback from the MoH Māori Strategy and Policy team to strengthen Te Tiriti and equity perspectives. Table 10.3 presents the final version of the maturity matrix.

This research broke new ground with the creation of a national tool in the form of a selfassessment maturity matrix using the knowledge of senior system leaders to support the New Zealand health system. The maturity matrix described different stages of maturity for the key elements so Alliances could assess their maturity against a nationally defined scale and identify areas of development should they aspire to improve their maturity. The maturity matrix filled an important gap in the New Zealand health system for successful implementation of LST initiatives to achieve long-term system transformation outcomes.

My findings showed that the maturity matrix is a helpful resource to create a stimulus for change and may act as a compass for Alliances to see where they are on the improvement journey. Not all Alliances may choose to improve themselves along the maturity scale, however, the discussion facilitates conscious and deliberate learning and creates feedback loops. The discussion may also reveal contextual factors in which the Alliance may be operating.

These feedback loops are important that may lead to changes in the behaviour of Alliance members to embrace 'new power' values such as working collectively rather than individually to improve health outcomes for their population. 


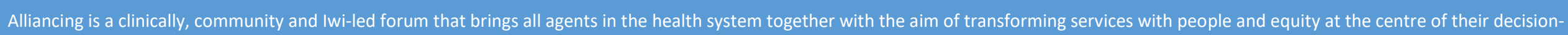

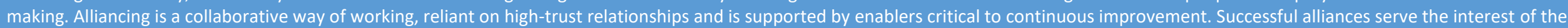
community while preserving the autonomy of multiple organisations and services present in a complex system.

\begin{tabular}{|c|c|c|c|c|c|}
\hline \multirow{2}{*}{$\begin{array}{l}\text { Outcome descriptor (what } \\
\text { does the established } \\
\text { element demonstrate?) }\end{array}$} & \multirow{2}{*}{$\begin{array}{l}\text { Indicators (What will show } \\
\text { this?) }\end{array}$} & \multicolumn{4}{|c|}{ Maturity scale - what we expect to see at each stage } \\
\hline & & Beginning & Emerging & $\begin{array}{l}\text { Established (this is what good looks } \\
\text { like) }\end{array}$ & $\begin{array}{l}\text { Excellence (outstanding eg health and } \\
\text { social integration) }\end{array}$ \\
\hline \multirow{3}{*}{$\begin{array}{l}\text { System leaders } \\
\text { demonstrate distributed } \\
\text { leadership through a } \\
\text { broad and inclusive } \\
\text { alliance membership. } \\
\text { There is high trust, shared } \\
\text { vision and sustained } \\
\text { commitment from all } \\
\text { alliance members }\end{array}$} & $\begin{array}{l}\text { Alliance is structurally embedded } \\
\text { in the system (accountable and } \\
\text { visible) }\end{array}$ & $\begin{array}{l}\text { Recognition and } \\
\text { acknowledgement of the } \\
\text { need to have a shared vision }\end{array}$ & $\begin{array}{l}\text { Shared vision and goals, } \\
\text { roles, responsibilities, and } \\
\text { accountability understood }\end{array}$ & $\begin{array}{l}\text { Alliance is structurally embedded in } \\
\text { the system with clear mandate, } \\
\text { accountability and dedicated } \\
\text { resources }\end{array}$ & $\begin{array}{l}\text { High trust and shared vision are } \\
\text { sustained with changes in membership } \\
\text { and evolution }\end{array}$ \\
\hline & $\begin{array}{l}\text { The manner in which all priority } \\
\text { populations have input into } \\
\text { decision making of the alliance }\end{array}$ & $\begin{array}{l}\text { Little or no evidence of input } \\
\text { Tokenism or lip-service }\end{array}$ & $\begin{array}{l}\text { Some populations influence } \\
\text { decision-making }\end{array}$ & $\begin{array}{l}\text { Multiple populations influence } \\
\text { decision-making via different } \\
\text { mechanisms } \\
\text { Evidence of innovation }\end{array}$ & $\begin{array}{l}\text { Alliance reflects local community and } \\
\text { includes social sector partners }\end{array}$ \\
\hline & $\begin{array}{l}\text { Depth of commitment to } \\
\text { partnership with Māori in all } \\
\text { aspects of alliance activity }\end{array}$ & $\begin{array}{l}\text { Māori representation (not } \\
\text { partnership) in alliance }\end{array}$ & $\begin{array}{l}\text { Key Māori/Iwi leadership and } \\
\text { partnership at the alliance within } \\
\text { the context of Te Tiriti o } \\
\text { Waitangi }\end{array}$ & $\begin{array}{l}\text { Te Tiriti o Waitangi principles } \\
\text { embedded in the philosophy and } \\
\text { operation of the alliance }\end{array}$ & $\begin{array}{l}\text { Full commitment to multi-sectorial and - } \\
\text { dimensional (holistic) view that enables } \\
\text { Māori aspirations e.g. whanau ora } \\
\text { approach }\end{array}$ \\
\hline \multirow{3}{*}{$\begin{array}{l}\text { Alliance members are } \\
\text { working together with a } \\
\text { collaborative approach } \\
\text { beyond their } \\
\text { organisational and } \\
\text { professional boundaries } \\
\text { towards the shared vision }\end{array}$} & $\begin{array}{l}\text { All members 'live' the alliance } \\
\text { charter }\end{array}$ & Setting up an alliance charter & $\begin{array}{l}\text { Some elements of the alliance } \\
\text { charter are demonstrated or by } \\
\text { some members }\end{array}$ & $\begin{array}{l}\text { All members of alliance 'live' the } \\
\text { alliance charter constantly and } \\
\text { consistently }\end{array}$ & $\begin{array}{l}\text { Alliance is achieving the shared vision } \\
\text { while preserving the autonomy of } \\
\text { individual providers }\end{array}$ \\
\hline & $\begin{array}{l}\text { The degree to which } \\
\text { organisations with a concern for } \\
\text { priority populations are included } \\
\text { in integrated programme } \\
\text { (design, implement and } \\
\text { evaluate) }\end{array}$ & $\begin{array}{l}\text { Little if any involvement of } \\
\text { appropriate organisations } \\
\text { (only DHBs and PHOs } \\
\text { involved) }\end{array}$ & $\begin{array}{l}\text { Beginnings of integration within } \\
\text { health alone including relevant } \\
\text { organisations that serve priority } \\
\text { population groups }\end{array}$ & $\begin{array}{l}\text { Widespread integration with } \\
\text { relevant health and disability NGOs } \\
\text { with priority population interest } \\
\text { Beginnings of de-siloed funding }\end{array}$ & $\begin{array}{l}\text { Collaboration within and beyond health } \\
\text { to appropriate priority population } \\
\text { groups including design, } \\
\text { implementation and evaluation of } \\
\text { programmes } \\
\text { Mature de-siloed funding }\end{array}$ \\
\hline & $\begin{array}{l}\text { Acknowledging Iwi/Māori as the } \\
\text { Te Tiriti o Waitangi partner }\end{array}$ & $\begin{array}{l}\text { Little or no engagement with } \\
\text { Māori community and Māori } \\
\text { and Iwi-led health providers }\end{array}$ & $\begin{array}{l}\text { Māori community and Māori and } \\
\text { Iwi-led health providers are } \\
\text { consulted during the decision- } \\
\text { making process of the alliance }\end{array}$ & $\begin{array}{l}\text { Established Māori partners (Iwi, } \\
\text { hapū, providers etc) actively } \\
\text { involved in the decision-making } \\
\text { process of the alliance, including a } \\
\text { voting right }\end{array}$ & $\begin{array}{l}\text { Māori/Iwi are leading the decision- } \\
\text { making process in the alliance } \\
\text { leadership teams, including Māori and } \\
\text { Iwi-led health and social care providers }\end{array}$ \\
\hline
\end{tabular}

Areas to work on: 
Recognising the principles of Te Tiriti o Waitangi through equity and active protection to achieve equitable health outcomes for Māori; culturally appropriate health care that recognises and supports Māori models of care, and working in partnership with Māori in the governance, design, delivery and monitoring of health services [101].

\begin{tabular}{|c|c|c|c|c|c|}
\hline \multirow{2}{*}{$\begin{array}{l}\text { Outcome descriptor } \\
\text { (what does the } \\
\text { established element } \\
\text { demonstrate?) }\end{array}$} & \multirow{2}{*}{$\begin{array}{l}\text { Indicators (What will show } \\
\text { this?) }\end{array}$} & \multicolumn{4}{|c|}{ Maturity scale - what we expect to see at each stage } \\
\hline & & Beginning & Emerging & $\begin{array}{c}\text { Established (this is what good } \\
\text { looks like) }\end{array}$ & $\begin{array}{l}\text { Excellence (outstanding e.g. } \\
\text { health and social integration) }\end{array}$ \\
\hline \multirow{3}{*}{$\begin{array}{l}\text { Alliance is working in } \\
\text { partnership with } \\
\text { Tangata Whenua in } \\
\text { the governance, } \\
\text { design, delivery and } \\
\text { monitoring of Māori } \\
\text { health outcomes. } \\
\text { Institutional racism is } \\
\text { acknowledged and } \\
\text { actions to eliminate } \\
\text { are prioritised }\end{array}$} & $\begin{array}{l}\text { Decision-making processes } \\
\text { recognise, acknowledge and } \\
\text { correct for the impact and } \\
\text { role of institutional racism }\end{array}$ & $\begin{array}{l}\text { Understanding of } \\
\text { historical contexts and } \\
\text { injustices }\end{array}$ & $\begin{array}{l}\text { Explicit acknowledgement } \\
\text { of Te Tiriti responsibilities } \\
\text { and aware of Alliance's role } \\
\text { to take proactive steps to } \\
\text { address institutional racism }\end{array}$ & $\begin{array}{l}\text { Alliance is taking actions to } \\
\text { address institutional bias and } \\
\text { improving Māori health } \\
\text { outcomes in partnership with } \\
\text { Tangata Whenua }\end{array}$ & $\begin{array}{l}\text { Alliance work programme } \\
\text { reflects an equitable system by } \\
\text { design that includes principles } \\
\text { of options and active protection }\end{array}$ \\
\hline & $\begin{array}{l}\text { Degree to which Te Tiriti is } \\
\text { understood in the modern- } \\
\text { day health system (i.e. } \\
\text { biculturalism in a multi- } \\
\text { culturalism context) }\end{array}$ & $\begin{array}{l}\text { Understanding of root } \\
\text { causes of inequities } \\
\text { within the context of Te } \\
\text { Tiriti }\end{array}$ & $\begin{array}{l}\text { Alliance is able to conduct } \\
\text { basic analysis to understand } \\
\text { inequities for Māori (i.e. by } \\
\text { age and gender) }\end{array}$ & $\begin{array}{l}\text { Alliance is using inter-sectional } \\
\text { analysis to drive key actions to } \\
\text { address inequities within the } \\
\text { context of Te Tiriti }\end{array}$ & $\begin{array}{l}\text { Evidence of re-prioritisation of } \\
\text { activities and resources for } \\
\text { Māori within the context of Te } \\
\text { Tiriti, which leads to } \\
\text { documented improved health } \\
\text { outcomes for Māori and active } \\
\text { protection of Māori and Iwi-led } \\
\text { health providers }\end{array}$ \\
\hline & $\begin{array}{l}\text { Acknowledging Iwi/ Māori } \\
\text { (Tangata Whenua) as Te } \\
\text { Tiriti partner }\end{array}$ & $\begin{array}{l}\text { Recognition of Te Tiriti } \\
\text { responsibilities for the } \\
\text { health system }\end{array}$ & $\begin{array}{l}\text { Acknowledgement and } \\
\text { awareness of Alliance's } \\
\text { obligation to respond to Te } \\
\text { Tiriti principles }\end{array}$ & $\begin{array}{l}\text { Key actions are aligned with } \\
\text { five principles of Te Tiriti: self- } \\
\text { determination, equity, active } \\
\text { protection, partnership and } \\
\text { options for Māori models of } \\
\text { care }\end{array}$ & $\begin{array}{l}\text { Alliance is continually } \\
\text { monitoring, reviewing, and } \\
\text { reporting on how well they are } \\
\text { responding to actions based on } \\
\text { five Te Tiriti principles }\end{array}$ \\
\hline
\end{tabular}

Areas to work on:

\section{Indicators (What will show this?)} gnise, acknowledge and correct for the impact and Te Tiriti responsibilities Alliance is able to conduct basic analysis to understand nequities for Māori (i.e. by biculturalism in a multicauses of inequiti within the context of $\mathrm{Te}$ care stitutional bias and Tangata Whenua Tiriti, which leads to ocumented improved health es for Māori and active health providers

Aliance is continually reporting on how well they are responding to actions based on 
Key element 3 - Clinical leadership and involvement

A system through which health care professionals provide leadership and system oversight with a focus on continuous quality improvement to create an environment for evidencebased clinical practice and team-based approaches to care delivery [191].

\begin{tabular}{|c|c|c|c|c|c|}
\hline \multirow{2}{*}{$\begin{array}{l}\text { Outcome descriptor } \\
\text { (what does the } \\
\text { established element } \\
\text { demonstrate?) }\end{array}$} & \multirow{2}{*}{$\begin{array}{l}\text { Indicators (What will show } \\
\text { this?) }\end{array}$} & \multicolumn{4}{|c|}{ Maturity scale - what we expect to see at each stage } \\
\hline & & Beginning & Emerging & $\begin{array}{c}\text { Established (this is what good } \\
\text { looks like) }\end{array}$ & $\begin{array}{l}\text { Excellence (outstanding e.g. } \\
\text { health and social integration) }\end{array}$ \\
\hline \multirow{3}{*}{$\begin{array}{l}\text { LST initiatives are } \\
\text { designed and } \\
\text { implemented with or } \\
\text { are led by the clinical } \\
\text { leaders and health } \\
\text { care professionals. } \\
\text { Recommended } \\
\text { solutions are accepted } \\
\text { and implemented by } \\
\text { the operational } \\
\text { leaders }\end{array}$} & $\begin{array}{l}\text { Operational and clinical } \\
\text { leadership work as one }\end{array}$ & $\begin{array}{l}\text { Rebalancing of } \\
\text { managerial and clinical } \\
\text { input into system change } \\
\text { development }\end{array}$ & $\begin{array}{l}\text { Trust model in place } \\
\text { between managerial and } \\
\text { clinical input and } \\
\text { understanding of the } \\
\text { different roles }\end{array}$ & $\begin{array}{l}\text { Clinicians involved in } \\
\text { investment decisions } \\
\text { High attendance of clinical } \\
\text { leaders to Alliance meetings } \\
\text { Explicit sign off by clinicians }\end{array}$ & $\begin{array}{l}\text { Distributed leadership and } \\
\text { shared vision through honest } \\
\text { conversations, respect, } \\
\text { acknowledgement of roles, and } \\
\text { sustainable ongoing leadership }\end{array}$ \\
\hline & $\begin{array}{l}\text { The degree to which } \\
\text { clinicians and managers } \\
\text { knowledge of population } \\
\text { health data is understood } \\
\text { and actioned }\end{array}$ & $\begin{array}{l}\text { Little or no evidence of } \\
\text { population health data } \\
\text { being considered by } \\
\text { managers and clinicians }\end{array}$ & $\begin{array}{l}\text { Some evidence of } \\
\text { meaningful discussion } \\
\text { between clinicians and } \\
\text { managers about equity } \\
\text { issues }\end{array}$ & $\begin{array}{l}\text { Work programme has } \\
\text { alignment between population } \\
\text { health data and funding } \\
\text { allocation }\end{array}$ & $\begin{array}{l}\text { Clear evidence of regular } \\
\text { consideration of equity issues } \\
\text { between clinicians and } \\
\text { managers and clearly } \\
\text { documented improved health } \\
\text { outcomes }\end{array}$ \\
\hline & $\begin{array}{l}\text { Membership and way of } \\
\text { working reflects Te Tiriti } \\
\text { principles }\end{array}$ & $\begin{array}{l}\text { Little or no Māori clinical } \\
\text { leadership }\end{array}$ & $\begin{array}{l}\text { Māori clinicians and } \\
\text { managers part of clinical } \\
\text { and Alliance forums }\end{array}$ & $\begin{array}{l}\text { Evidence of Māori clinical } \\
\text { attendance, participation and } \\
\text { influence in investment } \\
\text { decisions }\end{array}$ & $\begin{array}{l}\text { Dedicated workstream for } \\
\text { Māori outcomes with multi- } \\
\text { disciplinary and -sectorial } \\
\text { approach }\end{array}$ \\
\hline
\end{tabular}

Areas to work on:

\section{Indicators (What will show Indicator
this?)}

Operational and clinical

managerial and

\section{between managerial and}

clinical input and

understanding of the

different roles

Some evidence of

meaningful discussion

managers about equity

managers part of clinical

and Alliance forums influence in investment

disciplinary and -sectoria 
Key element 4 - Involved people, whanau and community

An approach that actively involves individuals, carers, hapū, whānau, Iwi and communities in the design and delivery of health care to generate significant benefits to the health care and wellbeing of all people.

\begin{tabular}{|c|c|c|c|c|c|}
\hline \multirow{2}{*}{$\begin{array}{l}\text { Outcome descriptor } \\
\text { (what does the } \\
\text { established element } \\
\text { demonstrate?) }\end{array}$} & \multirow{2}{*}{$\begin{array}{l}\text { Indicators (What will show } \\
\text { this?) }\end{array}$} & \multicolumn{4}{|c|}{ Maturity scale - what we expect to see at each stage } \\
\hline & & Beginning & Emerging & $\begin{array}{l}\text { Established (this is what good } \\
\text { looks like) }\end{array}$ & $\begin{array}{l}\text { Excellence (outstanding eg } \\
\text { health and social integration) }\end{array}$ \\
\hline \multirow[t]{3}{*}{$\begin{array}{l}\text { People, whānau and } \\
\text { communities are } \\
\text { involved at the } \\
\text { governance and } \\
\text { decision-making } \\
\text { levels to co-design the } \\
\text { system and the } \\
\text { services }\end{array}$} & $\begin{array}{l}\text { Information is available to } \\
\text { communities to understand } \\
\text { what is important to them }\end{array}$ & $\begin{array}{l}\text { Acknowledge the need } \\
\text { to have the right people } \\
\text { around the table }\end{array}$ & $\begin{array}{l}\text { Acknowledgement that } \\
\text { engagement is broader than } \\
\text { a seat at the table, ie, it is } \\
\text { how the alliance engages } \\
\text { with its broader community } \\
\text { to prioritise and exchange } \\
\text { information }\end{array}$ & $\begin{array}{l}\text { A multi-channel engagement } \\
\text { plan in place with meaningful } \\
\text { input from individuals and } \\
\text { communities that leads them to } \\
\text { believe that the right decisions } \\
\text { are being made for services } \\
\text { they need }\end{array}$ & $\begin{array}{l}\text { Individuals and communities } \\
\text { are involved in decision-making } \\
\text { for re-prioritising services to } \\
\text { changing need }\end{array}$ \\
\hline & $\begin{array}{l}\text { Degree to which individual, } \\
\text { whanau and community } \\
\text { voice from all populations is } \\
\text { heard and acted upon in } \\
\text { decision-making }\end{array}$ & $\begin{array}{l}\text { Priority populations are } \\
\text { informed of decisions } \\
\text { through information } \\
\text { provided by the alliance }\end{array}$ & $\begin{array}{l}\text { Alliance consults priority } \\
\text { population communities on } \\
\text { draft proposals but makes } \\
\text { the final decisions }\end{array}$ & $\begin{array}{l}\text { Community representatives } \\
\text { from priority populations part } \\
\text { of alliance and are influencing } \\
\text { decision-making of the alliance }\end{array}$ & $\begin{array}{l}\text { Individuals and communities } \\
\text { from priority populations are } \\
\text { involved in co-design and } \\
\text { decision-making processes of } \\
\text { the alliance }\end{array}$ \\
\hline & $\begin{array}{l}\text { Tangata Whenua (Māori, Iwi, } \\
\text { whānau, hapū) are partners } \\
\text { in all stages of design, } \\
\text { delivery and implementation }\end{array}$ & $\begin{array}{l}\text { Tangata Whenua are } \\
\text { informed of decisions } \\
\text { through information } \\
\text { provided by the alliance }\end{array}$ & $\begin{array}{l}\text { Alliance consults Tangata } \\
\text { Whenua on draft proposals } \\
\text { but makes the final } \\
\text { decisions }\end{array}$ & $\begin{array}{l}\text { Alliances collaborates with } \\
\text { Tangata Whenua to develop } \\
\text { solutions together and Tangata } \\
\text { Whenua are involved in } \\
\text { decision-making process or } \\
\text { make joint decisions with the } \\
\text { alliance }\end{array}$ & $\begin{array}{l}\text { Alliance partners with Tangata } \\
\text { Whenua to co-design services } \\
\text { and implements decisions made } \\
\text { by Tangata Whenua for the final } \\
\text { solution }\end{array}$ \\
\hline
\end{tabular}

Areas to work on: 
Key element 5 - Integrated health information

The availability of technology, and health and social information, both at identifiable and population aggregate level, across the different parts of the system at local and national level. This readily accessible health information is responsive to needs and guides commissioning decisions.

\begin{tabular}{|c|c|c|c|c|c|}
\hline \multirow{2}{*}{$\begin{array}{l}\text { Outcome descriptor } \\
\text { (what does the } \\
\text { established element } \\
\text { demonstrate?) }\end{array}$} & \multirow{2}{*}{$\begin{array}{l}\text { Indicators (What will show } \\
\text { this?) }\end{array}$} & \multicolumn{4}{|c|}{ Maturity scale - what we expect to see at each stage } \\
\hline & & Beginning & Emerging & $\begin{array}{c}\text { Established (this is what good } \\
\text { looks like) }\end{array}$ & $\begin{array}{l}\text { Excellence (outstanding e.g. } \\
\text { health and social integration) }\end{array}$ \\
\hline \multirow{3}{*}{$\begin{array}{l}\text { DHBs can link data } \\
\text { from multiple } \\
\text { platforms that } \\
\text { includes } \\
\text { administrative and } \\
\text { social sector data at } \\
\text { the population and } \\
\text { patient identifiable } \\
\text { level and share with } \\
\text { the alliance for } \\
\text { continuous } \\
\text { improvement }\end{array}$} & $\begin{array}{l}\text { NHI linked data available to } \\
\text { meet decision-making needs } \\
\text { for the problem at hand }\end{array}$ & $\begin{array}{l}\text { Alliance is receiving basic } \\
\text { data from the } \mathrm{DHB}\end{array}$ & $\begin{array}{l}\text { DHB is supporting alliance } \\
\text { with data sharing } \\
\text { agreements with all system } \\
\text { partners }\end{array}$ & $\begin{array}{l}\text { Alliance has access to real-time } \\
\text { data from across the system, } \\
\text { both at NHI and population } \\
\text { level }\end{array}$ & $\begin{array}{l}\text { Alliance has access to the right } \\
\text { information at the right time for } \\
\text { forecasting and feedback to } \\
\text { drive continuous improvement }\end{array}$ \\
\hline & $\begin{array}{l}\text { Degree to which integrated } \\
\text { information and intelligence } \\
\text { for high priority populations } \\
\text { are explicitly considered and } \\
\text { managed routinely }\end{array}$ & $\begin{array}{l}\text { Alliance receives basic } \\
\text { data at DHB level } \\
\text { disaggregated by age, } \\
\text { gender and ethnicity }\end{array}$ & $\begin{array}{l}\text { Alliance receives ad hoc } \\
\text { integrated data at national } \\
\text { and local level, } \\
\text { disaggregated by age, } \\
\text { gender and ethnicity }\end{array}$ & $\begin{array}{l}\text { Alliance routinely receives } \\
\text { timely, useful, integrated data } \\
\text { as part of mandatory reporting } \\
\text { and monitoring by ethnicity }\end{array}$ & $\begin{array}{l}\text { Alliance has access to health } \\
\text { information that is integrated } \\
\text { with administrative and social } \\
\text { sector data to drive equity } \\
\text { actions }\end{array}$ \\
\hline & $\begin{array}{l}\text { Māori have sovereignty over } \\
\text { integrated health } \\
\text { information }\end{array}$ & $\begin{array}{l}\text { Alliance receives basic } \\
\text { data at DHB level } \\
\text { disaggregated by age, } \\
\text { gender and ethnicity }\end{array}$ & $\begin{array}{l}\text { Alliance receives ad hoc } \\
\text { integrated data at national } \\
\text { and local level, } \\
\text { disaggregated by age, } \\
\text { gender and ethnicity with } \\
\text { recognition of Māori data } \\
\text { sovereignty }\end{array}$ & $\begin{array}{l}\text { Alliance routinely receives } \\
\text { timely, useful, integrated data } \\
\text { as part of mandatory reporting } \\
\text { and monitoring by ethnicity }\end{array}$ & $\begin{array}{l}\text { Alliance has access to health } \\
\text { information that is integrated } \\
\text { with administrative and social } \\
\text { sector data to meet their Te } \\
\text { Tiriti obligations }\end{array}$ \\
\hline
\end{tabular}

Areas to work on:

sovereignty 
Key element 6 - Analytic capability

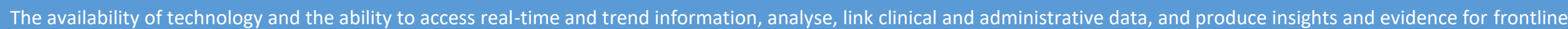
staff to measure, understand and feedback data on clinical variation and outcomes.

\begin{tabular}{|c|c|c|c|c|c|}
\hline \multirow{2}{*}{$\begin{array}{l}\text { Outcome descriptor } \\
\text { (what does the } \\
\text { established element } \\
\text { demonstrate?) }\end{array}$} & \multirow{2}{*}{$\begin{array}{l}\text { Indicators (What will show } \\
\text { this?) }\end{array}$} & \multicolumn{4}{|c|}{ Maturity scale - what we expect to see at each stage } \\
\hline & & Beginning & Emerging & $\begin{array}{l}\text { Established (this is what good } \\
\text { looks like) }\end{array}$ & $\begin{array}{l}\text { Excellence (outstanding e.g. } \\
\text { health and social integration) }\end{array}$ \\
\hline \multirow{3}{*}{$\begin{array}{l}\text { Improvement culture, } \\
\text { skills and knowledge } \\
\text { available to turn all } \\
\text { intelligence about } \\
\text { health of local } \\
\text { populations into } \\
\text { insights about } \\
\text { variation and change } \\
\text { in quality and } \\
\text { outcomes of health } \\
\text { care for alliance to } \\
\text { use for continuous } \\
\text { improvement }\end{array}$} & $\begin{array}{l}\text { Intelligence about health of } \\
\text { local populations into } \\
\text { insights about variation and } \\
\text { change in quality and } \\
\text { outcomes of health care }\end{array}$ & $\begin{array}{l}\text { Recognition that } \\
\text { intelligence is required } \\
\text { and initial discussion to } \\
\text { establish analytic needs } \\
\text { for alliance work } \\
\text { programme }\end{array}$ & $\begin{array}{l}\text { DHB invests in tools and } \\
\text { resources to produce } \\
\text { evidence for alliance to use } \\
\text { to drive decisions }\end{array}$ & $\begin{array}{l}\text { Growing sophistication on the } \\
\text { level of insights and evidence } \\
\text { available to enable alliance to } \\
\text { conduct consistent analysis } \\
\text { over time to plan, do, study and } \\
\text { act }\end{array}$ & $\begin{array}{l}\text { Alliance is able to use insights } \\
\text { and evidence for 'rear-view' } \\
\text { and for forward projection } \\
\text { Cross district learning and } \\
\text { sharing is occurring }\end{array}$ \\
\hline & $\begin{array}{l}\text { Degree to which intelligence } \\
\text { resources are allocated to } \\
\text { analysing data with an } \\
\text { equity focus }\end{array}$ & $\begin{array}{l}\text { Limited analytic capacity } \\
\text { and little understanding } \\
\text { of analysing variation or } \\
\text { measuring change for } \\
\text { high priority populations }\end{array}$ & $\begin{array}{l}\text { Appropriate analytic } \\
\text { capacity and capability to } \\
\text { support alliance with } \\
\text { intelligence to analyse } \\
\text { variation and measure } \\
\text { change for high priority } \\
\text { populations }\end{array}$ & $\begin{array}{l}\text { Insights and evidence are } \\
\text { consistently provided to } \\
\text { alliance to routinely plan, do, } \\
\text { study and act for high priority } \\
\text { populations }\end{array}$ & $\begin{array}{l}\text { Insights and evidence are } \\
\text { driving investment decisions of } \\
\text { the alliance to respond to the } \\
\text { future needs of high priority } \\
\text { populations }\end{array}$ \\
\hline & $\begin{array}{l}\text { Degree to which intelligence } \\
\text { resources are allocated to } \\
\text { meet Te Tiriti obligations }\end{array}$ & $\begin{array}{l}\text { Limited analytic capacity } \\
\text { to understand, measure } \\
\text { and routinely report } \\
\text { Māori Health outcomes }\end{array}$ & $\begin{array}{l}\text { Appropriate analytic } \\
\text { capacity and capability to } \\
\text { support alliance with } \\
\text { intelligence to understand, } \\
\text { measure and routinely } \\
\text { report Māori Health } \\
\text { outcomes }\end{array}$ & $\begin{array}{l}\text { Insights and evidence are } \\
\text { consistently provided to } \\
\text { alliance to understand, } \\
\text { measure and routinely report } \\
\text { Māori Health outcomes }\end{array}$ & $\begin{array}{l}\text { Alliance becomes intuitive to } \\
\text { the future needs of Māori for } \\
\text { the next } 10-15 \text { years }\end{array}$ \\
\hline
\end{tabular}

Areas to work on: 
Key element 7 - Dedicated resources and time

Availability of appropriate continuous resourcing such as appropriate health workforce, funding, knowledge, time, project management support, and administration support. There is an acknowledgement that transformational change needs capacity and long-term commitment.

\begin{tabular}{|c|c|c|c|c|c|}
\hline \multirow{2}{*}{$\begin{array}{l}\text { Outcome descriptor } \\
\text { (what does the } \\
\text { established element } \\
\text { demonstrate?) }\end{array}$} & \multirow{2}{*}{$\begin{array}{l}\text { Indicators (What will show } \\
\text { this?) }\end{array}$} & \multicolumn{4}{|c|}{ Maturity scale - what we expect to see at each stage } \\
\hline & & Beginning & Emerging & $\begin{array}{l}\text { Established (this is what good } \\
\text { looks like) }\end{array}$ & $\begin{array}{l}\text { Excellence (outstanding e.g. } \\
\text { health and social integration) }\end{array}$ \\
\hline \multirow[t]{3}{*}{$\begin{array}{l}\text { Sustainable and } \\
\text { dedicated resources } \\
\text { located in the right } \\
\text { place in the system for } \\
\text { change management }\end{array}$} & $\begin{array}{l}\text { Resources ring-fenced to } \\
\text { manage and support change } \\
\text { management and decision- } \\
\text { making }\end{array}$ & $\begin{array}{l}\text { Alliancing is seen as a } \\
\text { good idea but embryonic } \\
\text { form, function and } \\
\text { dedicated resources } \\
\text { relies on goodwill }\end{array}$ & $\begin{array}{l}\text { DHB recognises that } \\
\text { alliancing needs to be } \\
\text { resourced within the system } \\
\text { with funding and human } \\
\text { resources }\end{array}$ & $\begin{array}{l}\text { Alliance has an independent } \\
\text { programme office with } \\
\text { dedicated management and } \\
\text { clinical resource to achieve its } \\
\text { work programme }\end{array}$ & $\begin{array}{l}\text { Alliance programme office is } \\
\text { embedded in the system with } \\
\text { sustainable funding }\end{array}$ \\
\hline & $\begin{array}{l}\text { Degree to which equity is } \\
\text { explicitly incorporated into } \\
\text { all elements of alliance work } \\
\text { programme }\end{array}$ & $\begin{array}{l}\text { Equity mentioned in } \\
\text { alliance terms of } \\
\text { reference but no further } \\
\text { evidence of funding }\end{array}$ & $\begin{array}{l}\text { Mention of equity in alliance } \\
\text { related work with some } \\
\text { funded initiatives aimed at } \\
\text { improving equity }\end{array}$ & $\begin{array}{l}\text { Multiple funded equity actions } \\
\text { aimed at high priority } \\
\text { populations with routine } \\
\text { monitoring and reporting of } \\
\text { health outcomes }\end{array}$ & $\begin{array}{l}\text { Equity is at the centre of all the } \\
\text { alliance work programme with } \\
\text { sustained funding, and routine } \\
\text { monitoring and reporting of } \\
\text { health outcomes }\end{array}$ \\
\hline & $\begin{array}{l}\text { Core business spending is } \\
\text { challenged and re- } \\
\text { prioritised to meet Māori } \\
\text { aspirations for wellbeing }\end{array}$ & $\begin{array}{l}\text { Alliance recognises the } \\
\text { need to prioritise } \\
\text { resources for equity } \\
\text { actions to improve } \\
\text { Māori health outcomes }\end{array}$ & $\begin{array}{l}\text { Alliance has funded equity } \\
\text { actions to improve Māori } \\
\text { health outcomes }\end{array}$ & $\begin{array}{l}\text { Alliance's decisions on funding } \\
\text { and resource allocations are } \\
\text { equity based within the context } \\
\text { of Te Tiriti }\end{array}$ & $\begin{array}{l}\text { Alliance challenges current } \\
\text { investment decisions to support } \\
\text { hauora Māori models of care }\end{array}$ \\
\hline
\end{tabular}

Areas to work on:

Māori health outcomes 


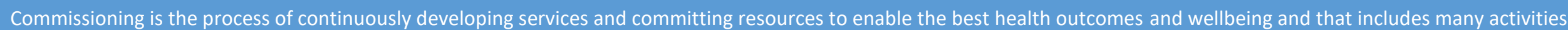
ranging from health needs assessment, cultural paradigms and development of pathways to service specification and contract management or procurement, underpinned by continuous improvement.

\section{Outcome descriptor}

(what does the

established element

demonstrate?)

\section{Continuous strategic}

and operational

planning is

underpinned by

continuous

improvement that

enables the alliance

to make evidence-

based decisions

Areas to work on:
Indicators (What will show

this?)

Clear evidence for alliance

decision-making for

commissioning of health

services

Prioritisation of resources in proportion to high priority populations' need and risk with a targeted approach to equity

Prioritisation of resources in proportion to Māori need and risk with a tailored and target approach to actively protecting Māori wellbeing
Maturity scale - what we expect to see at each stage

\begin{tabular}{|c|c|c|c|}
\hline Beginning & Emerging & $\begin{array}{l}\text { Established (this is what good } \\
\text { looks like) }\end{array}$ & $\begin{array}{l}\text { Excellence (outstanding e.g. } \\
\text { health and social integration) }\end{array}$ \\
\hline $\begin{array}{l}\text { Alliance recognise the } \\
\text { need for evidence-based } \\
\text { decision-making for } \\
\text { commissioning of health } \\
\text { services }\end{array}$ & $\begin{array}{l}\text { Alliance has access to } \\
\text { limited evidence to inform } \\
\text { their decision-making for } \\
\text { current and future } \\
\text { commissioning of health } \\
\text { services }\end{array}$ & $\begin{array}{l}\text { Alliance has access to } \\
\text { comprehensive evidence to } \\
\text { inform some of their decision- } \\
\text { making for current and future } \\
\text { commissioning of health } \\
\text { services }\end{array}$ & $\begin{array}{l}\text { Alliance is using the evidence to } \\
\text { inform all their decision-making } \\
\text { for current and future } \\
\text { commissioning of health } \\
\text { services }\end{array}$ \\
\hline $\begin{array}{l}\text { Alliance decisions are } \\
\text { not based on available } \\
\text { intelligence and there is } \\
\text { no consideration of } \\
\text { priority populations } \\
\text { (perpetuating status } \\
\text { quo) }\end{array}$ & $\begin{array}{l}\text { Some decisions are based } \\
\text { on evidence to prioritise } \\
\text { resources in proportion to } \\
\text { high priority populations' } \\
\text { need and risk }\end{array}$ & $\begin{array}{l}\text { Equity is at the centre of all } \\
\text { decision-making for current and } \\
\text { future commissioning of health } \\
\text { services }\end{array}$ & $\begin{array}{l}\text { Continuous use of evidence, } \\
\text { monitoring of actions, and } \\
\text { reporting to show that current } \\
\text { and future commissioning is } \\
\text { proportional to high priority } \\
\text { populations' need and risk }\end{array}$ \\
\hline $\begin{array}{l}\text { Alliance acknowledging } \\
\text { that resources to Māori } \\
\text { will be different }\end{array}$ & $\begin{array}{l}\text { Alliance is identifying } \\
\text { resources in proportion to } \\
\text { Māori need and risk to } \\
\text { improve Māori wellbeing }\end{array}$ & $\begin{array}{l}\text { Alliance is recommending to } \\
\text { DHB to de-invest in other areas } \\
\text { and re-invest into Māori and } \\
\text { prioritise new funding to Māori }\end{array}$ & $\begin{array}{l}\text { Alliance is recommending } \\
\text { commissioning decisions based } \\
\text { on kaupapa Māori models and } \\
\text { protecting Māori and Iwi-led } \\
\text { health providers }\end{array}$ \\
\hline
\end{tabular}


Key element 9 - Understanding of equity

Equity recognises different people with different levels of advantage require different approaches and resources to get equitable health outcomes [193]. Emphasis is given to Pacific and other high opportunity populations such as those with mental health conditions and with disability.

\begin{tabular}{|c|c|c|c|c|c|}
\hline \multirow{2}{*}{$\begin{array}{l}\text { Outcome descriptor } \\
\text { (what does the } \\
\text { established element } \\
\text { demonstrate?) }\end{array}$} & \multirow{2}{*}{$\begin{array}{l}\text { Indicators (What will show } \\
\text { this?) }\end{array}$} & \multicolumn{4}{|c|}{ Maturity scale - what we expect to see at each stage } \\
\hline & & Beginning & Emerging & $\begin{array}{l}\text { Established (this is what good } \\
\text { looks like) }\end{array}$ & $\begin{array}{l}\text { Excellence (outstanding e.g. } \\
\text { health and social integration) }\end{array}$ \\
\hline \multirow{3}{*}{$\begin{array}{l}\text { Alliance is able to } \\
\text { identify and } \\
\text { understand the } \\
\text { variation in health } \\
\text { care in their } \\
\text { population, identify } \\
\text { populations groups } \\
\text { that consistently } \\
\text { experience poor } \\
\text { health outcomes and } \\
\text { re-prioristise activities } \\
\text { and funding to } \\
\text { address health } \\
\text { inequities }\end{array}$} & $\begin{array}{l}\text { Every Alliance decision has } \\
\text { an equity focus based on } \\
\text { information that is easy to } \\
\text { understand }\end{array}$ & $\begin{array}{l}\text { Equity mentioned but } \\
\text { not yet widely } \\
\text { understood or explicitly } \\
\text { addressed }\end{array}$ & $\begin{array}{l}\text { Start to see equity as key } \\
\text { part of regular Alliance } \\
\text { conversation including } \\
\text { discussion on how to } \\
\text { address inequities }\end{array}$ & $\begin{array}{l}\text { Alliance work programme and } \\
\text { investment decisions explicitly } \\
\text { address health inequities and } \\
\text { undertake routine monitoring } \\
\text { of effectiveness of change to } \\
\text { close gaps }\end{array}$ & $\begin{array}{l}\text { Alliance works collaboratively } \\
\text { with social sector partners to } \\
\text { reduce inequities including a } \\
\text { focus on social determinants of } \\
\text { health }\end{array}$ \\
\hline & $\begin{array}{l}\text { Degree to which Alliance } \\
\text { uses proportionate } \\
\text { universalism approach }\end{array}$ & $\begin{array}{l}\text { Alliance understands } \\
\text { that actions and } \\
\text { resources need to be } \\
\text { proportionate to need } \\
\text { and level of } \\
\text { disadvantage in their } \\
\text { population }\end{array}$ & $\begin{array}{l}\text { Alliance identifies high } \\
\text { priority populations in their } \\
\text { district }\end{array}$ & $\begin{array}{l}\text { Alliance prioritises their work } \\
\text { programme and resources } \\
\text { proportionate to the need of } \\
\text { their high priority populations }\end{array}$ & $\begin{array}{l}\text { Evidence of re-prioritisation of } \\
\text { activities and resources Pacific } \\
\text { and other population groups } \\
\text { that experience health } \\
\text { inequities, which leads to } \\
\text { documented improved health } \\
\text { outcomes }\end{array}$ \\
\hline & $\begin{array}{l}\text { Degree to which Te Tiriti is } \\
\text { understood in the modern- } \\
\text { day health system (i.e. } \\
\text { biculturalism in a multi- } \\
\text { culturalism context) }\end{array}$ & $\begin{array}{l}\text { Understanding of root } \\
\text { causes of inequities } \\
\text { within the context of Te } \\
\text { Tiriti }\end{array}$ & $\begin{array}{l}\text { Alliance is able to conduct } \\
\text { basic analysis to understand } \\
\text { inequities for Māori (i.e. by } \\
\text { age and gender) }\end{array}$ & $\begin{array}{l}\text { Alliance is using inter-sectional } \\
\text { analysis to drive key actions to } \\
\text { address inequities within the } \\
\text { context of Te Tiriti }\end{array}$ & $\begin{array}{l}\text { Evidence of re-prioritisation of } \\
\text { activities and resources for } \\
\text { Māori within the context of Te } \\
\text { Tiriti, which leads to } \\
\text { documented improved health } \\
\text { outcomes for Māori and active } \\
\text { protection of Māori and Iwi-led } \\
\text { health providers }\end{array}$ \\
\hline
\end{tabular}

\section{Areas to work on:}


Key element 10 - Continuous improvement

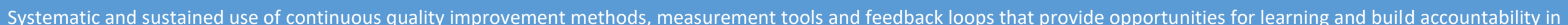
the system.

\section{Outcome descriptor}

(what does the

established element

demonstrate?)

Improvement culture, skills, knowledge and experience of using quality improvement science and

techniques to drive

measurable impact in

quality and outcomes

of the health system

by the alliance

jou

\section{Areas to work on:}

\section{Indicators (What will show this?)}

Specific resource and funding put aside for alliances to use for

continuous improvement

Degree to which alliance can demonstrate equity gap is

reducing as a result of

improvement activities (no

minimum targets)

Māori aspirations are

integrated in improvement journey

\begin{tabular}{|l|l}
\hline \\
imp \\
ing \\
ing \\
\hline
\end{tabular}

Beginning

Recognition that

conscious investment in

continuous

improvement is essential

Improvement activities

have had no effect on

inequities or worsened

\section{Awareness that}

traditional improvement

methods and models are

not always appropriate

for Māori

Maturity scale - what we expect to see at each stage

Emerging

Alliance has resources for

continuous improvement

Existing or new

improvement activities

consider inequity, but

effects are unclear or

inconsistent

Identification of Māori

improvement models e.g. Te

whare tapa whā model

\section{Established (this is what good} looks like)

Continuous improvement

activities are part of every

decision and implementation

with continued feedback loops

Existing and new improvement activities consistently focused

on improvements in equity

Māori knowledge is respected,

and Māori measures of

wellbeing are defined by

Tangata Whenua
Excellence (outstanding e.g.

health and social integration

Continuous improvement builds accountability in the system to measure progress towards achieving the Triple Aim

Accountability in the system through routine measuring, monitoring and reporting of activities to reduce inequities for high priority populations

Alliance is empowering Māori to define their own priorities for wellbeing and realise their aspirations 
Alliance Action Plan (post self-assessment)

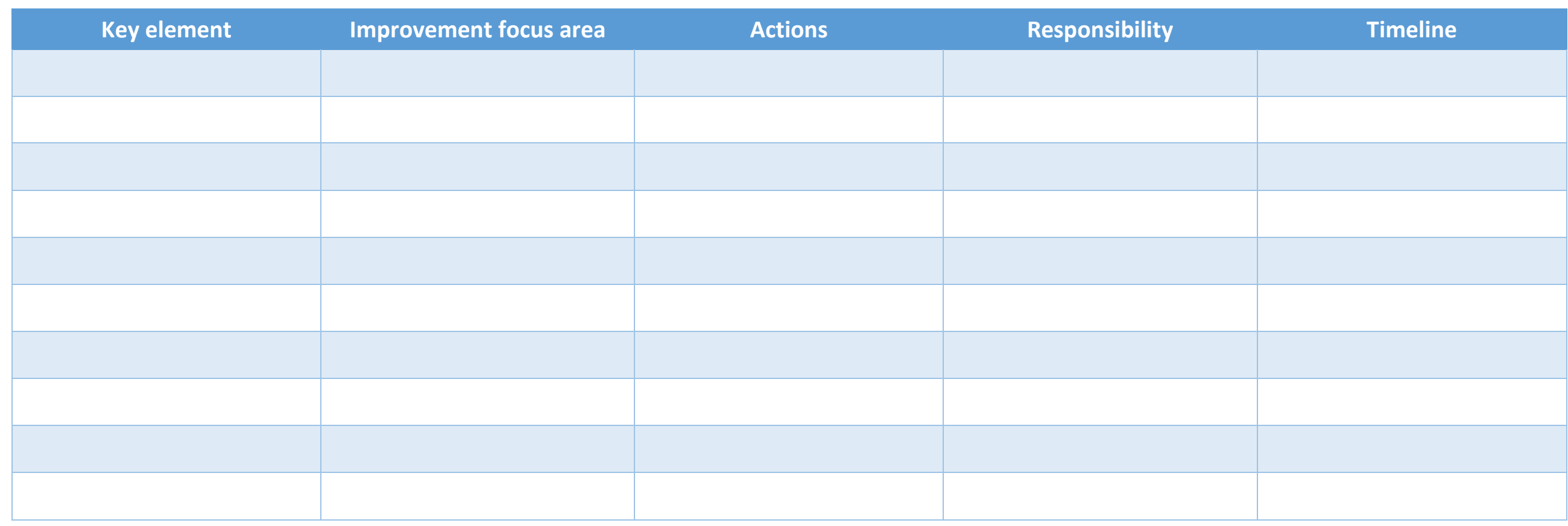

Table 10.3: Maturity Matrix for New Zealand Alliances - a self-assessment tool to support large-scale change in health system (final version) 

INITIATIVES

Having established the key elements that need to be present to increase the chances of success with implementation of LST initiatives in a New Zealand context (Chapter 9), I wanted to drill down to investigate how these elements worked in different contexts. I used the realist framing of $\mathrm{CMO}$ with $\mathrm{C}$ being the contextual factors in which Alliances operate in, $\mathrm{M}$ being the underlying reasoning of health system agents involved in the implementation of LST initiatives, and O being the variations in the intermediate outcomes for successful implementation of LST initiatives. Five methods were used to test and refine the $\mathrm{CMO}$ theories: initial propositions based on my insights from the SLM programme (phase 1); a literature review (phase 2); interviews (phase 3); workshops (phase 4); and an online survey (phase 5).

The chapter starts with my insights gleaned from the implementation of the SLM programme that formed the initial propositions of the contexts and mechanisms that caused variation in districts' attempt to successfully implement the programme.

\subsection{Initial propositions (phase 1 - theory gleaning)}

My initial propositions were gleaned from insights gained through leading the implementation of the SLM programme for three years (described in detail in section 9.1). In phase 1 of my research, I theorised that six local contextual factors influenced the implementation of the SLM programme and caused variation in districts' attempt to successfully implement the SLM programme. The local contextual factors were:

- $\quad$ distributed leadership from DHBs

- $\quad$ a history of working together at the district level

- $\quad$ quality of relationships between DHBs and PHOs

- the maturity of Alliances

- $\quad$ the capacity and capability of Alliances

- $\quad$ the continuous improvement culture at DHBs and PHOs.

Distributed leadership is where key leadership functions, which includes making important decisions, are shared with a set of people and decisions emerge from a collective process through the interactions of multiple actors $[1,196,197]$.

I theorised that the 'family of mechanisms' that were triggered by these contextual factors were: 
- a commitment from Alliance members to implement the SLM programme

- $\quad$ high trust relationships among senior operational and clinical leaders in the district

- $\quad$ an agreement to a shared vision and goals for the district

- $\quad$ active support from senior leaders at DHBs and PHOs for implementation of the SLM programme

- recognition of the importance of and investment in improvement capacity and capability.

I theorised that one national context influenced the implementation of the SLM programme:

- the use of 'new power' values of collaborative design with a continuous improvement approach and implementation through Alliances.

The mechanism that was triggered by this contextual factor was:

- the buy-in from the sector, in particular clinical leaders and frontline health care professionals with the implementation of the SLM programme, which made the programme successful. Buy-in was demonstrated by clinical leaders and staff prioritising the time to engage, their willingness for continuous learning to improve the quality of the SLM plans, their advocacy of the SLM programme to their peers, managers, the Minister of Health and the senior leaders in the $\mathrm{MoH}$, and their perseverance with working in a collaborative way even when local relationships were fraught.

\subsection{Testing of initial propositions}

The initial propositions were used with research participants to explore how key elements worked in different contexts to influence the successful implementation of LST initiatives using the realist research design outlined in

Figure 7.1 (Chapter 7) of this thesis. I used the teacher-learner method; first, as a teacher to introduce the initial propositions and then as a learner where I sought participants' perspectives. I used this approach in three research phases; phase 3 (interviews), phase 4 (workshops) and phase 5 (the online survey), and tested perspectives and emerging theories discovered from preceding phases with subsequent phases. For example, I tested emerging theories from interviews conducted prior to the workshops with the workshop participants, tested emerging theories from workshops with subsequent interviews, and then tested revised propositions from interviews and workshops with online survey participants. I then synthesised the CMO configurations from all five research phases. 
Participants in the study were senior health system leaders from New Zealand and overseas that had experience leading LST initiatives. Some of the New Zealand participants in the interviews and the workshops were involved in the development and implementation of the SLM programme. The online survey participants were DHB and PHO employees who were either leading or involved in the implementation of the SLM programme. These participants had a great deal of on-the-ground knowledge about what works to bring about change in the health system and provided information that revealed mid-range CMO theories for successful implementation of LST initiatives.

Sections 11.3 and 11.4 consolidate findings from interviews, workshops and the online survey. Section 11.3 discusses the CMO theories influenced by local contexts and section 11.4 discusses the CMO theories influenced by national contexts. Each sub-section discusses a context confirmed by research participants and the underlying, unseen mechanisms triggered by the context leading to variations in intermediate outcomes for successful implementation of LST initiatives. Each sub-section ends with a summary of mid-range CMO theories for successful implementation of LST initiatives for the context.

\subsection{Local contexts and mechanisms}

\subsubsection{History of working together and quality of relationships}

A history of working together, whether it be among senior leaders or among frontline staff, emerged as an important local context that influenced all small-scale changes and LST initiatives. Participants confirmed that the history of past endeavours enhanced or eroded trust and affected the quality of local relationships.

Sort of you're always working against the history or you're working with the history, one of the two. (workshop participant)

\section{Mechanisms}

Trust emerged as an important mechanism triggered by a history of working together. When local system partners trusted each other, they were more willing to come together, share resources, data and ideas, and attempt to make changes.

Ninety percent of survey participants either agreed or strongly agreed that trust among Alliance partners was influenced by the history of working together. It was the process of change and how it was done, for example 'bottom-up' rather than 'top-down', that developed trust, and not necessarily whether the attempt failed or succeeded. With high trust, failure of efforts in the past 
were seen as opportunities from which to learn and do things differently. Conversely, where there was mistrust, senior system leaders and frontline staff were less willing talk to each other and share their intelligence, let alone embark on change efforts. Participants observed that trust was enhanced when previous efforts were successful. Trust was eroded, or mistrust was created, when grievances from the past were embedded in folklore.

I used to call it folklore - in New Zealand, clinicians and boards could recount stories, which were about creating mistrust and dwelling on grievances of the past. (IP03)

According to participants, the presence of trust determined the quality of local relationships, especially between senior leaders of DHBs and PHOs. The quality of relationships was an intermediate outcome and was a pre-requisite enabling contextual factor for Alliances' being successful in their attempts to implement the SLM programme.

In the New Zealand health system, the key roles that determine the quality of local relationships at the district level are Chief Executives of DHBs and PHOs and General Managers of DHB Planning and Funding Units. The latter title varies across the country, but the role has the responsibility for planning and funding of all health services delivered in the district. Given that DHBs receive majority of health funding, and with contractual arrangements in place between DHBs and PHOs, these are powerful roles in the New Zealand health system. Nearly all survey participants (98\%) either agreed or strongly agreed that the nature of relationships of those in these roles had a significant influence on the implementation of the SLM programme.

One of the participants said that "transformation is about people and relationships" (IP06). A survey participant expressed that they were "lucky to have a CE who values relationship building". Other participants also highlighted similar sentiments.

We think that a great deal of the change that we need to make in the health system is dependent on having really strong collaborative relationships within and across organisations. (IP08)

In a complex adaptive system, most contexts such as the demographic profile of a district, and the fiscal and political environments, are outside the system leaders' control. Relationships are one of the few aspects that system leaders can influence and use to influence other contexts (IP06). A survey participant referred to strong relationships as a "fundamental building block". 
One survey participant said that their historical experience with the MoH negatively affected the time and value their senior leaders placed on the SLM programme. Another (IP06) said that history affected culture and practice, while the long legacies from how health and social care professionals have worked historically makes it difficult to change the culture; "so it's about learning to trust each other and to work in harmonious collaboration" (IP06). Participants reported that high-trust relationships take years to develop and sustained trust efforts are needed to nurture and sustain these.

Participants agreed that when local relationships were of high trust, it was much easier for DHB senior leaders to practice a distributed style of leadership in the Alliance. Where trust was missing or where mistrust was present because of negative history of working together, the relationship between senior leaders of DHBs and PHOs was one of 'master-servant'.

Participants said that trust between senior operational and clinical leaders was crucial to the success of any LST initiative. Trust was gained with clinical leaders when they were given the tools, were involved in the design of solutions and saw their solutions being implemented. This empowered clinicians to improve the system they work in (IP02). Top-down approaches were seen as compliance exercises and led to health care professionals being cynical of or disengaging from future LST initiatives.

The key mechanisms triggered by a history of working together that influence the successful implementation of LST initiatives and the outcomes that follow are summarised in Figure 11.1.

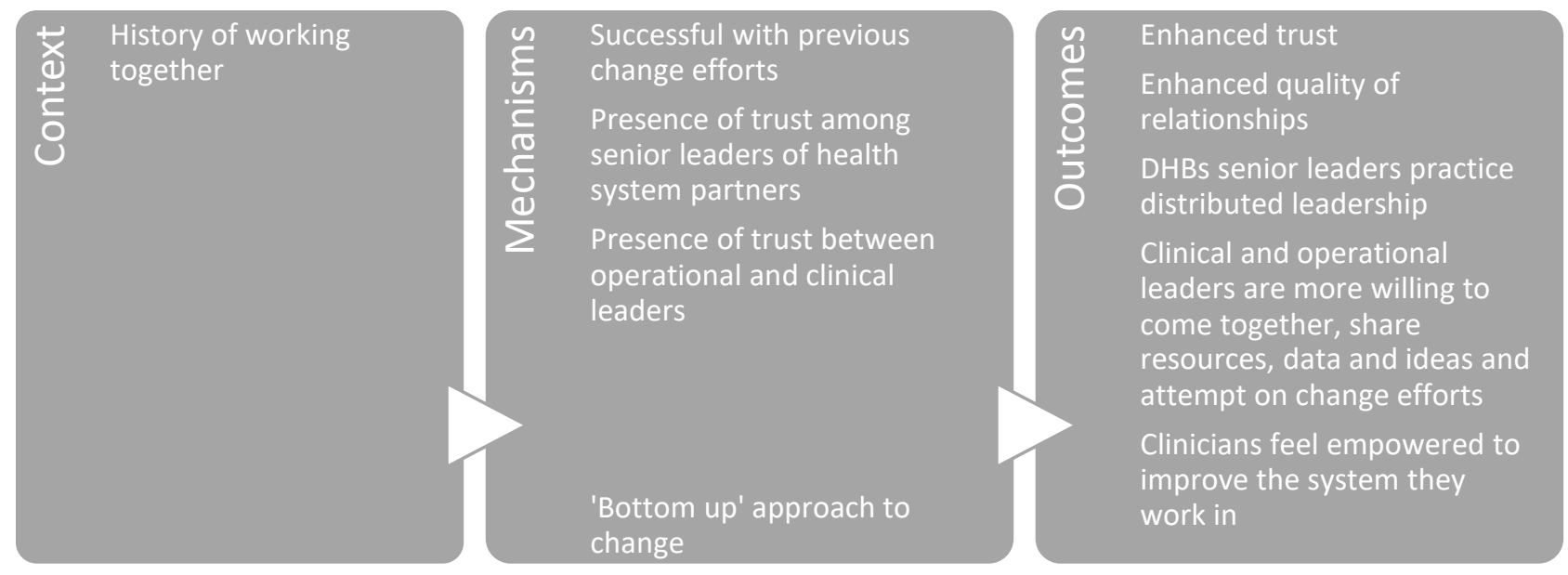




\subsubsection{Distributed leadership from DHBs}

While the distributed leadership style from DHBs was an intermediate outcome produced by a history of working together $(C)$ in the presence of trust among senior leaders $(M)$, participants also identified distributed leadership as a key local context. Participants said that transformative change needs a leadership that is much more collaborative, and leaders that are not organisationally focused, but are system- and outcomes-focused. This nurtures partnership and high-trust relationships and people do not feel threatened by the size of the organisation (such as the size of DHBs or PHOs) or funding (IP09).

Participants said that Alliances provided a key policy mechanism through which DHBs demonstrated a distributed leadership style.

Many workshop participants debated that the distributed leadership should extend beyond the health system to social care partners such as the Ministry of Social Development and the Accident Compensation Corporation. However, in the end they agreed that this was dependent of the maturity of local Alliances and local relationships and that there was much to improve within the health system first.

\section{Mechanisms}

The explanation for how distributed leadership works emerged from research participants who said that people feel less threatened with distributed leadership as they see evidence of power being shared equally and this has a ripple effect of building trust and commitment among Alliance partners

All workshop participants unanimously agreed that distributed leadership sustains and nurtures trust and commitment among health system providers in the district because it provides an opportunity for equal power at the decision-making table rather than the power sitting with the larger organisations because of their size or the funding they bring to the table. Eighty percent of survey participants either agreed or strongly agreed that distributed leadership built commitment among the Alliance partners.

A distributed leadership style led to a collective approach to the implementation of LST initiatives that is 'best for the system and best for the patient'. This was supported by survey participants who said:

Building trusted equitable relationships across the local health environment and using a collective impact approach with participants so that all parties have equal 
power at the decision-making table, rather than the party with the funding. (survey participant)

True partnership rather than a DHB big brother approach dominating - trust in PHOs and trust in clinicians. (survey participant)

The quality of relationships built by presence of trust extended beyond DHBs and PHOs, which are the two largest providers in the health system, to other small community-based health services providers, and most importantly to local communities and the Iwi. One interview participant (IP06) said that where there was deep rooted mistrust, no matter how much effort or money you threw at it, LST initiatives would not succeed.

Participants from the workshop and the survey said that, as the organisation that receives the majority of the health funding and the inherent power that comes with it, trust must be given first by senior leaders of DHBs to other providers in the health system. This action sets the tone for relationships in the district; whether it is one of 'master-servant' or of equal partners.

The DHB has to trust. It can't sit there and say, well, I will trust you when you behave in a trustworthy way. It actually has to say, okay, we're going to trust the system, and then work with it. That one action will change a whole heap of dynamics. (workshop participant)

The non-health interview participant (IP10) emphasised the importance of trust in relationships to rely on people in the system to do their jobs. One of the workshop participants who was a senior leader from a DHB described their deliberate strategy to build their local health system based on trust through distributed leadership.

We deliberately set out to build a system based on trust. It was a deliberate action. It wasn't by a chance. It was actually a strategy to build a system based on trust. (senior DHB leader - workshop participant)

The distributed leadership style from DHB senior leaders to an Alliance fostered commitment from senior leaders (both operational and clinical) to take a collective approach to the implementation of LST initiatives. This meant working beyond their organisational and professional boundaries towards a shared vision and goals with a sustained commitment, even though the results of LST initiatives cannot be seen in the short-term. Participant IP02 described commitment, from their experience as the chair of an Alliance leadership team, as: 
...working beyond and throwing the boundaries of the organisation that you work for and are employed by, such as a DHB or a PHO when going into the room, or throwing away the boundaries of the particular area of clinical practice that you happen to hail from, and going into to work in the Alliance with the whole of system approach, essentially. (IP02)

One participant (IP08) highlighted that often system leaders try to draw boundaries in the system; to wrap their arms around it to control the system. This inherently does not work in a complex adaptive system where the boundaries are blurred, and a collective approach allows system leaders to understand the different players and factors, and dynamics between them, which leads to system leaders "guiding the system and not controlling the system" (IP08).

Participants stated that strong and courageous leadership was required to demonstrate commitment, which had the power to change the priorities and perceptions of those in the system. When staff were clear on the vision and goals they were trying to achieve, they used these to prioritise their work and felt confident to do things differently and work beyond their organisational and professional boundaries to achieve the vision and goals (IP06, IP07, IP09 and workshop participant).

In theory, evidence and data will drive change - if people see the issues and needs clearly identified, then they will do the right thing. In practice, there is lots of patch protection and managers don't show enough bravery or leadership to break down barriers across organisations. (survey participant)

We just need to have the spine and the courage and just keep going and not bottle it and revert back into the old ways. (IP06)

Through the use of a distributed leadership model, system leaders were able to develop and agree on a shared vision and goals. The shared vision and goals gave system leaders a collective burning platform for transformational change and to collectively attempt to resolve financial pressures, understand the national policy and political contexts, and improve health service delivery rather than individually or within their own professional discipline. Resources for LST initiatives, such as the SLM programme, were able to be prioritised and aligned to achieve the shared vision and goals.

A vision for the district required DHBs to recognise the system and actors outside the hospital and focus on the contribution of the whole health system to the shared vision and goals. Several 
survey participants reported that a desire to retain power and control by senior DHB and PHO leaders and a focus on the hospital by DHB senior leaders hindered the progress of the SLM programme.

Person-specific desire to retain power and control by one key partner. Very slow to start with and not accelerated until change in DHB leadership. (survey participant)

Participants also highlighted that the complex adaptive nature and history of changes in the health system can cause change fatigue and a resistance to change (IP08 and workshop participant). An agreed shared vision and goals that are clearly communicated by senior system leaders and that capture the hearts and minds of actors in the system is needed to overcome the inertia in the system, which is powerful in blocking LST initiatives.

You have to have focused leadership. You need to communicate the end goal, what you're actually trying to achieve. It's not always just about the project. And then work that and communicate that to your team. Just having that clarity of communication, which is absolutely vital. (IP10: non-health interview participant)

The key mechanisms triggered by a distributed leadership from DHBs that influences successful implementation of LST initiatives and the outcome that follows are summarised in Figure 11.2.

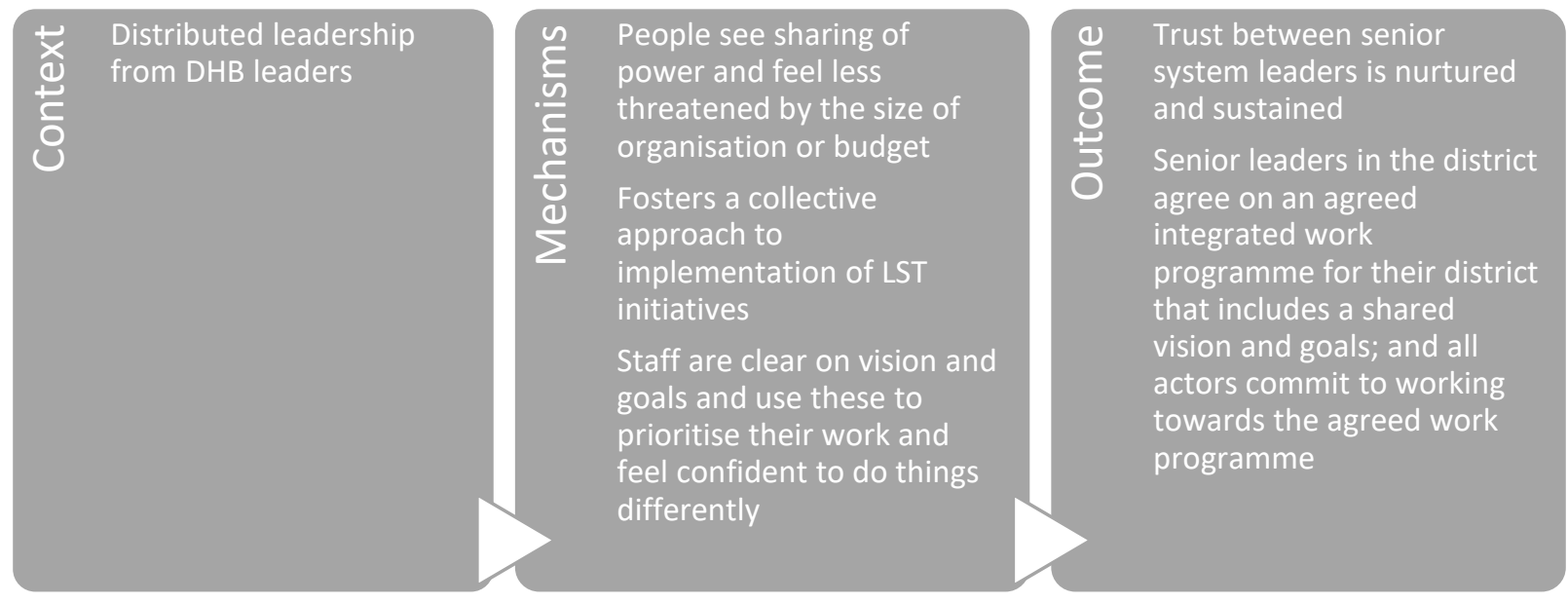

Figure 11.2: CMO theory - Distributed leadership from DHB leaders 


\subsubsection{Maturity of Alliances}

Participants said that Alliances were an "extremely powerful mechanism" (IP02) for bringing key system partners round the table to deliver co-ordinated and integrated health care and one that is responsive to health needs of the local population. Although Alliances were introduced as a policy mechanism, the alliancing way of working depended on the interactions and relationships of Alliance partners, which drove their reasoning and behaviour, which in turn influenced the success of Alliances. Participants identified that this reasoning and behaviour contributed to the maturity of Alliances, which influenced implementation of LST initiatives.

A survey participant identified that the lack of an Alliance in their district hindered the implementation of the SLM programme. Another said that individual personalities, lack of agreed governance, and unclear leadership and accountability affected the success of the SLM programme in their district. Both participants said that this resulted in a lack of leadership and joint decision making.

It made the process difficult in terms of identifying who the right people around the planning table should be. (survey participant)

According to participants, mature Alliances have common features of: broad membership that brings perspectives from the whole system including communities and Iwi; clearly defined roles of Alliance members; a clear decision-making framework; an independent chair; dedicated resources; and a clear mandate and governance structure for the Alliance. However, one of the participants who works as a consultant with DHBs to integrate hospital and primary care services (IP07) said that maturity required more than the structure and key processes and relied on relationships and working as a community towards a shared vision. These features were also identified by workshop participants in the maturity matrix for an alliancing way of working.

Another participant who had experience as a chair of an Alliance (IP02) said that maturity of an Alliance relied on all members of the Alliance committing to the principles of the Alliance charter, as well as a distributed leadership model, a positive history of working together, high-trust relationships between senior leaders of DHBs and PHOs, a commitment to delivering a patientcentred health service and a commitment to meeting obligations of Te Tiriti o Waitangi.

... if you don't have those key people there at each meeting and absolutely committed to discussions, it becomes extremely frustrating. (IP02) 
Participants said that less mature Alliances lacked a whole-of-system approach; sharing of data and intelligence; and a shared vision and goals. This led to each provider working to their own priorities or agenda.

\section{Mechanisms}

According to research participants, mature Alliances did not trigger the mechanisms of high trust, shared vision and goals and commitment. Instead, these are pre-requisite enabling contextual factors for an alliancing way of working. In the presence of these enabling factors, Alliances are more cohesive and resilient, which helps them navigate through disagreements, deal with emerging issues, overcome changes in senior leadership roles and Alliance membership, and stay focused to the shared vision (IP09).

Survey participants said that districts with mature Alliances were more successful with the implementation of the SLM programme as they had established relationships and Alliance members were used to taking a system-wide view. They were able to set up lower level structures in the form of steering groups and champions for each system level measure and Alliance members took ownership of their role in meeting improvement milestones for the measures. There was an overall buy-in to the SLM plan and the measures. This buy-in made the programme more sustainable in their districts.

We also set up a steering group for the development of the SLM programme and identified champions and leads for each. Each organisation took ownership of its role with meeting the measure. (survey participant)

The key mechanism triggered by maturity of Alliances that influences successful implementation of LST initiatives and the outcome that follows are summarised in Figure 11.3.

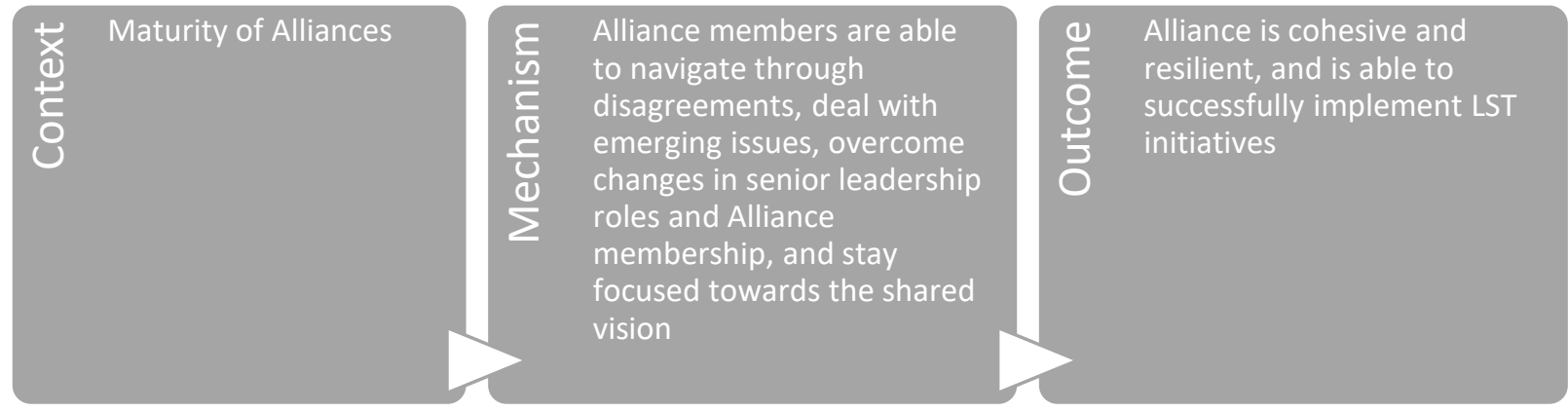




\subsubsection{Capacity and capability}

Capacity and capability emerged as important contexts for implementation of LST initiatives and participants identified two key elements critical to these contexts: integrated health information and analytic capability (as discussed earlier in Chapter 9). The presence of these elements enabled those in the system to turn data into knowledge, identify gaps, test new solutions, promote success and develop action plans to address health inequities. Two survey participants said there was a lack of capacity and capability at the national level to support system-wide change. There was difficulty in getting timely national level data and some of the data is not consistently captured.

Participant IP07, who worked with many DHBs as a consultant, used the sliding puzzle analogy to describe capacity: where one free space is needed to move tiles along to get them in the order. Tiles in the puzzle cannot be moved around without the free space. Similarly, DHBs and PHOs must create resources that can float around and across organisations and upskill these people in change management. Another participant said that "there has to be specific allocation of the necessary resources to innovate, to do things differently. Usually people are forced to try to do this from the corner of their desk and it doesn't work" (IP08). A few survey participants identified the lack of dedicated analytic resources, skilled project or programme managers, budget, and evaluative processes as important contexts that influenced implementation of the SLM programme in their district.

Participants said that sometimes capacity is about freeing up frontline health care staff so they can participate in LST initiatives. One of the interview participants (IP02) and a workshop participant highlighted that for privately employed clinicians, this includes reimbursing them for their loss of income and costs such as backfilling with a locum and travel to meetings; it also meant having short meetings and at times suitable for clinicians to attend, for example in the evenings rather than during clinic hours. A survey participant said that the time available to engage collectively by senior leaders and managers in the identification of improvement activities in the SLM plan was limited. This led to activities being proposed by those facilitating the development of the plan rather than being developed by clinical leaders following discussion with frontline workers. This may have resulted in less ambitious actions than would have been chosen otherwise. 
One survey participant highlighted that the capacity and capability of DHBs vary and that smaller DHBs lack sufficient capacity and capability to implement LST initiatives. This is exacerbated by a lack of awareness by some PHOs and DHBs about how limited their capacity is (IP01).

System leadership capacity, capability and consistency at political, policy and delivery levels were identified as important contextual factors for implementation of LST initiatives. System leadership capacity and capability facilitate "sharing of information to think about the priorities for that subregion, region or national than local" (IP03). It also helps overcome historical grievances such as organisational reforms involving 'top-down' attempts to merge DHBs, DHB planning and funding units or health services and supports and mentors clinical and operational leaders to bring the "relational capital" (IP03) that is so critically important back into the system.

We need to ensure that we're supporting those leaders and setting the right expectations around what it means to be a leader of the system. (IP03)

Participants said that continuity of leadership in the time scale that is needed to create the circumstances and conditions for transformational change was difficult in the health system. Frequent changes to leadership disrupted LST initiatives through changes in policy and direction. The interview participant from Scotland talked about disruption to LST initiatives caused by changes in legislation.

I think it's very difficult to nurture transformational change in a system where you've got very frequent changes of administration with a 360 or 180 degree turn in some of the directions and some of the legislation, and some of the levers you can pull. (IP06 - interview participant from Scotland)

A lack of system leadership capacity and capability stops the health system from growing or advancing as a system and impacts on the performance lift needed or desired (IP03).

Some participants from New Zealand and overseas (IP06, IP07, IP08 and IP09) suggested that in rural and remote settings, where health services are delivered by a small number of providers with limited capacity and capability, more effort is made to build high trust relationships so that different health system providers can work together effectively to deliver high quality health care to the population.

I think in a lot of our rural and remote areas, although they have their own challenges because they don't have the same access to services, which is in itself 
another issue, but they also have quite strong relationships because the Royal Flying Doctor Service is the only service coming in and they don't have a doctor but they have a paramedic and they have a nurse. You automatically have to work as a team even though you're covering an area this big. (IP09 - interview participant from Australia)

Participants said that the culture in rural areas was different to that in urban areas. There is a stronger sense of community in rural areas and health system staff work beyond their organisational boundaries to deliver a patient-centred service, as a New Zealand participant said:

If you work rurally, everybody seems to go the extra mile, they all have a common purpose which is to keep those people well and they go beyond their normal existing boundaries. (IP07)

Conversely, in urban settings, where there was more capacity and capability, the need or vigour is not there to invest in long-term relationships or to consider the disruption to patients' lives as they live closer to hospitals and have better access to ambulance services (IP07). Overseas participants in particular who had led transformational change in health systems suggested that urban health services could learn from remote health services when implementing LST initiatives.

What we find in British Columbia, and I'm guessing it might be true in New Zealand as well is that there are very different cultures in rural context than in urban context and it's much easier to get the kind of collaboration that's necessary, get all the key players around the table in the more rural settings, so that in the urban settings the critical thing is to look at the structural and the political context and try to understand how that's either facilitating or getting in the way of transformative change. (IP08 - interview participant from Canada)

\section{Mechanisms}

My initial proposition was that when the system leaders recognise the importance of capacity and capability needed for implementation of LST initiatives, they were more likely to invest in it. Participants confirmed this proposition to be true. Eighty-six percent of survey participants either agreed or strongly agreed that senior leaders were more likely to invest in capacity and capability if they understood and valued the use of data and evidence. Participants said that the recognition of and investment in capacity and capability by the system leaders contributed to the system's readiness for change (IP05 and IP08). 
Workshop and interview participants provided richer explanations of how capacity and capability influenced the successful implementation of LST initiatives. Analytic capacity and capability enabled a deeper understanding of variations in health care provided, which empowered clinicians to engage in system improvement and system leaders to think beyond their unit, department or organisation and more about the whole system.

Close to $73 \%$ of survey participants either agreed or strongly agreed that the DHB analytic capability enabled Alliance teams to better understand the variations in health care provided to their population and address health inequities for Māori and other high priority populations.

Eighty-eight percent of survey participants either agreed or strongly agreed that use of data and evidence led to stronger clinical engagement in the identification and implementation of quality improvement activities at the district level. Further, $90 \%$ of survey participants either agreed or strongly agreed that when health care professionals saw the value of integrated health information, both for themselves and their patients, they were more likely to share patient level data across the system. The capacity and capability for improvement empowers clinicians and brings them to the table to improve the system (IP02 and IP06). Participant IP06 talked about the 'power of data' to bring clinicians together and using data to enable reflective practice, including patient and carer feedback and experience data: "the power of stories - that really brings clinicians to the table" (IP06). The analytic capability enables transformation teams to ask the right questions and use data both as a 'rear-view mirror' and to predict future demands.

One participant (IP03) talked about the effect of system leadership capacity and capability that enables leaders (both clinical and operational) to think beyond their own unit, department or organisation and more about the system and which is currently lacking in the New Zealand health system. With the vast amount of health data available, a lack of system leadership can overwhelm DHBs on how to use this information to ensure that LST initiatives are contributing to the shared vision and goals (IP05). System leadership capacity and capability enables DHBs to identify and share the analytic capacity and capability needed to achieve the shared vision and goals.

Participants said that constant organisational reforms added to change fatigue and inertia in the system and identified these as constraining and disrupting LST initiatives. One of the consequences of constant organisational reforms is a high turnover of staff, in particular senior and middle operational leaders who often championed LST initiatives. The loss of institutional knowledge and disruption diminishes the system leadership capacity and capability. 
The key mechanisms triggered by capacity and capability that influence successful implementation of LST initiatives and the outcomes that follow are summarised in Figure 11.4.

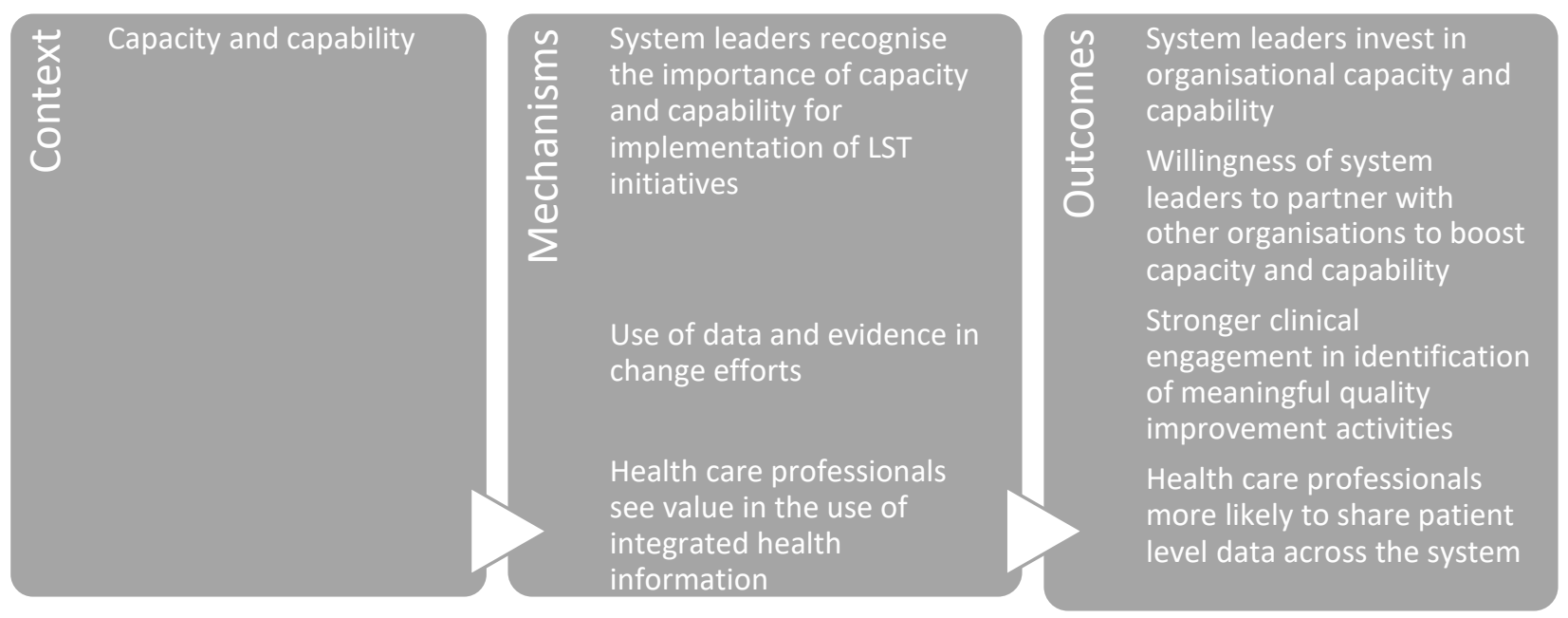

Figure 11.4: CMO theory - Capacity and capability

\subsubsection{Continuous improvement culture}

Participants agreed with my initial proposition that a continuous improvement culture was an important contextual factor for implementation of LST initiatives. Participants expressed the view that a continuous improvement culture enables continuous learning; people being curious about the status quo and motivated to test and challenge it, as IP03 said "a thirst for open critique and challenge". This continuous improvement culture is enabled by people having the resources and skill to understand what good looks like, how they can assess their maturity and what does the best in the industry or system look like? Another participant (IP04) said that the continuous improvement culture is signalled from the top of the organisation and is a sign of a mature system. Sometimes people are busy reporting on accountability systems and they do not have time or will to focus on quality improvement and even if they do, it is through "a tick box exercise" (IP04). An accountability focus usually has benchmarks or thresholds and these can often stifle continous improvement as this "has a great effect of making people be happy in the middle of the pack and not seek to improve" (IP04).

Participants identified that active effort and time is needed at all levels in the system to build a culture of continuous improvement.

You need to create - and it takes a lot of time and effort - over time a system where your leadership are all people who believe in quality improvement, professional collaboration in practice. (IP02) 
One participant (IP08) said that creating population level change takes several years and it is difficult to show short-term impact and therefore, finding the right balance between accountability and continous improvement can be difficult. Instead, continuous evaluation or 'developmental evaluation' that is more fluid is needed for "revamping of the strategy as you go forward" (IP08). Others reinforced the importance of processes such as the plan-do-study-act cycle and constant feedback loops that are crucial for the successful implementation of LST initiatives.

\section{Mechanisms}

Feedback from participants emphasised that senior leaders' sponsorship influenced the time commitment and the local policy environment, which subsequently affected activities and outcomes. While small-scale change efforts can be supported by middle managers and frontline health care professionals, LST initiatives require recognition, support and visibility from senior system leaders. One participant said: "you have to have buy in at the very highest level" (IP08). Where senior leaders do not have an understanding and appreciation then they are likely to sabotage or underappreciate or fail to adequately to support the grassroots innovation that is critical to success (IP08). However, participants saw my initial proposition as an important enabling contextual factor and not a mechanism that is triggered in the continuous improvement culture context.

Instead participants expressed the view that culture of a 'permissive' environment from senior leaders of system, including the $\mathrm{MoH}$, made staff feel safe to try new initiatives in collaboration with other parts of the system and feel supported and encouraged without the worry of failure or blame. A 'permissive' environment is where senior system leaders actively support a continuous improvement approach.

Transformation works where you have a culture of continuous improvement. (IP12: non-health participant)

Rather than a pass or fail approach that goes with accountability, a continous improvement culture is about establishing "safety rails" (IP04) and allowing organisations to operate within those margins and providing them with support and mentoring that will build a learning system (IP03 and IP04).

Actually, giving permission for organisations to be open about the fact that they're not actually achieving as much as they want to and give them some support or 
give them some flexibility for change. Come back to that point about the safety rails. (IP04)

Furthermore, continuous improvement culture allowed organisations to embrace critique and look to improve their performance (IP03).

The key mechanisms triggered by a continuous improvement culture that influences successful implementation of LST initiatives and the outcome that follow are summarised in Figure 11.5.

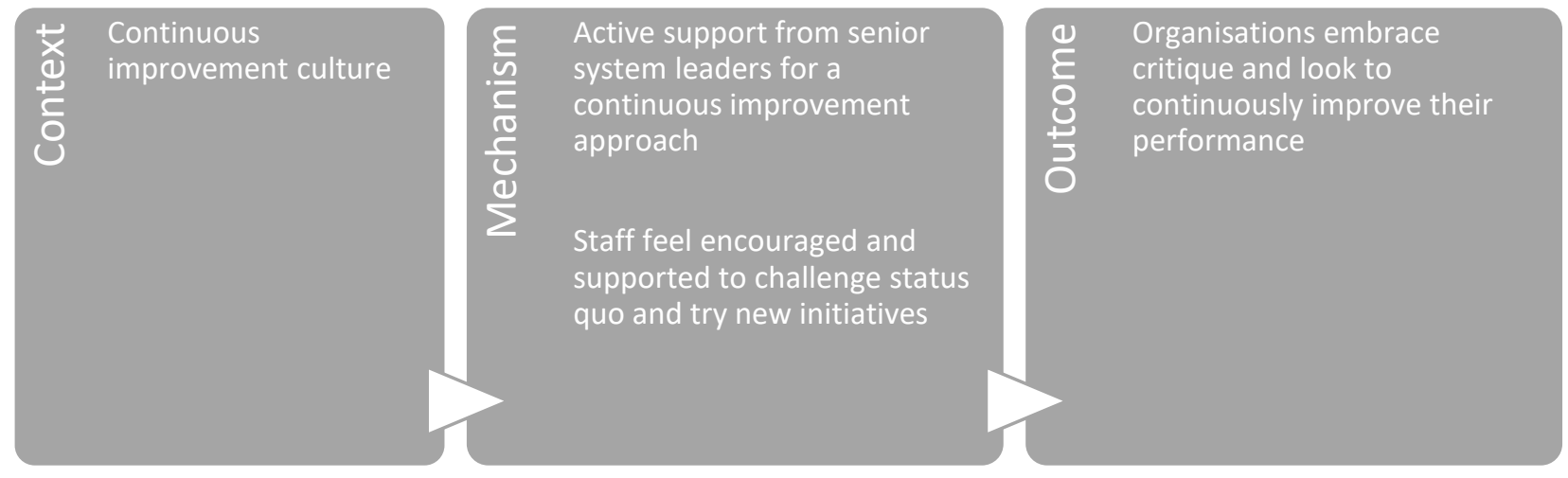

Figure 11.5: CMO theory - Continuous improvement culture 


\subsection{National contextual influences from the research}

This section discusses macro level contexts that influence a district's ability to successfully implement LST initiatives. I theorised that the use of 'new power' values of collaborative design with a continuous improvement approach and implementation through Alliances positively influenced implementation of the SLM programme because it enabled a buy-in from the sector, which made the programme sustainable.

In addition to my initial proposition, participants identified several additional contexts at the macro level that influenced successful implementation of LST initiatives. These are discussed in the next section.

\subsection{1 'New power' approach to design and implementation of the LST initiatives}

Participants provided further explanations on how the use of 'new power' approach positively influenced successful implementation of LST initiatives. Further, it revealed how the 'new power' and 'old power' approaches can work against each other to constrain successful implementation of LST initiatives.

A survey participant said that the co-design workshops for the SLM programme, governance, training, communications and financial incentives were enabling contextual factors for the programme.

Workshop participants cited the use of 'new power' values of time, commitment, and the collaborative way of working with the implementation of the SLM programme, which had created high-trust relationships, as a motivation to prioritise their participation in the research workshops.

The other reason I am here is because it's payback for both of you because you stuck at it, you enabled it, you made it work, you enabled us to do it the right way, which frequently doesn't happen with Ministry-led projects. But you enabled us to actually build this programme in the right way. (Senior DHB leader at the workshop)

A few survey participants said that the 'new power' approach created a disconnect between the SLM programme and the district annual planning processes, which used the traditional 'old power' approach. This created confusion at the district level as to how the SLM programme fitted in with other national strategic and operational plans and the continued need for multiple planning documents. One survey participant said this led to a disintegrated approach and less 
buy-in at the senior leadership level in their district. Others said it led to a lack of visibility, a lack of a shared understanding of the purpose of the SLM programme, a lack of integration into the DHB annual planning processes and a lack of credibility for the SLM programme.

\section{Mechanisms}

The explanations from participants highlighted the tension created in the system with the use of both 'new' and 'old' powers. The tension was created by a lack of understanding about system accountability and performance improvement by senior system leaders and their leadership style to respond to these two demands of the system.

Participants said that the 'new power' approach with the design and implementation of the SLM programme increased engagement from Alliances as there was a recognition that the programme was new and that districts would not always get the expected results: "we were trying a new approach and would not always get it right or get the results we expected" (survey participant). The 'new power' approach generated buy-in from senior system leaders and implementers, which builds confidence in them to be innovative and do things differently, leading to sustained engagement with implementation of LST initiatives. In saying that, demands of system accountability that predominantly uses an 'old power' approach can undermine 'new power' values of distributed leadership and shared commitment towards common goals.

The key mechanism triggered by a 'new power' approach to design and implementation of LST initiatives and the outcome that follows are summarised in Figure 11.6.

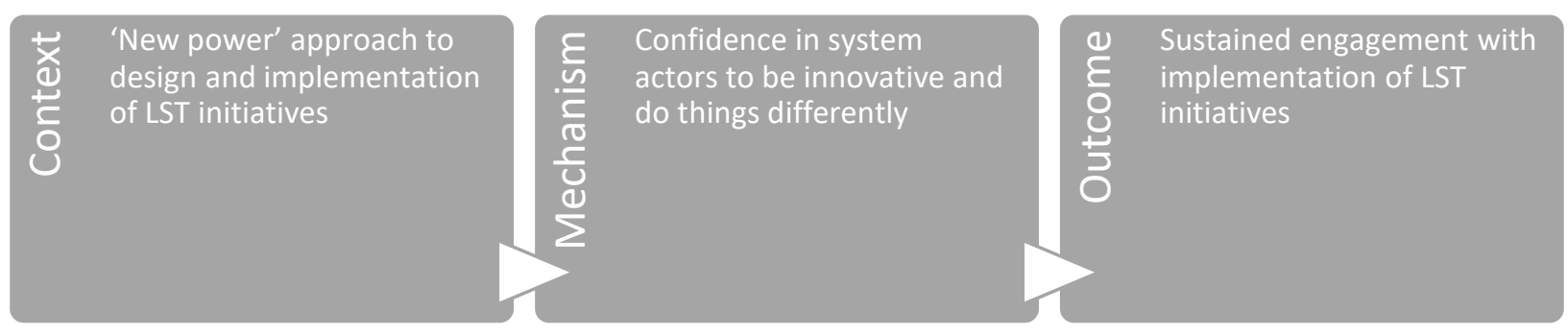

Figure 11.6: CMO theory - 'New power' approach to design and implementation of LST initiatives

\subsubsection{Political environment}

Research participants identified three contexts in the national political environment that influenced successful implementation of LST initiatives: the election cycle, national policy environment, and government ideology. These contexts influenced the political culture in which Ministers and the senior leadership teams of the MoH and DHBs work in. 
One interview participant (IP08) said that the Minister of Health is an elected official and not a professional position and therefore is driven by the culture of representative democracy rather than innovation and transformation. This means that they may not be engaged or supportive of LST initiatives that cannot show short-term improvements. Therefore, participants believed that it is important to create leadership at the highest level so there is a distinct leader who is responsible for making the change and who can work in parallel with those who are responsible for implementing the change. This person needs to be reporting to the Minister of Health or similar with a degree of independence and the freedom to really be innovative and to do things differently (IP08). Secondly, it is important to have a forum such as a high-level think tank that can link different levels of policy from the executive to the frontline to facilitate national policy leadership to create a shared vision for the system and provide thought leadership on understanding the context before initiating transformative change. The interview participant from Canada warned against the short-term initiatives and referred to pilot studies as "the kiss of death for transformative change" (IP08).

\section{Mechanisms}

Participants said that New Zealand's three-year election cycle means that there is a short-term focus that mirrors the election cycle, which causes rapid change in national policies and budget packages. Participants (IP04, IP12 and workshop participant) said that this rapid change creates instability in the national policy environment as national policies follow the election cycle and the incoming government sets the performance and improvement agenda. When a government is elected, they spend the first year to come up to speed and take a steady approach in the third year in the lead up to the next election. This leaves the second year, or commonly referred to the 'year of the delivery', when most change happens with typically in health and education that are two of the key areas with most reforms (IP04). The short-term focus hinders the health system from having a long-term planning strategy and commitment and LST initiatives are disrupted.

Two interview participants (IP08 and IP10) talked about the importance of having a long-term planning strategy that is more than 20 years, with a focus on how the system might keep up with and develop to ensure it can meet increasing capacity demands.

The interview participant from the construction industry (IP10) said taking into consideration the rate of capacity demand for hospital services is important when constructing new hospitals. A 10 -year horizon means that by the time the hospital build is finished, there are only about three 
years left to use that hospital before it reaches that 10-year capacity and then plans for expansion starts again. A 20-30-year view would allow the hospital to keep up with the capacity demand.

One interview participant (IP06) mentioned another effect of short-term focus is the change in personnel holding key leadership roles in the health system, such as the Director General of Health, the DHB Board Chairs and the Chief Executives, all of which have an impact on the system's leadership, direction and priorities. Workshop participants talked about the change fatigue that comes with constant change in the national policy environment and the key national leadership roles. This constant change often leads to additional priorities or projects to deliver on. Participants said in the current accountability environment, the MoH makes it extraordinarily difficult to stop work programmes. A survey participant said that an overload from other areas means less time for the "good stuff like the SLMs".

We've got all this stuff already and now this is another thing to do and we never turn anything off. (workshop participant)

The constant change also creates resistance to change or inertia in the system.

When you're dealing with a large system that's been in place for a long time it's very hard to get people to want to change. (IP08)

One of the non-health interview participants (IP12) talked about health system lacking a clear strong foundation with key public health objectives that are based on evidence, for example, immunisation rates, water fluoridation, information management principles and standards of clinical practice. If the health system had agreed key objectives for the system, informed through an appropriate governance model, then it becomes harder for the incoming Minister of Health to challenge these and disrupt LST initiatives underway in the system. This will enable the system to have a long-term focus that is enduring to change in governments. The workshop participants supported this view and discussed ways of divorcing the health system stewardship from the "whims of ministers or the colour of the party", such as the upcoming reform from the Health and Disability System Review commissioned by the government. They went on to say that the senior leadership at the $\mathrm{MoH}$ had an important role with the advice that is provided to the incoming Minister of Health. They emphasised the need for better transparency about the advice provided to Ministers and their response to it. This transparency creates trust between the MoH and parts of the health system, especially with DHBs. 
The workshop participants and an interview participant (IP07) said the collaborative or competitive behaviour that follows the government of the day inadvertently sets the tone on how funders and providers behave, and whether they collaborate or compete for resources available. This significantly influences the alliancing way of working, which deliberately intends to remove competitive behaviours and nurture a collaborative way of working. It also affects the alignment of policies and agendas with other government agencies such as The Treasury, the Commerce Commission, the Ministry of Social Development, Oranga Tamariki and the Ministry of Business, Innovation and Employment. These organisations have different philosophical underpinnings to their policies and agendas but significantly influence health policies and the way health services are designed and delivered. For example, a couple of workshop participants discussed their experience with the Commerce Commission, which sets procurement rules that are based on patients having a choice in seeking health services. This leads to competitive behaviours among small health providers as they compete for contracts rather than working collaboratively to share resources and deliver what is best for the patient. This also has an impact on how health services are structured and procured by DHBs, who, while following procurement rules, also strive to offer high-value health care for their population and sustaining financial viability of their small community providers.

There were three contexts identified in the political environment that influenced successful implementation of LST initiatives. The key mechanisms triggered by these contexts: the election cycle, the national policy leadership and government ideology, and the mechanisms triggered and the outcomes that follow are summarised in Figure 11.7.

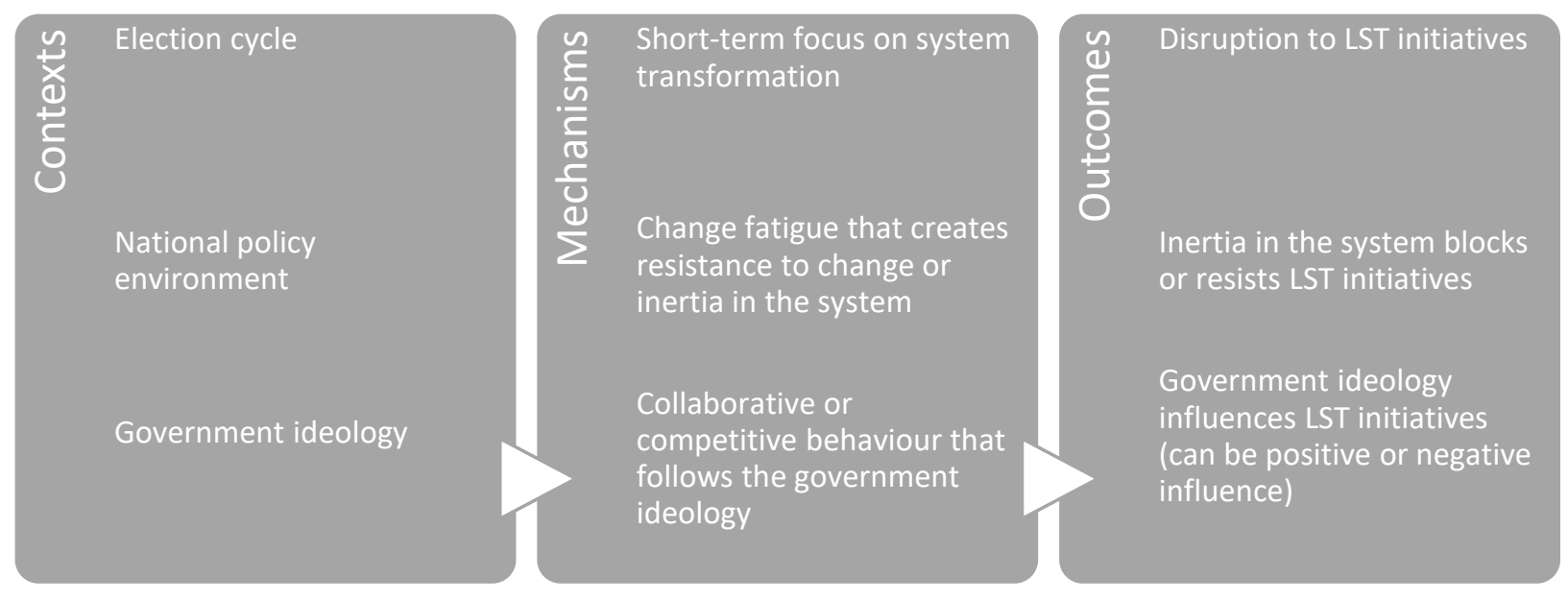




\subsubsection{Media bias}

Workshop participants identified media bias as a national context that influenced system leadership culture and political priorities. They discussed the influence of media, which reports on "sexy stuff that tends to happen in hospitals". This encourages the government and system leaders to use a transactional quality assurance approach where improvement has a direct attribution to an outcome or outcomes and can be reported in numbers and performance league tables. Participants discussed media reports when national health targets were being publicly reported and the impact of these. In some districts, this approach affected funding decisions where funding was directed to areas responsible for health targets at the expense of others. Participants also discussed the media being used by powerful lobby groups as a platform for their agendas, which then influences political priorities such as focus and funding for one part of the system, professional group, or a health condition. The establishment of the cancer control agency was cited as a recent example.

\section{Mechanisms}

Participants expressed the view that media influence fosters a blame culture where senior leaders are blamed and shamed in national media for failures in the system. This culture leads to a risk-averse approach by senior leaders where failure to meet national quantitative goals or measures is perceived as a failure of their leadership for which they are held accountable, with public reprimand or loss of their jobs. This approach suffocates the concept of a learning system and an aversion to LST initiatives that are not aligned with political priorities of the day and where results may not be visible in the short-term.

Media's underlying assumption is all about the transactional QA type of approach. If something goes wrong, it's somebody's fault and they need to be kicked, sacked. (Workshop Participant)

The key mechanism triggered by a media bias that influences successful implementation of LST initiatives and the outcome that follows are summarised in Figure 11.8.

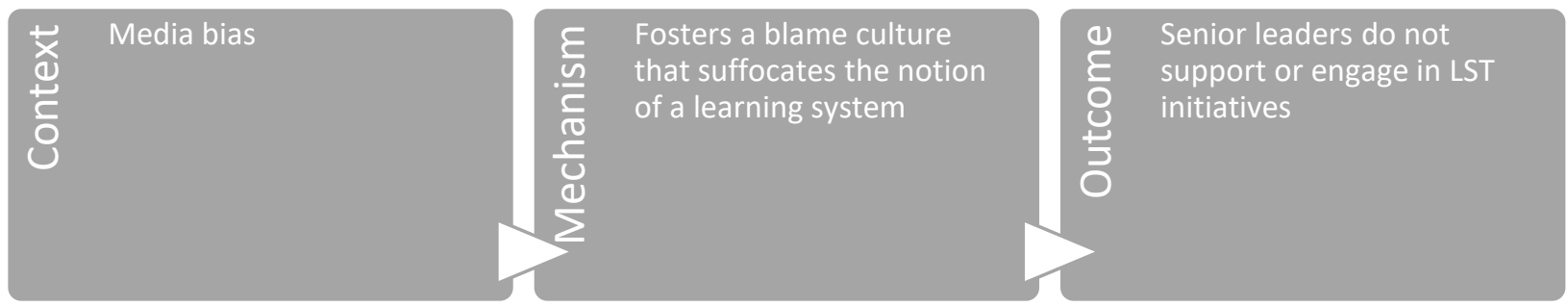




\subsubsection{Health system leadership}

The health system leadership was identified as an important and significant national context that influenced the successful implementation of LST initiatives. Some of this has been discussed above in relation to the political environment. Additionally, the $\mathrm{MoH}^{\prime} \mathrm{s}$ leadership style with system partners, in particular DHBs, determines the relationship between these two significant parts of the health system. Workshop participants said that a command and control style erodes trust while a collaborative or distributed leadership style builds trust and relationships.

\section{Mechanisms}

Participants stated that presence of a collaborative leadership fosters collective leadership from across the health system that enables the $\mathrm{MoH}$ to work with other senior leaders of the system to determine the shared vision or communicate the vision of the government. A shared vision helps to set consistent performance expectations for the whole system, which includes how the $\mathrm{MoH}$ monitors performance of the system beyond the DHB bottom line (overall financial position). A lack of shared vision drives the many organisations in the system to work to their own agenda or priorities. Fostering collective leadership is an important mechanism to support successful implementation of LST initiatives through facilitation, harnessing and sharing of capacity and capability, and supporting the $\mathrm{MoH}$ to incentivise improvement efforts. This also builds system leadership capability and facilitates collaborative learning. With strong collective leadership, the system has a better chance of managing emerging issues and uncertainty in the system and building a strong foundation that can influence political priorities and the media (IP12). System leadership capacity and capability also provides a mechanism to support the notion of a learning system (IP03).

Several participants (IP03 and IP06) talked about the variable capacity and capability in leadership across the system and the need for deliberate investment in the system leadership capability. This includes the $\mathrm{MoH}$ embracing the improvement function, setting the right expectations around what it means to be a system leader, and then supporting those leaders. Having PHO Chief Executives alongside DHB Chief Executives would break down some of the barriers and provide an opportunity for local systems to learn together and create 'communities of interest' that share knowledge and offer peer support and mentoring. Participants said that these 'communities of interest' cannot be left to happen organically and need careful management because if not done well, there is a risk that the attempt to create these communities will be perceived as a command and control from the centre. The interview 
participant from Scotland described their experience of setting up a joint improvement team that brought together people with different sectorial expertise who understood the complexities of leading LST initiatives. The joint improvement team became a learning collaborative for their LST initiative.

One interview participant (IP03) emphasised the importance of supporting and celebrating innovation, which supports the culture of continuous improvement and creates the conditions for collaborative learning among agents in the health system. The participant said this leads to building leadership capability in the system and breaking barriers between senior leaders of health providers, for example between DHB and PHO Chief Executives. It also gives 'permission' for health providers to invest, support and celebrate innovations and LST initiatives in their districts and share with their peers and colleagues across the system. The participant talked about the national health innovation awards that celebrated local innovations from across New Zealand, which brought people together to share their learning and supported the notion of a learning system. Another interview participant (IP07) discussed this at length and said that service improvements are often seen as "woolly and soft" and a nice to have "when the DHB bottom line is sorted". They went on to discuss the unique role of the $\mathrm{MoH}$, similar to that of DHB planning and funding units, which is information rich but not active in using the information to support system improvements.

Participants said that the absence of a national improvement forum deprives professional development of key leaders in the system and impedes collaborative learning. One overseas interview participant (IP08) strongly recommended that New Zealand, as "a necessary first step", created a leadership programme specific for health system leaders.

The key mechanisms triggered by the health system leadership that influence successful implementation of LST initiatives and the outcomes that follow are summarised in Figure 11.9.

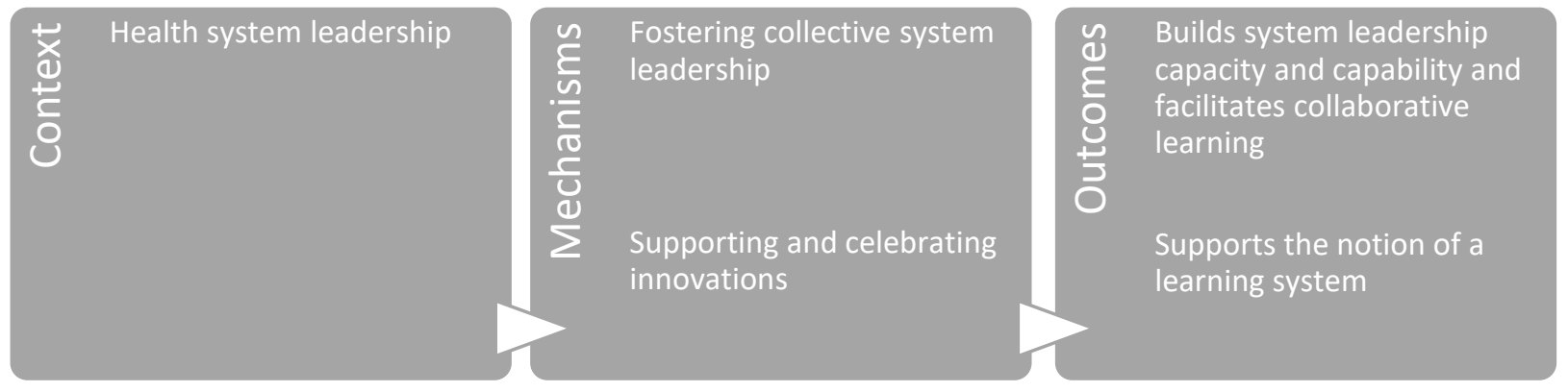




\subsubsection{Funding environment}

Participants discussed the impact of the funding environment on the successful implementation of LST initiatives and that it presents constraints and opportunities. The funding environment is an important context for the health system as most of the health care system is funded from general taxation.

Participants had conflicting views about the use of incentive payments as a policy mechanism for implementation of LST initiatives. Participants reported that the incentive funding for PHOs in the SLM programme created tension and mistrust between DHBs and PHOs. A couple of participants were critical of the financial incentive available to the PHOs when the governance responsibilities were held at DHBs. Further, there was a lack of transparency on how PHOs used the incentive funding to benefit the programme. One survey participant suggested that the funding needed to be placed with DHBs to be used as seed funding for innovative projects focused on improving equity for Māori. The survey participants said that the PHO incentives meant that some DHBs did not own the SLM programme or see it as a partnership. Instead these DHBs saw the SLM programme as a primary care performance programme and continued to focus on the hospital and its deficit rather than the system.

The participant from Scotland (IP06) talked about their use of a nationally held change fund to support transformation initiatives at the local level. The change funding provided to the local health and care partnerships was complemented with improvement support from a joint improvement team. According to the participant, it was the combined model of funding and the improvement support that made their transformation programme successful.

One interview participant (IP04) said that the issue with funding is there can be unintended consequences. Some system leaders are very quick to try and implement funding-related changes and use funding as the main lever that they can pull to influence system transformation, only to find that through unintended consequences or through various other elements, that funding either is not taken up, or it is taken up and spent in the wrong way or not in the way it was intended to be spent. Funding is a key enabler, but it needs to evolve and work with the transformative change and it should not to be taken for granted that funding will always create a catalyst for change (IP04).

\section{Mechanisms}

Understanding the system financial and accountability contexts triggered important mechanisms for successful implementation of LST initiatives. 
Participant IP06 shared their experience where the challenging financial agenda coupled with increasing demand for health services was a powerful motivator for innovative thinking and for system leaders and staff to think 'outside the square' (IP06). The participant talked about the continuum of financial context: from a "time of plenty" where everyone is comfortable and there is no reason to change and status quo is maintained, to a really difficult financial situation where everyone protects their own budget, are defensive, micromanage and "count every penny", not change and "just stay on the bike". It's when it gets beyond the latter stage of "beyond fixable with efficiencies and performance micromanagement" that disruptive innovations start. Leaders and staff understand that doing the same more efficiently is not going to be enough, that something radical is required. This provides the burning platform for transformation to take root and brings the political and the system leadership together to create conditions for transformational change to happen.

Participants highlighted that sometimes natural disasters provided the burning platform for transformational change and provides the lever of "let's not waste this opportunity for a kind of almost step change in practice or behaviour" (IP06). Events such as an earthquake or a storm blowing the roof off a hospital can be great catalysts for changes, such as shifting services away from hospital to community or people's homes, reducing the size of the hospital, the use of technology to provide health services, and more imaginative multi-skilled interdisciplinary teams working with health care professionals to the full scope of their roles.

The key mechanisms triggered by the funding environment that influences successful implementation of LST initiatives and the outcomes that follow are summarised in Figure 11.10.

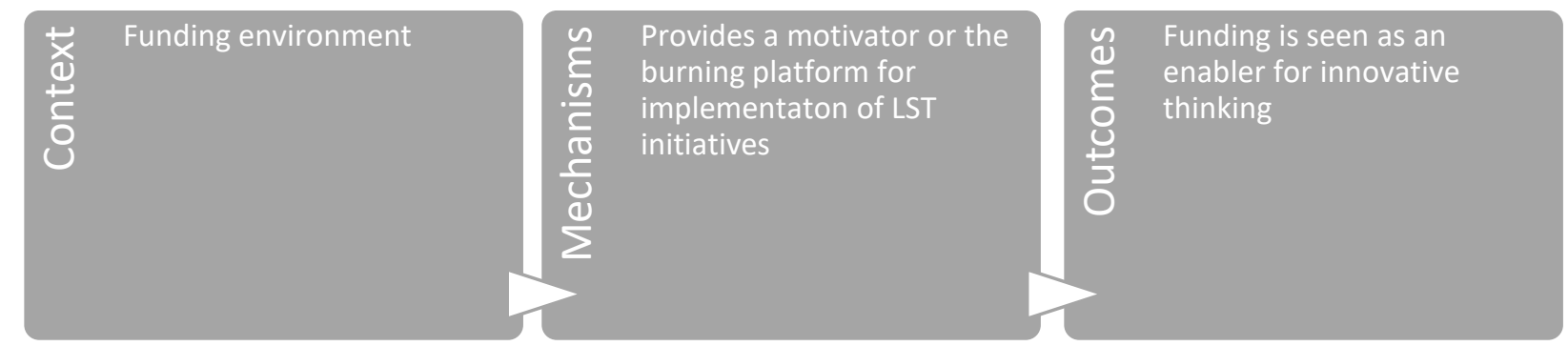

Figure 11.10: CMO theory-Funding environment 


\subsubsection{System accountability environment}

Participants were strong in their opinion that current system accountability arrangements, which focused on a small set of financial and performance measures significantly hindered implementation of LST initiatives in the health system because the focus of leaders remained on achieving this small set of measures. The workshop participants and the interview participants (IP02 and IP03) talked about the traditional financial monitoring frameworks that are national template driven, output based and focus on DHB financial position. This approach led to transactional relationships in the health system.

The current accountability arrangements have also led the system to be provider centric, rather than patient- and whānau-centric. DHBs focus their efforts on achieving the performance and financial targets and neglect their commitment to their communities and Te Tiriti.

\section{Mechanisms}

Participants highlighted two ways in which the system accountability environment had detrimental effects on any transformational effort: the inability for DHBs to allocate resources based on intelligent commissioning; and the inability for DHBs to genuinely engage with communities, in particular Māori, to plan and deliver health services.

DHBs in deficit are unable to invest in capacity and capability for improvement or do so with the risk of being reprimanded by the $\mathrm{MoH}$, the media and political leaders and may face financial penalty from the $\mathrm{MoH}$, or commissioners appointed by the Minister of Health. The latter have wide ranging powers to influence DHB governance, including the ability to replace elected and appointed board members, approve financial and health service delivery plans and stop LST initiatives. The traditional financial monitoring frameworks also affect DHBs' ability to use evidence from commissioning processes to redirect funding as they have to deliver to contract outputs.

The commonly expressed view among the senior operational and clinical leaders at workshops and interview participants working in the health system was that system accountability needed a refresh to shift away from a national template driven approach to one that considers the local conditions of DHBs and provides the flexibility for DHBs to work within defined margins rather than meeting national targets.

... it's an indictment on our health care system now that for decades we've not been able to get beyond deficit and surplus thinking as the overarching framework within which the health care system functions. (IP02) 
Participants said that having margins for DHBs to operate within rather than targets change the conversation around accountability. If DHBs are operating within the defined margins, more flexibility is given to those DHBs and the conversation is around continuous improvement on the quality of care delivered, building learning systems and comparisons among peers. If DHBs are operating below the defined margins, then the conversation is one of performance management and taking swift action to bring performance of those DHBs back within the margins.

A refresh in system accountability would allow intelligent commissioning at the district level and the use of 'plan-do-study-act' cycles to try new initiatives and assess if the innovation is delivering expected results and stop if it is not delivering. The continuous learning approach contributes to the maturity of the system in which a level of failure is allowed to determine future investment and a focus on improving health outcomes and not just financials and targets.

Participants said that transactional relationships hindered genuine engagement with local communities, Māori and Iwi. This is because the priority is on achieving measures and targets that are important to the government or those publicly reported to avoid sanctions and penalties. This focus perpetuates the system being provider centric. Participant IP05 thought that there was both a lack of priority, and the lack of knowledge and understanding about how to engage with local communities, Māori and Iwi. The milestones and key performance indicators in contracts create a perverse incentive of achieving these within the required timeframes, which can sometimes lead to tokenistic consultation with communities, Māori and Iwi. The accountability environment does not encourage or inspire health providers to genuinely engage and reflect the voice of their communities in the governance and delivery of health services. This leads to a lack of focus on designing initiatives to achieve system transformation.

The key mechanisms triggered by the system accountability environment that influences successful implementation of LST initiatives and the outcomes that follow are summarised in Figure 11.11. 


\subsubsection{Training of health care professionals}

The national training programme for health care professionals was identified as a key context that influenced successful implementation of LST initiatives. Two interview participants (IP02 and IP03) discussed the dual role of health care professionals in the system: one to be a qualified and competent health professional; and the other to be a change agent within the health system. Participants said that current training programmes teach health care professionals about their clinical speciality, such as how to diagnose and treat patients. It does not teach health care professionals their other job, which is to be a change agent in the system. This involves working collaboratively with other health care professionals, managers, and patients and communities to improve the quality and experience of care, which requires change management and leadership skills. Participants thought that if those responsible for the national training curriculum understood and acknowledged the dual role of health care professionals in the system, this will lead to changes in the curriculum and health care professionals will be better prepared for their role in the system as change agents.

Change fatigue and lack of resourcing and support causes clinical leaders to disengage. Two workshop participants talked about their experience of clinical leaders being burnt out and "turn in the opposite direction" with the lack of nurturing and mentoring. Participants emphasised the role of professional organisations to create broader opportunities for clinical leaders to "learn the dark art of politics".

\section{Mechanisms}

Participants said it is important that the health care professionals are aware of complexities of the health system from the beginning of their career and understand that providing high-quality health care involves co-ordinated care from across the system and not just the patient in front of them or their speciality (IP02 and IP03). It will help them comprehend the system dynamics 
and constraints that contribute to funding and professional practice decisions and how to navigate the system to improve the quality of care (IP03). This understanding provides a mechanism to implement clinical leadership in practice (IP02). Workshop participants said this understanding also strengthens the relationship between the clinical leaders and the managers and enables them to work together to achieve the shared vision.

The key mechanism triggered by the training of health care professionals that influences successful implementation of LST initiatives and the outcome that follows are summarised in Figure 11.12

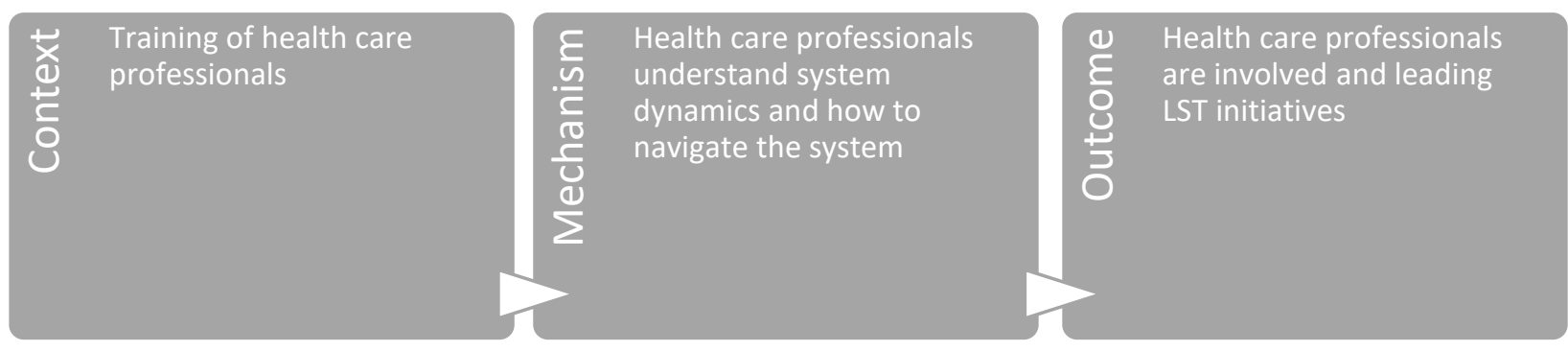

Figure 11.12: CMO theory - Training of health care professionals 


\subsection{Chapter conclusion}

This chapter explained how local and national contexts influence a district's ability to successfully implement LST initiatives in the New Zealand health system. While some of my initial propositions on contexts and mechanisms were confirmed by research participants, evidence from knowledge of those working in the system and leading LST initiatives provided rich descriptions of mechanisms that are triggered by some of the local and national contexts.

There were five local contexts identified in this research: history of working together, distributed leadership from DHBs, maturity of Alliances, capacity and capability, and continuous improvement culture.

Three of the local contexts were inter-related and had a ripple effect on building and maintaining the key mechanism of trust: a history of working together; strong relationships and distributed leadership from DHBs; and the maturity of Alliances. The presence of trust among senior system leaders $(M)$ triggered by a positive history of working together $(C)$ led to strong relationships and DHB senior leaders practising distributed leadership $(0)$. The strong relationships and continued practice of distributed leadership from DHB senior leaders (C) nurtured and sustained trust between senior system leaders (M), and led to these leaders agreeing a shared vision and goals for their local system and a commitment to work towards these through an alliancing way of working (O). The agreement and commitment among senior leaders were enabling contextual factors that increased maturity of Alliances (C). Mature Alliances were able to navigate through disagreements, emerging issues and changes in their membership (M), which led to them being cohesive and resilient and providing a critical platform to successfully implement LST initiatives (O). The key mechanism of trust, built and nurtured over time, is a key mechanism for an alliancing way of working. Figure 11.13, adapted from Jagosh, et al. [179], illustrates this ripple effect. 


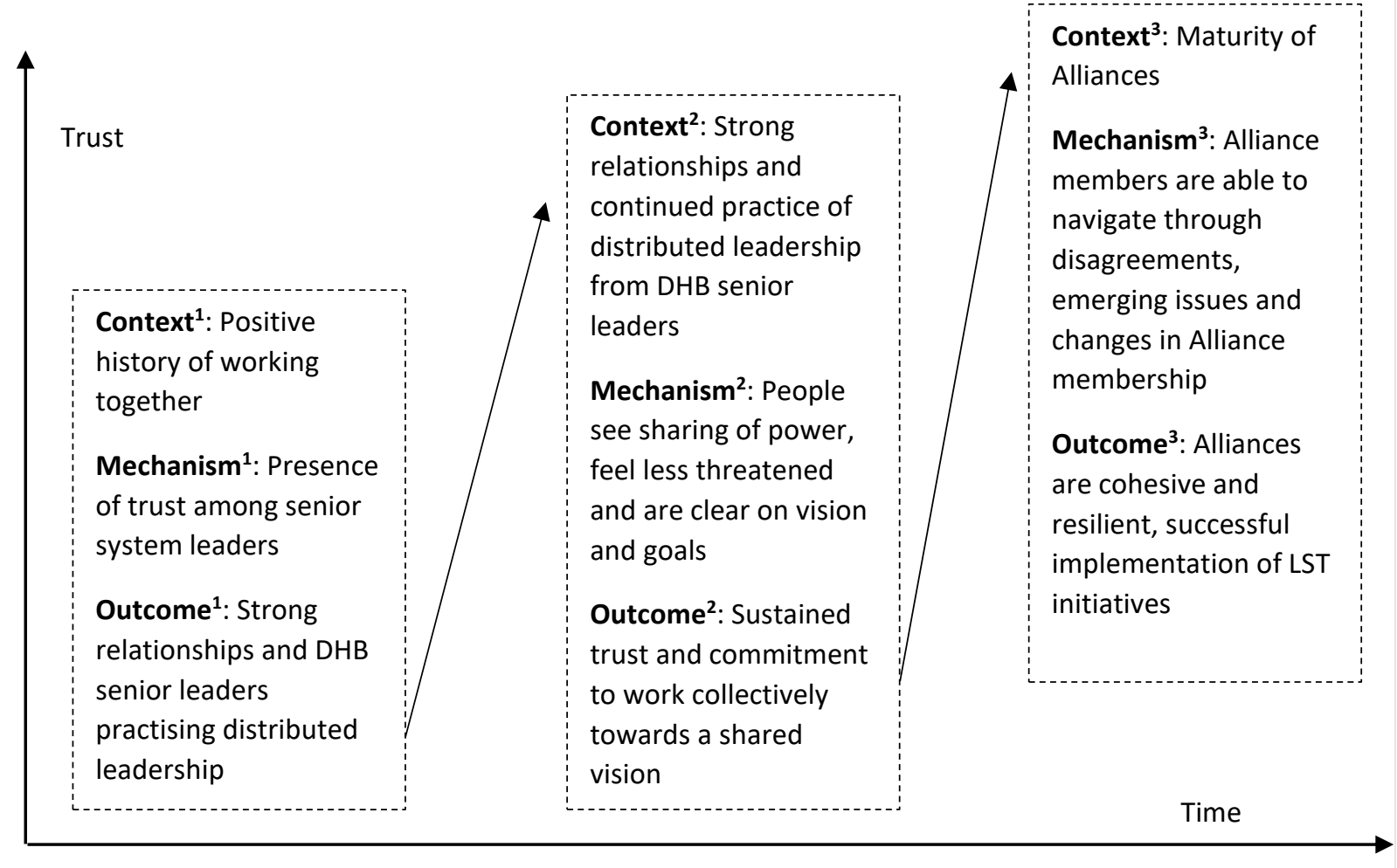

Figure 11.13: CMO interplay showing the ripple effect of the trust mechanism over time, adapted from Jagosh, et al. [179]

The national contexts included the 'new power' approach to the design and implementation of LST initiatives, the political environment, media bias, health system leadership, the funding environment, the system accountability environment, and the training of health care professionals.

Table 11.1 shows the summary of the local and national contexts, the factors that enable and constrain these, the mechanisms triggered and the outcomes that follow, using the realist analysis method adapted from Willis et al. [198]. These CMO theories form part of the programme architecture for successful implementation of LST initiatives and support the notion of a learning system by consciously and deliberately increasing the understanding of health system leaders and Alliances of this architecture to successfully implement LST initiatives in the New Zealand health system. 


\begin{tabular}{|c|c|c|c|c|}
\hline Context & Realist mechanism & Constraining factors & Enabling factors & Outcome \\
\hline $\begin{array}{l}\text { History of working } \\
\text { together and quality of } \\
\text { relationships }\end{array}$ & $\begin{array}{l}\text { Successful with previous change } \\
\text { efforts } \\
\text { Presence of trust among senior } \\
\text { leaders of health system partners } \\
\text { 'Bottom-up' approach to change }\end{array}$ & $\begin{array}{l}\text { Negative history of working together } \\
\text { 'Master-servant' dynamic between } \\
\text { senior leaders in DHBs and PHOs } \\
\text { Power and control by senior DHB and } \\
\text { PHO leaders } \\
\text { Focus on hospital } \\
\text { Top-down approaches to LST initiatives } \\
\text { Frontline health care professionals are } \\
\text { not involved in design of solutions or } \\
\text { their solutions are not implemented by } \\
\text { operational leaders }\end{array}$ & $\begin{array}{l}\text { Positive history of working together } \\
\text { Senior leaders value relationship and } \\
\text { collective action through 'bottom-up' } \\
\text { approaches to LST initiatives } \\
\text { DHB shares power with other system } \\
\text { leaders } \\
\text { DHBs recognise the system outside } \\
\text { the hospital } \\
\text { Frontline health care professionals } \\
\text { are given tools, are involved in } \\
\text { design of solutions and see their } \\
\text { solutions being implemented }\end{array}$ & $\begin{array}{l}\text { Enhanced trust } \\
\text { Enhanced quality of } \\
\text { relationships } \\
\text { DHB senior leaders practise } \\
\text { distributed leadership } \\
\text { Clinical and operational leaders } \\
\text { are more willing to come } \\
\text { together, share resources, data } \\
\text { and ideas and attempt on } \\
\text { change efforts } \\
\text { Clinicians feel empowered to } \\
\text { improve the system they work } \\
\text { in }\end{array}$ \\
\hline $\begin{array}{l}\text { Distributed leadership } \\
\text { from DHB leaders }\end{array}$ & $\begin{array}{l}\text { People see sharing of power and } \\
\text { feel less threatened by the size of } \\
\text { organisation or budget } \\
\text { Fosters a collective approach to } \\
\text { implementation of LST initiatives } \\
\text { Staff are clear on vision and goals } \\
\text { and use these to prioritise their } \\
\text { work and feel confident to do } \\
\text { things differently }\end{array}$ & $\begin{array}{l}\text { Low-trust relationships between DHBs } \\
\text { and PHOs } \\
\text { Command and control leadership style } \\
\text { of senior leaders in DHBs and PHOs } \\
\text { Power and control by senior DHB and } \\
\text { PHO leaders } \\
\text { Poor history of working together } \\
\text { Focus on hospital } \\
\text { Change fatigue }\end{array}$ & $\begin{array}{l}\text { High-trust relationships between } \\
\text { DHBs and PHOs } \\
\text { Collaborative, collective or } \\
\text { distributed leadership styles of } \\
\text { senior leaders in DHBs and PHOs } \\
\text { Positive history of working together } \\
\text { Strong and courageous leadership } \\
\text { DHBs recognise the system outside } \\
\text { the hospital }\end{array}$ & $\begin{array}{l}\text { Trust between senior system } \\
\text { leaders is nurtured and } \\
\text { sustained } \\
\text { Senior leaders in the district } \\
\text { agree on an agreed integrated } \\
\text { work programme for their } \\
\text { district that includes a shared } \\
\text { vision and goals; and all actors } \\
\text { commit to working towards the } \\
\text { agreed work programme }\end{array}$ \\
\hline Maturity of Alliances & $\begin{array}{l}\text { Alliance members are able to } \\
\text { navigate through disagreements, } \\
\text { deal with emerging issues, } \\
\text { overcome changes in senior } \\
\text { leadership roles and Alliance } \\
\text { membership, and stay focused } \\
\text { towards the shared vision }\end{array}$ & $\begin{array}{l}\text { Lack of an Alliance } \\
\text { Lack of a whole-of-system approach } \\
\text { Lack of a shared vision and goals } \\
\text { Lack of resources to support the } \\
\text { Alliance } \\
\text { Lack of sharing data and intelligence }\end{array}$ & $\begin{array}{l}\text { High-trust relationships } \\
\text { Shared vision and goals } \\
\text { Commitment among Alliance } \\
\text { partners to work towards shared } \\
\text { vision and goals }\end{array}$ & $\begin{array}{l}\text { Alliance is cohesive and } \\
\text { resilient and is able to } \\
\text { successfully implement LST } \\
\text { initiatives }\end{array}$ \\
\hline
\end{tabular}




\begin{tabular}{|c|c|c|c|c|}
\hline \multirow[t]{2}{*}{ Context } & Realist mechanism & Constraining factors & Enabling factors & Outcome \\
\hline & & $\begin{array}{l}\text { Agreed Alliance charter in place but } \\
\text { behaviours of members do not reflect } \\
\text { the way of working described in the } \\
\text { charter }\end{array}$ & $\begin{array}{l}\text { Members of the Alliance 'live' the } \\
\text { agreed charter } \\
\text { Alliance has independent chair. } \\
\text { Alliance has mandate and embedded } \\
\text { within DHB governance structure } \\
\text { Alliance is supported by lower level } \\
\text { structures such as working groups }\end{array}$ & \\
\hline Capacity and capability & $\begin{array}{l}\text { System leaders recognise the } \\
\text { importance of capacity and } \\
\text { capability for implementation of } \\
\text { LST initiatives } \\
\text { Use of data and evidence in } \\
\text { change efforts } \\
\text { Health care professionals see } \\
\text { value in the use of integrated } \\
\text { health information }\end{array}$ & $\begin{array}{l}\text { Lack of awareness by senior leaders on } \\
\text { the capacity and capability of their } \\
\text { organisation } \\
\text { Power and control by senior DHB and } \\
\text { PHO leaders } \\
\text { Frequent changes to senior leadership } \\
\text { roles } \\
\text { Poor relationships } \\
\text { Lack of dedicated analytic resources, } \\
\text { skilled project or programme } \\
\text { managers, budget and evaluative } \\
\text { processes } \\
\text { Lack of or out of date appropriate } \\
\text { information technology tools } \\
\text { Clinicians not reimbursed for their loss } \\
\text { of income or costs to attend meetings } \\
\text { Change management added to existing } \\
\text { work demands of those in the system }\end{array}$ & $\begin{array}{l}\text { Continuity of system leadership roles } \\
\text { Senior leaders value relationships } \\
\text { High-trust relationships } \\
\text { Willingness to share power, } \\
\text { successes and failures Integrated } \\
\text { health information } \\
\text { Analytic capability } \\
\text { Dedicated personnel with right skills } \\
\text { recruited or re-directed to co- } \\
\text { ordinate and or assist with change } \\
\text { efforts } \\
\text { Time and incentives available for } \\
\text { clinical leaders to engage in Alliance } \\
\text { leadership team } \\
\text { Meetings held at times suitable for } \\
\text { clinicians and frontline staff to } \\
\text { attend } \\
\text { Clinicians provided with locum to } \\
\text { backfill } \\
\text { Clinicians reimbursed for their loss of } \\
\text { income and costs to attend meetings }\end{array}$ & $\begin{array}{l}\text { System leaders invest in } \\
\text { organisational capacity and } \\
\text { capability } \\
\text { Willingness of system leaders } \\
\text { to partner with other } \\
\text { organisations to boost capacity } \\
\text { and capability } \\
\text { Stronger clinical engagement in } \\
\text { identification of meaningful } \\
\text { quality improvement activities } \\
\text { Health care professionals more } \\
\text { likely to share patient level } \\
\text { data across the system }\end{array}$ \\
\hline
\end{tabular}




\begin{tabular}{|c|c|c|c|c|}
\hline Context & Realist mechanism & Constraining factors & Enabling factors & Outcome \\
\hline $\begin{array}{l}\text { Continuous } \\
\text { improvement culture }\end{array}$ & $\begin{array}{l}\text { Active support from senior } \\
\text { system leaders for a continuous } \\
\text { improvement approach } \\
\text { Staff feel encouraged and } \\
\text { supported to challenge status } \\
\text { quo and try new initiatives }\end{array}$ & $\begin{array}{l}\text { Sole focus on accountability reporting } \\
\text { Lack of organisational values or values } \\
\text { do not support continuous } \\
\text { improvement } \\
\text { Lack of awareness, availability and use } \\
\text { of improvement science tools and } \\
\text { methodology } \\
\text { Lack of buy-in from senior system } \\
\text { leaders } \\
\text { Culture of blame }\end{array}$ & $\begin{array}{l}\text { Balance between accountability and } \\
\text { continuous improvement } \\
\text { Organisational values support } \\
\text { continuous improvement } \\
\text { Use of constant feedback loops and } \\
\text { plan-do-study-act processes } \\
\text { Use of evaluation processes } \\
\text { A permissive culture to try new } \\
\text { initiatives (i.e., 'Safe to fail') }\end{array}$ & $\begin{array}{l}\text { Organisations embrace critique } \\
\text { and look to continuously } \\
\text { improve their performance }\end{array}$ \\
\hline $\begin{array}{l}\text { 'New power' approach } \\
\text { to design and } \\
\text { implementation of LST } \\
\text { initiatives }\end{array}$ & $\begin{array}{l}\text { Confidence in system actors to be } \\
\text { innovative and do things } \\
\text { differently }\end{array}$ & $\begin{array}{l}\text { Lack of dedicated resources and time } \\
\text { Lack of political mandate and credibility } \\
\text { Lack of financial incentives } \\
\text { Disconnect between national policies } \\
\text { and planning processes }\end{array}$ & $\begin{array}{l}\text { Dedicated resources and time } \\
\text { National improvement or seed } \\
\text { funding available } \\
\text { Collaborative development and } \\
\text { implementation of the LST initiatives } \\
\text { Financial incentives }\end{array}$ & $\begin{array}{l}\text { Sustained engagement with } \\
\text { implementation of LST } \\
\text { initiatives }\end{array}$ \\
\hline Election cycle & $\begin{array}{l}\text { Short-term focus on system } \\
\text { transformation }\end{array}$ & $\begin{array}{l}\text { Three-year election cycle } \\
\text { Rapid change in national policies and } \\
\text { budget packages } \\
\text { Constant reforms with change of } \\
\text { governments } \\
\text { Use of pilot studies } \\
\text { Change in key leadership roles }\end{array}$ & $\begin{array}{l}\text { Presence of high-level think tank } \\
\text { Shared vision for the system } \\
\text { Long-term planning that is enduring } \\
\text { to change in government }\end{array}$ & Disruption to LST initiatives \\
\hline
\end{tabular}




\begin{tabular}{|c|c|c|c|c|}
\hline Context & Realist mechanism & Constraining factors & Enabling factors & Outcome \\
\hline $\begin{array}{l}\text { National policy } \\
\text { environment }\end{array}$ & $\begin{array}{l}\text { Change fatigue that creates } \\
\text { resistance to change or inertia in } \\
\text { the system }\end{array}$ & $\begin{array}{l}\text { Lack of shared vision or clear strong } \\
\text { foundation for the system } \\
\text { The MoH does not share with the } \\
\text { sector the policy advice given to } \\
\text { Ministers and their response to it (lack } \\
\text { of transparency) } \\
\text { Change in key leadership roles such as } \\
\text { the Director General of Health }\end{array}$ & $\begin{array}{l}\text { Presence of high-level think tank } \\
\text { Shared vision for the system through } \\
\text { collaborative policy design } \\
\text { Long-term planning that is enduring } \\
\text { to change in government } \\
\text { Transparency in policy advice } \\
\text { provided to the Ministers }\end{array}$ & $\begin{array}{l}\text { Inertia in the system blocks or } \\
\text { resists LST initiatives }\end{array}$ \\
\hline Government ideology & $\begin{array}{l}\text { Collaborative or competitive } \\
\text { behaviour that follows the } \\
\text { government ideology }\end{array}$ & $\begin{array}{l}\text { Competitive funding environment that } \\
\text { erodes trust and collective action on } \\
\text { shared vision } \\
\text { Different philosophical underpinnings } \\
\text { of other government agencies which } \\
\text { influence wider social determinants of } \\
\text { health }\end{array}$ & $\begin{array}{l}\text { Collaborative ideology } \\
\text { Policies and agendas of other } \\
\text { government agencies are aligned } \\
\text { with the health system } \\
\text { Shared vision for the health system } \\
\text { Funding environment that builds } \\
\text { trust and generates collective action } \\
\text { towards shared vision }\end{array}$ & $\begin{array}{l}\text { Government ideology } \\
\text { influences LST initiatives (can } \\
\text { be positive or negative } \\
\text { influence) }\end{array}$ \\
\hline Media bias & $\begin{array}{l}\text { Fosters a blame culture that } \\
\text { suffocates the notion of a } \\
\text { learning system }\end{array}$ & $\begin{array}{l}\text { Public reprimand of senior leaders for } \\
\text { not meeting accountability goals } \\
\text { Lack of shared vision or clear strong } \\
\text { foundation for the system }\end{array}$ & $\begin{array}{l}\text { Presence of high-level think tank } \\
\text { Shared vision for the system } \\
\text { Long-term planning that is enduring } \\
\text { to change in government }\end{array}$ & $\begin{array}{l}\text { Senior leaders do not support } \\
\text { or engage in LST initiatives }\end{array}$ \\
\hline $\begin{array}{l}\text { Health system } \\
\text { leadership }\end{array}$ & $\begin{array}{l}\text { Fostering collective system } \\
\text { leadership } \\
\text { Supporting and celebrating } \\
\text { innovations }\end{array}$ & $\begin{array}{l}\text { Lack of shared vision or clear strong } \\
\text { foundation for the system } \\
\text { Unclear expectations of system leaders } \\
\text { The MoH's performance monitoring } \\
\text { frameworks focus on DHB bottom line } \\
\text { Service improvements are often seen } \\
\text { as "woolly and soft" and a nice to have } \\
\text { "when the DHB bottom line is sorted" }\end{array}$ & $\begin{array}{l}\text { Presence of high-level think tank } \\
\text { Clear expectations of system leaders } \\
\text { Long-term planning that is enduring } \\
\text { to change in government } \\
\text { The MoH embracing the } \\
\text { improvement function } \\
\text { Peer mentoring and support through } \\
\text { learning communities } \\
\text { Culture of continuous improvement }\end{array}$ & $\begin{array}{l}\text { Builds system leadership } \\
\text { capacity and capability and } \\
\text { facilitates collaborative } \\
\text { learning } \\
\text { Supports the notion of a } \\
\text { learning system }\end{array}$ \\
\hline
\end{tabular}




\begin{tabular}{|c|c|c|c|c|}
\hline Context & Realist mechanism & Constraining factors & Enabling factors & Outcome \\
\hline Funding environment & $\begin{array}{l}\text { Provides a motivator or the } \\
\text { burning platform for LST } \\
\text { initiatives }\end{array}$ & $\begin{array}{l}\text { No reason to change } \\
\text { Everyone protects their own budget } \\
\text { Micromanagement of budgets } \\
\text { Funding seen as the sole catalyst for } \\
\text { change } \\
\text { Continued focus on DHB bottom line } \\
\text { Lack of transparency on how incentive } \\
\text { funding is used } \\
\text { Inconsistent use of incentive payments }\end{array}$ & $\begin{array}{l}\text { System leaders understanding the } \\
\text { financial context } \\
\text { Natural disasters } \\
\text { National fund together with an } \\
\text { improvement team to LST initiatives } \\
\text { Systemic and transparent use of } \\
\text { incentive funding to support LST } \\
\text { initiatives }\end{array}$ & $\begin{array}{l}\text { Funding is seen as an enabler } \\
\text { for innovative thinking }\end{array}$ \\
\hline $\begin{array}{l}\text { System accountability } \\
\text { environment }\end{array}$ & $\begin{array}{l}\text { System leaders place more effort } \\
\text { on achieving accountability } \\
\text { measures to avoid sanctions and } \\
\text { penalties } \\
\text { System actors feel disempowered } \\
\text { to involve their local } \\
\text { communities, Māori and Iwi }\end{array}$ & $\begin{array}{l}\text { National template driven approach to } \\
\text { system accountability } \\
\text { Output based performance measures } \\
\text { Focus on DHB financial position } \\
\text { Penalties and sanctions for not meeting } \\
\text { performance or financial targets } \\
\text { Lack of priority on engaging with local } \\
\text { communities, Māori and Iwi } \\
\text { Tokenistic consultation with local } \\
\text { communities, Māori and Iwi owing to } \\
\text { time constraints to deliver on } \\
\text { contractual requirements } \\
\text { Lack of skills and knowledge on how to } \\
\text { genuinely engage with local } \\
\text { communities, Māori and Iwi }\end{array}$ & $\begin{array}{l}\text { System accountability frameworks } \\
\text { allow flexibility and consider local } \\
\text { contexts } \\
\text { Safety margin rather than national } \\
\text { target approach } \\
\text { Focus on whole health system } \\
\text { performance and funding } \\
\text { Staff capability and capacity for } \\
\text { genuine engagement } \\
\text { Local communities, Māori and Iwi } \\
\text { are involved in governance and } \\
\text { delivery of health services } \\
\text { Awareness and acknowledgement of } \\
\text { Te Tiriti obligations }\end{array}$ & $\begin{array}{l}\text { System performance } \\
\text { monitoring and reporting is } \\
\text { focused on accountability } \\
\text { measures and not on } \\
\text { continuous improvement } \\
\text { Local communities, Māori and } \\
\text { Iwi are unable to be involved in } \\
\text { LST initiatives }\end{array}$ \\
\hline $\begin{array}{l}\text { Training of health care } \\
\text { professionals }\end{array}$ & $\begin{array}{l}\text { Health care professionals } \\
\text { understand system dynamics and } \\
\text { how to navigate the system }\end{array}$ & $\begin{array}{l}\text { Health care professionals are not } \\
\text { taught about their role as change } \\
\text { agents in the health system } \\
\text { Lack of change management and } \\
\text { leadership skills }\end{array}$ & $\begin{array}{l}\text { Health care training curriculum } \\
\text { teaches health care professionals } \\
\text { about their role as change agents in } \\
\text { the health system }\end{array}$ & $\begin{array}{l}\text { Health care professionals are } \\
\text { involved and leading LST } \\
\text { initiatives }\end{array}$ \\
\hline
\end{tabular}




\begin{tabular}{|c|c|c|c|c|}
\hline Context & Realist mechanism & Constraining factors & Enabling factors & Outcome \\
\hline & & $\begin{array}{l}\text { Health care professionals focus on their } \\
\text { patients and their speciality } \\
\text { Lack of clinical leadership opportunities }\end{array}$ & $\begin{array}{l}\text { Change management and leadership } \\
\text { skills } \\
\text { Clinical leadership opportunities } \\
\text { Peer support and mentoring } \\
\text { programmes }\end{array}$ & \\
\hline
\end{tabular}

Table 11.1: Consolidated CMO theories for successful implementation of LST initiatives in the New Zealand health system 


\subsection{Summary of Part C}

Part C presented the research findings. Using realist research design shown in

Figure 7.1 (Chapter 7), this part of the thesis answered the three research questions and analysed and described the programme architecture that underpins efforts to successfully implement LST initiatives.

Chapter 9 identified 10 key elements that are needed to increase the chances of successful implementation of LST initiatives in the New Zealand health system. These are: an alliancing way of working; a commitment to Te Tiriti o Waitangi; clinical leadership and involvement; integrated health information; analytic capability; intelligent commissioning; a continuous improvement; an understanding of equity; dedicated resources and time; and involved people, whānau and community.

Chapter 10 presented the self-assessment maturity matrix for the 10 elements, constructed with senior clinical and operational leaders working in the New Zealand health system. The maturity matrix was tested with three Alliance leadership teams. The maturity matrix is designed to consciously and deliberately increase health system leaders' and Alliances' understanding of what these key elements look like for the New Zealand health system. My findings suggest that the maturity matrix enables Alliances to assess where they are on the maturity scale for each element; and identify areas for improvement. The research literature suggests that a regular selfassessment process will create feedback loops and support the notion of a learning system for the New Zealand health system.

Chapter 11 drilled down into the elements and reported on how local and national contexts influence a district's ability to successfully implement LST initiatives. The chapter presented CMO theories that reported on mechanisms triggered by particular contexts and the outcomes that follow for successful implementation of LST initiatives. At a local level, the history of working together and quality of relationships, distributed leadership from DHBs, and the maturity of Alliances emerged as key local contexts that influence successful implementation of LST initiatives. These contexts trigger the key mechanism of trust that must be built and maintained over time to successfully implement LST initiatives.

At a national level, the 'new power' approach to design and implementation of LST initiatives, system leadership, and the system accountability environment emerged as key contexts that influenced districts' ability to successfully implement LST initiatives. System leaders create the 
right conditions for collective responsibility of LST initiatives using 'new power' approach. The 'new power' approach generates power from relationships, connections and the ability to influence through informal networks and communities to achieve a shared vision. It relies on emotional connection that uses people's intrinsic motivation to call for action. Most importantly, it engages whānau, local communities, Māori, Iwi, and frontline health care professionals in transformational change.

The next part of the thesis discusses key findings of the research and concludes the research with recommendations emerging from the research. 
PART D: DISCUSSION AND CONCLUSIONS 
Part D of the thesis contains four chapters: one substantial and three short chapters.

\section{Chapter 12: Key research findings}

This chapter summarises key findings of the research. It discusses how an alliancing way of working is the key element that needs to be present to increase the chances of success with implementation of LST initiatives. Further, the chapter goes on to discuss the key contextual factors that influence successful implementation of LST initiatives. By successful implementation of LST initiatives I am referring to the intermediate outcomes discussed in section 1.5 (Chapter 1). Chapter 12 pays attention to local contexts that include a history of working together, distributed leadership from DHBs and the maturity of Alliances; and national contexts that include the 'new power' approach to design and implementation of LST initiatives, system leadership, and the system accountability environment.

\section{Chapter 13: Recommendations}

This chapter outlines five recommendations emerging from the research. These relate to a collaborative way of working, use of the maturity matrix to increase the health system leaders' understanding of the programme architecture that underpins efforts to successfully implement LST initiatives, development of a national improvement team underpinned by 'Human Learning Systems' concept; a national improvement budget; and a flexible accountability framework that enables communities to be involved in the design and implementation of LST initiatives.

\section{Chapter 14: Strengths and limitations}

This chapter identified the strengths and limitations of the research. My knowledge and insights from leading implementation of the SLM programme was a key strength, which allowed me to recruit participants that were information rich. However, the purposeful sampling technique, the small number of participants for the workshops and the maturity matrix being tested with only three Alliance leadership teams limited the range of views in the research.

\section{Chapter 15: Concluding chapter}

This is the final chapter that concludes the research. Along with concluding remarks, the chapter discusses the future of the SLM programme, contribution of this research and identifies opportunities for future research. 


\subsection{KEY RESEARCH FINDINGS}

This research analysed and described the programme architecture that underpins efforts to successfully implement LST initiatives in the New Zealand health system using insights gained from the implementation of the SLM programme. The SLM programme is an example of an LST initiative.

The programme architecture includes key elements that need to be present to increase the chances of successful implementation of LST initiatives. A self-assessment maturity matrix was constructed with knowledge of those working in the system. This matrix described the key elements along a maturity scale to increase the understanding of health system leaders and Alliances and in doing so, support the notion of a learning system. Further, the research drilled further and reported on how these elements work in different contexts to influence the successful implementation of LST initiatives. This programme architecture bridges the gap between theory of LST initiatives and the reality of successfully implementing these initiatives in the New Zealand health system.

LST initiatives embrace a systems mind-set and mobilise many agents present in a system to work towards a shared vision. The goal of LST initiatives in health systems is to achieve the desired system transformation.

This chapter discusses the key themes that emerged from the research findings: an alliancing way of working that provides a critical platform to successfully implement LST initiatives; and the selfassessment maturity matrix, which acts as a practical tool to consciously and deliberately increase Alliances' understanding of the programme architecture. The chapter then discusses the key local and national contexts that influence successful implementation of LST initiatives. The key local contextual factors identified were a history of working together, distributed leadership from DHBs and the maturity of Alliances. The key national contextual factors identified were system leadership, system accountability environment, the 'new power' approach to design and implementation of LST initiatives.

\subsection{Key elements}

Best, et al. [1] called it "simple rules"; Willis, et al. [198] referred to it as the "guiding principles"; Evans, et al. [3] called it the "organisational capabilities"; Baker and Axler [122] called it "key attributes"; Perla, et al. [30] referred to it as "primary and secondary drivers"; and McGrath, et al. [190] called it "key elements". They are all referring to a set of elements that need to be 
present in complex adaptive systems and work in harmony to increase the chances of success with implementation of LST initiatives.

The research identified 10 key elements that need to be present in the New Zealand health system to increase the chances of success with implementation of LST initiatives: an alliancing way of working; a commitment to Te Tiriti o Waitangi; clinical leadership and involvement; involved people, whānau and communities; integrated health information; analytic capability; dedicated resources and time; intelligent commissioning; understanding of equity; and continuous improvement.

Five of the elements identified in this research were identified in other studies of LST initiatives. Table 12.1 below shows key elements from this research mapped to similar elements from literature.

Other elements identified in the literature included: leadership approach; accountability and performance measurement; attending to history; readiness for change; organisational culture; delivery of patient-centred care; vision and aim; commitment and responsibility; and building and maintaining a proficient workforce. In this research, most of these elements were identified as contextual factors or mechanisms that enable successful implementation of LST initiatives. Appendix 2 shows the full list of elements identified in the 10 studies that involved implementation of a large-scale change. 


\begin{tabular}{|c|c|}
\hline $\begin{array}{l}\text { Key element identified in this } \\
\text { research }\end{array}$ & Key element identified in literature \\
\hline An alliancing way of working & $\begin{array}{l}\text { Enhancing system governance [122] } \\
\text { Common goals, beliefs and values among stakeholders [199] } \\
\text { Partnering [3] } \\
\text { Relationships and networks [141] } \\
\text { Alliance; Teams and professions working together to achieve } \\
\text { common goals [200] } \\
\text { Collaborative decision-making and relationships between health } \\
\text { professionals [37] } \\
\text { Create collaborative relationships [198] }\end{array}$ \\
\hline $\begin{array}{l}\text { Clinical leadership and } \\
\text { involvement }\end{array}$ & $\begin{array}{l}\text { Engage physicians [1] } \\
\text { Enhancing professional cultures and engaging clinicians [122] } \\
\text { Clinical engagement and leadership [3] } \\
\text { Clinical leadership [190] } \\
\text { Clinical leadership and engagement [200] }\end{array}$ \\
\hline $\begin{array}{l}\text { Involved people, whānau and } \\
\text { communities }\end{array}$ & $\begin{array}{l}\text { Include patients and families [1] } \\
\text { Engaging patients, caregivers and the public [122] }\end{array}$ \\
\hline Integrated health information & $\begin{array}{l}\text { Enabling comprehensive information infrastructures [122] } \\
\text { Information technology [3] } \\
\text { Knowledge and data [141] } \\
\text { Access to data [190] } \\
\text { Data infrastructure [30] } \\
\text { IT infrastructures [200] } \\
\text { Investing in integrated electronic health records [37] }\end{array}$ \\
\hline Analytic capability & $\begin{array}{l}\text { Enabling comprehensive information infrastructures [122] } \\
\text { Knowledge and data [141] }\end{array}$ \\
\hline Dedicated resources and time & $\begin{array}{l}\text { Investing in capacity to support improvement [122] } \\
\text { Resources }[3,30,141,199]\end{array}$ \\
\hline Intelligent commissioning & Stratifying population by risk [37] \\
\hline Continuous improvement & $\begin{array}{l}\text { Establish feedback loops [1] } \\
\text { Focusing on quality and system improvement [122] } \\
\text { Commitment to learning and improving quality [3] } \\
\text { Learning networks; Measurement and feedback systems [30] } \\
\text { A process for maintaining improvement [190] } \\
\text { Using data for continuous quality improvement [200] } \\
\text { Continuously assess and learn from change [198] }\end{array}$ \\
\hline Understanding of equity & Attending to access and equity issues [122] \\
\hline
\end{tabular}

Table 12.1: Key elements from this research mapped to similar elements from literature

While Table 12.1 shows elements that were commonly identified in the literature, it also highlights areas where this research adds value to health system transformation literature. Although studies identified patient-centred care as an important element for successfully implementing LST initiatives, only two studies specified this to include or engage patients and their families in the change efforts. The rest focussed on delivering co-ordinated or patientcentred care. Similarly, while integrated health information was commonly identified, analytic capability was not. As mentioned earlier in the thesis, having integrated health information is not 
enough; the ability to produce knowledge and insights from information is critical to implementing LST initiatives. Without the ability to analyse, interpret and use data, even the best quality data could be useless [19]. In New Zealand, there is a shortage of people in public sector with analytic skills required to meet the future demand for these services [19].

Intelligent commissioning was not mentioned in any of the studies; this may be owing to poor or variable understanding of what this element involved, as was the case with participants in this research. According to NHS Scotland [192], it is the numerous definitions of the term 'commissioning' that continue to be contested over the last two decades rather than the activities involved in commissioning. Intelligent commissioning is an important element to ensure that resources are targeted appropriately to needs and investment is linked to agreed outcomes $[189,192]$. It is different from contracting. The four key strands of commissioning are: using data to understand long and short-term needs; planning innovative solutions to meet the needs identified; delivering and monitoring services using contracts and providers; and using performance data to adjust, add or drop services [19]. Underpinning commissioning is continuous improvement that involves evaluation of programmes and initiatives to identify successes and failures and sharing these openly so others can learn from [19]. Intelligent commissioning could help shift government accountability from a focus on spending to a wider focus on the quality of spending and achievement of health outcomes [19].

The remaining two elements - an understanding of equity, and a commitment to Te Tiriti o Waitangi - were not specifically found in the literature as key elements that support successful implementation of LST initiatives. In saying that, delivering high quality health care by definition often includes providing equitable health care and therefore, equity is inherent to the continuous quality improvement process and is critical to delivering an integrated person-centred equitable health care [17]. Te Tiriti o Waitangi is a foundational document for New Zealand and therefore understandably not mentioned in international studies of health care transformations.

For New Zealand, where Māori, as the indigenous people, persistently experience poor health outcomes with a higher burden of disease and a lower life expectancy when compared to nonMāori. The commitment to Te Tiriti o Waitangi is a Crown responsibility and is fundamental to any LST initiative in the New Zealand health system $[77,78]$. There was strong support from research participants for this element to be evident rather than presumed or inconspicuously part of another key element. The separation of equity and Te Tiriti elements recognised Te Tiriti principle of equity in modern day health system and emphasised biculturalism (Māori and 
Pākehā) in a multi-cultural setting. Participants felt that equity within the context of Te Tiriti is sometimes poorly understood by staff and leaders in the health system. In an increasingly multicultural country, staff and leaders may perceive equity as providing equitable health care for all high priority populations that include Māori, and sometimes overlook the responsibility towards Māori that comes from Te Tiriti.

\subsubsection{An alliancing way of working}

Although the 10 key elements were not weighted in my analysis, the alliancing way of working emerged as the key element that provided a critical platform for successful implementation of LST initiatives in the New Zealand health system. The presence of the remaining nine elements contribute to successfully implementing change at an organisational or a service level but do not on their own lead to successful implementation of LST initiatives that involve many organisations present in a complex system.

There are other local, regional and national informal networks present in the New Zealand health system, but these are disease- or organisation-focused, such as DHB consumer councils, regional cancer networks and PHO quality improvement networks. In the current policy environment, Alliances are the informal networks that bring system actors together to work beyond their organisational and professional boundaries to achieve a shared vision or goal.

My findings confirm evidence in literature that the desired health system transformation to deliver equitable health outcomes is more likely to be achieved through inter-organisational collaboration rather than competitive behaviours $[1,3,67,122]$. Inter-organisational collaboration involves an alliancing or a collaborative way of working between all agents in the health system towards a shared vision [67].

The Health and Disability System Review noted that a more collaborative approach was needed to achieve equitable health outcomes and to become a more financially sustainable system [23]. The Interim Report of the Review [96] highlighted the transactional paradigm of the New Zealand health system in which the nature and requirement of planning for DHBs is fragmented and focuses on provision of particular services. The report identified a need for a shift in this paradigm that would enable DHBs to undertake long-term planning starting with a population health needs assessment, knowing how these needs are currently being met, followed by re-prioritising to deliver equitable health and wellbeing outcomes for all New Zealanders. The report reinforced that an increase in funding alone will not improve the quality of service or equity in health outcomes. Instead, the report embraced a systems mind set and emphasised that a cohesive, 
integrated system that works in a collaborative, collective and co-operative way is more likely to deliver on equitable outcomes.

However, the final report [23] recommended that DHBs would no longer be required to form Alliances with PHOs delivering primary care services in their districts. This is on the premise that DHBs would no longer be required to contract the delivery of primary care services exclusively from PHOs. The report proposed that Alliances are replaced with a locality approach for planning and delivering primary and community health services (tier one), integrating tier one services with hospital and specialist services (tier two), and monitoring health outcomes through joint accountability. DHBs would be responsible for locality planning for their geographically defined populations. This recommendation removes the exclusivity that exists between DHBs and PHOs to form Alliances.

My insights and the post implementation review of the SLM programme [121] showed that New Zealand Alliances vary in form and function. Some Alliances exist to meet the MoH contractual requirements or are a policy mechanism for PHOs and general practitioners to access the flexible funding pool, and the contractual requirement enabled an exclusivity in the Alliance membership. However, there were districts that did not encounter the contractual requirement as a barrier and had Alliance leadership teams that included broader health system partners including Iwi and community perspectives. My insights from the SLM programme showed that in districts where relationships between DHB and its system partners (in particular PHOs) were fraught, Alliances were perceived as bureaucratic networks that lacked mandate and resources and were ineffective in delivering any benefits for the local system. It is therefore not surprising for the shift away from Alliances by the Health and Disability System Review panel to a locality approach that is more inclusive of system partners involved in the delivery of health care. However, my insights and the evidence in literature also showed some Alliances were successful with implementation of the SLM programme and delivered significant benefits for some DHBs, for example, the Canterbury DHB's transformation journey [68]. Research participants expressed that PHOs, as meso layer organisations, provided a counterbalance to hospital centric services through the power of alliancing.

The recommendations in the final Health and Disability System Review report provided little detail on how the locality approach would work in practice, besides a change in name for Alliances and removing the exclusivity of membership to DHBs and PHOs. Findings from this research confirmed that for networks, such as Alliances, to be successful they needed to be place- 
based and close to the populations they serve. Further, that contexts such as a history of working together, the leadership style of DHB senior leaders, and the maturity of these networks had a more profound influence on their success rather than a national policy direction. This is a common finding from the policy implementation literature: that ideas for change rarely filter through a complex adaptive system without being adapted and re-worked by those on the frontline [201] and that contextual factors, such as history of working together and leadership style, heavily influence the performance of networks such as Alliances [67].

Alliances or locality networks also need to consist of people who will bring local community perspectives, and system leaders who will think beyond their unit, organisation or profession [67]. These networks also need to be embedded in the health system at an executive management level to influence the commissioning decision-making processes. If the upcoming reform is successful in reducing the number of DHBs, the 'super' DHBs will be further removed from the communities they serve and may have multiple locality networks operating in each district. It is unclear how the locality networks in the newly formed 'super' DHB districts will link with each other and with their DHB. Health New Zealand and DHBs will be responsible for creating the right conditions for these locality networks to function and thrive. They will need guidance on the way of working that will give effect to the proposed shared system values (Box 2.2) and achieve aspirations of their communities. The maturity matrix constructed in this research provides the current Alliances and the future locality networks with a practical quality improvement tool to stimulate conscious and deliberate learning about the programme architecture that underpins efforts to successfully implement LST initiatives.

\subsection{The self-assessment maturity matrix}

The maturity matrix was designed to increase the understanding of health system leaders and Alliances about the key elements and assess their readiness and receptiveness for change. Research findings confirmed that the maturity scale for the key elements and the self-assessment process supports the notion of a learning system that enables Alliances to assess where they are and how to get 'from here to there'. Testing with Alliance leadership teams revealed that the maturity matrix stimulated Alliances to self-reflect on where they were along the maturity scale for each element and identify areas of improvement. Such a reflection process could be used prospectively to gauge readiness for change, in real time when implementing change, and retrospectively to understand failures or partial successes of change efforts [161]. Continuous use of the self-reflection process, along with key actions to improve, should build capacity and 
capability of networks to adopt complex innovations (such as LST initiatives) and sustain these long-term at an organisational and at the system level [155-161].

The field-testing process itself was influenced by some of the local contextual factors that emerged in the research. For example, where Alliance leadership teams had high-trust relationships and a positive history of working together, the focus of testing was on the improvement opportunities for the Alliance leadership team. Conversely, when Alliance leadership teams did not have a positive history of working together and trust was low, the focus was on getting the right score, debating the maturity scale ranges, and emphasis put on organisations rather than the system.

The post implementation review of the SLM programme [121] concluded that maturity of Alliance was a pre-condition to enhance a culture of system integration and quality improvement. Therefore, the use of the maturity matrix by Alliances for learning is likely to be dependent on their maturity. More mature Alliances are likely to use the maturity matrix for their continuous improvement while less mature Alliances are less likely to be motivated to use it. The lack of motivation could be because of a lack of understanding of the programme architecture for successful implementation of LST initiatives or a simple lack of willingness owing to the leadership style of the DHB senior leaders and low-trust relationships or simply a lack of insight as to their weaknesses and the need to improve. These Alliances are likely to need peer support and influence to recognise the value of such a tool.

Having a neutral facilitator who was familiar with the maturity matrix and had creditability with Alliance members proved to be an important enabler of the testing process. The facilitator was able to assist with interpretation of the maturity matrix and move teams along if they got stuck on one element or indicator. The facilitator's credibility was important as this meant that Alliance leadership team members knew the facilitator, their experience in the health system and the history of their way of working. This knowledge and experience created trust with Alliance leadership teams and enabled them to assess their capacity and capability sincerely and not worry about presenting their better side or being judged on their discussions or results of their assessment.

When Buch, et al. [152] evaluated the use of the maturity matrix to develop quality improvement capacity and capability in Danish general practices, they reported that the use of a neutral facilitator, who understood the matrix and helped general practices with the self-assessment, 
positively influenced the implementation of improvements identified following the selfassessment.

Although senior managers from the $\mathrm{MoH}$ and the Health Quality and Safety Commission were involved in the construction of the maturity matrix, the role of these agencies in the future use of the maturity matrix is unclear. On the one hand, the MoH could use the results of the selfassessment process to understand local system conditions when commissioning new programmes or services. This understanding could inform the level of support and funding needed from the $\mathrm{MoH}$ to improve Alliances' capacity and capability and increase the chances of success with implementation of LST initiatives. On the other hand, involvement of the MoH may cause Alliance leadership teams to be less earnest in their assessment and hesitant in revealing their true capacity and capability with inadvertent or deliberate intent to present a better side of themselves. Given the central role of the $\mathrm{MoH}$ and the rich information it holds from a national perspective, it could also use the maturity matrix to provide its assessment on the capacity and capability of the Alliances. However, there is a real and perceived risk that information from the self-assessment process may be used as an instrument to demonstrate system accountability to government and/or to public. This will detract the maturity matrix from its original purpose of stimulating improvement within the health system and may become another compliance or 'tick box' activity for DHBs. A non-threatening approach would be to first engage all Alliances in using the maturity matrix to show the value of such a tool and that they would not be penalised if they assessed themselves to be at the beginning stages of the maturity scale. The MoH could support DHBs and Alliances to add a peer assessment component and sharing of assessment results, creating learning communities in which Alliances (or future locality networks) learn from and influence each other.

Alliances, health system leaders, and the $\mathrm{MoH}$ may also see this maturity matrix as an academic product that was constructed with a small group of people and tested with three alliance leadership teams. However, the DMIC model [162] was also based on a PhD research and on the premise of creating a tool to increase the awareness of co-ordinators, managers and professionals involved in the development of integrated care services and to improve their efforts and successes with integrated care. Following validation, the use of the DMIC tool expanded to other integrated care practices in the Netherlands.

Development of this maturity matrix is only the beginning. More work is required to test, improve and increase its fidelity, accessibility and adoption beyond the SLM programme and with minor 
modifications, beyond the New Zealand health system. The principles of Te Tiriti o Waitangi used in the maturity matrix would be relevant to engagement of most indigenous population in health systems world-wide [202].

The maturity matrix also applied most of the $\mathrm{CMO}$ groupings revealed in the research. For example, an alliancing way of working provides a platform for system leaders to practise distributed leadership, to build and maintain high-trust relationships, to agree shared vision and goals, and to involve local communities, iwi and health professionals in shared decision-making to achieve the desired system transformation.

When informal networks, such as Alliances and locality networks, reach and sustain the established or excellence maturity scale on the matrix across all key elements, there is an increased chance of success with implementation of LST initiatives and for these networks to achieve the desired system transformation.

The key local and national contextual factors are discussed in the next section. 


\subsection{Local contextual factors}

Findings from this research show that the history of working together and quality of relationships, distributed leadership from DHBs, and the maturity of Alliances are key local contexts that influence successful implementation of LST initiatives in the New Zealand health system.

The inability for Alliances to successfully implement the SLM programme depended on two factors: the command and control leadership style of DHB senior leaders because they did not believe in the alliancing way of working, which led to unequal power distribution resulting in a 'master-servant' relationship in the Alliance; or a lack of understanding of the role and opportunities with an alliancing way of working, which led to an underinvestment in the relationships and resources needed for the Alliance to deliver on its SLM plan.

The post implementation review of the SLM programme [121] identified that a transformation programme, such as the SLM programme, depended on the maturity of the Alliances. Those districts that had better collaborative relationships with strong Alliance structures and processes were more successful in implementing the SLM programme. The report concluded that maturity of Alliance was a pre-condition to enhance a culture of system integration and quality improvement.

While a requirement to form Alliances (or locality networks) can be mandated, the success of an alliancing way of working relies on a positive history of working together among alliance members. These contextual factors trigger the important mechanism of trust and strengthens relationships and drives behaviours and actions of Alliance members. One of the actions is the leadership style of DHB senior leaders. Presence of a distributed leadership style from these leaders sustains high-trust relationships between Alliance members because there is an equal distribution of power and Alliance members are willing to agree on a shared vision and goals for the district and commit to working collaboratively towards that vision (beyond their organisational and professional boundaries). Over time, the collaborative way of working creates a positive history of working together in which success is communicated to member organisations and failure is seen as an opportunity to learn from [1]. The positive history reinforces the distributed leadership style of the DHB senior leaders. Best, et al. [1] found presence of distributed leadership to be linked to sustained commitment to change efforts at the most senior levels of an organisation or system.

Conversely, a command and control leadership style leads to unequal distribution of power and a low-trust environment. Alliance members work in siloes representing their profession, 
organisation or community. There is no clear vision or goals or dedicated analytic or human resources. Alliance members may agree to actions but not implement either owing to potentially competing organisational priorities or a simple lack of commitment or willingness. There is no collective responsibility to the Alliance work programme. Members do not communicate the work programme of the Alliance within their organisations. The Alliance is not embedded in the DHB structure and does not have a clear mandate. DHB Boards and senior leaders see themselves as the sovereign organisation to make all the decisions about the design and delivery of health care in their districts. This disconnected way of working in a low-trust environment leads to negative history of working together, which enhances the chances of failure for Alliance to achieve its work programme. This failure to perform further reinforces the command and control leadership style from DHBs.

For an alliancing way of working, the key underlying mechanism of trust between Alliance members is sustained and nurtured by a continued commitment to distributed leadership from DHB senior leaders. The sustained trust strengthens a collaborative way of working, and if nurtured over time, creates a ripple effect that builds maturity of networks such as Alliances $[67,179]$. This maturity includes sustainability of relationships, research and solutions to address the big, 'wicked' health system problems and to adopt innovations to achieve system transformation [179]. This maturity could take many years to develop but also leads to Alliances being cohesive and resilient to the changing internal and external environment. The ripple effect of trust as a key mechanism to sustain an alliancing way of working was illustrated in Chapter 11, section 11.5 (Figure 11.13). Trust and a continuous commitment to power-sharing are regularly identified as realist mechanisms needed to successfully implement and sustain system transformation efforts $[67,179]$.

Other contextual factors, such as the capacity and capability for change and a continuous improvement culture, support the alliancing way of working. A continuous improvement culture encourages staff to challenge status quo and try new initiatives. It builds a thirst for self-critique and continuous learning and improvement. When system leaders recognise the importance of continuous improvement, they invest in the capacity and capability to enhance the organisation's readiness for change. The capacity and capability enable the provision of evidence that helps the system leaders to understand the variation in care, the root causes of inequities and their options to address these variations to deliver equitable care and improve health outcomes. When health system leaders experience the success of an alliancing way of working, they are empowered to 
think beyond their unit, department and organisation, and engage in whole-of-system improvement. Together, these contextual factors trigger mechanisms that strengthen the alliancing way of working with analytic support, human resources in the form of service level Alliance teams, and connection to other networks, such as consumer councils and quality improvement networks, to the Alliance structure.

These findings strongly resonate with evidence in the existing literature. Best, et al. [1] found that system leaders' commitment to continuous improvement and the quality of the information infrastructure and analytic capability as important contextual factors to establish feedback loops. Feedback loops allowed system actors to measure progress against LST initiatives through a blend of quantitative and qualitative measures and metrics $[1,3,30]$. A robust measurement framework instilled confidence in system leaders that a LST initiative is worth investing in and also avoid measures that will create perverse behaviours and incentives $[1,3,30]$.

Insights from the SLM programme and findings from the post-implementation review of the SLM programme [121] showed a large variation in the maturity of Alliances across New Zealand. This variation can be a constraining factor for successfully implementing LST initiatives. However, Plsek and Wilson [8] state that variation is a natural phenomenon of complex adaptive systems because of the local and national contexts and elimination of variation is futile and may stifle innovations. Instead a continuous learning approach using feedback loops is more suitable to increase Alliances' and health system leaders' understanding of programme architecture that underpins efforts to successfully implement LST initiatives resulting in an increased maturity. This approach is more likely to lead to a focus on interactions and relationships between system actors and pooling of resources to achieve the shared vision $[8,67,203,204]$.

\subsection{National contextual factors}

The national contextual factors, identified by research participants, that influenced successful implementation of LST initiatives related to system leadership, system accountability environment, and the 'new power' approach to design and implementation of LST initiatives. Some of these contextual factors are outside the control of and even influence of senior operational and clinical leaders (such as the DHB Chief Executives and the health care professionals) and have a significant influence on LST initiatives with a change in ideology or direction arising from changes in government, change in national accountability frameworks, or a change in key leadership roles such as the Director-General of Health. The national contextual 
factors also create tension between system accountability and improvement of system performance. This tension is further discussed in the following section.

\subsubsection{Health system leadership}

Distributed leadership emerged as a key contextual factor that builds high-trust relationships between system leaders. Research findings showed that distributed leadership from DHBs has a ripple effect in building and sustaining high-trust relationships between DHBs and system partners providing health care services in the district. At the national level, fostering collective leadership between the $\mathrm{MoH}$ and system partners providing health care services, such as DHBs, builds system leadership capacity and capability and facilitates collaborative learning.

Distributed leadership is aligned with a 'new power' approach where key leadership functions, which include making important decisions, are shared with a set of people and decisions emerge from a collective process through interactions of multiple actors $[1,13,35,196,197]$. In a distributed leadership style, leadership actions of an individual leader is less important than that of actions of a set of people $[1,197]$. Distributed leadership looks beyond leadership structures and functions and considers and acknowledges the contribution of all individuals and not just those in formal leadership or managerial roles $[196,205]$. Distributed leadership challenges 'new public management' ideas and shares a common theme with other leadership theories such as 'collective leadership', 'shared leadership', 'collaborative leadership', 'co-leadership' and 'emergent leadership' in which leadership is not the responsibility of just one person or those in formal roles but is a collective responsibility of actors in a system $[196,197,205]$. In complex adaptive systems such as health systems, distributed leadership brings 'new power' values that focus on achieving a shared vision by mobilising and leveraging actions of system actors and enabling continuous learning using feedback loops $[206,207]$. Change in health systems requires the buy-in, involvement and leadership of both operational and clinical leaders $[1,205]$. The latter usually do not hold formal leadership roles and are traditionally siloed in their area of speciality or profession but are critical in the success of any change effort. Distributed leadership provides a mechanism for clinical involvement and leadership, which is identified as a key element for successful implementation of LST initiatives in this research.

Boak, et al. [208] found six factors that enabled a distributed leadership style in health service improvement: a recognised problem that needs to be addressed; a relative narrow clinical focus for the change; active engagement of staff in the change process; a long deliberation and planning period; presence of standard operating procedures; and delegation of responsibilities 
and resources to support decision-making by teams. The study showed complexity of enacting a distributed leadership style at a micro service level. In LST initiatives that aim to bring change at a system level and that involve multiple organisations and system actors, the complexity of enacting a distributed leadership style increases exponentially. The use of trust-based networks like Alliances provides the platform for DHB senior leaders to demonstrate their commitment to distributed leadership.

Conversely, a command and control leadership style is more aligned with the 'old power' approach. It is a top-down leadership style where all important decisions are made by one or two individuals who are in formal leadership or managerial roles and hold the power that is inherent to those roles $[1,205]$. A command and control leadership style works within formal hierarchical organisation structures in which the leaders determine organisational goals, influence their staff to align and commit to these goals and lead them to achieve these goals effectively and efficiently [206].

The 'golden thread' concept in a Westminster-derived health system and the 'new public management' encourage a command and control leadership style to demonstrate accountability to government of the day and consumers of the system, and to meet the legislative requirements such as the Public Finance Act. The large collective deficit of DHBs exacerbates the use of this leadership style to manage their financial performance by the government and The Treasury through the $\mathrm{MoH}$. This transactional environment severely hinders implementation of LST initiatives, which relies on a distributed leadership model that supports collective action and continuous improvement.

Best, et al. [1] recommended a blend of a command and control and distributed leadership style for successfully implementing LST initiatives in complex adaptive systems. The researchers referred to this blended model as the alignment between top leadership and distributed leadership. According to the researchers, this could be achieved in four ways in practice. First, by creating an explicit alignment of shared vision and goals by top and middle managers so that actors at all levels in the system are clear on the strategic direction of the organisation. Second, through an active management of the change strategy using designated change managers so there are dedicated resources for managing change. Third, using small-scale projects to demonstrate to actors that change is possible and worthwhile, and actors are then more likely to be willing to attempt large change. Lastly, through providing an assurance that actors would not be penalised for attempting change efforts to achieve the change strategy. 
Uhl-Bien, et al. [206] argued that leadership in complex adaptive systems requires a blend of leadership styles that considers the unpredictable, emergent and generative nature of the system within the contexts of hierarchical and bureaucratic structures. The researchers recommend a blend of three leadership styles: (1) a traditional command and control style by those in formal managerial roles to fulfil the bureaucratic function of the system, referred to as administrative leadership; (2) an adaptive leadership style that mobilises the system actors to create knowledge and improve performance of the system through continuous learning; and (3) an enabling leadership to foster and create the conditions necessary for adaptive leadership to exist.

The researchers explained how these leadership styles work in practice. Administrative leadership refers to actors in formal managerial roles whose role is to plan tasks, recruit and manage staff, allocate resources, manage crises and conflicts, and co-ordinate activities to achieve the organisational outcomes efficiently and effectively. People are formally appointed to these roles and have relevant delegations depending on the hierarchy of the role. Adaptive leadership refers to adaptive, creative and learning actions that emerge from interactions of system agents as they respond to changes in their environment. People are not formally appointed to these roles. Adaptive leadership is informal emergent dynamic and is not an act of authority. Enabling leadership catalyses the conditions necessary for adaptive leadership to thrive and manages the tension between the bureaucratic (administrative) and the emergent (adaptive) functions of the organisation. According to Uhl-Bien, et al. [206], people in enabling leadership roles foster innovations and facilitate the flow of knowledge and innovative ideas from adaptive structures into administrative structures. This occurs at all levels of the organisation and depends on the hierarchical level of the role.

As commissioners of health services, $\mathrm{DHBs}$ and the $\mathrm{MoH}$ are responsible for meeting government priorities and legislative compliance but they are also responsible for continuously improving the performance of the system and delivery of health care that is patient-centred, holistic, coordinated and equitable. The collective evidence from findings of this research and evidence from literature assert that health system leadership cannot rely on one leadership style and that a blended model that considers the nature of complex adaptive systems is required to manage the dichotomy of system accountability and system performance. The blended model provides a balance of 'tight' and 'loose' approaches [209] for DHBs and the $\mathrm{MoH}$ to create collective 
leadership with their system partners to design and implement LST initiatives while demonstrating system accountability to their stakeholders.

\section{System stewardship}

As the central agency responsible for leadership of the health system, the $\mathrm{MoH}$ shapes the national policy environment that includes briefing the incoming Minister of Health, ongoing policy advice to the Minister of Health, implementing priorities and policies of the government, providing operational support to the health care providers (e.g. national data collections, analysis and reports) and demonstrating the value and confidence of system to the government and the public through appropriate accountability instruments [12]. The WHO refers this function as 'stewards of the health system' [210] and identified stewardship as one of the six (and the most complex) building blocks of health system strengthening to improve health outcomes [129].

In the first instance, the $\mathrm{MoH}$, as stewards of the health system, is responsible for improving the performance of the health system so that the system is able to deliver equitable health care and improve health outcomes. The $\mathrm{MoH}$ has an important role in outlining a system transformation strategy and creating conditions for collaboration to support the system with design, implementation, monitoring and evaluation of LST initiatives that contributes to the overall strategy. In saying that, the $\mathrm{MoH}$ has to be careful about how its role is operationalised, with its behaviours and interactions with other system actors, in that the support is not perceived as a command and control approach. The $\mathrm{MoH}$ (from the centre) can frame the strategy and facilitate its implementation, but experience with accountability frameworks showed that quality improvement cannot be driven from the centre.

However, the $\mathrm{MoH}$ is also responsible for demonstrating accountability and legislative compliance to system stakeholders that include the government, the Minister of Health, Office of the Auditor-General, The Treasury and the public [50]. These two spectrums of the stewardship role, system performance and system accountability, often cause tension in the system. The former needs a focus on system improvement through learning and innovation while the latter follows 'new public management' ideas and tries to control the system with rules and priorities set by the government of the day. Managing the two spectrums at a national level requires a balance between command and control and distributed styles of leadership to design and implement LST initiatives. 


\section{Health system leadership capacity and capability}

This research identified a need for a system leadership strategy or programme that strengthens system leadership capacity and capability.

Fawkes [205] undertook a review of literature and activity to provide the Department of Health in Victoria, Australia, with an innovative approach for developing leadership for disease prevention in the state. In her report, the reviewer distinguished between leader and leadership development: "the former emphasises human capital - individual attributes and capabilities - as a basis for increasing intrapersonal competence. The latter emphasises social capital - mutual obligations, commitments, trust and respect - as a basis for strengthening interpersonal competence" (p 44) [205].

The reviewer recommended that leadership development programmes needed to shift their focus away from correcting personal performance, skill and knowledge gaps to one that builds on existing knowledge and skills to enhance positive emotions and behaviours and invest in sustained learning strategies.

A flagship report by the Alliance for Health Research Policy and System Research (AHPSR) examined the different aspects of leadership to stimulate new thinking on leadership across the health system [211]. The report highlighted that building leadership capacity required participation from all system actors working together as a single group of leaders to challenge and support each other and foster collective leadership to advance the long-term strategy of the health system. The report called for "leadership at multiple locales and levels, which is inclusive, collaborative, open minded, adaptive, persuasive and receptive" (p 29).

Given the impact of wider social determinants of health on health systems, health system leaders also need to engage with other social sector agencies to influence their policy leadership and implementation. A collective leadership model provides stability and resilience to changes in senior leadership roles and emergencies created by pandemic and natural disasters [211].

Other studies have expressed similar sentiments that collective strength builds system leadership capacity and capability and improves system performance [72,212]. Collective leadership requires a platform for system actors to engage and participate. Forums such as think-tanks and learning communities at national and local levels provide platforms for collective leadership to build and sustain system leadership. 
With the 'Better, Sooner, More Convenient' (BSMC) business cases, the MoH provided seed funding for PHOs to form Alliances with DHBs. Some of this funding was used to make available independent facilitators, who were senior and credible system leaders, to 'broker' relationships between DHBs and PHOs. This model was repeated with the national health targets programme where each health target had a clinical champion from the sector who was responsible for working with the system partners at the DHB level to improve DHB and PHO performance against the target.

During implementation of the SLM programme, facilitating relationships between DHBs and PHOs was an important part of programme manager and clinical lead roles. This was especially important in districts where there was a poor history of collaboration or low-trust relationships among senior system leaders. Similar observations were made during testing of the maturity matrix, where having an independent facilitator kept the discussion focused on the task and managing conversations at the whole-of-system level.

Experience from the BSMC, national health target, and the SLM programmes along with findings from this research suggest that it is necessary to build national learning communities such as think-tanks, communities of practice and a network of independent facilitators. These learning communities can support networks such as Alliances with successful implementation of LST initiatives, building capacity and capability for improvement, and 'broker' local relationships. Best and Saul [212] said that "this type of activity supports progress not only in evaluation and learning, but also country ownership, engagement, human resource capacity, power and integration" (p 23). Best and Saul [212] went on to say that learning communities encourage partnerships between system partners to pool in limited resources available in the system, especially for small to mid-sized organisations, who do not have the same capacity and capability as some of the larger ones. By supporting, facilitating and funding learning communities, the $\mathrm{MoH}$, as the system steward, creates conditions for collaboration and system transformation [72].

Findings from this research showed that another way to build leadership capacity and capability is to work with professional organisations and education providers to include change management and leadership skills as part of all health care training programmes. This will lead to the health care professionals being aware of the complexities of the health system from the beginning of their career and understanding that providing a high-quality health care involves coordinated care from across the system and not just the patient in front of them or their speciality. 
It will help them understand the system dynamics and constraints that contribute to funding and professional practice decisions and how to navigate the system to improve the quality of care [1]. Health care professionals will become change agents in the system.

\subsubsection{A learning system}

Participants in this research identified a lack of long-term planning in the New Zealand health system and a lack of a national transformation strategy that provides direction to system actors for investment and LST initiatives. Research participants said that instead of managing and controlling the system, system leaders need to guide the system as a whole rather than managing individual organisations. One of the participants used the analogy of a flock of birds and said that a change in direction cannot be achieved by controlling individual birds, but rather guiding the direction of the whole flock.

There was a desire for a long-term plan from those working in the health system to build resilience against the three-year political changes and changes in key leadership roles such as the Director General of Health. The Health and Disability System Review recommended development of a 20-year health plan, along with five-year regional plans to supplement the annual district plans [23].

The New Zealand Health Strategy refreshed in 2016 provided a national transformation agenda [115]. The strategy was accompanied by an action plan, however, with the change of government and the Director General of Health, the strategy lost its visibility and the focus for implementation and monitoring. This is not an uncommon phenomenon in a complex adaptive system where changes in key leadership roles undermine system leadership capacity and disrupt the direction of travel for the system [212].

The New Zealand health system does not lack plans to guide the improvement of system performance - long-term strategic plans, such as the Primary Health Care Strategy, He Korowai Oranga, and the New Zealand Health Strategy have existed since the early 2000s, as well as recently developed plans, such as Whakamaua. As part of the upcoming reforms, there will most likely be a new long-term national health plan, medium-term regional health plans, and annual district plans. The gap has been coherent implementation of the plans and monitoring progress to ensure these are achieving the envisaged vision and goals.

Further, much contemporary scholarship about health system change reinforces the relational and adaptive way change occurs and the ability for the system leaders to recognise, support, scale-up and spread changes that have the potential to cause change at the system-level 
$[70,194,198]$. System leaders need to embrace the power of health systems that comes from the system-level adaptation that emerges from micro actions of system actors (the 'flocking' effect discussed in section 4.1).

The emerging 'Human Learning Systems' (HLS) offers a framework that bridges the gap between the theory and the practice of system transformation and emphasises the relational and adaptive way of working to achieve the desired transformation of the system [213].

The HLS consists of three dimensions [213]. First, the human dimension that incorporates a relational aspect, both to involve whānau and communities to design services, and having faith in health care professionals to act with human compassion and care. This dimension emphasises the need to be 'more human' and enables the design and delivery of patient-centric health services.

The second dimension focuses on continuous learning to overcome the culture of blame and a sole focus on accountability. In complex adaptive systems, learning is a key engine of service improvement and occur in many forms: appreciative inquiry, reflective practice, learning communities, learning partnerships, and rapid learning cycles. In practice, continuous learning means taking an iterative approach to working with people, shifting commissioning of services to funding organisations' capacity to learn, using data for monitoring and reflection rather than achieving targets, and creating a learning culture in which people feel safe to share their failures as well as successes.

Finally, the third dimension is having a systems mind-set, focusing on building high-trust relationships between system actors, establishing shared vision and goals, and shared values that guide behaviours. This dimension shifts the focus away from organisations to social interventions.

The findings from this research resonate strongly with the HLS concept and the use of the HLS concept helps situate these findings in the emerging health system transformation literature. Table 12.2 shows how the key elements identified in this research link to the three HLS dimensions. Further, the self-assessment maturity matrix operationalises the three HLS dimensions and supports the notion of a learning system for the pursuit of LST initiatives. It does so by facilitating deliberate, conscious and continuous learning of health system leaders and use of informal networks that bring all agents in the system together to solve complex problems that cannot be solved individually. The system leaders and networks acquire new information through the continuous self-assessment process, which changes their understanding, their interactions 
with other system agents, and their understanding of the contextual factors that enable and constrain implementation of LST initiatives. The continuous self-assessment process creates feedback loops that is critical to support the notion of a learning system [70].

\begin{tabular}{|c|c|}
\hline HLS dimension & Key elements from this research \\
\hline Human & $\begin{array}{l}\text { Clinical leadership and involvement } \\
\text { Involved people, whanau and community }\end{array}$ \\
\hline Continuous learning & $\begin{array}{l}\text { Intelligent commissioning } \\
\text { Continuous improvement } \\
\text { Analytic capability } \\
\text { Dedicated resources and time } \\
\text { Understanding of equity } \\
\text { Integrated health information }\end{array}$ \\
\hline Systems mind-set & $\begin{array}{l}\text { Alliancing way of working } \\
\text { Commitment to Te Tiriti o Waitangi }\end{array}$ \\
\hline
\end{tabular}

Table 12.2: Key elements mapped to Human Learning Systems Dimensions

According to Holmes, et al. [70], a learning system cannot solely rely on simple and sequential actions that assume a high degree of rationality and linearity in which knowledge is produced, disseminated to end users and then passively incorporated into practice and policy. Instead, an active transfer of knowledge or knowledge-to-action in complex adaptive systems involves coproducing knowledge, establishing shared goals and measures, enabling central and distributed leadership, and ensuring adequate resourcing $[70,194,198]$. In practice, this means support from senior system leaders for change efforts, a strong narrative to convince frontline staff, involving frontline staff in the change efforts, and creating and maintaining an organisational culture that enables prototyping small-scale innovations and then refining these through rapid learning cycles $[70,194,198]$. The maturity matrix constructed in this research offers the opportunity for an active transfer of knowledge or knowledge-to-action for successful implementation of LST initiatives.

While a long-term plan may be necessary to provide the government policy direction, this plan is likely to change with changes in the political environment. Therefore, the focus needs to be on building the capacity and capability for health system transformation to adapt to the government policy and narrative of the day (to guide the direction of the flock). The HLS concept encompassing findings of this research provides the framework to build the system transformation capacity and capability and support the notion of a learning system. 


\subsubsection{The 'new power' approach to design and implementation of LST initiatives}

Ansell, et al. [201] highlighted the barriers or stumbling blocks to successfully implementing national policies because of the dichotomy of system accountability and system performance. They argued that this dichotomy separated policy design from policy implementation. The national policy design follows 'new public management' doctrines with a top-driven approach and is usually political, and implementation by system agents is expected with the institutional and hierarchical arrangements in the health system. However, implementation of these policies often fail because of a lack of buy-in from frontline staff on the proposed solutions, method of implementation, disruption of work practices of frontline staff, expectations of patients and communities, and illogical, passive resistance from frontline staff who have not been involved in the policy design [201]. This experience is commonly documented in much of health policy literature [35].

Ansell, et al. [201] recommended collaborative policy design where policies "can be more effectively designed by connecting actors vertically and horizontally in a process of collaboration and joint deliberation" ( $p$ 475). They did not presume that collaboration will be unanimous consent but argued that it will aim "to harness difference without eliminating it" (p 475) [201]. Further, the researchers recommended adaptive implementation that recognises local contexts and flexibility for subsequent changes in policy and its implementation approach with insights gained through the ongoing implementation process. Adaptive implementation enables continuous improvement in a learning system.

The SLM programme is an example of a national policy that was collaboratively designed and implemented through Alliances using a continuous improvement approach. My research findings confirmed that the collaborative design and adaptive implementation increased engagement of system actors, in particular frontline staff, in the implementation of the SLM programme and made them feel more confident to be innovative and do things differently, which led to sustained commitment to the programme. Two other New Zealand examples of 'new power' approaches are the Healthy Families NZ initiative [214] and the Whānau Ora commissioning programme [215].

Healthy Families NZ is a LST initiative implemented in 10 different geographic communities across New Zealand to prevent chronic disease using community leadership [214]. The initiative aims to identify, design and implement changes to enable people to make healthier choices and live healthier lives by being physically active, more people smoke-free and reduce alcohol-related 
harm. The lead providers of the programme include Māori and iwi organisations, Regional Sports Trust promoting physical activities, and Local Councils. One of the key investment areas for the programme is activating local leadership groups. Healthy Families NZ teams work collaboratively with local leaders and lead providers to influence transformational change [214].

The five building blocks of the programme are workforce, leadership, relationships and networks, resources and knowledge and data [141]. The $\mathrm{MoH}$ approach is placed-based, explicitly taking into consideration the context of complexity in which the initiative was being implemented and uses te ao Māori lens to improve Māori health [214]. An evaluation of the initiative revealed that the collaborative approach, activation of local leadership and informal networks, and empowering of local teams led to local ownership of the programme and strengthened community trust to achieve the aims of the initiative [141].

Whānau Ora is a unique public sector initiative that devolves delivery of Whānau Ora services to community-based commissioning agencies. These agencies work in partnership with providers and navigators to deliver customised services to improve wellbeing of individuals and whanau, empowering them to become self-managing and achieve their aspirations [215]. Whānau Ora encompasses services such as health, education, housing, employment and income levels. Whānau Ora providers are embedded in the communities they support and use local knowledge to understand the needs of communities, adapt ideas, and think of innovative solutions that is relevant for each community. The navigators are specialist staff who advocate for whanau and work with whanau to find services and support needed [215]. The programme is underpinned by strong networks and relationships with providers and whanau.

There are similar international examples of a 'new power' approach.

The Saskatchewan health system in Canada used hoshin kanri, as part of an overall Lean Management approach, to develop their health system plan to improve the structure, culture and quality of health services [216]. Hoshin kanri is a method of strategic planning that emphasises the importance of whole system engagement that harnesses skills and experience of all employees in the development of strategies, targets and actions [216]. Willis, et al. [216] reported an unprecedented level of involvement from health system leaders and communities in the development of their health system plan. This approach ensured a plan informed by those who work in the system and understand the complexity of the system, the challenges in the system, and the need for iterative learning to transform the system [216]. 
A white paper developed by NHS Improving Quality presented four case studies that used a 'new power' approach for transformational change in the United Kingdom [35]. Success in the transformation efforts across these four case studies was owing to networking to develop relationships, use of social media to engage people and create a movement for change, creation of peer support and multidisciplinary social groups, involving frontline staff and patients, focussing on a shared purpose, building relational skills, working in networks in a non-hierarchical way, creating communities of change agents who learn and share lessons and their experiences [35]. The paper asserted that organisations that use 'new power' approach of "building shared purpose, connectivity, imagination, relationships and empathy" ( $p 19)$ are more likely to achieve their transformation agenda and deliver changes than those solely using the 'old power' approach [35].

\subsubsection{System accountability environment}

System accountability or organisational reform were not identified in this research as key elements to support system transformation to deliver people-centred, holistic and equitable health care. However, the system accountability environment was identified as a national context that influenced the successful implementation of LST initiatives. The accountability environment included frameworks and instruments the MoH used with DHBs to achieve financial accountability (DHB surplus/deficit), performance accountability (service outputs and targets), and political accountability (good use of public money).

The $\mathrm{MoH}$ uses a small set of financial and non-financial accountability measures with national targets as a proxy for DHB (and system) performance. Evidence suggests that this approach skews the accountability perspective because system leaders at DHBs and their Boards focus on achieving these measures at the expense of the broader population health outcomes [217]. This was particularly evident with the emergency department national health target $[89,90]$ and with the continued focus on the financial performance of DHBs. Participants stated that the current national template approach to accountability by the $\mathrm{MoH}$ does not take into consideration local contextual factors and drives passive compliance ('tick box' exercise) by health system leaders, defiance by clinical leaders, and perverse behaviours by some to 'stay in the middle of the pack' to avoid sanctions and penalties.

While the Interim Report of the Health and Disability System Review [23] highlighted the undesirable consequences of 'new public management' doctrines, the final report focused strongly on organisational reforms and strengthening existing system accountability instruments 
to achieve the transformation required to deliver equitable health outcomes and to become a more financially sustainable system. The Interim and the Final Reports of the Health and Disability System Review failed to acknowledge the rise of 'new public management' that followed the organisational reforms of the 1990s, which led to a fragmented approach to planning and delivery of health care and transactional relationships [80].

Greenhalgh and Papoutsi [6] state that the emergent, unpredictable and generative nature of the health system requires a paradigm shift from a narrow linear focus of cause-and-effect from single intervention to "rich theorising, generative learning and practical adaptation to changing contexts" ( $p$ 1). They argue that "quality and safety improvement can be achieved by 'attending to' rather than trying to 'control for' complexity" ( $p 3)$. This involves moving away from the current thinking of the health system as a machine and trying to control each part in isolation with tight accountability instruments [8]. Instead of national service or accountability frameworks and detailed service specifications, Plsek and Wilson [8] recommended minimum specifications. Minimum specifications allow an environment for innovation, shared action, and facilitate interactions and relationships by providing direction, boundaries, resources, and permissions $[8,218]$. The participants in this research also made a similar recommendation, which they referred to as 'safety margins' of performance.

Findings from this research suggest that the current accountability environment hinders successful implementation of LST initiatives because according to research participants, the system leaders at DHBs and their Boards place more effort on achieving accountability measures to avoid sanctions and penalties.

Further, a senior Māori clinical leader said that system actors feel disempowered to engage with their local communities, Māori and Iwi, and therefore fail to recognise patients and communities as customers of the system. The system leaders are also seen to fail to meet their Te Tiriti obligations, in particular, the principles of partnership, Tino rangatiratanga, active protection, and supporting expressions of hauora Māori models of care. These principles in practice mean that Tangata Whenua would decide what system transformation initiatives are needed for Māori, the vision and goals of these initiatives, and how these are implemented and monitored (and not the 'golden thread' approach from the government) [101].

Steele Gray, et al. [218] studied organisational responses to accountability requirements from home and community care agencies operating in Ontario, Canada. The study found that organisational responses to government-imposed accountability requirements took many forms. 
These included: passive compliance by simply meeting accountability requirements; active compliance through internal changes, such as subcontracting or partnerships; and noncompliance through defiance or avoidance. The study recommended that compliance with accountability requirements can be improved if those imposing took into consideration external factors that may influence organisational responses and tailoring agreements and supporting organisations in their efforts to comply.

In Australia, the Victorian Health Services Performance Monitoring Framework [219] was designed as a risk-based framework with more intensive support and monitoring for those services and organisations with greater risks to the safety, accessibility and sustainability of services, and with a stronger emphasis on contextual factors that underpin performance. The contextual factors include financial management, governance and leadership, quality and safety indicators, and qualitative measures that would indicate performance issues ahead of performance failures. The goal of the framework is for the Victoria Department of Health and Human Services to work collaboratively with Victorian health services and other stakeholders to: identify performance risks; analyse performance; assess performance outcomes; and address performance risk, undertake monitoring and provide support [219]. Such an accountability framework promotes transparency, shared accountability, and facilitates relationships and interactions for continuous performance improvement across the system [8,218,219].

\subsubsection{Funding environment}

The funding environment was identified as a contextual factor that both enabled and constrained successful implementation of LST initiatives. Funding and commissioning play a critical role in system transformation, for example, promoting a collaborative way of working rather than a competition. Research participants said that organisations with large funding dominated conversations relating to the commissioning of services and smaller organisations, such as NGOs, were limited in their ability to influence change owing to the competitive commissioning environment.

There is no ideal model to fund delivery of health care services and even if there was one, it would not work for all as it will be affected by local contextual factors such as the system design and the population demographics. There is also not an ideal level of funding that could meet current and future demands of health systems as evidence suggests that the funding level alone is not responsible for health inequities or the DHB deficits in the New Zealand health system [23]. 
Sometimes a lack of or constraint on funding provides the burning platform for transformational change. At the time of writing this thesis, the world was amid a global pandemic with COVID-19 response stretching the resources of health systems worldwide. In New Zealand, there were reports of unprecedented levels of cooperation among funders and those responsible for delivery of health care and system transformations that occurred in a short space of time to enable the health system to effectively respond to COVID-19. Examples of these included a transformational change in the model of care delivery with general practices' rapid shift to teleconsults and the use of e-prescription technology for medicine dispensing with 48 hours of notice before the country went into lockdown. Furthermore, there were great advances in the use of technology to connect regional public health units to a national contact tracing database, central distribution of personal protection equipment and influenza vaccines, and the development of a contact tracing mobile application that had inter-operability with other health information systems. Although funding was provided for these changes, often funding followed the change and it was the pandemic response that had provided the burning platform and the unprecedented level of co-operation that had achieved the transformations.

The New Zealand 2020 Budget, which was delivered in the middle of the pandemic, included significant investment for the health system. This record level of investment was to recognise the historical systemic underfunding, the additional surgeries and treatments needed to clear the backlog owing to COVID-19 response, and the need to re-build the health system to meet current and future demands [220]. While most in the system welcomed this investment, some were apprehensive about whether the significant additional funding was going to reach the right parts of the system to improve health outcomes for Māori and other high priority populations $[221,222]$. Some went further to say that the investment in hospitals perpetuated the diseasefocused, reactive model of care instead of investing in the primary and community services to build on the LST initiatives that resulted from the COVID-19 response $[223,224]$.

Research participants and the literature $[3,30,70,122,141,194,200]$ are both strong on the need for dedicated resources for LST initiatives, both in the form of new resources and re-alignment or re-prioritisation and upskilling of current resources to match the needs. Resources needed for successful implementation of LST initiatives include human resources in the form of appropriate sustainable health workforce, project or programme managers, information technology capability, change management leadership and evaluation skills, and enough time for change to occur [70]. Except for health workforce, senior health system and political leaders often 
underestimate the importance of these resources. These are often perceived as administrative functions, which are 'nice to have' but not essential for a high performing health system. This perception needs to change for the health system to be successful with implementation of LST initiatives that will achieve the desired system transformation. 


\subsection{RECOMMENDATIONS}

The New Zealand health system is at a pivotal point given the recent release of the Health and Disability System Review report. The recommendations in the final report, if implemented, will bring significant organisational reforms to the health system. There is a risk that powerful groups such as professional bodies and PHOs may resist the organisational reforms and these tensions will further affect the relationships, trust and commitment needed among health system leaders and organisations to deliver patient-centred, holistic and equitable care. Organisational reforms tend to make organisations and individuals focus inwardly to protect their roles, organisations, funding and place in the system. This research has identified conditions critical to support LST initiatives that goes beyond organisational and professional boundaries. Change in complex adaptive systems requires mobilisation of all system actors to work towards a shared vision and take into consideration behaviours and interactions between system actors and the social and cultural contexts that exist in the system. The recommendations below do not intend to undermine the organisational reforms that will inevitably follow from the Health and Disability System Review. These recommendations are made to strengthen the transformation that the New Zealand health system and health systems world-wide are seeking to achieve equitable health outcomes and a system that is strong and financially sustainable.

\subsection{Recommendation 1: A collaborative way of working}

This research findings show that informal trust-based networks are an important feature of complex adaptive systems and that these networks are critical to successfully implement LST initiatives. In the New Zealand health system, Alliances are used as an informal trust-based network to bring all system partners together to work towards a shared vision. The current policy environment requires DHBs to form an Alliance with PHOs providing primary health care in their districts. This environment may change as recommendations of the Health and Disability System Review are implemented and Alliances are replaced with locality networks. Regardless of what these networks are called, it is important that health system leaders understand the ripple effect of the key mechanism of trust that is triggered in the presence of distributed leadership that leads to an agreement of a shared vision and a commitment to work towards that vision. I recommend that health system transformation teams use findings of this research, in particular the self-assessment maturity matrix, to understand the inner workings of trust-based networks and the capacity and capability that is needed to support such networks. The maturity matrix and the $\mathrm{CMO}$ theories reported in this research provide valuable information for health systems 
world-wide on the programme architecture that underpins efforts to successfully implement LST initiatives using a collaborative way of working.

\subsection{Recommendation 2: The self-assessment maturity matrix}

One of the gaps identified through the implementation of the SLM programme was the lack of a practical tool that describes the key elements needed to support successful implementation of LST initiatives and the indicators of success. Such a tool would enable networks, such as Alliances or locality networks, assess their maturity on their capacity and capability to successfully implement LST initiatives. The maturity matrix constructed with health system leaders in this research bridges this gap and provides the beginning of a national tool to consciously and deliberately support learning of networks, such as Alliances and locality networks. The use of the maturity matrix generates feedback loops that stimulates learning and is more likely to lead to shift in behaviours among system actors. I strongly recommend that the Transition Unit leading New Zealand health system reforms uses the self-assessment maturity matrix developed in this research to prototype the locality-based networks signalled in the reform. Health systems worldwide could use the maturity matrix to assess and build capacity and capability needed for system transformation and to support a system that is continuously learning.

\subsection{Recommendation 3: A national improvement team}

This research identified the important role of system leadership and continuous learning in complex adaptive systems, such as health systems, to improve the performance of the system. Participants identified both a lack system leadership capacity and capability currently in the New Zealand health system and the need to strengthen it. Taking into consideration the evidence from literature and my research findings, I recommend that the $\mathrm{MoH}$, as the steward of the system, convenes a national improvement team that is underpinned by the three dimensions of the 'Human Learning Systems' concept; human, continuous learning, and systems mind set.

A national improvement team may comprise perspectives of senior operational and clinical leaders, Māori and Iwi, consumer advocates and the research community. It would support and facilitate continuous learning efforts, nurture relationships to build and maintain trust between system actors and build system leadership capacity and capability. This team could support the $\mathrm{MoH}$ to fulfil its functions as the steward of the system and will give effect to the national charter recommended by the Health and Disability System Review. The membership of this team has to be purposefully recruited to ensure that right people are selected who will work beyond their organisational and professional boundaries in a high-trust environment with a commitment to 
achieve the goals of the system. This way of working adds 'new power' values and will create learning community at a national level that will harness energy through collaboration, peer coordination and influence, and will share lessons from implementation of LST initiatives. The members could also act as facilitators or 'relationship brokers' to develop and enhance the preconditions required to build maturity of local trust-based networks such as Alliances or locality networks. Overall, this team could provide a powerful instrument for fostering and strengthening system leadership capacity and capability. At the national level, the MoH could work with the Health Quality and Safety Commission (HQSC) to co-ordinate opportunities for collaborative learning. Owing to scarce resources, business continuity and impacts of COVID-19, it is challenging to bring staff and leaders physically together frequently. However, with advances in technology and the unique experience of a virtual way of working during lockdowns, the $\mathrm{MoH}$ and the HQSC could explore more sustainable ways of creating online or virtual learning communities. The role of central agencies, such as the $\mathrm{MoH}$, is to co-ordinate such a network to manage expectations of system partners, to use the network to design and implement national policies, and to maintain focus on the long-term vision and goals of the system.

\subsection{Recommendation 4: A national improvement budget}

This research identified dedicated resources as a key element to support successful implementation of LST initiatives. The incentive funding attached to the SLM programme for PHOs created tension in the system with the responsibility of the programme implementation sitting with DHBs. The incentive funding for PHOs provided a strong incentive for PHOs to be involved in the development and implementation of the SLM programme. The funding, albeit small (\$5.33 per enrolled patient) [63] compared to the overall primary care budget, acted as a strong incentive for operational and clinical leaders from PHOs to come to the table. However, the funding also created an unnecessary distraction for the programme in that the programme was seen as a primary care performance programme instead of a whole-of-system improvement programme.

Findings from this research support that successful implementation of LST initiatives require dedicated resources in the form of funding to employ programme or project managers with change management skills, locum support to allow frontline health care professionals to participate in the change programme and appropriate investment in technology and analytic capability. It is therefore recommended that the $\mathrm{MoH}$ set a national improvement budget to support implementation of the national transformation initiatives. The improvement budget 
could be held and managed by the $\mathrm{MoH}$. The national improvement team would advise the $\mathrm{MoH}$ on distribution of the fund. A national improvement budget takes the tension away from incentivising health providers separately and ensures that LST initiatives that are funded contribute to the overall health system strategy and that the funding is systemic and sustainable.

\subsection{Recommendation 5: A change in the system accountability environment}

My research findings suggest the current DHB accountability framework is not suitable to support a learning system. Instead of a national template-driven framework that focuses on achieving national targets on a small set of financial and performance measures, a more sophisticated and supportive framework is needed that takes into consideration local contexts in which DHBs are operating. My research-based insights recommend a national accountability framework comprising three dimensions: smart monitoring, system support, and feedback loops.

Smart monitoring involves using insights from data, and monitoring reports and planning documents, such as the DHB annual plans and the SLM plans, to determine DHB performance against national minimum specifications.

The system support dimension involves the national monitoring agency (whether it is $\mathrm{MoH}$ or the proposed new Health New Zealand), who would develop national minimum specifications for safe, effective, efficient and equitable delivery of health services, and monitor DHB performance against those using smart monitoring tools. The Final Report of the Health and Disability System Review [23] signalled the minimum specifications for tier one services. The national monitoring agency would tailor improvement focus areas for each DHB based on evidence from past performance and their local contexts, such as the demographic profile and service configurations. The national agency's role would be to develop internal capabilities, tools and strategies to diagnose performance issues and respond rapidly where DHBs are not meeting the minimum specifications. This response would be determined using the feedback loops dimension and will involve different approaches depending on the contexts in which the DHB is operating.

The feedback loops dimension connects the smart monitoring dimension to the system support dimension and drills into the underperformance of a DHB. For example, if a DHB with a high Māori population is not meeting its financial targets because they are attempting LST initiatives that are designed by their communities, the response would be supportive and would seek to understand the DHB approach, assess the DHB's capability for implementing LST initiatives and provide support where DHB capability is lacking. In contrast, if a DHB is failing to address health 
inequities and is not involving their communities in the design of health services, then the response would focus on performance management and seek to understand if the DHB has capability to improve its performance and if not, what support it needs to improve its performance.

Figure 13.1 illustrates my recommendation. A more flexible and supportive framework, such as this, would enable DHBs to involve their communities in the design and implementation of LST initiatives. It would enable DHBs to focus more broadly on improving performance in areas they are underperforming, where health inequities may exist or improvement areas deemed important by their communities, rather than solely placing their efforts on achieving national targets for a narrow set of accountability measures deemed important by the government.

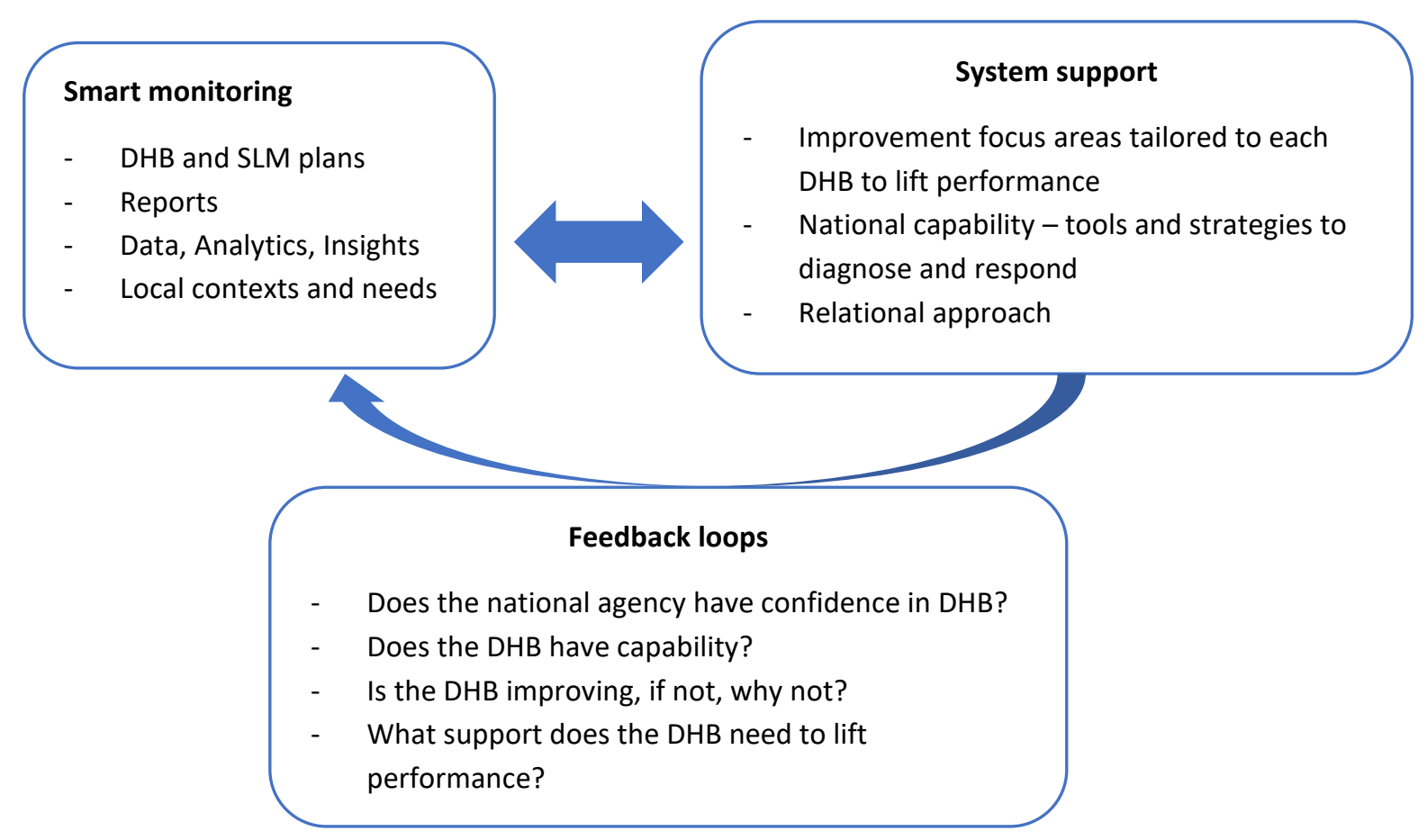

Figure 13.1: New proposed national accountability framework 


\subsection{STRENGTHS AND LIMITATIONS OF THE RESEARCH}

\subsection{Strengths}

A key strength of this research was my prior knowledge of the New Zealand health system, the key actors in it, and the direct experience with leading the national implementation of the SLM programme. The inside knowledge of the health system, three years of first-hand experience and knowledge were invaluable to the research. This knowledge and experience gave me the leverage to invite participants from across the health system who were involved in the SLM programme implementation and those considered leaders within systems thinking and LST initiatives. Prior knowledge about the functionality and make-up of the Alliance leadership teams was valuable in the selection process for field testing of the maturity matrix. I was able to invite three teams that had differed in their membership, size of the district they served, and the state of their local relationships.

The wide range of perspectives added to the rigour of the research and provided a unique wholeof-system approach to the research. It also assisted with managing (unconscious) bias that the SLM programme clinical lead and I brought to the research. This rigour gives me confidence that the findings of this research will not only be accepted by the sector but will also make a valuable contribution towards implementation of future LST initiatives.

\subsubsection{Health participants}

The research involved senior system leaders from the New Zealand health system. More than 100 people from across the New Zealand health system contributed to this research through interviews, workshops, online survey and testing of the maturity matrix.

The New Zealand participants included DHB and PHO staff that were involved in the development and implementation of the SLM programme. Given the life and a system-wide implementation of the SLM programme, there was a large sample pool from which participants in the study were selected. The multiple methods of data collection allowed me to involve participants from different levels ranging from senior operational and clinical leaders to frontline staff. Participants in the workshops included General Managers from the DHB Planning and Funding units, PHO Chief Executives, senior clinical leaders, and senior leaders from the $\mathrm{MoH}$ and the Health Quality and Safety Commission. The online survey was used to collect data from the frontline staff involved in the implementation of the SLM programme. The field testing of the maturity matrix included senior operational and clinical leaders from DHBs, PHOs and other health system partners such as Māori health providers, pharmacy, the Accident Compensation Corporation, and 
allied health leaders. Interviews were used to collect data from those in the research and health consultancy communities. The overseas participants provided an international view that supplemented evidence from literature on health system reforms, leadership and complexity thinking. The small size of New Zealand, my inside knowledge of the system and my existing relationships presented the advantage for participants from across the whole system (horizontally and vertically) from senior leaders to frontline staff to be involved in the research.

\subsubsection{Non-health participants}

The non-health interview participants were from a large New Zealand bank $(n=1)$, a construction company $(n=1)$ and a strategic organisational management consultant $(n=1)$. Braithwaite [11] argues that no other system is more complex than the health system and that no other industry or sector has a similar range and breadth of complexities that involve multiple providers planning, funding and delivering services to clients with diverse needs with many health care interventions personalised to suit individual needs. Additionally, the regulatory environment that the health system works in is uneven with some parts tightly controlled such as pharmaceuticals and others driven solely by individuals in the system such as diagnostic and treatment decisions by the health care professionals. The health system has multiple stakeholders and the interactions between these, the activities, and outcomes are infinite [11]. I agree with Braithwaite's argument but am also aware of many efforts to learn from industries to improve the efficiency and performance of the health system; for example, the alliancing way of working from the construction industry [68]. True to a realist sampling strategy, I wanted to test my theories with a small number of participants to seek an 'outside' perspective from those with knowledge and experience of leading implementation of LST initiatives in non-health industries to determine if there were elements and contextual factors that were common across complex systems.

\subsubsection{Te Tiriti o Waitangi}

A realist approach, nested within the framing of complex adaptive systems, enables the research to respond to social and political conditions relating to indigenous health [78]. Equity, partnership, participation, active protection and self-determination, which are principles of Te Tiriti are also fundamental to any LST initiative and is at the core of the 'new power' approach. As such principles of Te Tiriti were considered in the research in many ways.

Māori view was actively sought through the selection of research participants to ensure all workshop participants had equity expertise, especially for Māori. Additionally, Māori health system leaders were purposefully selected in workshops and interviews to provide Te Tiriti 
perspective. This selection approach could not be assured for the online survey, which was anonymous, or with Alliance leadership teams involved in the testing of the maturity matrix as these were existing teams with membership determined locally. However, to mitigate this, Māori equity and Te Tiriti perspective on the maturity matrix was sought and provided by the $\mathrm{MoH}$ Māori health Strategy and Policy team.

Te Tiriti and equity formed part of the programme architecture that underpins efforts to successfully implement LST initiatives, both vertically and horizontally. A commitment to Te Tiriti and understanding of equity were identified as two key elements that need to be present to increase the chances of success with implementation of LST initiatives in the New Zealand health system. Further, the alliancing way of working, and involved people, whānau and community elements foster participation of hapū, whānau, Iwi and Māori in the governance, design and delivery of health care and LST initiatives. These elements ensured that Te Tiriti and equity were vertically embedded in this architecture.

A successful outcome for the research would be an enhanced understanding of and a stronger focus by health system leaders and Alliances on reducing health inequities for Māori in their districts through successful implementation of LST initiatives. The maturity matrix identified two indicators for each of the 10 elements to horizontally embed equity and Te Tiriti in the maturity matrix.

Overall, the programme architecture constructed for successful implementation of LST initiatives recognised Te Tiriti as an important context for the New Zealand health system. The maturity matrix translated principles of Te Tiriti into practical actions that allows Alliances to assess where they are on the maturity scale for equity and Te Tiriti indicators and identify areas for improvement. Empirical work showed that this self-assessment process would enhance the understanding of health system leaders and Alliances about the programme architecture that underpins efforts to successfully implement LST initiatives to address health inequities for Māori and other high priority populations.

The number of Māori participating in this research was low, which is why input from the $\mathrm{MoH}$ Māori Health Strategy and Policy team was sought on the maturity matrix. A wider range and a higher number of Māori participants may have better reflected Māori aspirations in the maturity matrix. 


\subsubsection{The realist approach}

A realist logic of enquiry was well suited to inform my research questions and is a strength of this research. It unpacked and revealed the underlying, unseen mechanisms that caused variations in districts' ability to successfully implement the SLM programme. Most importantly, it enabled me to form the CMO theories that influence successful implementation of LST initiatives in different contexts. These $\mathrm{CMO}$ theories form an important part of the programme architecture that bridges the gap between the theory of LST initiatives and the reality of successfully implementing these in the New Zealand health system.

However, this research was my first attempt to undertake a realist inspired investigation. While I gave my best efforts to understand and apply the principles of a realist research, I was limited by my inexperience and capability as a realist researcher. Having gained the experience of undertaking a realist research, on reflection, I may have used more focused follow-up questions during the interviews, workshops and the online survey to drill further into revealing the realist mechanisms that influence the successful implementation of LST initiatives.

\subsection{Limitations}

Two interactive workshops with a limited number of senior operational and clinical leaders (nine and 10 respectively) from the New Zealand health system were used to test and refine the key elements and construct the maturity matrix. Given the seniority and work commitments of senior leaders, it is unusual for researchers to have participants of this calibre and their employers to contribute two days to doctoral research. Participants who attended were keen to share the knowledge and experience and contribute to the international literature. In the introduction session, participants shared their reasons for attending the workshops.

I've decided at my time of life l'll only do things that I find interesting; this is really interesting, but actually really important as well. I think the whole way the system works, and process is incredibly important to outcomes and certainly with the population I see on a daily basis (senior primary care clinician).

I'm here because you guys put yourself on the line and made it work, and that's important. But also, I do a lot of work overseas and one of the things that keeps on coming back at us is fantastic stuff happens in New Zealand and we don't write it up enough and we don't publish it enough. Again, power to you for actually doing 
the work that enables the learnings from this country to be used in other health constituencies (senior DHB leader).

Group consensus on the key elements was reached quite quickly which led me to contemplate if there were enough participants present or if there was a lack of variation in the participants, that is, did I select like-minded people? A wider group of participants may have had other views.

However, listening to the audio recorded discussion later in the evening of the first workshop emphasised that early consensus was reached because all participants firmly believed that a maturity matrix with key elements that support successful implementation of LST initiatives would be a practical way to support the notion of a learning system in the New Zealand health system. Participants also discussed that output of this research would be the first step and that the use of the maturity matrix would form an iterative improvement process that will build on this research. They were practical about not looking for a perfect solution but rather using this research to make a start on formulating the programme architecture underpins efforts to successfully implement LST initiatives that system leaders can use. Nonetheless, a wider group of participants, particularly more Māori and Pacific leaders, may have generated more robust discussion on the key elements, the content of the maturity matrix, and identifying the contexts and the mechanisms.

Another limitation of the study was the testing of the maturity matrix in that it was tested with only three Alliance leadership teams. More testing is required to confirm the fidelity and structural validity of the maturity matrix. Peer assessment at regional level or with similar size districts will promote peer learning, provide a check-and-balance approach to the learning and strengthen the consistency in the use of the maturity matrix. Sharing of results among Alliances will contribute to regional or national learning communities and enhance the notion of a learning system.

This research required significant qualitative analysis of data. To ensure qualitative rigour, I used NVivo computer software for initial thematic analysis, referred to transcripts to cross-check contexts, mechanisms and outcomes, and sought advice from my supervisors. However, having a second reviewer to scrutinise the qualitative coding would have added rigour to qualitative analysis.

The findings of this research do not provide a comprehensive view of elements and contextual factors that influence successful implementation of LST initiatives. While the research triangulated evidence from several sources, literature is still young, and evidence is constantly 
emerging. In line with one of the five ideas of realist research, the findings provide a better understanding for system leaders on the programme architecture that underpins efforts to successfully implement LST initiatives in the New Zealand health system, and not the final truth. Additionally, in accordance with the other four ideas of realist research, given the emergent and generative nature of complex adaptive systems, the contextual factors described in this research will continue to evolve triggering new mechanisms that will influence interactions and behaviours of those involved in implementation of LST initiatives. 


\subsection{CONCLUDING CHAPTER}

\subsection{Future of the SLM programme}

The original intervention logic of the SLM programme (Figure 3.2) identified several external factors that would affect the short, medium- and long-term impacts of the programme. The research findings confirmed these factors as contextual factors that influence the successful implementation of LST initiatives, such as the SLM programme. Additionally, the intervention logic correctly assumed that success of the programme relied on the intrinsic motivation of the health providers to improve health outcomes; districts having a functional Alliance; high-trust relationships among Alliance members; and the willingness of funders to change models of care and funding arrangements. The intervention logic identified several short-, medium- and longterm impacts of the SLM programme. While the intervention logic for the SLM programme was in line with the international evidence for successful implementation of LST initiatives, the programme was quite ambitious in the outcomes and influence it was seeking. There was an underlying assumption that Alliances will step up and improve their capacity and capability for implementing the programme through a continuous learning process. The implementation, however, identified the need for key elements that support capacity and capability for large-scale change and the influence of contextual factors in Alliances' ability to successfully implement the SLM programme and sustain their efforts.

This research aimed to identify the key elements that support successful implementation of LST initiatives, describe what these elements mean for the New Zealand health system so New Zealand Alliances could consciously and deliberately increase their understanding of the key elements, and the contexts and mechanisms that influence New Zealand Alliances' (or future locality networks') ability to successfully implement LST initiatives. Figure 15.1, adapted from Middleton, et al. [225], show an updated intervention logic for the SLM programme with a summary of key elements; mechanisms triggered by local and national contexts identified in this research; and the short- and medium-term outcomes of the programme.

This research confirmed that sustained high-trust relationships triggered by a distributed leadership style from DHBs, supported by key enablers, are the pre-conditions needed for a mature Alliance, which provides a critical platform for successful implementation of LST initiatives. Those DHBs who understand and improve the pre-conditions will continue to successfully implement the SLM programme and those who do not will continue to struggle and 
or view it as a compliance exercise. Without dedicated resources and support from the $\mathrm{MoH}$ and DHBs to improve the pre-conditions, the SLM programme is unlikely to be a sustainable policy.

\subsection{Research contribution}

This research is first to describe in detail the development and the implementation of the SLM programme. The programme is unique in its collaborative design with a continuous improvement approach and implementation through Alliances and has generated interest of overseas health system leaders. The rich description of the programme and the insights and experience from implementation offered in this research make a significant contribution to health system transformation literature in New Zealand and internationally.

Although key elements that support successful implementation of LST initiatives exist in international literature, this research is a first to identify key elements that need to be present to increase the chances of success for implementation of LST initiatives in the New Zealand health system. It takes into consideration the New Zealand context, in particular Te Tiriti o Waitangi.

Further, this research is ground-breaking in the construction of a maturity matrix for the New Zealand health system leaders and trust-based networks such as Alliances to use to assess and enhance their capacity and capability for successfully implementing LST initiatives. Workshop participants, who were senior health system leaders, considered the SCIROCCO self-assessment tool available for European regions to assess their readiness to deliver integrated care. However, they did not consider this tool to be suitable in the New Zealand context.

The CMO theories that influence successful implementation of LST initiatives reported in this research add to the health system transformation literature, which is still considered young and scarce. 


\begin{tabular}{|c|c|c|c|c|c|c|c|c|}
\hline INPUTS & Key elements & ACTIVITIES & Key elements & OUTPUTS & Key elements & $\begin{array}{l}\text { (Short) } \\
\text { (ShICOIES }\end{array}$ & Key elements & (Medium) \\
\hline $\begin{array}{l}\text { Alliance } \\
\text { structure - } \\
\text { ALTs and } \\
\text { SLATs, agreed } \\
\text { charter, } \\
\text { shared } \\
\text { decision- } \\
\text { making } \\
\text { framework, } \\
\text { supported by } \\
\text { DHB } \\
\text { Data and } \\
\text { insights } \\
\text { Quality } \\
\text { improvement } \\
\text { tools } \\
\text { Shared vision } \\
\text { and goals for } \\
\text { the district }\end{array}$ & $\begin{array}{l}\text { An alliancing way of } \\
\text { working } \\
\text { Dedicated resources } \\
\text { and time } \\
\text { Integrated health } \\
\text { information \& } \\
\text { analytic capability } \\
\text { Reasoning } \\
\text { High-trust } \\
\text { relationships are } \\
\text { sustained over time } \\
\text { and alliance } \\
\text { members can foster a } \\
\text { collective approach } \\
\text { to implementation of } \\
\text { a programme that is } \\
\text { co-designed with } \\
\text { sector }\end{array}$ & $\begin{array}{l}\text { Establish } \\
\text { project teams } \\
\text { for each SLM } \\
\text { Review } \\
\text { evidence to } \\
\text { determine } \\
\text { improvement } \\
\text { focus } \\
\text { Agree } \\
\text { improvement } \\
\text { actions } \\
\text { Select } \\
\text { contributory } \\
\text { measures } \\
\text { Implement the } \\
\text { SLM plan } \\
\text { Monitor, } \\
\text { report, learn, } \\
\text { and adapt }\end{array}$ & $\begin{array}{l}\text { Commitment to Te Tiriti } \\
\text { Involved whanau \& } \\
\text { communities } \\
\text { Clinical leadership and } \\
\text { involvement } \\
\text { Intelligent } \\
\text { commissioning } \\
\text { Understanding of equity } \\
\text { Continuous } \\
\text { improvement } \\
\text { Reasoning } \\
\text { Involvement of frontline } \\
\text { staff, whanau and } \\
\text { communities in deciding } \\
\text { improvement actions } \\
\text { with system leaders } \\
\text { fostering collective } \\
\text { leadership, celebrating } \\
\text { innovations and having } \\
\text { confidence in staff to } \\
\text { work differently } \\
\text { Capacity and capability } \\
\text { System leadership } \\
\text { 'New power' approach } \\
\text { System accountability } \\
\text { environment }\end{array}$ & $\begin{array}{l}\text { MoH } \\
\text { approved SLM } \\
\text { plan } \\
\text { Project plans } \\
\text { for } \\
\text { improvement } \\
\text { actions } \\
\text { Quarterly } \\
\text { reporting to } \\
\text { alliance and } \\
\text { MoH } \\
\text { Subsequent } \\
\text { plans reflect } \\
\text { learning from } \\
\text { successes and } \\
\text { failures of } \\
\text { past plans and } \\
\text { processes }\end{array}$ & $\begin{array}{l}\text { Reasoning } \\
\text { High-trust } \\
\text { relationships are } \\
\text { sustained over } \\
\text { time and alliance } \\
\text { members can } \\
\text { navigate } \\
\text { through } \\
\text { emerging } \\
\text { challenges and } \\
\text { staff prioritise } \\
\text { their work to } \\
\text { stay focused on } \\
\text { the shared vision }\end{array}$ & $\begin{array}{l}\text { Increase } \\
\text { maturity of } \\
\text { alliances } \\
\text { Use of constant } \\
\text { feedback loops } \\
\text { for continuous } \\
\text { improvement } \\
\text { Commissioning } \\
\text { and investment } \\
\text { driven by } \\
\text { evidence } \\
\text { Sharing of good } \\
\text { practices } \\
\text { Improved } \\
\text { analytic } \\
\text { capability } \\
\text { Achievement of } \\
\text { SLM milestones }\end{array}$ & $\begin{array}{l}\text { Commitment to Te } \\
\text { Tiriti } \\
\text { Involved whanau \& } \\
\text { communities } \\
\text { Clinical leadership and } \\
\text { involvement } \\
\text { Understanding of } \\
\text { equity } \\
\text { Continuous } \\
\text { improvement } \\
\text { Reasoning } \\
\text { Staff feel supported } \\
\text { to try new initiatives, } \\
\text { involve whānau, iwi } \\
\text { and communities in } \\
\text { the programme } \\
\text { implementation, and } \\
\text { system leaders focus } \\
\text { their efforts beyond } \\
\text { the accountability } \\
\text { measures and their } \\
\text { unit or organisation } \\
\text { Continuous } \\
\text { improvement culture } \\
\text { System accountability } \\
\text { environment }\end{array}$ & $\begin{array}{l}\text { Successful } \\
\text { implementation } \\
\text { of the SLM plan } \\
\text { Sustained high- } \\
\text { trust } \\
\text { relationships } \\
\text { across system } \\
\text { Stronger } \\
\text { partnership with } \\
\text { whānau, iwi and } \\
\text { communities } \\
\text { Continuous } \\
\text { system } \\
\text { improvement } \\
\text { approach } \\
\text { becomes norm } \\
\text { Reduced health } \\
\text { inequities for } \\
\text { Māori and } \\
\text { Pasifika }\end{array}$ \\
\hline
\end{tabular}

Figure 15.1: Updated SLM programme intervention logic with summary of realist mechanisms triggered by local and national contexts, adapted from Middleton, et al. [225] 


\subsection{Future research}

This research was the first in New Zealand to use a realist logic of enquiry nested within the macro framing of complex adaptive systems. Use of a realist approach is becoming common in the health research community and this research adds to the realist methods literature. There are opportunities for future research to further test and refine the programme architecture underpins efforts to successfully implement LST initiatives. These include:

- Using insights from other LST initiatives to refine the key elements that need to be present to increase chances of success with these initiatives.

- Further testing and refining of the maturity matrix to confirm its structural validity and building a peer assessment component so networks such as Alliances can compare or benchmark against each other. There is an opportunity to create a digital platform for the maturity matrix so networks such as Alliances could electronically record, monitor and share their assessments.

- This research revealed the critical role of networks such as Alliances in the successful implementation of LST initiatives. Literature on these networks is still emerging and there is an opportunity to undertake further research on the inner workings of these networks and their role in implementing LST initiatives.

\subsection{Research dissemination and uptake of recommendations}

This research offers insights that could be relevant to the New Zealand health system and health systems world-wide.

The New Zealand health system is at a pivotal moment with the release of the Health and Disability System Review (HDSR). The health system response to COVID-19 highlighted to the government the fragmented nature of the system and identified opportunities to strengthen the system. The newly elected government plans to reform the health system drawing on the recommendations of the HDSR and lessons from the COVID-19 response [226]. The reform has begun with the establishment of a transition team in the Department of Prime Minister and Cabinet (DPMC). If the recommendations of the HDSR are implemented, Alliances will be replaced with locality networks.

I have shared the maturity matrix with several Alliance leadership teams so they can use to assess their capacity and capability to implement the SLM programme and to assess their readiness for the locality networks. 
I plan to share findings of this research using tools and opportunities available to me. This include:

- Sharing an executive summary of my thesis (3-4 pages long) along with the final full thesis with participants who requested this through their consent forms. This includes New Zealand and overseas participants and participants from non-health sector.

- Disseminating the executive summary and the maturity matrix with various sector groups in New Zealand such as, the DHB and PHO CEOs and clinical leaders, and the Alliance leadership teams.

- Sharing the maturity matrix as part of the guidance materials for the ongoing implementation of the SLM programme and to use the research findings to evolve the programme so it remains relevant in the future New Zealand health system landscape.

- Sharing the executive summary and maturity matrix with the Health Quality and Safety Commission for them to use as part of their national quality improvement programmes.

- Sharing the executive summary, the maturity matrix and the full final thesis to the Chief Executive of the transition team at the DPMC leading the New Zealand health system reform so they may use the maturity matrix to prototype locality networks.

- Using social media platforms, such as Linkedln, to share snippets of key findings.

Further, I plan to share key findings and recommendations with the wider research community nationally and internationally through peer-reviewed publications, presentations at conferences and webinars.

Finally, health system transformation is an area I am passionate about and see myself continuing to progress my professional career in. This research gives me an important foundation in my future endeavours as a health system leader.

\subsection{Concluding remarks}

This research builds on health system transformation literature and emphasises the use of LST initiatives to make the desired shift most health systems are seeking to deliver health care that is people-centred, holistic, and one that addresses health inequities. The 1990s organisational reforms in New Zealand and the rise of 'new public management' contributed to a transactional paradigm in which delivery of health care is fragmented and focused on institutional arrangements and health care professionals. Over last two decades, recognition of health systems as complex adaptive systems has challenged the transactional paradigm and LST 
initiatives that capitalise on key features of complex adaptive systems are theorised to achieve the desired shift.

LST initiatives span across geographic, organisational and professional boundaries and challenge current ways of thinking, processes and behaviour patterns. Successful implementation of LST initiatives requires systems mind set in which power is shared among system leaders and the focus is on interactions and behaviours of system agents while taking into consideration the social contexts in which the system is operating. These initiatives require integrated changes in structures, processes and behaviour of system actors.

Senior leaders of the system, especially from organisations with largest amount of resources, have to let go of control and the traditional 'old power' approach and embrace 'new power' values that comes from relationships and connections, and uses people's intrinsic motivation to influence and call for action to achieve a shared purpose or vision.

This research bridges the gap between the theory of LST initiatives and the reality of implementing these initiatives in a system where change efforts are constantly filtered and adapted by frontline health care staff and contextual factors in which the change is implemented heavily influences its success. The research affirms the use of informal trust-based networks, such as Alliances, to bring many health system agents together to deliver integrated people-centred health care. Performance of these networks is underpinned by unprecedented levels of trust, mutual understanding, informal rules, and commitment among partners to work towards a shared vision and overcome great challenges in a complex system that no single player is likely to achieve individually.

The research findings provide a rich description of the programme architecture that underpins efforts to successfully implement LST initiatives in the New Zealand context. The programme architecture includes the maturity matrix for a set of 10 key elements that increases the chances of success with implementation of LST initiatives and CMO theories that enhance understanding of mechanisms that influence behaviours of system actors, which then influences outcomes of programmes or interventions.

This research found that understanding of this programme architecture by health system leaders is critical. System leaders create the right conditions for collective responsibility of LST initiatives by embracing the 'new power' approach. 
My findings suggest that the maturity matrix constructed in the research provides a practical tool for health system leaders to use to consciously and deliberately improve their understanding of this programme architecture. A constant use of the maturity matrix will create feedback loops that will support a continuous improvement approach. A continuous improvement approach supports the notion of a learning system. A learning system nudges the behaviour of the system out of equilibrium and builds momentum for change.

In conclusion, successful implementation of LST initiatives requires health system agents to muddle through many complex and unexpected factors and nurture trust and relationships among all system actors including system leaders, middle managers, health care professionals, and most importantly people, whanau, and communities. Next steps in this research are to test and refine the maturity matrix with the newly proposed locality networks and implementation of other LST initiatives in New Zealand and overseas. 


\section{PART E: APPENDICES}

\section{AND REFERENCES}

This last part of the thesis contains documents that support the main body of the thesis. Part $\mathrm{E}$ includes two sub-parts: appendices; and references.

The appendices include participant information sheets, consent forms, workshop schedules, and details on how the workshops were conducted.

The references include all the sources of literature used in the writing of the thesis. 
Appendix 1: Structure of the New Zealand health system [66]

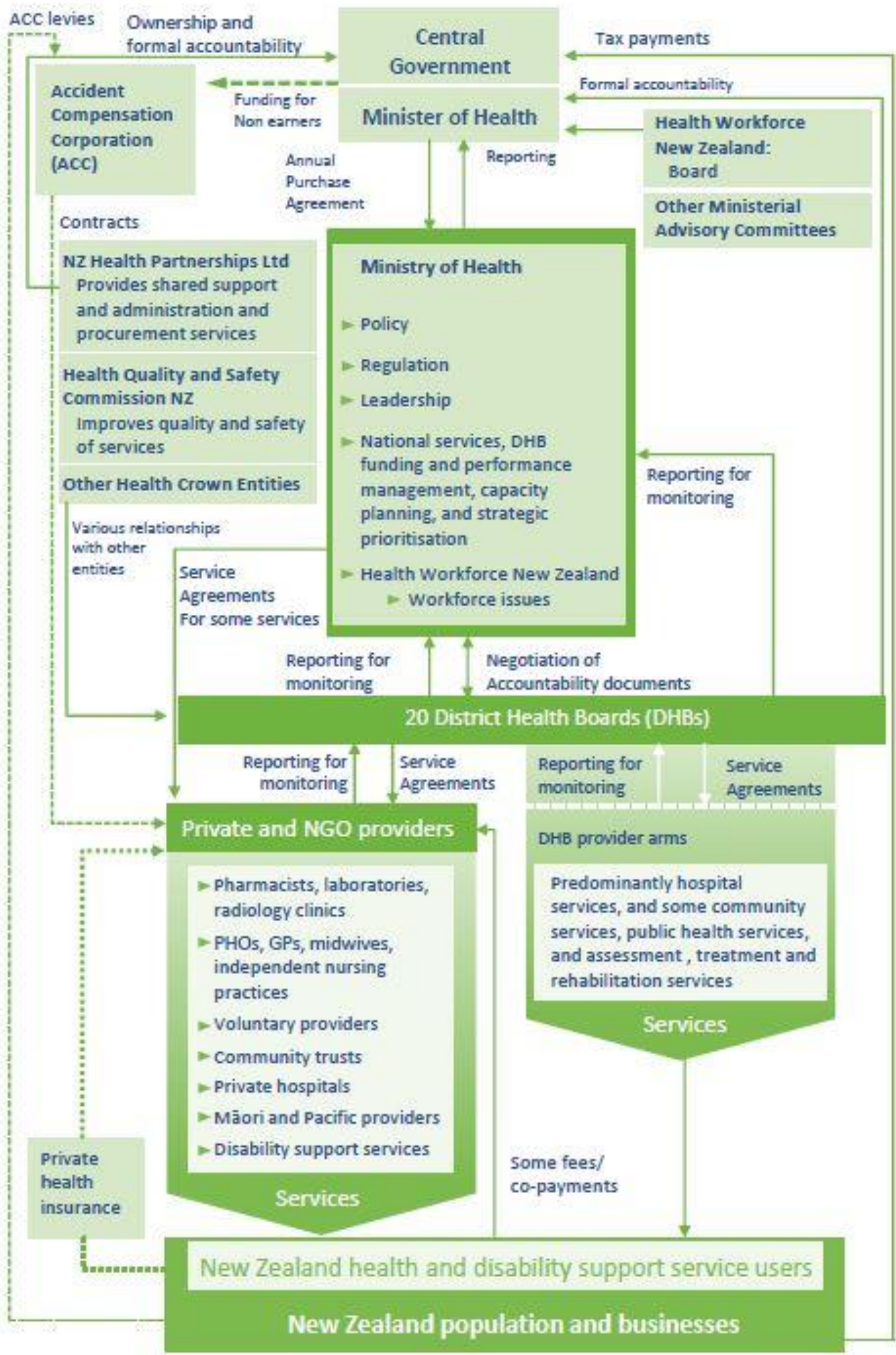




\begin{tabular}{|c|c|c|}
\hline Study & Aims and Setting & Key elements for LST initiatives \\
\hline $\begin{array}{l}\text { Baker and Axler } \\
\text { [122] }\end{array}$ & $\begin{array}{l}\text { Creating a high performing } \\
\text { health care system for } \\
\text { Ontario - a review of } \\
\text { evidence of high } \\
\text { performing health care } \\
\text { systems, their } \\
\text { characteristics, key drivers } \\
\text { and what Ontario can learn } \\
\text { from them. }\end{array}$ & 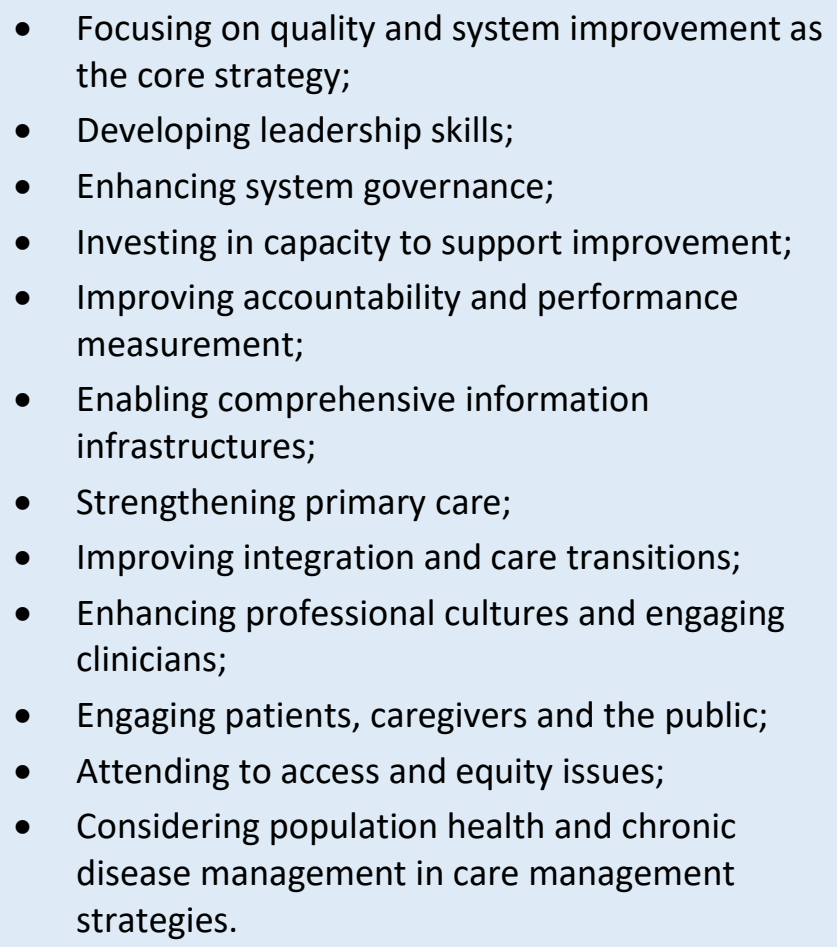 \\
\hline Berta, et al. [199] & $\begin{array}{l}\text { Pursuing insights into } \\
\text { system-level diffusion and } \\
\text { whole systems change in } \\
\text { evidence-informed nursing } \\
\text { service delivery innovations } \\
\text { in Canada }\end{array}$ & $\begin{array}{l}\text { System readiness } \\
\text { - } \quad \text { Emerging need for change } \\
\text { - } \quad \text { Adaptable micro-systems } \\
\text { - } \quad \text { Mechanisms that support change } \\
\text { System capacity } \\
\text { - Champions } \\
\text { - } \quad \text { resources } \\
\text { System alignment } \\
\text { - Common goals, beliefs and values among } \\
\text { - stakeholders } \\
\text { synergies }\end{array}$ \\
\hline Best, et al. [1] & $\begin{array}{l}\text { Description of a realist } \\
\text { review approach to an LST } \\
\text { initiative using a system } \\
\text { transformation project in } \\
\text { Saskatchewan Canada. } \\
\text { To provide wider lessons to } \\
\text { implement and support LST } \\
\text { initiatives and for realist } \\
\text { reviewers. }\end{array}$ & $\begin{array}{l}\text { - } \quad \text { Blend designated and distributed leadership; } \\
\text { - Establish feedback loops; } \\
\text { - Attend to history; } \\
\text { - Engage physicians; } \\
\text { - Include patients and families }\end{array}$ \\
\hline Evans, et al. [3] & $\begin{array}{l}\text { Conceptual framework for } \\
\text { successful and sustainable } \\
\text { implementation of } \\
\text { integrated care } \\
\text { interventions in Ontario } \\
\text { Canada }\end{array}$ & $\begin{array}{l}\text { Three most capabilities that shape organisation } \\
\text { capacity: } \\
\text { - Leadership approach } \\
\text { - Clinician engagement and leadership } \\
\text { - Readiness for change }\end{array}$ \\
\hline
\end{tabular}




\begin{tabular}{|c|c|c|}
\hline Study & Aims and Setting & Key elements for LST initiatives \\
\hline & & $\begin{array}{l}\text { Other capabilities identified, group in three } \\
\text { concepts, were: } \\
\text { Basic structures } \\
\text { - } \quad \text { Physical features } \\
\text { - } \text { Resources } \\
\text { - } \quad \text { Accountability } \\
\text { - Information technology } \\
\text { - } \quad \text { Organisational/Network design } \\
\text { People and values } \\
\text { - } \quad \text { Leadership approach } \\
\text { - Clinical engagement and leadership } \\
\text { - } \quad \text { Organisational/Network culture } \\
\text { - } \quad \text { Focus on patient-centeredness and engagement } \\
\text { - Commitment to learning } \\
\text { - Work environment } \\
\text { - } \quad \text { Readiness for change } \\
\text { Key processes } \\
\text { - Partnering } \\
\text { - } \quad \text { Delivering care } \\
\text { - } \text { Impeasuring performance }\end{array}$ \\
\hline $\begin{array}{l}\text { Matheson, et al. } \\
\text { [141] }\end{array}$ & $\begin{array}{l}\text { Evaluation of Healthy } \\
\text { Families New Zealand - a } \\
\text { systems change } \\
\text { intervention to prevent } \\
\text { chronic diseases in } 10 \\
\text { communities }\end{array}$ & $\begin{array}{l}\text { Factors that facilitate the quality of implementation } \\
\text { of the initiative: } \\
\text { - Workforce } \\
\text { - Leadership } \\
\text { - Relationships and networks } \\
\text { - } \quad \text { Resources } \\
\text { - Knowledge and data }\end{array}$ \\
\hline $\begin{array}{l}\text { McGrath, et al. } \\
\text { [190] }\end{array}$ & $\begin{array}{l}\text { Implementing and } \\
\text { sustaining transformational } \\
\text { change in health care in } \\
\text { South Australia - NSW } \\
\text { Health and Flinders Medical } \\
\text { Centre }\end{array}$ & $\begin{array}{l}\text { - Leadership by senior executives, } \\
\text { - Clinical leadership, } \\
\text { - Team-based problem solving, } \\
\text { - A focus on the patient journey, } \\
\text { - Access to data, } \\
\text { - Ambitious targets, } \\
\text { - Strong performance management, } \\
\text { - A process for maintaining improvement }\end{array}$ \\
\hline Perla, et al. [30] & $\begin{array}{l}\text { Literature scan relating to } \\
\text { current thinking, practice } \\
\text { and knowledge that inform } \\
\text { large-system improvement } \\
\text { initiatives in health care. }\end{array}$ & $\begin{array}{l}\text { Planning and infrastructure } \\
\text { - Vision and aim } \\
\text { - Intervention } \\
\text { - Management } \\
\text { - Resources }\end{array}$ \\
\hline
\end{tabular}




\begin{tabular}{|c|c|c|}
\hline Study & Aims and Setting & Key elements for LST initiatives \\
\hline & & $\begin{array}{l}\text { Individual, Group, Organisational and System factors } \\
\text { - } \quad \text { Champions/change agents } \\
\text { - Leadership roles } \\
\text { - } \quad \text { Capability and capacity development } \\
\text { - } \quad \text { Learning networks } \\
\text { - } \quad \text { Social networks } \\
\text { - Organisational and system capability } \\
\text { - Organisational and system culture } \\
\text { The process of change } \\
\text { - Change theory used } \\
\text { Performance measures and evaluation } \\
\text { - Data infrastructure } \\
\text { - Measurement and feedback systems }\end{array}$ \\
\hline $\begin{array}{l}\text { Taylor, et al. } \\
\text { [200] }\end{array}$ & $\begin{array}{l}\text { To identify the factors } \\
\text { associated with high } \\
\text { performing hospitals and } \\
\text { practical strategies for } \\
\text { improvement. }\end{array}$ & $\begin{array}{l}\text { Positive organisational culture } \\
\text { - } \quad \text { Respect between colleagues, disciplines and } \\
\text { - } \quad \text { departments } \\
\text { - } \quad \text { No blame culture } \\
\text { - } \quad \text { Recognition and reward } \\
\text { Senior management support } \\
\text { - } \quad \text { Support and resources } \\
\text { - } \quad \text { Access and visibility } \\
\text { - } \quad \text { Commitment } \\
\text { Effective performance monitoring } \\
\text { - } \quad \text { Measurement framework } \\
\text { - } \quad \text { IT infrastructures } \\
\text { - } \text { Accountability } \\
\text { - Using data for CQI } \\
\text { Building and maintaining a proficient workforce } \\
\text { - Hiring and retaining good staff } \\
\text { - } \text { Aligning staff values and behaviour to } \\
\text { - } \text { organisational vision (and not professional } \\
\text { - } \text { agenda) } \\
\text { - } \text { Effective dissemination of policy and processes } \\
\text { - Mutual respect } \\
\text { - }\end{array}$ \\
\hline
\end{tabular}




\begin{tabular}{|c|c|c|}
\hline Study & Aims and Setting & Key elements for LST initiatives \\
\hline & & $\begin{array}{l}\text { Expertise-driven practice } \\
\text { - Clinical leadership and engagement } \\
\text { - Trust and empowerment for innovation } \\
\text { Interdisciplinary teamwork } \\
\text { - Teams and professions working together to } \\
\text { - } \quad \text { achieve common goals } \\
\text { - collance, horizontal team that involves } \\
\text { - Collaboration with primary care and social } \\
\text { agencies }\end{array}$ \\
\hline $\begin{array}{l}\text { Urtaran- } \\
\text { Laresgoiti, et al. } \\
\text { [37] }\end{array}$ & $\begin{array}{l}\text { Realist evaluation of } \\
\text { system-wide } \\
\text { transformation in the } \\
\text { Basque Country and to } \\
\text { provide transferable } \\
\text { lessons for decision makers }\end{array}$ & $\begin{array}{l}\text { - Leadership, } \\
\text { - Commitment, } \\
\text { - } \text { betlaborative decision-making and relationships } \\
\text { - Vision and narrative } \\
\text { - Developing new staff roles, } \\
\text { - Investing in integrated electronic health records, } \\
\text { - Stratifying the population by risk, } \\
\text { - Facilitating bottom-up innovation }\end{array}$ \\
\hline Willis, et al. [198] & $\begin{array}{l}\text { Realist rapid review of } \\
\text { literature to examine how } \\
\text { interventions are } \\
\text { influenced by contexts to } \\
\text { influence sustainability of } \\
\text { cultural change in health } \\
\text { systems }\end{array}$ & $\begin{array}{l}\text { Six guiding principles identified: } \\
\text { - } \quad \text { Align vision and action } \\
\text { - Make incremental changes within a } \\
\text { - } \text { comprehensive transformation strategy } \\
\text { - Promote staftributed leadership } \\
\text { - Create collaborative relationships } \\
\text { - Continuously assess and learn from change }\end{array}$ \\
\hline
\end{tabular}




\section{Framework for building capacity and capability for improvement in the New Zealand health system: a realist approach}

\section{INFORMATION SHEET FOR PARTICIPANTS}

You are invited to take part in this research. Please read this information before deciding whether or not to take part. If you decide to participate, thank you. If you decide not to participate, thank you for considering this request.

\section{What is the aim of the project?}

This research is about building capacity and capability for improvement in the New Zealand health system and thereby, shifting towards a learning system. The research is nested within the framing of complex adaptive systems. Small-scale change efforts at a micro level are insufficient to improve the performance and outcomes of the health system. LST initiatives that capitalise on the key elements of a complex adaptive system, such as the use of informal networks and distributed leadership, together with a deep understanding of the contexts that influence successful implementation of LST initiatives, are more likely to bring the different component parts of the system to work together successfully and sustainably.

By LST initiatives I am referring to co-ordinated, system-wide change involving multiple organisations that takes into consideration the relationships, interactions and behaviours of those in the system. The goal of LST initiatives is to stimulate communication and learning among different component parts of the system and for those in the system to understand the features of a complex adaptive system; thereby building the capacity and capability for improvement and shifting towards a learning system.

One such LST initiative is the System Level Measures (SLM) programme, which was developed with the sector leaders and clinicians. Alliances made up of District Health Board of domicile with their primary, hospital and community partners, are responsible for leading the implementation of the SLM programme.

This research aims to use the insights gained from the implementation of the SLM programme; first, to identify key elements that enable LST initiatives in the New Zealand health system; second, to identify how local context influence the key elements; and finally, to stimulate 
communication and learning within Alliances through the development and testing of a maturity matrix for the key elements.

\section{Who am I?}

My name is Kanchan Sharma and I am a Doctoral student in Faculty of Health at Victoria University of Wellington. I am also an employee of the $\mathrm{MoH}$, leading the SLM programme.

\section{Conflict of interest}

The inside knowledge and the first-hand experience with leading the implementation of the SLM programme gives me invaluable insights into the key elements that enable large system transformations in New Zealand and how the local contexts influence these elements.

However, my dual role as a Ministry employee and a researcher presents a conflict of interest that can be both real and perceived. It is therefore important for me to distinguish between the privileges I have from my position in respect to the research population and the genuine ethical issues that might arise, such as an impact on voluntary participation and the ability for participants to be honest in their feedback.

Participation in the research is voluntary. You do not have to participate if you do not want to and you do not have to give me a reason for your decision.

Data collected during this research will only be used for my thesis. I will not use the data collected for my Ministry role or disclose the data to any employee of the $\mathrm{MH}$.

The $\mathrm{MoH}$ may be interested in the information collected during this research or may in future use the output of the research, in particular the maturity matrix, in conjunction with the SLM programme. Any information shared with the $\mathrm{MoH}$ will be in accordance with the research confidentiality agreements. This means that data from the research will be combined and all identification information removed. 
Workshop participants

The workshops will be facilitated by $\operatorname{Dr} X X X$, who is the SLM programme clinical lead and a PHO employee. This may also have a real and perceived conflict of interest.

Dr XXX has signed a confidentiality agreement to keep the identity of participants and the information obtained from the workshops confidential. XXX will not discuss the information obtained with anyone except with my supervisors, me, and potentially with the participants involved in the workshops.

\section{Alliance leadership team participants}

The Alliance meetings will be facilitated by $\operatorname{Dr} X X X$, who is the SLM programme clinical lead and a PHO employee. This may also have a real and perceived conflict of interest.

$\operatorname{Dr} X X X$ has signed a confidentiality agreement to keep the identity of participants and the information obtained from the meeting with Alliances confidential. XXX will not discuss the information obtained with anyone except with my supervisors, me, and potentially with the participants involved in the meeting.

This research is using the insights from the SLM programme but is not undertaking a formal evaluation of the programme. If participants want to discuss or provide any feedback about the SLM programme, they should email Ministry directly (SLM@health.govt.nz).

This research has been approved by the Victoria University of Wellington Human Ethics Committee (application reference number 0000027356).

If I experience or am challenged by any conflicts of interest as the research proceeds, I will seek advice and support from my supervisors and the University Human Ethics Committee to appropriately manage it. 


\section{How can you help?}

Interview participants

If you agree to take part, I will interview you at your place of work, or using phone or skype technology between July and November 2019 (date, time and venue to be confirmed). The interview will take between 45-60 minutes. I will audio record the interview with your permission and write it up later.

You can choose to not answer any question or stop the interview at any time, without giving a reason. You can withdraw from the study by contacting me at any time before 30 November 2019. If you withdraw, the information you provided will be destroyed or returned to you.

Workshop participants

You have been invited to participate because of your expertise in the health system or a complex system, its successes and challenges; and your understanding of system improvement.

If you agree to take part, you will be part of two workshops that will be held in Wellington on 21 and 28 August 2019.

The workshops will be facilitated by $\operatorname{Dr} X X X . X X X$ will ask you and other participants questions about the key elements necessary to enable LST initiatives in the New Zealand health system; description and maturity matrix of the key elements; and local contexts, behaviours and processes that influence the local district health systems' ability to implement LST initiatives successfully and sustainably.

The workshops will take approximately six hours each. I will audio record the workshops with your permission and write it up later. You can request for the recording to stop at any time during the workshop to enable an 'off the record' conversation.

The information shared during the workshops is confidential. That means after the workshop, you may share your own experience but not reveal the identities or experiences of others. Doing so may jeopardise the anonymity of participants and their employing organisations.

You can withdraw from the workshop at any time before the workshop begins. You can also withdraw while the workshop is in progress. However, it will not be possible to withdraw the information you have provided up to that point as it will be part of discussion with other participants. 
Survey participants

You have been invited to participate because of your potential involvement in the implementation of the SLM programme. If you agree to take part, you will complete an online survey. The survey will ask you questions about your experience on the implementation of the SLM programme. The survey will take you about 10 minutes to complete.

Alliance leadership team participants

You have been invited to participate, as part of an Alliance leadership team, to test the maturity matrix developed to enhance the Alliances' capacity and capability for improvement.

If you agree to take part, you will be present at the Alliance leadership team meeting and participate in the testing process.

$\operatorname{Dr} X X X$, the SLM programme clinical lead, will facilitate the meeting. XXX will ask the Alliance leadership team to assess their Alliance on the maturity matrix for the key elements necessary to enable implementation of LST initiatives successfully and sustainably.

The purpose of the testing process is not to judge the maturity or functionality of the Alliance. It is to test if the maturity matrix is functioning as a learning tool and stimulating conversations at the meeting, and how simple and practical it is to use. This will help me refine the maturity matrix. The Alliance can keep the tool and any insights they gain to use for their improvement purposes. The maturity matrix will eventually be available for all the Alliances.

The assessment process will take approximately one hour. I will audio record the meeting with your permission and write it up later. You can request for the recording to stop at any time during the meeting to enable an 'off the record' conversation.

\section{What will happen to the information you give?}

This research is confidential. This means that the researcher named below will be aware of your identity, but the research data will be combined, and your identity will not be revealed in any reports, presentations, or public documentation.

Some of the information provided by you may be used as direct quotes in the reports. However, all identification information will be removed. 
Interview participants

Only my supervisors, the transcriber (who will be required to sign a confidentiality agreement), and I will read the notes or transcript of the interview. The interview transcripts, summaries and any recordings will be kept securely and destroyed on 31 October 2022.

\section{Workshop participants}

Only my supervisors, the workshop facilitator and the transcriber (both of whom will be required to sign a confidentiality agreement), and I will read the notes or transcript of the workshop. The workshop transcripts, summaries and any recordings will be kept securely and destroyed on 31 October 2022.

\section{Survey participants}

This survey is anonymous. This means that nobody, including the researcher will be aware of your identity. By answering it, you are giving consent for me to use your responses in this research. Your answers will remain completely anonymous and unidentifiable. Once you submit the survey, it will be impossible to retract your answer. Please do not include any personal identifiable information in your responses.

\section{Alliance leadership team participants}

Only my supervisors, the meeting facilitator and the transcriber (both of whom have signed confidentiality agreements), and I will read the notes or transcript of the meeting. The meeting transcripts, summaries and any recordings will be kept securely and destroyed on 31 October 2022.

\section{What will the project produce?}

The information from my research will be used in my PhD thesis, academic publications and presentation to conferences and Alliance teams or equivalent in New Zealand and overseas.

\section{If you accept this invitation, what are your rights as a research participant?}

You do not have to accept this invitation if you don't want to. If you do decide to participate, you have the right to:

- choose not to answer any question 
- $\quad$ ask for the recorder to be turned off at any time during the interview

- $\quad$ withdraw from the study before 30 November 2019

- $\quad$ ask any questions about the study at any time

- receive a copy of your interview transcript

- be able to read any reports of this research by requesting a copy through the consent form. If you have any questions or problems, who can you contact?

If you have any questions, either now or in the future, please feel free to contact me:

Student:

Name: Kanchan Sharma

University email address:

Kanchan.sharma@vuw.ac.nz
Supervisor:

Name: Professor Jackie Cumming

Role: Director, Health Services Research Centre

School: Faculty of Health

Phone: 044636567

Jackie.cumming@vuw.ac.nz

\section{Human Ethics Committee information}

If you have any concerns about the ethical conduct of the research, you may contact the Victoria University HEC Convenor: Dr Judith Loveridge. Email hec@vuw.ac.nz or telephone +64-4-463 6028. 


\section{Framework for building capacity and capability for improvement in the New Zealand health system: a realist approach \\ CONSENT TO INTERVIEW}

This consent form will be held for three years.

Researcher: Kanchan Sharma, Faculty of Health, Victoria University of Wellington.

- I have read the Information Sheet and the project has been explained to me. My questions have been answered to my satisfaction. I understand that I can ask further questions at any time.

- I agree to take part in an audio recorded interview.

I understand that:

- I may withdraw from this study at any point before 30 November 2019 , and any information that I have provided will be returned to me or destroyed.

- $\quad$ The identifiable information I have provided will be destroyed on 31 October 2022.

- Any information I provide will be kept confidential to the researcher, the supervisors, and the transcriber.

- I understand that the results will be used for a PhD thesis, academic publications and presentation to conferences and Alliance teams or equivalent in New Zealand and overseas.

- Information provided by me may be used as direct quotes, however my name will not be used in reports, nor will any information that would identify me.

I would like a copy of the transcript of my interview

I would like a copy of the final thesis and any peer-reviewed published material

Signature of participant:

Name of participant:

Date:

Contact details:
Yes $\square \quad$ No

Yes $\square \quad$ No 
Framework for building capacity and capability for improvement in the New Zealand health system: a realist approach

\section{INFORMATION SHEET FOR CHIEF EXECUTIVES}

I would like to invite your staff to take part in this research. Please read this information before deciding whether you consent your staff taking part. Please confirm your consent by completing the consent form and emailing it to me (Kanchan.sharma@vuw.ac.nz). If you decide not to support, thank you for considering this request.

\section{What is the aim of the project?}

This research is about building capacity and capability for improvement in the New Zealand health system and thereby, shifting towards a learning system. The research is nested within the framing of complex adaptive systems. Small-scale change efforts at a micro level are insufficient to improve the performance and outcomes of the health system. LST initiatives that capitalise on the key elements of a complex adaptive system, such as the use of informal networks and distributed leadership, together with a deep understanding of the contexts that influence successful implementation of LST initiatives, are more likely to bring the different component parts of the system to work together successfully and sustainably.

By LST initiatives I am referring to co-ordinated, system-wide change involving multiple organisations that takes into consideration the relationships, interactions and behaviours of those in the system. The goal of LST initiatives is to stimulate communication and learning among different component parts of the system and for those in the system to understand the features of a complex adaptive system; thereby building the capacity and capability for improvement and shifting towards a learning system.

One such LST initiative is the System Level Measures (SLM) programme, which was developed with the sector leaders and clinicians. Alliances made up of District Health Board of domicile with their primary, hospital and community partners, are responsible for leading the implementation of the SLM programme.

This research aims to use the insights gained from the implementation of the SLM programme; first, to identify key elements that enable LST initiatives in the New Zealand health system; 
second, to identify how local context influence the key elements; and finally, to stimulate communication and learning within Alliances through the development and testing of a maturity matrix for the key elements.

\section{Who am I?}

My name is Kanchan Sharma and I am a Doctoral student in Faculty of Health at Victoria University of Wellington. I am also an employee of the $\mathrm{MoH}$, leading the SLM programme.

\section{Conflict of interest}

The inside knowledge and the first-hand experience with leading the implementation of the SLM programme gives me invaluable insights into the key elements that enable large system transformations in New Zealand and how the local contexts influence these elements.

However, my dual role as a Ministry employee and a researcher presents a conflict of interest that can be both real and perceived. It is therefore important for me to distinguish between the privileges I have from my position in respect to the research population and the genuine ethical issues that might arise, such as an impact on voluntary participation and the ability for participants to be honest in their feedback.

Participation in the research is voluntary. You and your staff do not have to participate if you do not want to and you do not have to give me a reason for your decision.

Data collected during this research will only be used for my thesis. I will not use the data collected for my Ministry role or disclose the data to any employee of the $\mathrm{MoH}$.

The workshops and the Alliance meetings will be facilitated by $\operatorname{Dr} X X X$, who is the SLM programme clinical lead and a PHO employee. This may also have a real and perceived conflict of interest.

Dr XXX has signed a confidentiality agreement to keep the identity of participants, and the information obtained from the workshops and meeting with Alliances confidential. XXX will not discuss the information obtained with anyone except with my supervisors, me, and potentially with the participants involved in the workshops and meeting.

The $\mathrm{MoH}$ may be interested in the information collected during this research or may in future use the output of the research, in particular the maturity matrix, in conjunction with the SLM programme. Any information shared with the $\mathrm{MoH}$ will be in accordance with the research 
confidentiality agreements. This means that data from the research will be combined and all identification information removed.

This research is using the insights from the SLM programme but is not undertaking a formal evaluation of the programme. If participants want to discuss or provide any feedback about the SLM programme, they should email Ministry directly (SLM@health.govt.nz).

This research has been approved by the Victoria University of Wellington Human Ethics Committee (application reference number 0000027356).

If I experience or am challenged by any conflicts of interest as the research proceeds, I will seek advice and support from my supervisors and the University Human Ethics Committee to appropriately manage it.

\section{How can you help?}

I would like to invite your staff to take part in the research because of their expertise in the health system or a complex system, its successes and challenges; their understanding of system improvement; and their potential involvement in the implementation of the SLM programme.

Supporting this research means you are allowing your staff to take part in the research during business hours and share their experiences relevant to the research questions. If your staff is invited to participate in the workshops, I request that your organisation pay for the travel costs of your staff to Wellington for two-day trips. Please indicate through the consent form if your organisation is able to pay for the travel costs.

\section{What will happen to the information you give?}

This research is confidential. Four data collection methods will be used.

1. Two workshops with selected senior leaders from the New Zealand health system. The researcher named below, research supervisors, and the workshop facilitator and the transcriber (both of whom will be required to sign a confidentiality agreement) will be aware of the identity of the study participants. The research data will be combined, and the participants' identity will not be revealed in any reports, presentations, or public documentation.

2. Interviews with selected senior leaders from international health systems, health consultancy firms and those leading other complex systems. The researcher named below, 
research supervisors, and the transcriber (who will be required to sign a confidentiality agreement) will be aware of the identity of the study participants. The research data will be combined, and the participants' identity will not be revealed in any reports, presentations, or public documentation.

3. Online survey with employees of DHBs and PHOs who have been involved in the implementation of the SLM programme - this will anonymous so participants cannot be identified.

4. Infield testing with Alliances. The researcher named below, research supervisors, and the facilitator and the transcriber (both of whom will be required to sign a confidentiality agreement) will be aware of the identity of the study participants. The research data will be combined, and the participants' identity will not be revealed in any reports, presentations, or public documentation.

Some of the information provided by the study participants may be used as direct quotes in the reports. However, all identification information will be removed.

The interview transcripts, summaries and any recordings will be kept securely and destroyed on 31 October 2022.

\section{What will the project produce?}

The information from my research will be used in my PhD thesis, academic publications and presentation to conferences and Alliance teams or equivalent in New Zealand and overseas.

The maturity matrix developed will be available to the Alliances to use it as a self-assessment tool to stimulate quality improvement by assessing their maturity on the key elements necessary to implement LST initiatives successfully and sustainably and facilitating communication and learning within the Alliances.

\section{If you support participation of your staff in this research: what will this involve?}

You do not have to support participation of your staff in this research if you don't want to. If you do decide to support, this will involve:

1. Two whole day attendance in workshops in Wellington on 21 and 28 August.

2. About 20 minutes of time for your staff involved in the implementation of the SLM programme to complete an anonymous online survey. 
You can ask any questions about the study at any time and be able to read any reports of this research by requesting a copy through the consent form.

All participants will be given the participant information sheet and written consents will be obtained for participation in workshops and Alliance meetings. The online survey is anonymous therefore participation in the survey implies consent.

The information shared by your employees during this research is confidential. Participants may share their experience but not reveal the identities or experiences of others. This will ensure anonymity of participants and their employing organisations.

If you have any questions or problems, who can you contact?

If you have any questions, either now or in the future, please feel free to contact me:

Student:

Name: Kanchan Sharma

University email address:

Kanchan.sharma@vuw.ac.nz
Supervisor:

Name: Professor Jackie Cumming

Role: Director, Health Services Research

Centre

School: Faculty of Health

Phone: 044636567

Jackie.cumming@vuw.ac.nz

\section{Human Ethics Committee information}

If you have any concerns about the ethical conduct of the research you may contact the Victoria University HEC Convenor: Dr Judith Loveridge. Email hec@vuw.ac.nz or telephone +64-4-463 6028. 
Framework for building capacity and capability for improvement in the New Zealand health system: a realist approach

\section{CHIEF EXECUTIVE CONSENT FOR THEIR EMPLOYEE TO PARTICIPATE IN THE RESEARCH}

This consent form will be held for three years.

Researcher: Kanchan Sharma, Faculty of Health, Victoria University of Wellington.

- I have read the Information Sheet and the project has been explained to me. My questions have been answered to my satisfaction. I understand that I can ask further questions at any time.

- I agree for my employees to be invited to participate in the research.

I understand that:

- I acknowledge that information shared by employees during the research is confidential. The employees may share their experience but not reveal the identities or experiences of others. Doing so may jeopardise the anonymity of participants and the employing organisations

- $\quad$ The identifiable information provided by participants will be destroyed on 31 October 2022.

- $\quad$ Any information provided by participants will be kept confidential to the researcher, the supervisor, the facilitator and the transcriber.

- I understand that the results will be used for a PhD thesis, academic publications and presentation to conferences and Alliance teams or equivalent in New Zealand and overseas.

- Information provided by participants may be used as direct quotes, however all identifiable information will be removed.

(for workshop participants only)

My organisation is able to pay for the travel costs of our staff to Wellington Yes $\square$ No for the two-day trips.

I would like a copy of the final thesis and any peer-reviewed published Yes No material

Signature of Chief Executive:

Name of organisation:

Date:

Contact details: 
Appendix 6: Workshop participant consent form

Framework for building capacity and capability for improvement in the New Zealand health system: a realist approach

\section{CONSENT TO PARTICIPATE IN WORKSHOP}

This consent form will be held for three years.

Researcher: Kanchan Sharma, Faculty of Health, Victoria University of Wellington.

- I have read the Information Sheet and the project has been explained to me. My questions have been answered to my satisfaction. I understand that I can ask further questions at any time.

- I agree to take part in an audio recorded workshop.

I understand that:

- I acknowledge that I am agreeing to keep the information shared during the workshop confidential. I am aware that after the workshop, I may share my experience but not reveal the identities or experiences of others. Doing so may jeopardise the anonymity of participants and the employing organisations.

- I can withdraw from the workshop while it is in progress however it will not be possible to withdraw the information I have provided up to that point as it will be part of a discussion with other participants.

- $\quad$ The identifiable information I have provided will be destroyed on 31 October 2022.

- Any information I provide will be kept confidential to the researcher, the supervisor, the workshop facilitator and the transcriber

- I understand that the results will be used for a PhD thesis, academic publications and presentation to conferences and Alliance teams or equivalent in New Zealand and overseas.

- Information provided by me may be used as direct quotes, however my name will not be used in reports, nor will any information that would identify me or my organisation.

I would like a copy of the final thesis and any peer-reviewed published material Yes No

Signature of participant:

Name of participant:

Date:

Contact details: 


\section{Workshop 1}

Date: 21 August 2019

Venue: Room RH103, Rutherford House - Bunny Street, Pipitea, Wellington

\begin{tabular}{|c|c|c|}
\hline Time & Task & Facilitator \\
\hline 0900 & Arrivals & \\
\hline 0930 & Introductions & $\operatorname{Dr} \mathrm{XXXX}$ \\
\hline 1000 & $\begin{array}{l}\text { Presentation (overview of the research, } \\
\text { insights from the System Level Measures (SLM) } \\
\text { programme and summary of the literature } \\
\text { findings) }\end{array}$ & Kanchan Sharma \\
\hline 1030 & Short Break & \\
\hline 1045 & $\begin{array}{l}\text { Session } 1 \text { - Reaching consensus on key } \\
\text { elements necessary for successful } \\
\text { implementation of LST initiatives }\end{array}$ & Participants \\
\hline 1200 & $\begin{array}{l}\text { Session } 2 \text { - Defining key elements (descriptions } \\
\text { and outcomes) }\end{array}$ & Participants \\
\hline 1230 & Lunch & \\
\hline 1300 & Session 2 continued & \\
\hline 1400 & $\begin{array}{l}\text { Session } 3 \text { - Identify local, national, social or } \\
\text { political circumstances that influence the key } \\
\text { elements, their effects and causes }\end{array}$ & Participants \\
\hline 1515 & Feedback from group activity & Group reps \\
\hline 1530 & Briefing for next workshop & Dr XXXX \\
\hline 1545 & Finish & \\
\hline
\end{tabular}


Appendix 8: Workshop two schedule

Workshop 2

Date: 28 August 2019

Venue: Room RH107 Rutherford House - Bunny Street, Pipitea, Wellington

\begin{tabular}{lll}
\hline Time & Task & Facilitator \\
\hline $\mathbf{0 9 0 0}$ & Arrivals & \\
\hline $\mathbf{0 9 3 0}$ & $\begin{array}{l}\text { Session 1 - Review of outcome descriptors from } \\
\text { first workshop and introduction to maturity matrix }\end{array}$ & $\begin{array}{l}\text { Dr XXX \& Kanchan } \\
\text { Sharma }\end{array}$ \\
\hline $\mathbf{1 0 3 0}$ & Break & \\
\hline $\mathbf{1 0 4 5}$ & Session 2 - Development of indicators & Dr XXX \\
\hline $\mathbf{1 1 4 5}$ & Session 3 - Construction of maturity matrix & Dr XXX \\
\hline $\mathbf{1 2 3 0}$ & Lunch & \\
\hline $\mathbf{1 3 0 0}$ & Session 3 continued & Dr XXX \\
\hline $\mathbf{1 4 3 0}$ & Finalising the maturity matrix & Dr XXX \\
\hline $\mathbf{1 5 1 5}$ & General discussion/feedback & Dr XXX \\
\hline $\mathbf{1 5 3 0}$ & Next steps & Kanchan Sharma \\
\hline $\mathbf{1 5 4 5}$ & Finish & \\
\hline
\end{tabular}




\section{Email to online survey potential participants}

Kia ora

My name is Kanchan Sharma and I am a Doctoral student in the Faculty of Health at the Victoria University of Wellington. I am also an employee of the MoH, leading the SLM programme.

I am undertaking a research titled:

Framework for building capacity and capability for improvement in the New Zealand health system: a realist approach

You are invited to take part in this research through an anonymous online survey. The survey will take you about 10 minutes to complete. Attached is the research information sheet that provides further information about the research, what your participation will involve, and how the information collected will be used.

Please read this information before deciding whether or not to take part. If you decide to participate, thank you. If you decide not to participate, thank you for considering this request.

Participation in the survey is anonymous. Please avoid using names of people or organisations in your response as these may potentially jeopardise anonymity of you and your organisation.

Please forward this email onto your colleagues in your organisation and district who have been or are involved in the implementation of the System Level Measures (SLM) programme to invite them to participate in the survey.

\section{Link to the survey}

Insert link

If you have any further questions, you may contact me through this email address Kanchan.sharma@vuw.ac.nz). 
Appendix 10: Alliance participant consent form

Framework for building capacity and capability for improvement in the New Zealand health system: a realist approach

\section{CONSENT TO PARTICIPATE IN THE ALLIANCE TESTING PROCESS}

This consent form will be held for three years.

Researcher: Kanchan Sharma, Faculty of Health, Victoria University of Wellington.

- I have read the Information Sheet and the project has been explained to me. My questions have been answered to my satisfaction. I understand that I can ask further questions at any time.

- I agree to take part in an audio recorded meeting.

I understand that:

- I acknowledge that I am agreeing to keep the information shared during the meeting confidential. I am aware that after the meeting, I may share my experience but not reveal the identities or experiences of others. Doing so may jeopardise the anonymity of participants and the employing organisations.

- I can withdraw from the meeting while it is in progress however it will not be possible to withdraw the information I have provided up to that point as it will be part of a discussion with other participants.

- $\quad$ The identifiable information I have provided will be destroyed on 31 October 2022.

- Any information I provide will be kept confidential to the researcher, the supervisor, the facilitator and the transcriber.

- I understand that the results will be used for a PhD thesis, academic publications and presentation to conferences and Alliance teams or equivalent in New Zealand and overseas.

- Information provided by me may be used as direct quotes, however my name will not be used in reports, nor will any information that would identify me.

I would like a copy of the final thesis and any peer-reviewed published Yes $\square \quad$ No material

Signature of participant:

Name of participant:

Date:

Contact details: 
Appendix 11: Facilitator confidentiality form

Workshop and Alliance Leadership Meeting FacilitatorConfidentialityAgreement

Project Title: Framework for building capacity and capability for improvement in the New Zealand health system: a realist approach

Principal Investigator: Kanchan M Sharma

I, agree to ensure that the information obtained during the workshops and the infield testing with the Alliances will remain confidential to Kanchan M Sharma, the research supervisors and myself.

I agree to take the following precautions:

1. I will ensure that no person, other than Kanchan M Sharma, the research supervisors and myself, hears or sees the data collected during the workshops and the infield testing with the Alliances.

2. I will not discuss any aspect of the data collection with anyone except Kanchan M Sharma, the research supervisors, and potentially with the participants.

Signature:

Date: 


\section{Transcribing Confidentiality Agreement}

Project Title: Framework for building capacity and capability for improvement in the New Zealand health system: a realist approach

Principal Investigator: Kanchan M Sharma

I, agree to ensure that the audiotapes I

transcribe will remain confidential to Kanchan M Sharma, the research supervisors and myself.

I agree to take the following precautions:

1. I will ensure that no person, other than Kanchan M Sharma, the research supervisors and myself hears the recording or sees the transcript.

2. I will ensure that no other person has access to my computer/device.

3. I will delete the files from my computer/device once the transcription has been completed.

4. I will not discuss any aspect of the recording with anyone except Kanchan M Sharma.

Signature:

Date: 


\section{REFERENCES}

1. Best A, Greenhalgh T, Lewis S, et al. Large-system transformation in health care: A realist review. The Milbank Quarterly 2012;90(3):421-56

2. Ministry of Health. Operational Policy Framework. 2020. https://nsfl.health.govt.nz/accountability/operational-policy-framework-0.

3. Evans J, Grudniewicz A, Baker G, et al. Organisational context and capabilities for integrating care: A framework for improvement. International Journal of Integrated Care 2016;16(3):1-14

4. Pawson R, Tilley N. Realistic evaluation. London: Sage Publications, 1997.

5. Sturmberg J. Health system redesign: How to make health care person-centred, equitable, and sustainable. Switzerland: Springer International Publishing, 2018.

6. Greenhalgh T, Papoutsi C. Studying complexity in health services research: Desperately seeking an overdue paradigm shift. BMC medicine 2018;16(1):95-95 doi: 10.1186/s12916-018-1089-4[published Online First: Epub Date]|.

7. Holden L. Complex adaptive systems: Concept analysis. Journal of Advanced Nursing 2005;52(6):651-57

8. Plsek $\mathrm{P}$, Wilson T. Complexity, leadership, and management in healthcare organisations. BMJ 2001;323(7315):746-49 doi: 10.1136/bmj.323.7315.746[published Online First: Epub Date]|.

9. Carmichael T, Hadžikadić M. The fundamentals of complex adaptive systems. In: Ted Carmichael, Andrew Collins, Mirsad Hadžikadić, eds. Complex adaptive systems: Views from the physical, natural, and social sciences. Switzerland: Springer International Publishing, 2019:1-16.

10. Ministry of Health. New Zealand Health System. 2018. https://www.health.govt.nz/newzealand-health-system.

11. Braithwaite J. Changing how we think about healthcare improvement. BMJ 2018;361:k2014 doi: 10.1136/bmj.k2014[published Online First: Epub Date]|.

12. Ministry of Health. About the Ministry. 2018. https://www.health.govt.nz/about-ministry.

13. Heimans J, Timms H. Understanding "New Power" The crowd is challenging traditional leadership. Here's how to harness energy. Harvard Business Review 2014;92(12):48-56

14. Ministry of Health. Achieving equity in health outcomes: Highlights of important national and international papers. 16 November 2018. 
https://www.health.govt.nz/publication/achieving-equity-health-outcomes-highlightsselected-papers.

15. Ministry of Health. System Level Measures Framework. 2018.

https://www.health.govt.nz/new-zealand-health-system/system-level-measuresframework? mega=NZ\%20health\%20system\&title=SLM.

16. Health Quality \& Safety Commission. About us. 2018. https://www.hqsc.govt.nz/about-us/.

17. World Health Organization. Framework on integrated, people-centred health services. Sixtyninth World Health Assembly. 2016.

http://www.who.int/servicedeliverysafety/areas/people-centred-care/framework/en/.

18. Ministry of Health. Longer, Healthier Lives: New Zealand's Health 1991-2017. 25 February 2020. https://www.health.govt.nz/system/files/documents/publications/longerhealthier-lives-new-zealands-health-1990-2017.pdf.

19. Deloitte. State of the State New Zealand 2016: Social investment for our future. New Zealand: New Zealand Institute of Economic Research.

20. Allen B, Eppel E. Holding on tight - NPM and the New Zealand performance improvement framework. Australian journal of public administration 2020;79(2):171-86 doi: 10.1111/1467-8500.12405[published Online First: Epub Date]|.

21. Cumming J. Integrated care in New Zealand. International journal of integrated care 2011;11(5) doi: 10.5334/ijic.678[published Online First: Epub Date]|.

22. Gauld R. New Zealand's post-2008 health system reforms: Toward re-centralization of organizational arrangements. Health policy 2012;106(2):110-3 doi: 10.1016/j.healthpol.2012.03.018[published Online First: Epub Date]|.

23. Health and Disability System Review. Health and Disability System Review - Final Report Pūrongo Whakamutunga. Wellington: HDSR, 2020.

24. Goodyear-Smith F, Ashton T. New Zealand health system: Universalism struggles with persisting inequities. The Lancet 2019;394(10196):432-42 doi: 10.1016/s01406736(19)31238-3[published Online First: Epub Date]|.

25. Menzies M, Middleton L. Using scenarios in public policy. Policy Quarterly 2020;16(2):41-49

26. Menzies M, Middleton L. Evaluating Health Futures in Aotearoa. World future review 2019;11(4):379-95 doi: 10.1177/1946756719862114[published Online First: Epub Date]|.

27. Institute of Medicine. Crossing the quality chasm: A new health system for the 21st century. Washington DC: National Academy Press, 2001. 
28. Plsek P, Greenhalgh T. The challenge of complexity in health care. BMJ 2001;323(7313):62528 doi: 10.1136/bmj.323.7313.625[published Online First: Epub Date]|.

29. Eppel E. Complexity thinking in public administration's theories-in-use. Public management review 2016;19(6):845-61 doi: 10.1080/14719037.2016.1235721[published Online First: Epub Date]|.

30. Perla J, Bradbury J, Gunther-Murphy J. Large-scale improvement initiatives in healthcare: A scan of the literature. Journal For Healthcare Quality 2013;35(1):30-40 doi:

10.1111/j.1945-1474.2011.00164.x[published Online First: Epub Date]|.

31. Doebbeling B, Flanagan M. Emerging perspectives on transforming the healthcare system. Medical Care 2011;49(12 Suppl 1):S59-S64

32. NHS England. Leading large scale change: A practical guide. 2018.

https://www.england.nhs.uk/wp-content/uploads/2017/09/practical-guide-large-scalechange-april-2018-smll.pdf.

33. Khan S, Vandermorris A, Shepherd J, et al. Embracing uncertainty, managing complexity: Applying complexity thinking principles to transformation efforts in healthcare systems. BMC Health Serv Res 2018;18(1):192 doi: 10.1186/s12913-018-2994-0[published Online First: Epub Date]|.

34. McDaniel R, Driebe D, Lanham $H$. Health care organisations as complex systems: New perspectives on design and management. In: Leonard H Friedman et al., ed. Annual Review of Health Care Management: Revisiting the Evolution of Health Systems Organisation. ProQuest Ebook Central: Emerald Publishing Ltd, 2013:3-26.

35. Bevan $\mathrm{H}$, Fairman $\mathrm{S}$. The new era of thinking and practice in change and transformation. United Kingdom: NHS Improving quality 2017.

36. Obucina M, Harris N, Fitzgerald J, et al. The application of Triple Aim framework in the context of primary healthcare: A systematic literature review. Health policy 2018;122(8):900-07 doi: 10.1016/j.healthpol.2018.06.006[published Online First: Epub Date]|.

37. Urtaran-Laresgoiti M, Alvarez-Rosete A, Nuno-Solinis R. A system-wide transformation towards integrated care in the Basque Country: A realist evaluation. International Journal of Care Co-ordination 2018;21(3):98-108

38. Martin L, Nelson E, Lloyd R, et al. Whole system measures: IHI innovation series white paper. Cambridge, Massachusetts: Institute for Healthcare Improvement, 2007. 
39. Ministry of Health. Integrated Performance and Incentive Programme Expert Advisory Report Final. February 2014. https://www.hiirc.org.nz/page/45527/expert-advisorygroup-eag-report-on-the-integrated/?q=ipif\&highlight=ipif\&section=35484.

40. Ministry of Health. Guide to using the System Level Measures Framework for quality improvement. May 2018. https://nsfl.health.govt.nz/dhb-planning-package/systemlevel-measures-framework/system-level-measures-annual-plan-guidance.

41. Chalmers L, Ashton T, Tenbensel T. Measuring and managing health system performance: An update from New Zealand. Health policy 2017;121(8):831-35 doi: 10.1016/j.healthpol.2017.05.012[published Online First: Epub Date]|.

42. Ministry of Health. Alliancing charter. 2018. https://nsfl.health.govt.nz/dhb-planningpackage/system-level-measures-framework/system-level-measures-annual-planguidance.

43. Gauld R. The theory and practice of integrative health care governance. Journal of Integrated Care 2017;25(1):61-72 doi: 10.1108/JICA-10-2016-0035[published Online First: Epub Date]|.

44. Bartlett M, Basten R, McKinley R. Green shoots of recovery: A realist evaluation of a team to support change in general practice. BMJ Open 2017;7(2):e014165 doi: 10.1136/bmjopen-2016-014165[published Online First: Epub Date]|.

45. Gordon A, Goodman C, Davies S, et al. Optimal healthcare delivery to care homes in the UK: A realist evaluation of what supports effective working to improve healthcare outcomes. Age and ageing 2018 doi: 10.1093/ageing/afx195[published Online First: Epub Date]|.

46. Gossip K, Gouda H, Lee Y, et al. Monitoring and evaluation of disaster response efforts undertaken by local health departments: A rapid realist review. BMC Health Serv Res 2017;17(1):450 doi: 10.1186/s12913-017-2396-8[published Online First: Epub Date]|

47. Westhorp G. Realist impact evaluation: An introduction. September 2014. https://www.odi.org/publications/8716-realist-impact-evaluation-introduction.

48. Marchal B, van Belle S, van Olmen J, et al. Is realist evaluation keeping its promise? A review of published empirical studies in the field of health systems research. Evaluation 2012;18(2):192-212 doi: 10.1177/1356389012442444[published Online First: Epub Date]|.

49. Manzano A. The craft of interviewing in realist evaluation. Evaluation 2016;22(3):342-60 doi: 10.1177/1356389016638615[published Online First: Epub Date]|. 
50. Gill D. The iron cage recreated: The performance management of state organisations in New Zealand. Wellington: Institute of Policy Studies, 2011.

51. Cook L, Huges R. Information and institutions of government accountability. New Zealand: Hughes Books, 2018.

52. Electoral Commission. What is New Zealand's system of government? https://elections.nz/democracy-in-nz/what-is-new-zealands-system-of-government/.

53. Buse K, Mays N, Walt G. Making health policy. Maidenhead, UNITED KINGDOM: McGrawHill Education, 2012.

54. Gauld R. Questions about New Zealand's health system in 2013, its 75th anniversary year. NZMJ 2013;126(1380):68-74

55. Cumming J, McDonald J, Barr C, et al. New Zealand health system review. Health systems in transition. Geneva: World Health Organization, 2014.

56. Tenbensel T, Ashton T. New Zealand's healthcare system: Rapid reform and incremental change. Health reforms across the world: World Scientific Publishing Co Pte Ltd, 2020:249-73.

57. Accident Compensation Corporation. About ACC. 2020. https://www.acc.co.nz/about-us/

58. Gauld R. Revolving doors: New Zealand's health reforms, the continuing saga. 2 nd ed. ed. Wellington [N.Z.]: Health Services Research Centre, 2009.

59. Cumming J. Commissioning in New Zealand: learning from the past and present. Australian Journal of Primary Health 2016;22(1):34-39 doi: 10.1071/PY15164[published Online First: Epub Date]|.

60. Gauld R. Could lessons have been drawn from New Zealand for England's NHS reforms? Commonwealth \& Comparative Politics 2016;54(4):518-35 doi: 10.1080/14662043.2016.1228151[published Online First: Epub Date]|.

61. New Zealand Public Health and Disability Act. New Zealand, 2000.

62. World Health Organization. Alma-Ata Declaration on Primary Healthcare: Report of the International Conference on Primary Health Care. Geneva: World Health Organization, 1978.

63. PHO Services Agreement: Version 7.2. 1 July 2020. https://tas.health.nz/assets/Uploads/PHO-Services-Agreement-Version-7.2-1-July2020.pdf.

64. Smith J, Cumming J. Where next for primary health organisations in New Zealand? Wellington: HSRC, Victoria University of Wellington, 2009. 
65. Smith J, McDonald J, Cumming J. Developing and implementing high impact changes for primary health care in New Zealand - Discussion document prepared for District Health Boards New Zealand. Wellington: HSRC, Victoria University of Wellington, 2008.

66. Ministry of Health. Overview of the health system. 2017. https://www.health.govt.nz/newzealand-health-system/overview-health-system.

67. Aunger JA, Millar R, Greenhalgh J, et al. Why do some inter-organisational collaborations in healthcare work when others do not? A realist review. Systematic reviews 2021;10(1):82 doi: 10.1186/s13643-021-01630-8[published Online First: Epub Date]|.

68. Timmins N, Ham C. The quest for integrated health and social care - A case study in Canterbury, New Zealand: The King's Fund, 2013.

69. Serra M, Ospina-Palacio D, Espinal S, et al. Trusted networks: The key to achieve world-class health outcomes on a shoestring. Bogota, Columbia: Beatin Inc., 2018.

70. Holmes B, Best A, Davies $\mathrm{H}$, et al. Mobilising knowledge in complex health systems: A call to action. Evidence \& Policy: A Journal of Research, Debate and Practice 2017;13(3):539-60 doi: 10.1332/174426416x14712553750311[published Online First: Epub Date]|.

71. Lopez-Duarte C, Vidal-Suarez M. Handbook on international alliance and network research. Cheltenham: Edward Elgar Pub. Ltd., 2015.

72. Charles A. Integrated Care Systems explained: Making sense of systems, places and neighbourhoods. April 2020. https://www.kingsfund.org.uk/publications/integratedcare-systems-explained.

73. Shortell S, McCurdy R. Integrated health systems. In: W B Rouse, D A Cortese, eds. Engineering the system of healthcare delivery. The Netherlands: IOS Press, 2010.

74. NHS Confederation - Integrated Care Systems Network. From place-based to place-led: A whole-area approach to integrating care systems. London: NHS Confederation, 2020.

75. Pratt J, Gordon P, Plamping D. Working whole systems: Putting theory into practice in organisations. 2nd ed. United Kingdom: Radcliffe Publishing Ltd, 2005.

76. Ministry of Health. Health alliances. 19 March 2011. https://www.health.govt.nz/newzealand-health-system/key-health-sector-organisations-and-people/health-alliances.

77. Matheson A, Bourke C, Verhoeven A, et al. Lowering hospital walls to achieve health equity. BMJ 2018;362:k3597 doi: 10.1136/bmj.k3597[published Online First: Epub Date]|.

78. Hernandez A, Ruano A, Marchal B, et al. Engaging with complexity to improve the health of indigenous people: A call for the use of systems thinking to tackle health inequity. 
International journal for equity in health 2017;16(1):26 doi: 10.1186/s12939-017-05212[published Online First: Epub Date]|.

79. Hood C. A public management for all seasons? Public Administration 1991;69(Spring):3-19

80. O'Flynn J. From New Public Management to Public Value: Paradigmatic change and managerial implications. Australian Journal of Public Administration 2007;66(3):353-66 doi: 10.1111/j.1467-8500.2007.00545.x[published Online First: Epub Date]|.

81. Ryan B, Gill D. Future state: Directions for public management in New Zealand. Wellington: Victoria University Press, 2011.

82. Allen B, Berman E, Clara C, et al. Independent review of the Performance Improvement Framework - overview and key messages: School of Government, Victoria University of Wellington, 2017.

83. Pye S. PHO Performance Programme : Six years on. Best practice (Dunedin, N.Z.) 2011(41):40-49

84. Ministry of Health. Health Targets. 2018. https://www.health.govt.nz/new-zealand-healthsystem/health-targets?mega=NZ\%20health\%20system\&title=Health\%20targets.

85. Ministry of Health. How is my DHB performing? 2018. https://www.health.govt.nz/newzealand-health-system/health-targets/how-my-dhb-performing-2017-18.

86. Ministry of Health. How is my PHO performing? 2018. https://www.health.govt.nz/newzealand-health-system/health-targets/how-my-pho-performing.

87. Gillett G. Justice and health care in a caring society. BMJ 1998;317:53-54

88. Gauld R, Burgers J, Dobrow M, et al. Healthcare system performance improvement: A comparison of key policies in seven high-income countries. Journal of health organization and management 2014;28(1):2-20 doi: 10.1108/JHOM-03-20130057[published Online First: Epub Date]|.

89. Tenbensel T, Chalmers L, Willing E. Comparing the implementation consequences of the immunisation and emergency department health targets in New Zealand. Journal of health organization and management 2016;30(6):1009-24 doi: 10.1108/JHOM-08-20150126[published Online First: Epub Date]|.

90. Tenbensel T, Chalmers L, Jones $P$, et al. New Zealand's emergency department target - did it reduce ED length of stay, and if so, how and when? BMC Health Services Research 2017;17(1):1-15 doi: 10.1186/s12913-017-2617-1[published Online First: Epub Date]|.

91. Ministry for Culture and Heritage. The Treaty in brief. 2017.

https://nzhistory.govt.nz/politics/treaty/the-treaty-in-brief. 
92. Ministry of Health. He Korowai Oranga: Māori Health Strategy. In: Ministry of Health, ed. Wellington, New Zealand, 2002.

93. Cram F, Te Huia B, Te Huia T, et al. Oranga and Māori health inequities, 1769-1992. Wellington: Ministry of Health, 2019.

94. Durie M. Indigenizing mental health services: New Zealand experience. Transcultural Psychiatry 2011;48(1-2):24-36 doi: 10.1177/1363461510383182[published Online First: Epub Date]|.

95. Smith G. Beyond political literacy: From conscientization to transformative praxis. Counterpoints 2005;275:29-42

96. Health and Disability System Review. Health and Disability System Review - Interim Report. Hauora Manaaki ki Aotearoa Whānui - Pūrongo mō Tēneī Wā. Wellington: HDSR, 2019.

97. Came H, McCreanor T, Manson L. Upholding Te Tiriti, ending institutional racism and Crown inaction on health equity. The New Zealand medical journal 2019;132(1492):61-66

98. Sheridan N, Hand J. Inequity, indigeneity and progressive politics in Aotearoa / New Zealand. Race, gender \& class (Towson, Md.) 2011;18(3/4):177-90

99. Matheson A. Health inequality as a large-scale outcome of complex social systems: Lessons for action on the Sustainable Development Goals. International journal of environmental research and public health 2020;17(8):2684 doi:

10.3390/ijerph17082684[published Online First: Epub Date]|.

100. Statistics New Zealand. New Zealand period life table: 2012-14. 8 May 2015.

https://www.stats.govt.nz/information-releases/new-zealand-period-life-tables201214.

101. Waitangi Tribunal. Report on Stage One of the Health Services and Outcomes Kaupapa Inquiry. New Zealand: Waitangi Tribunal WAI 2575, 2019.

102. Waitangi Tribunal. Waitangi Tribunal: Te Rōpū Whakamana i te Tiriti o Waitangi. 2020. https://waitangitribunal.govt.nz/.

103. Ministry of Health. Whakamaua: Māori Health Action Plan 2020-2025. 17 September 2020. https://www.health.govt.nz/publication/whakamaua-maori-health-action-plan2020-2025.

104. OECD. Health expenditure and financing. 2020. https://stats.oecd.org/Index.aspx?DataSetCode=SHA.

105. The Treasury. Vote Health - The Estimates of Appropriations 2019/20 - Health Sector 2019. https://www.treasury.govt.nz/sites/default/files/2019-05/est19-v6-health.pdf. 
106. New Zealand Government. District Health Boards financial performance to 2016 and 2017 plans, February 2017.

107. The Treasury. Financial statements of the Government of New Zealand for the year ended 30 June 2019. 8 October 2019. https://www.treasury.govt.nz/publications/yearend/financial-statements-2019.

108. Keene L, Bagshaw P, Nicholls MG, et al. Funding New Zealand's public healthcare system: Time for an honest appraisal and public debate. The New Zealand Medical Journal (Online) 2016;129(1435):10-20

109. Schneider E, Sarnak D, Squires D, et al. Mirror, Mirror 2017: International comparisons reflects flaws and opportunities for better US health care. In: Fund TC, ed.: The Commonwealth Fund, 2017.

110. Cumming J. New Zealand: Strengthening primary healthcare. In: Jeffrey Braithwaite, Russell Mannion, Yukihiro Matsuyama, et al., eds. Healthcare systems: Future predictions for global care. 1 ed. Boca Raton: CRC Press, 2018:421-26.

111. New Zealand Government. Health loss in New Zealand 1990-2013: A report from the New Zealand burden of diseases, injuries and risk factors study. In: Ministry of Health, ed. New Zealand, 2016.

112. Walsh M, Gray C. The contribution of avoidable mortality to the life expectancy gap in Māori and Pacific populations in New Zealand - a decomposition analysis. New Zealand medical journal 2019;132(1492):46-60

113. Health Quality \& Safety Commission. Primary care patient experience survey: Results from the first year of pilots. 2017. https://www.hqsc.govt.nz/our-programmes/healthquality-evaluation/publications-and-resources/publication/3161/.

114. Ministry of Health. Annual update of key results 2018/19: New Zealand Health Survey. November 2019. https://www.health.govt.nz/publication/annual-update-key-results2018-19-new-zealand-health-survey.

115. Ministry of Health. New Zealand Health Strategy: Future direction. 2016. https://www.health.govt.nz/publication/new-zealand-health-strategy-2016.

116. New Zealand Government. The Primary Health Care Strategy. In: Ministry of Health, ed. Wellington, New Zealand, 2001.

117. Ministry of Health. DHB sector financial reports 2018-19. 21 February 2020. https://www.health.govt.nz/new-zealand-health-system/key-health-sector- 
organisations-and-people/district-health-boards/accountability-and-funding/summaryfinancial-reports/dhb-sector-financial-reports-2018-19.

118. Health and Disability System Review. Terms of Reference. December 2018.

https://systemreview.health.govt.nz/about/terms-of-reference/.

119. Gauld R. The new health policy. England: Open University Press, 2009.

120. Doolan-Noble F, Lyndon M, Hau S, et al. How well does your healthcare system perform?

Tracking progress toward the triple aim using system level measures. NZMJ 2015;128(No 1415)

121. Tenbensel T, Silwal PR, Walton L, et al. New Zealand's Integration- Based Policy for Driving Local Health System Improvement - Which Conditions Underpin More Successful Implementation? International Journal of Integrated Care 2021;21(2):8 doi: 10.5334/ijic.5602[published Online First: Epub Date]|.

122. Baker G, Axler R. Creating a high performing healthcare system for Ontario: Evidence supporting strategic changes in Ontario: University of Toronto, 2015.

123. Kelly B, Perkins DF. Handbook of Implementation Science for Psychology in Education. New York: Cambridge University Press, 2012.

124. Sheingold B, Hahn J. The history of healthcare quality: The first 100 years 1860-1960. International Journal of Africa Nursing Sciences 2014;1:18-22 doi: https://doi.org/10.1016/j.ijans.2014.05.002[published Online First: Epub Date]|.

125. Petrie D, Swanson RC. The mental demands of leadership in complex adaptive systems. Healthcare management forum 2018;31(5):206-13 doi: 10.1177/0840470418778051[published Online First: Epub Date]|.

126. Lipsitz L. Understanding health care as a complex system: The foundation for unintended consequences. JAMA 2012;308(3):243-4 doi: 10.1001/jama.2012.7551[published Online First: Epub Date]|.

127. Martin C. Complex adaptive systems approaches in health care: A slow but real emergence? Journal of Evaluation in Clinical Practice 2018;24(1):266-68 doi: 10.1111/jep.12878[published Online First: Epub Date]|.

128. The Health Foundation. Evidence scan: Complex adaptive systems: The Health Foundation, August 2010.

129. World Health Organization. Everybody's business: Strengthening health systems to improve health outcomes: WHO's framework for action. Geneva: World Health Organization, 2007. 
130. Petticrew M, Knai C, Thomas J, et al. Implications of a complexity perspective for systematic reviews and guideline development in health decision making. BMJ Global Health 2019;4(Suppl 1):e000899 doi: 10.1136/bmjgh-2018-000899[published Online First: Epub Date]|.

131. Alliance for Health Policy and Systems Research. Systems thinking for health systems strengthening. Geneva: World Health Organization, 2009.

132. Martin C, Félix-Bortolotti M. W(h)ither complexity? The emperor's new toolkit? Or elucidating the evolution of health systems knowledge? Journal of Evaluation in Clinical Practice 2010;16(3):415-20 doi: 10.1111/j.1365-2753.2010.01461.x[published Online First: Epub Date]|.

133. Hugh A, Anna C, Bryan J, et al. Making the case for quality improvement: Lessons for NHS boards and leaders: The King's Fund, October 2017.

134. James BC, Savitz LA. How Intermountain trimmed health care costs through robust quality improvement efforts. Health affairs 2011;30(6):1185-91 doi: 10.1377/hlthaff.2011.0358[published Online First: Epub Date]|.

135. Estabrooks C. Engagement-capable environments: No less challenging than other large system changes. Healthcare Papers 2017;17(2):40-45

136. Anderson R. Commentary on "Health care organisations as complex systems: New perspectives on design and management" by Reuben R McDaniel, Dean J Driebe, and Holly Jordan Lanham. In: Leonard H Friedman et al., ed. Annual Review of Health Care Management: Revisiting the Evolution of Health Systems Organisation. ProQuest Ebook Central: Emerald Publishing Ltd, 2013:27-36.

137. The Health Foundation. Effective networks for improvement: Developing and managing effective networks to support quality improvement in healthcare. London, March 2014.

138. Braithwaite J, Runciman W, Merry A. Towards safer, better healthcare: Harnessing the natural properties of complex sociotechnical systems. Quality \& safety in health care 2009;18(1):37-41 doi: 10.1136/qshc.2007.023317[published Online First: Epub Date]|.

139. Berwick D. The science of improvement. JAMA 2008;299(10):1182 doi:

10.1001/jama.299.10.1182[published Online First: Epub Date]|.

140. Matheson A, Walton M, Gray R, et al. Evaluating a community-based public health intervention using a complex systems approach. Journal of public health (Oxford, England) 2018;40(3):606-13 doi: 10.1093/pubmed/fdx117[published Online First: Epub Date]|. 
141. Matheson A, Walton M, Gray R, et al. Strengthening prevention in communities through systems change: Lessons from the evaluation of Healthy Families NZ. Health promotion international 2019;35(5):947-57 doi: 10.1093/heapro/daz092[published Online First: Epub Date]|.

142. Stouten J, Rousseau D, De Cremer D. Successful organizational change: Integrating the management practice and scholarly literatures. Academy of Management Annals 2018;12(2) doi: 10.5465/annals.2016.0095[published Online First: Epub Date]|.

143. Blackman D, Halligan J, O'Flynn J. Crossing Boundaries in Public Management and Policy: The International Experience. London, UNITED KINGDOM: Taylor \& Francis Group, 2013.

144. Grudniewicz A, Tenbensel T, Evans J, et al. 'Complexity-compatible' policy for integrated care? Lessons from the implementation of Ontario's Health Links. Social science \& medicine (1982) 2018;198:95-102 doi: 10.1016/j.socscimed.2017.12.029[published Online First: Epub Date]|.

145. Strategy\&. Cultures role in enabling organizational change - Survey ties transformation success to deft handling of cultural issues: Booz \& Company, 2013.

146. Westrum R. A typology of organisational cultures. J Quality and Safety in Health Care 2004;13(suppl 2):ii22-ii27 doi: 10.1136/qshc.2003.009522 \%[published Online First: Epub Date]|.

147. Kirk S. How's your DHB doing? Govt does away with national health targets. Stuff News 201826 June.

148. Chiang J. Health experts divided over changes to national targets. Radio New Zealand 2018 28 June.

149. Bennett L. National warns of more deaths with no public health targets. New Zealand Herald 201826 June

150. Rhydderch M. Organisational change theory and the use of indicators in general practice. Quality and Safety in Health Care 2004;13(3):213-17 doi: 10.1136/qshc.2003.006536[published Online First: Epub Date]|.

151. Edwards A, Rhydderch $M$, Engels $Y$, et al. Assessing organisational development in European primary care using a group-based method: A feasibility study of the Maturity Matrix. International Journal of Health Care Quality Assurance 2010;23(1):8-21

152. Buch M, Edwards A, Eriksson T. Participants' evaluation of a group-based organisational assessment tool in Danish general practice: The Maturity Matrix. Quality in Primary Care 2009;17(5):311-22 
153. Elwyn G, Rhydderch M, Edwards A, et al. Assessing organisational development in primary medical care using a group based assessment: The Maturity Matrix. Quality \& safety in health care 2004;13(4):287-94

154. Elwyn G, Bekkers M, Tapp L, et al. Facilitating organisational development using a groupbased formative assessment and benchmarking method: Design and implementation of the International Family Practice Maturity Matrix. Quality \& safety in health care 2010;19(6):e48

155. Kirk M, Simpson A, Llewellyn M, et al. Evaluating the role of Cardiac Genetics Nurses in inherited cardiac conditions services using a Maturity Matrix. European Journal of Cardiovascular Nursing 2014;13(5):418-28

156. Yousefi V, Evans M. Hospital medicine maturity framework: Proposing a novel framework for defining stages of hospital medicine in Canada. Healthcare Quarterly 2011;14(4):6773

157. Lombarts $M$, Rupp I, Vallejo P, et al. Differentiating between hospitals according to the "maturity" of quality improvement systems: A new classification scheme in a sample of European hospitals. Quality \& safety in health care 2009;18 Suppl 1:i38-43

158. Ramadan N, Arafeh M. Healthcare quality maturity assessment model based on quality drivers. International Journal of Health Care Quality Assurance 2016;29(3):337-50

159. Eriksson T, Siersma V, Logstrup L, et al. Documenting organisational development in general practice using a group-based assessment method: The Maturity Matrix. Quality \& safety in health care 2010;19(5):e37

160. Storm I, Harting J, Stronks K, et al. Measuring stages of health in all policies on a local level: The applicability of a maturity model. Health policy 2014;114(2-3):183-91

161. Greenhalgh T, Wherton J, Papoutsi C, et al. Beyond Adoption: A New Framework for Theorizing and Evaluating Nonadoption, Abandonment, and Challenges to the Scale-Up, Spread, and Sustainability of Health and Care Technologies. Journal of medical Internet research 2017;19(11):e367 doi: 10.2196/jmir.8775[published Online First: Epub Date]|.

162. Minkman M, Vat L. A self-evaluation tool for integrated care services: the Development Model for Integrated Care applied in practice. International Journal of Integrated Care 2012;12(Suppl3)(e156)

163. SCIROCCO - Scaling Integrated Care in Context. SCIROCCO Maturity Model for Integrated Care. 2016. https://www.scirocco-project.eu/. 
164. Grooten L, Vrijhoef H, Calciolari S, et al. Assessing the maturity of the healthcare system for integrated care: Testing measurement properties of the SCIROCCO tool. BMC medical research methodology 2019;19(1):63 doi: 10.1186/s12874-019-07041[published Online First: Epub Date]|.

165. Kirst M, Im J, Burns T, et al. What works in implementation of integrated care programs for older adults with complex needs? A realist review. International Journal for Quality in Health Care 2017;29(5):612-24 doi: 10.1093/intqhc/mzx095[published Online First: Epub Date]|.

166. Flynn R, Newton A, Rotter T, et al. The sustainability of lean in pediatric healthcare: A realist review. Systematic reviews 2018;7(1):137 doi: 10.1186/s13643-018-0800z[published Online First: Epub Date]|.

167. Sobh R, Perry C. Research design and data analysis in realism research. European Journal of Marketing 2006;40(11/12):1194-209 doi: 10.1108/03090560610702777[published Online First: Epub Date]|.

168. Westhorp G. Using complexity-consistent theory for evaluating complex systems. Evaluation 2012;18(4):405-20 doi: 10.1177/1356389012460963[published Online First: Epub Date]|.

169. Mertens D. Chapter 1: An introduction to research. Research and evaluation in education and psychology. Fourth ed. United States of America: SAGE Publications, Inc., 2015.

170. Pawson R. Evidence-based policy: A realist perspective. London: Sage Publications, 2006.

171. Pawson R. The science of evaluation: A realist manifesto. London: Sage Publications, 2013.

172. Hewitt G, Sims S, Harris R. The realist approach to evaluation research: An introduction. International Journal of Therapy and Rehabilitation May 2012;19(5):250-59

173. Patton M. Qualitative research \& evaluation methods. Fourth ed. United States of America: Sage Publications, 2015.

174. Emmel N, Greenhalgh J, Manzano A, et al., editors. Doing realist research. London: SAGE Publications, 2018.

175. Shaw J, Steele Gray C, Baker G, et al. Mechanisms, contexts and points of contention: Operationalizing realist-informed research for complex health interventions. BMC medical research methodology 2018;18(1):178-78 doi: 10.1186/s12874-018-06414[published Online First: Epub Date] |.

176. Shearn K, Allmark P, Piercy H, et al. Building realist program theory for large complex and messy interventions. International Journal of Qualitative Methods 
2017;16(1):1609406917741796 doi: 10.1177/1609406917741796[published Online First: Epub Date]|.

177. Wong G, Westhorp G, Manzano A, et al. RAMESES II reporting standards for realist evaluations. BMC Med 2016;14(1):96 doi: 10.1186/s12916-016-0643-1[published Online First: Epub Date]|.

178. Greenhalgh. T, Humphrey. C, Hughes. J, et al. How do you modernise a health service? A realist evaluation of whole-scale transformation in London. The Milbank Quarterly 2009;87(2):391-416

179. Jagosh J, Bush P, Salsberg J, et al. A realist evaluation of community-based participatory research: Partnership synergy, trust building and related ripple effects. BMC public health 2015;15(1):725 doi: 10.1186/s12889-015-1949-1[published Online First: Epub Date]|.

180. Chen H. Evaluation versus research. In: Frey BB, ed. The SAGE encyclopedia of educational research, measurement, and evaluation. Thousand Oaks: SAGE Publications, Inc., 2018.

181. Clarke A. Understanding evaluation. SAGE research methods. London: SAGE Publications Ltd, 1999.

182. Marchal B. Why do some hospitals perform better than others? A realist evaluation of the role of health workforce management in well performing health care organisations. A study of 4 hospitals in Ghana [PhD]. University of Brussels, 2011.

183. The RAMESES II Project. Retroduction in realist evaluation. 2017. http://www.ramesesproject.org/media/RAMESES || Retroduction.pdf.

184. Pavelin K, Pundir S, Cham JA. Ten simple rules for running interactive workshops. PLoS computational biology 2014;10(2):e1003485 doi: 10.1371/journal.pcbi.1003485[published Online First: Epub Date]|.

185. Sweeney M. Cooperative relational strategies: Storm and sort. Wellington, New Zealand: Impact Education Group, 2014.

186. Kagan S. Cooperative learning. California: Kagan Publishing, 1994.

187. Tuten T. Conducting online surveys. In: Samuel Gosling, John Johnson, eds. Advanced methods for conducting online behavioral research. Washington: American Psychological Association, American Psychological Association, Washington, DC, 2010:179-92, Chapter vii, 286 Pages.

188. Ministry of Health. My DHB. 2019. https://www.health.govt.nz/new-zealand-healthsystem/my-dhb. 
189. Ministry of Health. A Guide to the Commissioning Framework for Mental Health and Addiction 2015: Consultation document. Wellington: Ministry of Health, 2015.

190. McGrath K, Bennett D, Ben-Tovim D, et al. Implementing and sustaining transformational change in health care: Lessons learnt about clinical process redesign. MJA 2008;188:S32S35

191. Gauld R, Horsburgh S, Flynn M, et al. Do different approaches to clinical governance development and implementation make a difference? Findings from Ireland and New Zealand. Journal of Health, Organisation and Management 2017;31(7/8):682-95

192. NHS Scotland. Joint Strategic Commissioning - A Definition: Joint Strategic Commissioning across adult health and social care. Scotland: The Scottish Government, 2012.

193. Ministry of Health. Achieving equity. 2019. https://www.health.govt.nz/aboutministry/what-we-do/work-programme-2018/achieving-equity.

194. Lukas CV, Holmes SK, Cohen AB, et al. Transformational change in health care system: An organisational model. Health Care Management Review 2007;32(4):309-20

195. Te Arawhiti - The Office for Māori Crown Relations. Engagement framework: Crown engagement with Māori. 1 October 2018. https://tearawhiti.govt.nz/assets/MaoriCrown-Relations-Roopu/451100e49c/Engagement-Framework-1-Oct-18.pdf.

196. Bolden R. Distributed leadership in organizations: A review of theory and research. International Journal of Management Reviews 2011;13(3):251-69 doi: 10.1111/j.14682370.2011.00306.x[published Online First: Epub Date]|.

197. Gronn P. Distributed leadership as a unit of analysis. The Leadership Quarterly 2002;13(4):423-51 doi: https://doi.org/10.1016/S1048-9843(02)00120-0[published Online First: Epub Date]|.

198. Willis C, Saul J, Bevan H, et al. Sustaining organizational culture change in health systems. Journal of health organization and management 2016;30(1):2-30 doi: 10.1108/JHOM07-2014-0117[published Online First: Epub Date]|.

199. Berta W, Virani T, Bajnok I, et al. Understanding whole systems change in health care: Insights into system level diffusion from nursing service delivery innovations: A multiple case study. Evidence \& Policy: A Journal of Research, Debate and Practice 2014;10(3):313-36 doi: 10.1332/174426413X662798[published Online First: Epub Date]|.

200. Taylor N, Clay-Williams R, Hogden E, et al. High performing hospitals: A qualitative systematic review of associated factors and practical strategies for improvement. BMC 
Health Serv Res 2015;15:244 doi: 10.1186/s12913-015-0879-z[published Online First: Epub Date]|.

201. Ansell C, Sørensen E, Torfing J. Improving policy implementation through collaborative policymaking. Policy and Politics 2017;45(3):467-86 doi: http://dx.doi.org/10.1332/030557317X14972799760260[published Online First: Epub Date]|.

202. Kidd J, Came H, Doole C, et al. A critical analysis of te Tiriti o Waitangi application in primary health organisations in Aotearoa New Zealand: findings from a nationwide survey. Health Soc Care Community 2021; 00. https://onlinelibrary.wiley.com/doi/pdf/10.1111/hsc.13417.

203. Steele Gray C, Wodchis W, Baker G, et al. Mapping for conceptual clarity: Exploring implementation of integrated community-based primary health care from a whole system perspective. International journal of integrated care 2017;17(5):143 doi: 10.5334/ijic.3451[published Online First: Epub Date]|.

204. Eppel E, Gill D, Lips M, et al. The cross-organizational collaboration solution? Conditions, roles and dynamics in New Zealand. In: Janine O'Flynn, Deborah Blackman, John Halligan, eds. Crossing boundaries in Public Management and Policy. 1st ed. London: Routledge, 2014:67-83.

205. Fawkes S. Leadership for systems change in preventive health: Review of the literature and current activity. Melbourne: Victorian Government Department of Health, 2012.

206. Uhl-Bien M, Marion R, McKelvey B. Complexity leadership theory: Shifting leadership from the industrial age to the knowledge era. The Leadership Quarterly 2007;18(4):298-318 doi: 10.1016/j.leaqua.2007.04.002[published Online First: Epub Date]|.

207. Chunharas S, Davies DSC. Leadership in health systems: A new agenda for interactive leadership. Health Systems \& Reform: Effective Leadership for Health Systems, sponsored by the Alliance for Health Policy and Systems Research 2016;2(3):176-78 doi: 10.1080/23288604.2016.1222794[published Online First: Epub Date]|.

208. Boak G, Dickens V, Newson A, et al. Distributed leadership, team working and service improvement in healthcare. Leadership in Health Services 2015;28(4):332-44 doi: 10.1108/LHS-02-2015-0001[published Online First: Epub Date]|.

209. Best A, Berland A, Greenhalgh T, et al. Networks as systems: A case study of the World Health Organization's Global Health Workforce Alliance. Journal of Health, Organisation and Management 2018;32(1):9-24 
210. World Health Organization. The World Health Report 2000, Health Systems: Improving performance. Geneva: World Health Organization, 2000.

211. Alliance for Health Research Policy and System Research (AHPSR). Open mindsets: Participatory leadership for health. Geneva: World Health Organization, 2016.

212. Best A, Saul J. Complexity and lessons learned from the Health Sector for Country System Strengthening: Background paper for the USAID experience summit on strengthening country systems. US Agency for International Development (USAID), November 2012.

213. Lowe T, French M, Hawkins M, et al. New development: Responding to complexity in public services-the human learning systems approach. Public Money \& Management 2020:1-4 doi: 10.1080/09540962.2020.1832738[published Online First: Epub Date]|.

214. Ministry of Health. Healthy Familes NZ. 11 January 2021. https://www.health.govt.nz/ourwork/preventative-health-wellness/healthy-families-nz.

215. Te Puni Kōkiri. About Whānau Ora. 6 October 2020. https://www.tpk.govt.nz/en/whakamahia/whanau-ora.

216. Willis $C D$, Best $A$, Riley $B$, et al. Systems thinking for transformational change in health. Evidence \& Policy: A Journal of Research, Debate and Practice 2014;10(1):113-26 doi: 10.1332/174426413x662815[published Online First: Epub Date]|.

217. Sheridan N, Kenealy T, Schmidt-Busby J, et al. Population health in New Zealand 20002013: From determinants of health to targets. SAGE open medicine 2015;3:2050312115573654-54 doi: 10.1177/2050312115573654[published Online First: Epub Date]|.

218. Steele Gray C, Berta W, Deber R, et al. Organizational responses to accountability requirements: Do we get what we expect? Health care management review 2017;42(1):65-75 doi: 10.1097/HMR.0000000000000089[published Online First: Epub Date]|.

219. Victoria Health and Human Services. Victorian Health Services Performance Monitoring Framework 2018-19. https://www2.health.vic.gov.au/hospitals-and-healthservices/funding-performance-accountability/performance-monitoring.

220. New Zealand Government. Record investments in hospitals and health services. 2020. https://www.beehive.govt.nz/release/record-investment-hospitals-and-health-services. 221. Johnston M. Budget 2020: No joy for primary care. New Zealand Doctor 202014 May. 222. Maude S. Budget 2020: Māori health leader's scathing response. New Zealand Doctor 2020 14 May. 
223. Maude S. Budget 2020: Business advisor rues ill-tempered budget bodes badly for general practice. New Zealand Doctor 202015 May.

224. Cassie F. Budget 2020: Lost opportunity to reshape primary care - Robin Gauld. New Zealand Doctor 202015 May.

225. Middleton L, Dowdle D, Villa L, et al. Saving 20000 Days and Beyond: A realist evaluation of two quality improvement campaigns to manage hospital demand in a New Zealand District Health Board. BMJ Open Quality 2019;8(4) doi: http://dx.doi.org/10.1136/bmjoq-2018-000374[published Online First: Epub Date]|.

226. New Zealand Labour Party. Our manifesto to keep New Zealand moving: Labour 2020. 2020. https://drive.google.com/file/d/13uhcVrn8HUXEoWoPQgkJYjHX_d_Za-O0/view. 